Maintenance of Borrelia burgdorferi s.I. diversity in enzootic cycles

Elena Claudia Coipan 


\section{Thesis committee}

\section{Promotors}

Prof. Dr W. Takken

Personal chair at the Laboratory of Entomology

Wageningen University

Prof. Dr H.H.T. Prins

Professor of Resource Ecology

Wageningen University

\section{Co-promotor}

Dr H. Sprong

Research coordinator wildlife and vector-borne diseases

National Institute for Public Health and the Environment, Bilthoven

\section{Other members}

Prof. Dr B.J. Zwaan, Wageningen University

Prof. Dr R.A. Coutinho, Utrecht University

Dr D. Heylen, University of Antwerp, Belgium

Prof. Dr M.E. Visser, Wageningen University

This research was conducted under the auspices of the C. T. de Wit Graduate School for Production Ecology and Resource Conservation 


\title{
Maintenance of Borrelia burgdorferi s.I. diversity in enzootic cycles
}

\author{
Elena Claudia Coipan
}

Thesis

submitted in fulfilment of the requirements for the degree of doctor at Wageningen University

by the authority of the Rector Magnificus

Prof. Dr A.P.J. Mol,

in the presence of the

Thesis Committee appointed by the Academic Board

to be defended in public

on Wednesday 9 November 2016

at 4 p.m. in the Aula. 
Elena Claudia Coipan

Maintenance of Borrelia burgdorferi s.I. diversity in enzootic cycles B5, 180 pages.

PhD thesis, Wageningen University, Wageningen, NL (2016)

With references, with summary in English

ISBN 978-94-6257-957-6

DOI http://dx.doi.org/10.18174/392288 


\section{Contents}

Chapter1 General introduction 1

Chapter 2 Few vertebrate species dominate the Borrelia burgdorferi 9 s.l. life cycle

Chapter 3 Geodemographic analysis of Borrelia burgdorferi sensu lato using the 5S-23S rDNA spacer region

Chapter 4 Contribution of rodent species to maintenance of tickborne pathogens in enzootic cycles

Chapter 5 Spatiotemporal dynamics of emerging pathogens in 77 questing Ixodes ricinus

Chapter 6 Imbalanced presence of Borrelia burgdorferi s.I.

multilocus sequence types in clinical manifestations of Lyme borreliosis

Chapter 7 General discussion

References

Summary

Acknowledgements

Curriculum vitae

Publications

PE\&RC PhD Education Certificate 



\section{Chapter 1}

\section{General Introduction}

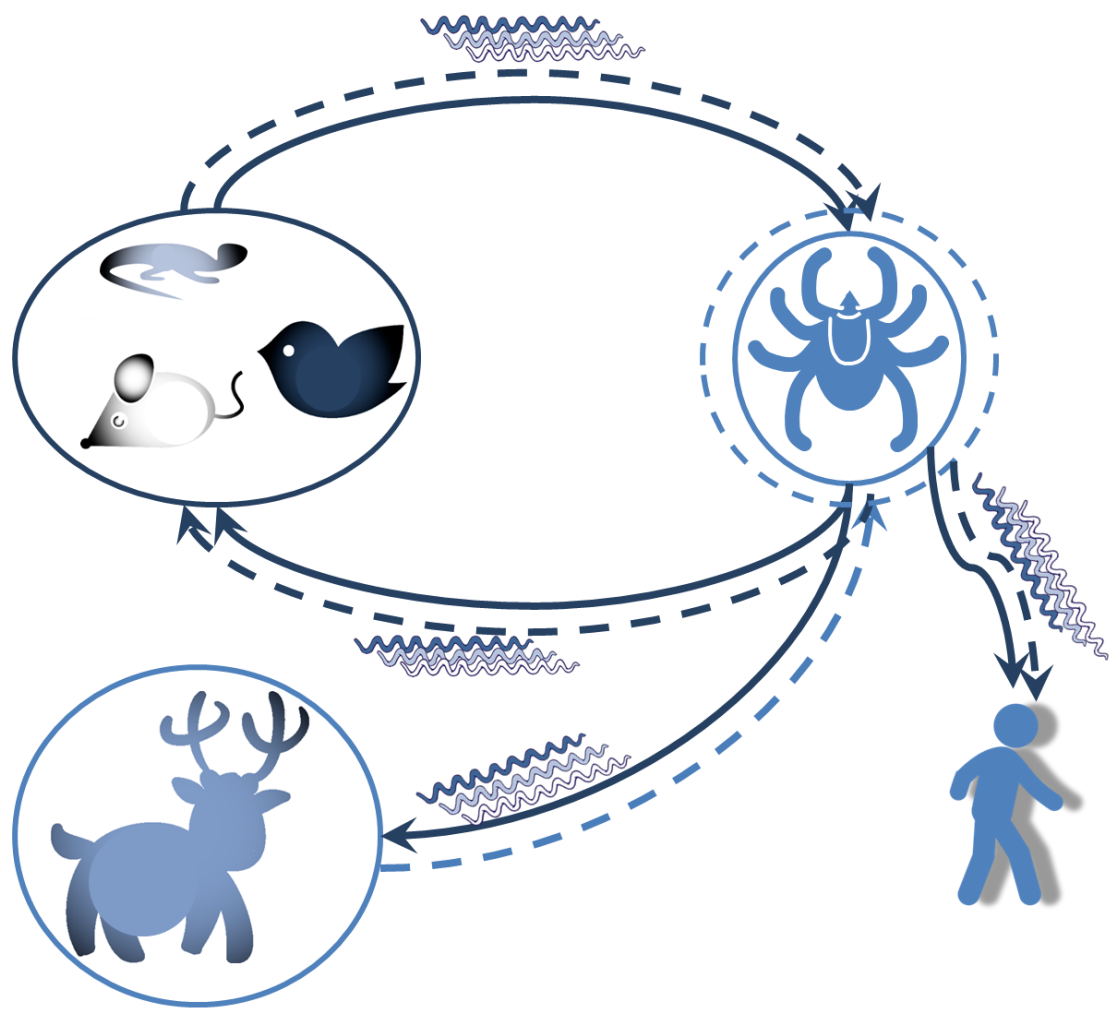

Schematic representation of the maintenance of $B$. burgdorferi s.l. in enzootic cycles. The various genospecies and genotypes of bacteria are transmitted from the competent vertebrate hosts to the ticks feeding on them and from here back to other competent or non-competent Borrelia hosts as well as, occasionally, to humans (continuous dark-blue arrows). The transmission cycle of the bacteria is interrupted when they reach a non-competent Borrelia host or humans. However, the non-competent hosts can act still as feeding hosts for the tick (dotted light-blue arrow), leading to an augmentation of the tick population (dotted blue circle). This in turns will lead to an augmentation in the abundance of the infected ticks and therefore an augmented Borrelia transmission (dotted dark-blue arrows) with subsequent increased exposure of humans. 


\section{Chapter 1}

Lyme borreliosis is probably the most prevalent tick-borne disease in humans. Its causative agents, Lyme spirochaetes from the Borrelia burgdorferi sensu lato complex are transmitted by Ixodes ricinus ticks in Europe. In several European countries, including The Netherlands, the incidence of Lyme borreliosis has been on the rise for the last decades. In order to define effective intervention strategies for controlling the disease, more insight in the transmission dynamics of tick-borne pathogens, both between animals and ticks, but also from ticks to humans is needed. A Dutch research program called "Shooting the messenger" has focussed on several key aspects that together determine the acarological risk.

The research in this PhD thesis focussed on linking the transmission cycles of Lyme spirochaetes to the different clinical manifestations of Lyme borreliosis.

\section{Maintenance of Borrelia burgdorferi s.l. diversity in enzootic cycles}

Notwithstanding the causality dilemma of the "egg and the hen" as to whichever transmitted the first Borrelia burgdorferi s.l. spirochaete - the host or the vector, it is assumed that the enzootic cycle of these spirochaetes begins with competent vertebrate hosts. These can carry, amplify, and transmit the bacteria to the blood-sucking vectors that feed on them i.e. ticks. The ticks that manage to maintain the Borrelia spirochaetes through the moulting process can transmit them further to a next vertebrate they feed on... and the transmission cycle of $B$. burgdorferi s.l. resumes. A Borrelia transmission cycle that has been shown to involve up to 18 different Ixodes and more than 300 vertebrate species. When accounting for densities of some individual host species of up to 1200 and ticks of up to two million per square kilometre, the simple transmission cycle becomes a process of enormous proportions. When you consider also that one of the feeding hosts of the ticks is represented by humans, the simple transmission cycle becomes a complicated public health issue, with an incidence of more than 65,000 of Lyme borreliosis cases in Europe alone (Hubalek 2009)!

Borrelia burgdorferi s.l. is a group of 20 genospecies of spirochaetes, some of which are known as Lyme disease spirochaetes. The disease was named from the Lyme District in Connecticut, USA, where it was first diagnosed (Burgdorfer et al. 1982). Later, the causative bacteria were identified as highly motile spirochaetes that are transmitted by hard ticks (Acari: Ixodida)(Burgdorfer et al. 1983). During the last three decades Lyme disease has gained increasingly more interest, being identified as the most common vector-borne disease in the temperate area of the northern hemisphere (ECDC 2011). With an intricate enzootic cycle and a genetic complexity to match it, B. burgdorferi s.l. is one of the most puzzling pathogenic microorganisms. This thesis addresses the ecology 
and molecular adaptations of $B$. burgdorferi s.l. at various scales, from complex to genospecies level, pinpointing the implications for public health.

\section{Transmission cycle}

Borrelia burgdorferi s.l. is a vector-borne microorganism - it cannot be transmitted between vertebrate hosts in the absence of a tick vector. Ixodes ricinus Linnaeus is the main tick vector of $B$. burgdorferi s.I. in Europe (Gern and Humair 2002b).

The transovarial transmission is considered to have a negligible contribution to the maintenance of the bacteria in enzootic cycles (Richter et al. 2012,Rollend et al. 2013). However, recently, van Duijvendijk et al. (2016) have shown that $0.62 \%$ of the larvae in nature is infected with $B$. burgdorferi s.l. The presence of the spirochaetes in the larvae could be the result of partial feeding of larvae on a host with a subsequent change of host, but it could also be the result of transovarial transmission. Future studies have to clarify the importance of this transmission route in the maintenance of $B$. burgdorferi s.l. and the transmission to humans.

The main transmission route of these bacteria is the interstadial one, from larvae to nymphs and from nymphs to adult ticks. Larvae of $I$. ricinus can become infected during a blood meal from an infected host (Piesman and Sinsky 1988) and during a blood meal in the vicinity of an infected nymph feeding on a host, a process known as co-feeding (Gern and Rais 1996). The infected engorged larvae then moult into infected nymphs, which can transmit the spirochaetes to new hosts (Radolf et al. 2012). The same process is repeated for the next developmental stage - nymph to adult. Thus, the maintenance of the bacteria in enzootic cycles is dependent on various species of vertebrates and the ticks that feed on them. Deer are among the few vertebrates known as incompetent for transmission of B. burgdorferi s.l. (Pacilly et al. 2014). The inability of Borrelia to circumvent the innate immune response of ungulates, make these animals incompetent transmitters of the spirochaetes (Kurtenbach et al. 2002a).

Although there have been several studies carried out on particular sites (e.g. (Matuschka et al. 1991)) or on various classes of vertebrates (e.g. (Matuschka et al. 1990)), and some descriptive reviews have been published (e.g. (Mannelli et al. 2012), (Franke et al. 2013),(Pfaffle et al. 2013)), there is no quantitative review that integrates data on a host assemblage comprising a wide range of vertebrate species. 
How do the vertebrate hosts contribute to the maintenance of $B$. burgdorferi s.l.?

Maintenance of the bacteria in enzootic cycles occurs via direct transmission between various vertebrate hosts and hard ticks (Acari: Ixodida). Tick and host associations shape, thus, the geographical distribution of $B$. burgdorferi s.l. (Kurtenbach et al. 2006b,Vollmer et al. 2011). Most of the Borrelia burgdorferi s.l. genospecies are specialist in terms of the class of vertebrate hosts that they exploit. In general, they are either mammal-, avian- or reptile-associated.

At large geographical scale the distribution of the various Borrelia genospecies is primarily driven by the vertebrate host they are adapted to (Kurtenbach et al. 2006b,Vollmer et al. 2011), with bird-associated Borrelia having a wider areal than rodent-associated ones. Thus, bird-associated Borrelia, such as B. garinii, B. turdi, and B. valaisiana, are spread over both Europe and Asia. The genospecies that are mammal-associated, such as $B$. spielmanii, $B$. yangtze, and $B$. tanukii, seem to be confined to certain geographic areas (Fukunaga et al. 1996,Margos et al. 2015,Margos et al. 2011,Richter et al. 2004). Exceptions are $B$. afzelii and $B$. bavariensis, which are spread across all Eurasia (Margos et al. 2013, Rauter and Hartung 2005).

Host species differ in their transmission capacity of different genospecies or genotypes of $B$. burgdorferi s.l. to I. ricinus ticks. Both the number of ticks a host can feed and the transmission of $B$. burgdorferi s.l. could be linked to general host characteristics (Carbone et al. 2005, Lee 2006,Previtali et al. 2012), and could therefore influence both tick burden and reservoir competence for $B$. burgdorferi s.l. (Barbour et al. 2015, Huang et al. 2013, Marsot et al. 2012). The infection dynamics, the success of transmission and maintenance of $B$. burgdorferi s.l. in enzootic cycles depends mostly on the density and abundance of the various vertebrate host species. Therefore, investigations on the vertebrate species composition and their abundance in host communities in various habitats will allow for better predictions of the bacterial genotypes circulating in those particular habitats.

Genetic differentiation is a precondition for speciation (Avise 2007). The host community has been hypothesised to generate the intraspecific genetic diversity of $B$. burgdorferi s.l. by various mechanisms: the multiple niche polymorphism balancing selection that implies that various hosts can act as ecological niche for a subset of the strains of a species (Gliddon and Strobeck 1975 ,Levene 1953) and the negative frequency dependent polymorphism. This latter mechanism postulates that no strain has a maximum fit within a certain 
host species but that initial infection of a host triggers an immune response that will be protective against subsequent infections with genetically similar bacterial strains (Barthold 1999,Gromko 1977).

The maintenance mechanisms of $B$. burgdorferi s.I. in enzootic cycles are relevant when trying to design intervention and control strategies for Lyme borreliosis. Using the $B$. afzelii-rodent system as an example, should all rodents have equal importance in maintaining $B$. afzelii, a dilution effect can be attempted only using non-rodent mammalian species. If, by contrast, the various rodent species are responsible for maintenance of different $B$. afzelii genotypes, then, based on the severity of the symptoms that those genotypes incur in humans, the control strategies could be targeted towards the rodent species responsible for the transmission of the most aggressive bacterial genotypes.

Coinfection with other microorganisms may facilitate or impair the transmission efficiency of the Borrelia. Multiple studies have reported coinfection in questing ticks with some of the tick-borne pathogens (TBPs) other than B. burgdorferi spp. (Belongia 2002,Burri et al. 2011,Ginsberg 2008,Lommano et al. 2012,Nieto and Foley 2009, Reye et al. 2010). It is possible that the severity of Lyme disease is affected by simultaneous infections with other TBPs (Belongia 2002,Swanson et al. 2006). Some of them, such as $A$. phagocytophilum, modulate host immunity and increase susceptibility to various secondary pathogens, including B. burgdorferi s.l. (Holden et al. 2005, Thomas et al. 2001). Coinfection might be, therefore, partly responsible for the transmission efficiency of $B$. burgdorferi s.l. between hosts and ticks, but also for the variability in clinical manifestations of Lyme borreliosis. One of the valid questions is, thus, which are the microorganisms that are commonly associated with $B$. burgdorferi s.l.?

\section{How do ticks contribute to the maintenance of B. burgdorferi s.I.?}

Some $B$. burgdorferi s.l. genospecies are vectored by different tick species in different geographical areas. e.g. bird-associated Borrelia have a cycle that involves I. frontalis, I. turdus, and I. columnae in Asia (Masuzawa et al. 1996a,Miyamoto and Masuzawa 2002) and I. ricinus and I. persulcatus in Europe (Gern and Humair 2002b). Likewise, B. burgdorferi s.s. is transmitted in Europe by $I$. ricinus and I. hexagonus (Gern and Humair 2002b,Toutoungi and Gern 1993) and in North America by I. scapularis and I. pacificus (Piesman 2002).

While some Ixodes species transmit multiple $B$. burgdorferi s.l. genospecies, other tick-Borrelia associations seem to be less efficient (Masuzawa et al. 2005). It is possible that the bacterium-tick interaction contributes to the augmentation of the host spectrum of the bacterium. The tick species present in 


\section{Chapter 1}

the areal of a Borrelia burgdorferi s.l. genospecies could promote genetic differentiation of the bacteria and differential transmission efficiencies by various mechanisms.

One of these mechanisms relies on the intrinsic properties of the ticks such as the receptors for the spirochaetal proteins. Some of the proteins important for the persistence of the bacteria in ticks are OspA and OspB, their removal leading to the failure of the spirochaetes to colonize the tick midgut ( $\mathrm{Pal}$ et al. 2000,Pal et al. 2004). Different receptors for OspA (Pal et al. 2004) could account for different attachment rates of the spirochaetes to the tick midgut and, hence, for their abundance in enzootic cycles. Such a situation could be that of $B$. burgdorferi s.s. in North America. While it is relatively rare in the questing $I$. ricinus ticks - less than 2\% (Coipan et al. 2013c,Rauter and Hartung 2005), it is much more frequent in I. scapularis and I. pacificus - 25-35\% (Kurtenbach et al. 2006a). Furthermore, while in Europe, this is a bacterium that infects only mammals, especially rodents of the Sciuridae family (Humair and Gern 1998, Marsot et al. 2011,Pisanu et al. 2014), in the Nearctic it is the dominant Borrelia burgdorferi s.l. genospecies, thriving in a variety of vertebrate hosts of all classes (mammal, avian, and reptilian) (Piesman 2002).

Both the genetic diversity of $B$. burgdorferi s.l. and their abundance in enzootic cycles are influenced by the host range of the tick species; whether a tick is a generalist or a specialist will implicitly affect the circulation of the bacteria it carries in enzootic cycles. It could be that the specialization of $B$. burgdorferi s.l. genospecies is partly influenced by the tick feeding behaviour. Evolutionary theory predicts that specialist pathogens are favoured if their hosts are abundant, whereas generalists would do better when the encounters with host species are less predictable (Woolhouse et al. 2001). The feeding pattern of tick stages could, thus, explain the prevalence of the various B. burgdorferi s.l. in questing ticks.

\section{Which are the implications for public health?}

The most frequently Borrelia genospecies retrieved from human cases of Lyme borreliosis are B. afzelii, B. garinii, B. burgdorferi s.s., and B. bavariensis (Stanek et al. 2012). The genetic differences between the genospecies seem to translate not only in different enzootic associations but also in differential pathogenicity for humans (Stanek et al. 2012). It is known that B. afzelii is mostly associated with erythema migrans (EM) and acrodermatitis chronica atrophicans (ACA) (Stanek et al. 2012) while B. garinii infections can lead to neurological symptoms - the so-called neuroborreliosis (NB). However, each of these genospecies have multiple genotypes, as defined by multilocus sequence typing 
(MLST) (Margos et al. 2008,Urwin and Maiden 2003). Whether each of these genotypes within the pathogenic Borrelia genospecies present in questing ticks can cause disease in humans or there is only a subset of them that is pathogenic (infectious), is of relevance for public health. Research on B. burgdorferi s.s. in North America has shown that the genetic makeup of the pathogenic spirochaetes is a determinant for the symptomatology they cause (Dykhuizen et al. 2008, Hanincova et al. 2013,Strle et al. 2011,Wormser et al. 2008). It seems, thus, that discriminating between these types may be useful for disease risk assessment and management, but knowledge on the infectiousness and subsequent clinical symptoms of European Borrelia genospecies is, however, scarce.

Currently, the acarological risk of human infection with B. burgdorferi s.I. is defined as the density of infected questing nymphs (Dister et al. 1997, Glass et al. 1994,Glass et al. 1995,Kitron and Kazmierczak 1997,Nicholson and Mather 1996), as the nymphs are considered as constituting the main source of human infection with Borrelia burgdorferi s.l. Thus, all Borrelia genospecies are considered equally hazardous for humans. The study of pathogenicity of the various Borrelia genospecies and genotypes should allow for individual hazard assignment. The combination of hazard and exposure (prevalence in questing ticks) would then allow individual genospecies/genotypes risk assessment.

Thus, both ecological and clinical studies are necessary to be able to address the public health issue that is nowadays collectively called Lyme borreliosis.

\section{Thesis aim and outline}

The aim of this thesis was to link the ecology of B. burgdorferi s.l. with its pathogenicity for humans. To this end, I addressed the topic of genetic diversity of Borrelia burgdorferi s.l., how this is maintained in enzootic cycles, and how it impacts on public health.

The objectives of the thesis were:

- $\quad$ identification of the main vertebrate hosts for the various B. burgdorferi s.l. genospecies

- $\quad$ linking the composition of B. burgdorferi s.l. in questing ticks with that in human clinical manifestations

In order to identify the main vertebrate hosts responsible for the maintenance of $B$. burgdorferi in enzootic cycles, but also for feeding Ixodes ricinus ticks, we conducted a meta-analysis on literature data. The analysis 


\section{Chapter 1}

described in chapter 2 identifies small rodents and thrushes as main vertebrate hosts and discusses implications for the transmission of the spirochaetes.

In chapter 3 we prove that the 5S-23S rDNA intergenic spacer (IGS) is a suitable molecular marker for identification of $B$. burgdorferi s.l. to genospecies level, but also to characterize the genetic diversity of the spirochaetes at intragenospecies level and to detect genetic differentiation between the subpopulations of Borrelia. Consequently, we used this marker repeatedly in combination with other genetic markers, in the studies addressing the genetic diversity of Borrelia in small mammals and humans (see following Chapters).

Chapter 4 focuses on the rodents, which were identified in the literature meta-analysis as being the main hosts for I. ricinus larvae as well as for Borrelia afzelii. We tested the multiple niche polymorphisms hypothesis, using IGS, dbpA and ospC as molecular markers for typing $B$. afzelii genotypes in fed larvae collected from rodents in various areas in The Netherlands. Additionally, we compared the quantitative role of bank voles and wood mice in B. afzelii and Neoehrlichia mikurensis maintenance, another emerging tick-borne pathogen in Europe.

The density of the vertebrate hosts and the feeding preferences of the ticks should logically determine the prevalence of $B$. burgdorferi s.l. genospecies in questing ticks. We address this topic in Chapter 5 , while screening also for the presence of other tick-borne pathogens. To test whether these pathogens might share similar enzootic cycles we looked for patterns of coinfection and seasonal dynamics of infection in questing. . ricinus nymphs.

Chapter 6 addresses the pathogenicity of $B$. burgdorferi s.l. genospecies and genotypes for humans, using IGS and MLST as molecular markers. The frequency of the Borrelia in humans is compared to the frequency in questing ticks to assess the infectivity of the various genospecies and genotypes. In order to assess the invasiveness of the bacterial genospecies and/or genotypes we compared their frequency in the late/disseminated (acrodermatitis chronica atrophicans and neuroborreliosis) and the incipient (erythema migrans) forms of Lyme borreliosis.

The findings of Chapters 2-6 are discussed in Chapter 7, in the light of current knowledge in the field and underlining potential future avenues for research. 


\section{Chapter 2}

\section{Few vertebrate species dominate the}

\section{Borrelia burgdorferi s.I. life cycle}

This chapter has been published as:

Hofmeester, T.R., Coipan, E.C., van Wieren, S.E., Prins, H.H.T., Takken, W., Sprong, H., 2016. Few vertebrate species dominate the Borrelia burgdorferi s.l. life cycle. Environmental Research Letters 11, 43001-43016. 


\title{
Chapter 2
}

\begin{abstract}
Background. In the northern hemisphere, ticks of the Ixodidae family are vectors of diseases such as Lyme borreliosis, Rocky Mountain spotted fever and tick-borne encephalitis. Most of these ticks are generalists and have a three-host life cycle for which they are dependent on three different hosts for their blood meal. Finding out which host species contribute most in maintaining ticks and the pathogens they transmit, is imperative in understanding the drivers behind the dynamics of a disease.

Methods. We performed a systematic review to identify the most important vertebrate host species for Ixodes ricinus and Borrelia burgdorferi s.l. as a well-studied model system for tick-borne diseases. We analyzed data from 66 publications and quantified the relative contribution for 15 host species.

Review results. We found a positive correlation between host body mass and tick burdens for the different stages of I. ricinus. We show that nymphal burdens of host species are positively correlated with infection prevalence with B. burgdorferi s.l., which is again positively correlated with the realized reservoir competence of a host species for B. burgdorferi s.l. Our quantification method suggests that only a few host species, which are amongst the most widespread species in the environment (rodents, thrushes and deer), feed the majority of $I$. ricinus individuals and that rodents infect the majority of $I$. ricinus larvae with $B$. burgdorferi s.l.
\end{abstract}

Discussion. We argue that small mammal-transmitted Borrelia spp. are maintained due to the high density of their reservoir hosts, while birdtransmitted Borrelia spp. are maintained due to the high infection prevalence of their reservoir hosts. Our findings suggest that Ixodes ricinus and Borrelia burgdorferi s.l. populations are maintained by a few widespread host species. The increase in distribution and abundance of these species, could be the cause for the increase in Lyme borreliosis incidence in Europe in recent decades. 


\section{Background}

Zoonotic vector-borne diseases pose an increasing threat to human health, as one-third of the emerging infectious diseases in the last decades was vector-borne (Jones et al. 2008). In the northern hemisphere, ticks of the Ixodidae family are vectors for human diseases such as Lyme borreliosis, Rocky Mountain spotted fever and tick-borne encephalitis (Jongejan and Uilenberg 2004). From these, the spirochaete complex Borrelia burgdorferi sensu lato ((Baranton et al. 1992); from here on referred to as B. burgdorferi), the causative agent of Lyme borreliosis and vectored by ticks of the Ixodes ricinus complex ( $\mathrm{Xu}$ et al. 2003), causes the majority of human disease cases (Dantas-Torres et al. 2012). Both in Europe and in North America, I. ricinus and I. scapularis populations have spread and increased in density in recent decades, most probably due to a multitude of man-made changes to the environment, which has resulted in an increase in Lyme disease incidence (Kurtenbach et al. 2006a,Medlock et al. 2013).

Lyme disease risk is determined by multiple biological, environmental and societal factors (Mannelli et al. 2012,Randolph 2004,Vanwambeke et al. 2010). These can be split into two distinct groups, (1) factors determining the number of questing Ixodes ticks infected with $B$. burgdorferi, and (2) factors determining the level of human exposure to ticks (Sprong et al. 2012a). In this review, we will focus on the first, with in particular the factors that determine the number of questing Ixodes ticks, and their infection with B. burgdorferi.

Both I. ricinus and $B$. burgdorferi are considered generalist parasites, as they utilize a multitude of host species (Jaenson et al. 1994,Margos et al. 2011). These host species differ considerably in the numbers of ticks they feed, which differs between the different life stages of the tick (Gray 1998,Talleklint and Jaenson 1994). Ixodes ricinus has three life stages, larva, nymph and adult, which need a blood meal from a vertebrate host during each stage to moult to the next stage or to lay eggs (Gray 1998). Host species differ in their ability to infect $I$. ricinus larvae with different genospecies of $B$. burgdorferi. For example, B. afzelii is mainly transmitted by small mammals, while $B$. garinii is mainly transmitted by birds (Hanincova et al. 2003a, Hanincova et al. 2003b), and even within genospecies, different host species differ in their ability to transmit $B$. burgdorferi (Kurtenbach et al. 1994). Both the number of ticks a host can feed and the transmission of $B$. burgdorferi could be linked to general host characteristics, such as host body mass, which is related to both immunological and behavioral responses (Carbone et al. 2005, Lee 2006,Previtali et al. 2012), and could therefore influence both tick burden and reservoir competence for $B$. burgdorferi (Barbour et al. 2015, Huang et al. 2013,Marsot et al. 2012). 


\section{Chapter 2}

The success of transmission and maintenance of $B$. burgdorferi in enzootic cycles depends, therefore, on the density and abundance of the various vertebrate host species. As the transmission of $B$. burgdorferi from one host to another is mediated by ticks, the distribution of the various genospecies is also dependent on the behavior and feeding preference of the vector ticks. Thus, the resulting $B$. burgdorferi distribution in the questing ticks is a function of the densities of different host species, their capacity to feed ticks and their capacity to transmit the bacteria to those ticks. Therefore, it is important to summarize data on the distribution of ticks of different stages over different vertebrate host species and use these data to find patterns that could be related to the increase in disease risk due to indirect effects by human induced changes to the environment.

Although there have been several studies carried out on particular sites (e.g. (Matuschka et al. 1991) or on various classes of vertebrates (e.g. (Matuschka et al. 1990), and some descriptive reviews have been published (e.g. (Franke et al. 2013,Mannelli et al. 2012,Pfaffle et al. 2013), there is no quantitative review that integrates data on a host assemblage comprising a wide range of vertebrate species. Here, we used a data driven approach to quantify the contribution of various vertebrate host species to feeding $I$. ricinus ticks, and transmitting $B$. burgdorferi to feeding larvae, to infer a mechanism that could support the apparent increase in Lyme borreliosis incidence in Europe. Furthermore, pinpointing the host species groups that are most important in feeding $I$. ricinus might aid in selecting host species to target intervention strategies (Perkins et al. 2003).

We compiled data on interactions between vertebrate hosts, I. ricinus and B. burgdorferi using a systematic review approach. For the species for which data were available that fulfilled our selection criteria, we looked for correlations between: (1) body mass and I. ricinus burden for the different stages, (2) nymphal burden and infection prevalence with $B$. burgdorferi, and (3) infection prevalence and realized reservoir competence for $B$. burgdorferi. We hypothesize that host species body mass is positively correlated to I. ricinus burden, as host species of greater body mass have a greater day range (Carbone et al. 2005) and are therefore more likely to encounter ticks in the vegetation. Furthermore, we hypothesize that the I. ricinus burden of a host species is positively correlated with the infection prevalence with $B$. burgdorferi as hosts that feed a large number of ticks are more likely to feed an infected tick and become infected. Lastly, we hypothesize that the average infection prevalence of a host species is positively correlated with the realized reservoir competence of a host species, as hosts that are more often infected are more likely to transmit the disease to a large number of larvae. 
Next to these general patterns, we aimed to quantify the relative contribution of different host species in the maintenance of $I$. ricinus and $B$. burgdorferi. For this, we modified the framework proposed by Mather et al. (1989) to quantify the importance of different vertebrate species based on differences in density, I. ricinus burden and realized reservoir competence for $B$. burgdorferi. The original framework (Mather et al. 1989) was created to quantify the relative importance of different host species in infecting I. scapularis larvae with $B$. burgdorferi in three study sites in North America. We modified the equations to quantify the relative importance of host species in the feeding of $I$. ricinus as well as the relative importance in infecting larvae with $B$. burgdorferi. As these equations need a vertebrate assemblage for their calculation, we used data from the literature search to create an assemblage including the most widespread vertebrate species occurring in most European forests.

\section{Methods}

We performed a literature search using PubMed, Web of Science and Scopus to review the parasitism of Ixodes ricinus on vertebrate host species, and the occurrence of Borrelia burgdorferi in vertebrate hosts and in the I. ricinus parasitizing them. We only considered European host species. Our most extensive search was done in PubMed, were we searched for publications in English and German. The last literature search was carried out in January 2015 and concerned the years 1945-2014. The search string used, and part of the selection procedure are given in the supplementary material. An additional screening of relevance concentrated on the type of data the publications included: field-derived or laboratory data. As we used the data for a quantification framework resembling a situation in the field, we chose only publications that contained field-derived data. Finally, we selected for papers including data on: (1) measurements of the tick burden on the vertebrate hosts, (2) measurements of host infection prevalence with B. burgdorferi, or (3) measurements of infection prevalence with $B$. burgdorferi in feeding ticks. Publications with incomplete or previously published data were excluded. All publications were reviewed by two different contributors (TRH and ECC) and the data extracted from each paper were checked twice.

\subsection{Data acquisition}

For each of the selected publications the following variables were extracted: location, number of hosts examined, number of hosts infested, number of hosts infected, numbers of $I$. ricinus of each stage per host, method of $B$. burgdorferi detection, type of tissue tested, number of samples (ticks or tissue) tested, number of samples (ticks or tissue) positive and genospecies of $B$. 


\section{Chapter 2}

burgdorferi detected. These variables were the primary variables in our database, and were used for subsequent calculations. Difficulties in extracting data resulted from reported data that accounted for total number of ticks only (no stage mentioned) or combined observations of multiple host species. These papers were stored in the database, but not used for further analysis. To fill the database with values for the desired analyses, the following steps were carried out. If the number of infested or infected hosts or ticks in the study was not given, it was calculated, if possible, based on the number of samples examined and the reported infestation or infection prevalence. Similarly, if the number of ticks infesting the host animals was not given, then we would calculate it from the number of animals examined and their mean infestation. Most of the time, however, a total number of ticks from a specific stage collected from a total number of hosts was given, so we could calculate the average tick burden per stage. Only about one third of the publications (56/162) contained standard deviations, standard errors or confidence intervals for the parameters we were interested in, so we decided to calculate merely an average value and no other descriptive statistical parameters.

Publications were divided into separate records if the investigators examined (1) different host species, (2) hosts collected in different locations (if specified), (3) different tick stages (4) different Borrelia genospecies, or (5) hosts tested also by xenodiagnosis. From publications in which the same ticks were examined with different methods for B. burgdorferi detection, leading to different results, only data obtained by PCR or sequencing were included. Records containing sequencing data were considered to have tested for the presence of all the $B$. burgdorferi genospecies described in the paper. Combining these records resulted in a database on tick burdens, host infection prevalence, and infection prevalence in feeding ticks per host species with data from 162 publications (supplementary data).

\subsection{Summarizing the data}

As most studies presented only total numbers of animals and ticks studied, we calculated the average tick burden for each stage of tick for each host species by using equation (1)

$$
B_{l_{i}}=\frac{\sum_{s=1}^{n} L_{i_{S}}}{\sum_{s=1}^{n} H_{i_{s}}}
$$

Where, $B_{l_{i}}$ is the mean per-capita larval burden of species $i, L_{i_{s}}$ is the total number of larvae counted on host species $i$ in study $s$, and $H_{i_{s}}$ is the total number of individuals of host species $i$ studied in study $s . L_{i_{s}}$ can be substituted 
by the total number of nymphs counted on species $i$ in study $s\left(N_{i_{s}}\right)$ or by the total number of adults counted on species $i$ in study $s\left(A_{i_{s}}\right)$ in order to calculate the mean per-capita nymphal burden $\left(B_{n_{i}}\right)$ or the mean per-capita adult burden $\left(B_{a_{i}}\right)$, respectively. Our term tick burden is equal to the mean density of ticks as defined by Kahl et al (2002).

Infection with $B$. burgdorferi was calculated as the sum of infections with the various genospecies and with untyped $B$. burgdorferi, counting the mixed infections only once. We only considered $B$. burgdorferi and not the individual genospecies to be able to use data produced before widespread use of molecular techniques, and to facilitate comparison between different host taxa.

We calculated two different measures of infection. For each host species we calculated the infection prevalence $\left(\mathrm{IP}_{\mathrm{bb}_{\mathrm{i}}}\right)$ of that species with $B$. burgdorferi by using equation (2). Our term infection prevalence is equal to the ratio of pathogen-exposed hosts versus non-exposed hosts (Kahl et al. 2002) and indicates the fraction of animals within a species that has been infected by $B$. burgdorferi

$$
I P_{b b_{i}}=\frac{\sum_{s=1}^{n} B B I H_{i_{S}}}{\sum_{s=1}^{n} H_{i_{S}}}
$$

$I P_{b b_{i}}$ is the infection prevalence of species $i$ with any genospecies of $B$. burgdorferi, $B B I H_{i_{s}}$ is the total number of $B$. burgdorferi infected host individuals of species $i$ in study $s$ and $H_{i_{s}}$ is the total number of host individuals of species $i$ sampled in study $s$. Infection can be determined by either testing tissue samples from a host, or by testing $I$. ricinus for presence of $B$. burgdorferi after they were collected from a host in the field. As these can, potentially, result in very different values, both were calculated separately and were named 'tissue-derived' infection prevalence and 'tick-derived' infection prevalence, respectively.

Next to that, we calculated the realized reservoir competence $\left(\mathrm{RC}_{\mathrm{bb}}\right)$, i.e., the proportion of blood fed larvae that become infected with $B$. burgdorferi (LoGiudice et al. 2003), by using equation (3), which is comparable to the specific host infectivity as defined by Kahl et al (2002)

$$
R C_{b b_{i}}=\frac{\sum_{s=1}^{n} B B I L_{i_{S}}}{\sum_{s=1}^{n} L_{i_{S}}}
$$

$R C_{b b_{i}}$ is the realized reservoir competence of species $i$ for any genospecies of $B$. burgdorferi, $B B I L_{i_{s}}$ is the total number of $B$. burgdorferi 
infected larvae sampled from host species $i$ in study $s$, and $L_{i_{S}}$ is the total number of larvae tested from species $i$ in study $s$.

\subsection{Selection criteria}

In order to improve the data quality in our analysis, we selected data from our database using the following criteria: (1) a minimum of 20 individuals from a species at a location was studied, (2) a minimum of 50 larvae was tested to determine the realized reservoir competence, (3) the study was conducted within the activity period of I. ricinus, for which we excluded studies performed in December-February, and studies that were performed all year round without specifying numbers for the separate seasons and (4) the study was conducted within habitats in which I. ricinus normally resides, namely forest, forest ecotone and woodland. Lastly, we excluded studies that only considered migratory birds. This resulted in a dataset with data for 44 species from 66 publications.

\subsection{Quantifying the role of species as hosts for $I$. ricinus and $B$. burgdorferi}

We used this dataset to quantify the role of fifteen species as hosts for $I$. ricinus and $B$. burgdorferi, using modifications of the framework proposed by Mather et al (1989). The original formula was used to quantify the relative importance of host species in infecting larval I. scapularis with B. burgdorferi. We modified the original equation to be used to calculate the relative importance of host species in feeding the different stages of I. ricinus (equation (4)), as well as the relative importance of host species in infecting $I$. ricinus larvae with $B$. burgdorferi (equation (5)). For equation (5), we separated the number of infected vectors produced by the host species ( $N_{S}$ in the original model) into two different parameters, $B_{l_{i}}$ and $R C_{b b_{i}}$ We did this to clarify the similarity between the two equations we used.

$$
\begin{aligned}
& R I_{l_{i}}=\frac{B_{l_{i}} D_{i}}{\sum_{j=1}^{n} B_{l_{j}} D_{j}} \\
& R I_{b b_{i}}=\frac{B_{l_{i}} D_{i} R C_{b b_{i}}}{\sum_{j=1}^{n} B_{l_{j}} D_{j} R C_{b b_{j}}}
\end{aligned}
$$

$R I_{l_{i}}$ is the relative importance of host species $i$ in feeding the larval stage of I. ricinus, $B_{l_{i}}$ is the mean per-capita larval burden of species $i$, and $D_{i}$ is the density $\left(\mathrm{km}^{-2}\right)$ in which species $i$ occurs. The $\sum_{j=1}^{n} B_{l_{j}} D_{j}$ gives the total number of larvae fed by all species in the assemblage, as determined by their mean larval burden and their densities. $B_{l_{i}}$ can be substituted by $B_{n_{i}}$ or $B_{a_{i}}$ to calculate the 
relative importance of a host species in feeding the nymphal $\left(R I_{n_{i}}\right)$ and adult $\left(R I_{a_{i}}\right)$ stages, respectively.

$R I_{b b_{i}}$ is the relative importance of species $i$ in infecting larvae with any genospecies of $B$. burgdorferi and is equivalent to the relative reservoir capacity used by Kahl et al (2002) or the reservoir potential of Mather et al (1989). This parameter could also be seen as the relative contribution of a host species to the pool of infected nymphs. We did not calculate the relative importance of host species for the separate $B$. burgdorferi genospecies, because there were not enough data available for multiple host species in our selection.

As the quantification of the relative importance of a species is dependent on all species in an assemblage, we needed to select a number of host species to perform our calculations. In principle the equations can be used for any specific area where local densities and tick burdens are known. To present the idea behind the framework, and to show some overall trends that we think might be true for any area, we selected a hypothetical assemblage of species. We chose a relatively diverse European forest vertebrate assemblage consisting of six mammalian and nine avian species (table 1). All of these species occur regularly in north-western European forests or forest ecotones. Species were selected based on their area of distribution throughout Europe as described in published handbooks (Cramp and Perrins 1994,Niethammer and Krapp 1978) and on the number of individuals (minimum of 100 individuals) that was studied in the publications used for data acquisition. Densities of the species were collected from the same published handbooks used for determining the area of distribution. Although the relative importance is calculated per host species, we divided the host species into different species groups based on size and taxonomy (table 1). We present only these broad group contributions, to show general patterns regardless of the contribution of individual species.

To test for the sensitivity of the framework to errors in the mean percapita tick burden, we calculated the relative importance of the host groups for additional scenarios, in which the species composition of the host assemblage remained unaltered but in which the contribution of rodents, birds or ungulates varied by either doubling or halving the mean per-capita tick burden of these groups compared to the initial values, while all other parameters were kept constant.

\subsection{Statistical analysis}

To test for correlations between body mass, $I$. ricinus burden, $I P_{b b_{i}}$ and $R C_{b b_{i}}$ of the different host species, we performed a stepwise analysis. First we 


\section{Chapter 2}

tested for correlations between body mass and I. ricinus burden, secondly, we tested for correlations between I. ricinus burden and $I P_{b b_{i}}$ and thirdly, we tested for correlations between $I P_{b b_{i}}$ and $R C_{b b_{i}}$. For the species that were considered in our vertebrate assemblage for the quantification of the importance of different host species, we also tested for correlations between density and body mass. Statistical analyses were performed using R 3.2.2 (R_Core_Team 2015). All analyses were performed for each host taxa (birds, mammals and reptiles) separately.

Table 1. Host species considered in our model assemblage, their taxonomic group and the density that was used in the calculations.

\begin{tabular}{llr}
\hline Scientific name & Host taxonomic group & Density $\left(\mathrm{km}^{-2}\right)$ \\
\hline Apodemus sylvaticus & Rodent & 1200 \\
Capreolus capreolus & Ungulate & 11 \\
Cyanistes caeruleus & Small bird & 200 \\
Erinaceus europaeus & Medium-sized mammal & 1 \\
Erithacus rubecula & Small bird & 80 \\
Fringilla coelebs & Small bird & 100 \\
Microtus agrestis & Rodent & 1000 \\
Myodes glareolus & Rodent & 1200 \\
Parus major & Small bird & 100 \\
Phylloscopus collybita & Small bird & 100 \\
Prunella modularis & Small bird & 200 \\
Sylvia atricapilla & Small bird & 40 \\
Turdus merula & Thrush & 200 \\
Turdus philomelos & Thrush & 80 \\
Vulpes vulpes & Medium-sized mammal & 1 \\
\hline
\end{tabular}

We used log-log regressions to test for correlations between host body mass and $I$. ricinus burden for the three life stages using average body mass of the host species obtained from published handbooks (Cramp and Perrins 1994,Niethammer and Krapp 1978). This was done because the average tick burdens per life stage did not yield integers, which refrained us from performing generalized linear models using Poisson or negative binomial distributions. Because of the presence of zeros, we added the lowest non-zero burden to the tick burdens ( 0.04 for larvae, and 0.01 for nymphs and adults) in order to calculate the $\log _{10}$. Due to the large variation in body size, we also $\log _{10}$ transformed host body mass. To give more weight to species that were studied 
more intensively, we weighted the log-log regression by sample size. We also used log-log regressions for testing the correlations between density and body mass of host species.

For both the infection prevalence and the realized reservoir competence we used a generalized linear model with a binomial distribution and a logit link. For the infection prevalence we used the number of host individuals found infected and the number of host individuals found uninfected, using the tissuederived data, to test for a correlation between infection prevalence and $\log _{10}$ nymphal burden. We supplemented this dataset with tick-derived data for species for which tissue-derived data were missing. For the realized reservoir competence we used, per host species, the total number of larvae found infected and the total number of larvae found uninfected as reported in the selected papers, to test for a correlation between realized reservoir competence and logit infection prevalence. By using the binomial infected-non-infected data, we weighted the correlations by sample size. We tested if the models for realized reservoir competence could be improved by adding $\log _{10}$ body mass to the model, and compared the models using AICc values (Burnham and Anderson 2004) using the package MuMIn (Barton 2014). To further analyze the impact of species averages taken from few studies with low sample sizes, we did a posthoc analysis of leverage to check for the importance of single species in determining the regression coefficients. We calculated Cook's distance for all parameters in all analyses (Cook 1977). If a Cook's distance was larger than 0.5, we checked the number of studies and the number of individuals on which the estimate was based.

\section{Review results}

\subsection{Tick burdens, infection prevalence and realized reservoir competence of hosts}

The 44 host species in our dataset differed ten to thousandfold in I. ricinus burden, infection prevalence with $B$. burgdorferi and realized reservoir competence for $B$. burgdorferi (table 2). Because we only had data on three reptile species, we performed analyses on mammals and birds only. Larval $I$. ricinus burden increased with host body mass for birds $\left(F_{1,18}=12.1, p=0.02\right.$, $B=0.97)$ but not for mammals $\left(F_{1,15}=0.9, p=0.37\right)$. Nymphal $I$. ricinus burden was positively correlated to host body mass both for birds $\left(F_{1,18}=30.5, p<0.001\right.$, $B=1.81$; figure $1(A))$ and for mammals $\left(F_{1,15}=26.1, p<0.001, b=0.79\right.$; figure $1(D))$. Adult $I$. ricinus burden also increased with host body mass both for birds $\left(F_{1,18}=74.4, \quad p<0.001, \quad B=0.53\right)$ and for mammals $\left(F_{1,15}=73.9, p<0.001\right.$, $B=1.15)$. 


\section{Chapter 2}

The 25 host species for which we had data on infection prevalence with $B$. burgdorferi differed tenfold in infection prevalence (table 2). Infection prevalence increased with nymphal $I$. ricinus burden both for birds (deviance difference $=199.1, p<0.001, b=1.76$; figure $1(B)$ ) and for mammals (deviance difference $=24.6, p<0.001, B=0.34$; figure $1(E)$ ). Of the 17 host species for which we had data on the realized reservoir competence for $B$. burgdorferi, 14 also had data on infection prevalence (table 2). In these species, realized reservoir competence for $B$. burgdorferi increased with infection prevalence both for birds (deviance difference $=1048.2, p<0.001, b=1.29$ figure $1(\mathrm{C})$ ) and for mammals (deviance difference $=903.7, p<0.001, b=0.72$; figure $1(F)$ ). For both groups, the model improved significantly by adding $\log _{10}$ body mass, which was positively correlated to realized reservoir competence in birds $(\triangle \mathrm{AICC}=59.7$, $\left.p<0.001, B_{\mid \mathrm{P}}=0.54, B_{\text {body mass }}=2.88\right)$, and negatively correlated to realized reservoir competence in mammals $\left(\triangle \mathrm{AICC}=149.6, p<0.001, b_{\mathrm{IP}}=1.15, b_{\text {body }}\right.$ mass $=-3.13)$.

Post-hoc analyses of leverage indicated that for most analyses there were one or two species with a Cook's distance $>0.5$. However, most of the time these were the estimates which we gave a higher weight based on high sample size. In the few instances that species with a low sample size (less than 100 individuals) and low number of studies (less than three studies) had a high Cook's distance, omitting these species in the analysis only increased the fit. The only exception was the analysis of realized reservoir competence for mammals, for which omitting the data for Eliomys quercinus and Sorex araneus decreased the fit of the model including only infection prevalence with $B$. burgdorferi (deviance difference $=0.25, p=0.62$ ). However, excluding these two species from the model including both infection prevalence with $B$. burgdorferi and $\log _{10}$ body mass resulted in a similar result as for all species, albeit with slightly different parameter estimates (deviance difference $=96.91, p<0.001, \quad B_{\mathbb{P}}=0.76$, $\left.B_{\text {bodymass }}=-3.80\right)$.

\subsection{Relative importance of host groups for I. ricinus and B. burgdorferi}

The quantification of the relative importance of host species feeding $I$. ricinus indicated that rodents contributed most (89\%) to feeding larval I. ricinus (figure 2). Although the absolute value changes with different scenarios (range: $80 \%-94 \%$ ), rodents were the most important host group feeding I. ricinus larvae in all our scenarios (figure S1). Thrushes were the second most important group by feeding $5 \%$ of the larvae (range: $3 \%-9 \%$ ), followed by smaller birds $(4 \% ; 2 \%-$ $8 \%$ ). 

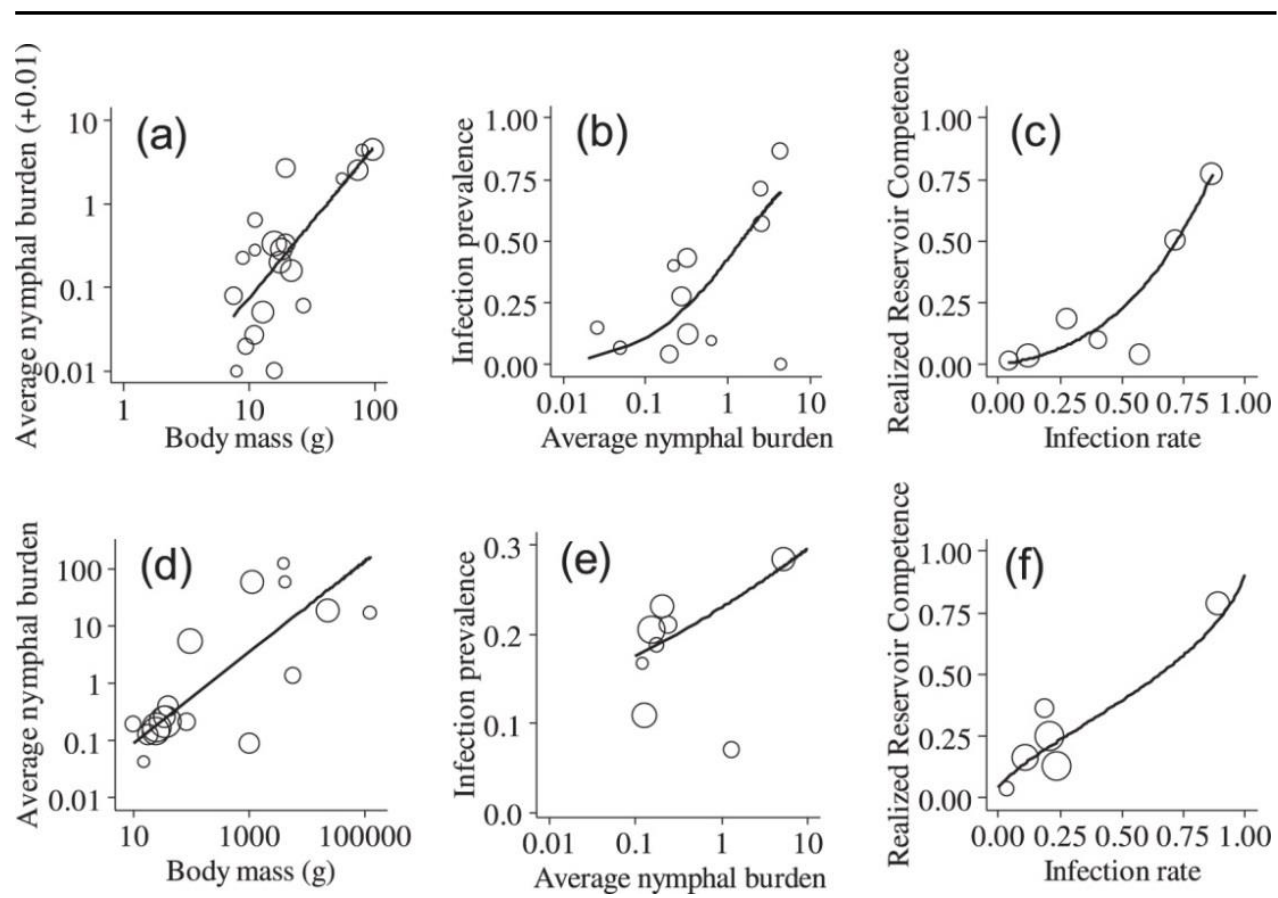

Figure 1. Correlations between nymphal $I$. ricinus burden, infection prevalence with $B$. burgdorferi and realized reservoir competence for $B$. burgdorferi. (A) Log-log regression between host species body mass and average nymphal $I$. ricinus burden for birds. (B) Binomial regression between average nymphal $I$. ricinus burden and infection prevalence with $B$. burgdorferi for birds. (C) Binomial regression between infection prevalence with $B$. burgdorferi and realized reservoir competence for $B$. burgdorferi for birds. (D) Log-log regression between host species body mass and average nymphal $I$. ricinus burden for mammals. (E) Binomial regression between average nymphal I. ricinus burden and infection prevalence with $B$. burgdorferi for mammals. (F) Binomial regression between infection prevalence with $B$. burgdorferi and realized reservoir competence for $B$. burgdorferi for mammals. For each regression the sample size used to determine the value on the $y$-axis is represented by the size of the circles in the plot.

The relative importance of host groups for nymphs differed most strongly between scenarios. Thrushes had the highest contribution to feeding nymphal $I$. ricinus (40\%; $29 \%-49 \%)$, while rodents (28\%; $16 \%-43 \%)$, small birds $(23 \%$; $16 \%-$ $28 \%)$ and ungulates $(8 \% ; 4 \%-14 \%)$ were also important, depending on the scenario. Ungulates contributed most (92\%) to feeding adult $I$. ricinus (figure 2). The absolute value of the importance of ungulates feeding adults changed per scenario (range: $85 \%-96 \%$ ), but in all scenarios the majority of adult $I$. ricinus is 
fed by ungulates. The second most important group were medium-sized mammals (5\%; 3\%-9\%).

The relative importance of host species for $B$. burgdorferi was calculated using only a subset of the host species in the dataset for which realized reservoir competence estimates were available (9 of the 15 species; table 2). In all scenarios, rodents were the most important host group infecting larval $I$. ricinus with B. burgdorferi (89\%; $80 \%-94 \%)$, followed by thrushes $(10 \% ; 5 \%-18 \%$ ). However, it has to be noted that no data were available on realized reservoir competence for any of the medium-sized mammals and ungulates. For the fifteen species in our calculations, density decreased with body mass for mammals $\left(F_{1,4}=10.05, p=0.03,6=-0.99\right)$ but not for birds $\left(F_{1,7}=0.12, p=0.74\right)$.

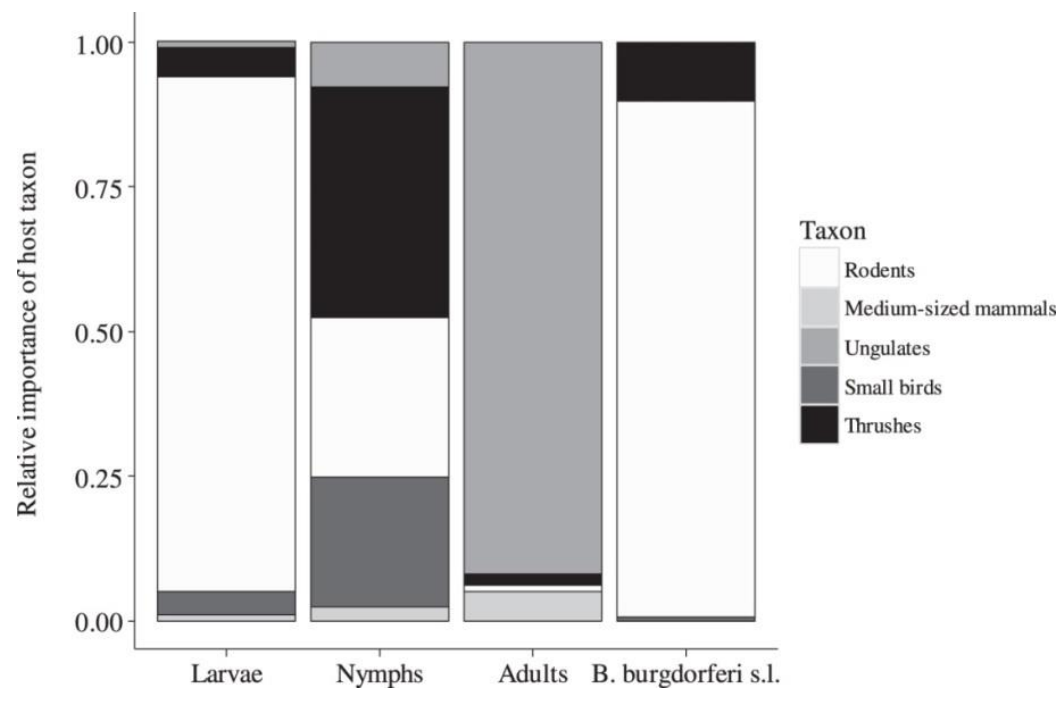

Figure 2. Quantification of the relative importance of different host groups feeding $I$. ricinus larvae, nymphs and adults, and infecting I. ricinus larvae with $B$. burgdorferi.

\section{Discussion}

\subsection{Importance of host species in maintaining I. ricinus}

Although I. ricinus is found to parasitize a large number of host species (Anderson and Magnarelli 1993), we found that the different stages of $I$. ricinus mainly feed on a few host species from different taxonomic groups. For birds, we found that species with higher body mass feed most $I$. ricinus of all stages. As host body mass is not correlated with host density in birds, it is the same large species of bird that contribute most to tick feeding by birds. In our analysis these 
were two thrushes, Turdus merula and Turdus philomelos, two abundant and widespread species in many European countries (Cramp and Perrins 1994). These species mostly forage on the ground, shifting through the litter layer looking for food (Cramp and Perrins 1994), which might be the main reason for the relatively high number of $I$. ricinus found on these species.

For mammals, which occur in the highest densities, we found a negative correlation between density and body mass, but no relationship between larval burden and body mass. Therefore, small mammals, occurring in high densities and having relatively large larval burdens, emerged from our analysis as the host group that was most important for feeding larval $I$. ricinus. This suggests that larval I. ricinus do not actively select for a host species, but rather feed on the hosts that are most abundant low in the vegetation where they quest (Mejlon and Jaenson 1997). The main studied small mammal host species were Apodemus flavicollis, Apodemus sylvaticus, Microtus agrestis, and Myodes glareolus, all widespread and abundant species in many European countries (Niethammer and Krapp 1978).

When looking at nymphs and adults, however, there was a strong correlation between host body mass of mammals and I. ricinus burden. This was reflected in our analysis by the higher relative importance of medium-sized mammals and ungulates for these stages, although small mammals were still the most important mammalian host group for nymphs, due to their high densities. Ungulates were the most important host group feeding adult I. ricinus, which suggests that adult $I$. ricinus actively select large mammals as hosts, regardless of their relatively low densities. This might be why adult ticks quest higher in the vegetation compared to nymphs and larvae (Mejlon and Jaenson 1997). In our analysis of host importance the only ungulate species included was Capreolus capreolus, the most widespread ungulate species in Europe (Niethammer and Krapp 1978). In the absence of roe deer, other ungulate species can serve as important hosts for adults as well.

Tick distributions on hosts are often highly over-dispersed (Randolph 2004) and summarizing this distribution by a mean value might not result in the best parameter. Nevertheless, most papers used in our analysis did not specify any other parameters, which resulted in our use of a mean per-capita tick burden per species. We do not think that this has greatly influenced our results. The differences in mean per-capita tick burden between species are much larger than differences between studies for the same species, and most values used in our analysis are based on large sample sizes. Furthermore, the different scenarios we used in our framework showed the same general patterns, showing that these patterns are not very sensitive to changes in mean tick burden (figure 


\section{Chapter 2}

S1). We do, however, strongly urge for a standardized reporting system for summarizing the numbers of ticks found on hosts, for which the reporting of the number of hosts, the prevalence of infestation, the median intensity of infestation, including confidence intervals, and the exponent $k$ of the negative binomial distribution could be used (Rozsa et al. 2000).

We show that all stages of $I$. ricinus can be maintained by only a few host species that are widespread throughout Europe. This, together with a large distribution in suitable habitat and climatic conditions, explains why I. ricinus has such a wide distribution, and why it occurs in high densities in many areas with a vertebrate assemblage existing of widespread species. It also supports the hypothesis that the increase in Lyme borreliosis may be due to an increase in $I$. ricinus distribution and abundance (Medlock et al. 2013,Sprong et al. 2012a), following increases in range and abundance of widespread host species such as C. capreolus, M. glareolus and T. merula (Apollonio et al. 2010,Gregory et al. 2007,van Strien et al. 2015).

\subsection{Infection prevalence of host species with B. burgdorferi}

We found that for small to medium-sized mammals and birds, the infection prevalence of host species with $B$. burgdorferi increased with their nymphal burden, with a stronger pattern for birds compared to mammals (figure 1). We did not have data on infection prevalence for the largest mammals in our analysis: $C$. capreolus and Cervus elaphus. Studies that were not incorporated in our selection, for reasons outlined above, show that roe deer have high levels of antibody in their blood, and low infection prevalence in tissues (Hulinska et al. 2002,Pato et al. 2013,Pichon et al. 2000). These findings support the hypothesis that $B$. burgdorferi is unable to circumvent host complement from deer (Kjelland et al. 2011,Pacilly et al. 2014), which could also explain the incapability of deer to transmit Borrelia spirochaetes (Kurtenbach et al. 2002a). This shows that the relationship between number of nymphs feeding on a species and infection prevalence might not be linear, signifying the need for more data on infection prevalence in ungulates, other large mammals, and large bird species.

We estimated the infection prevalence of host species for $B$. burgdorferi using tissue-derived data as this is the best method to determine infection prevalence of animals, as not all infected animals carry ticks that can be tested (Hanincova et al. 2003a). For our analysis, we complemented the dataset with tick-derived data only for species for which tissue-derived infection prevalence was not available. The difference between estimates of both methods within species are much smaller than the differences between species (table 2). Therefore, we conclude that using a combination of methods did not strongly 


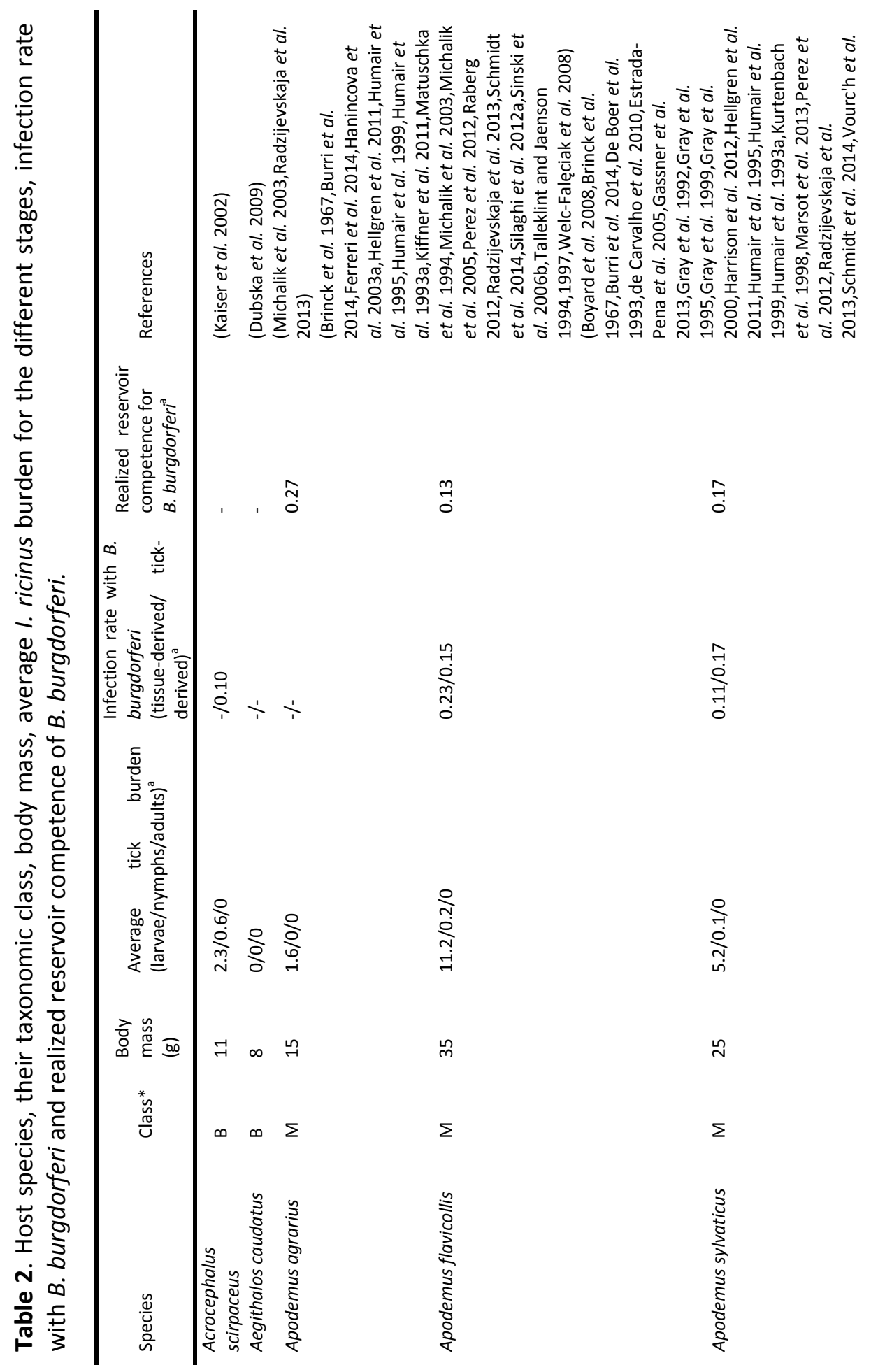




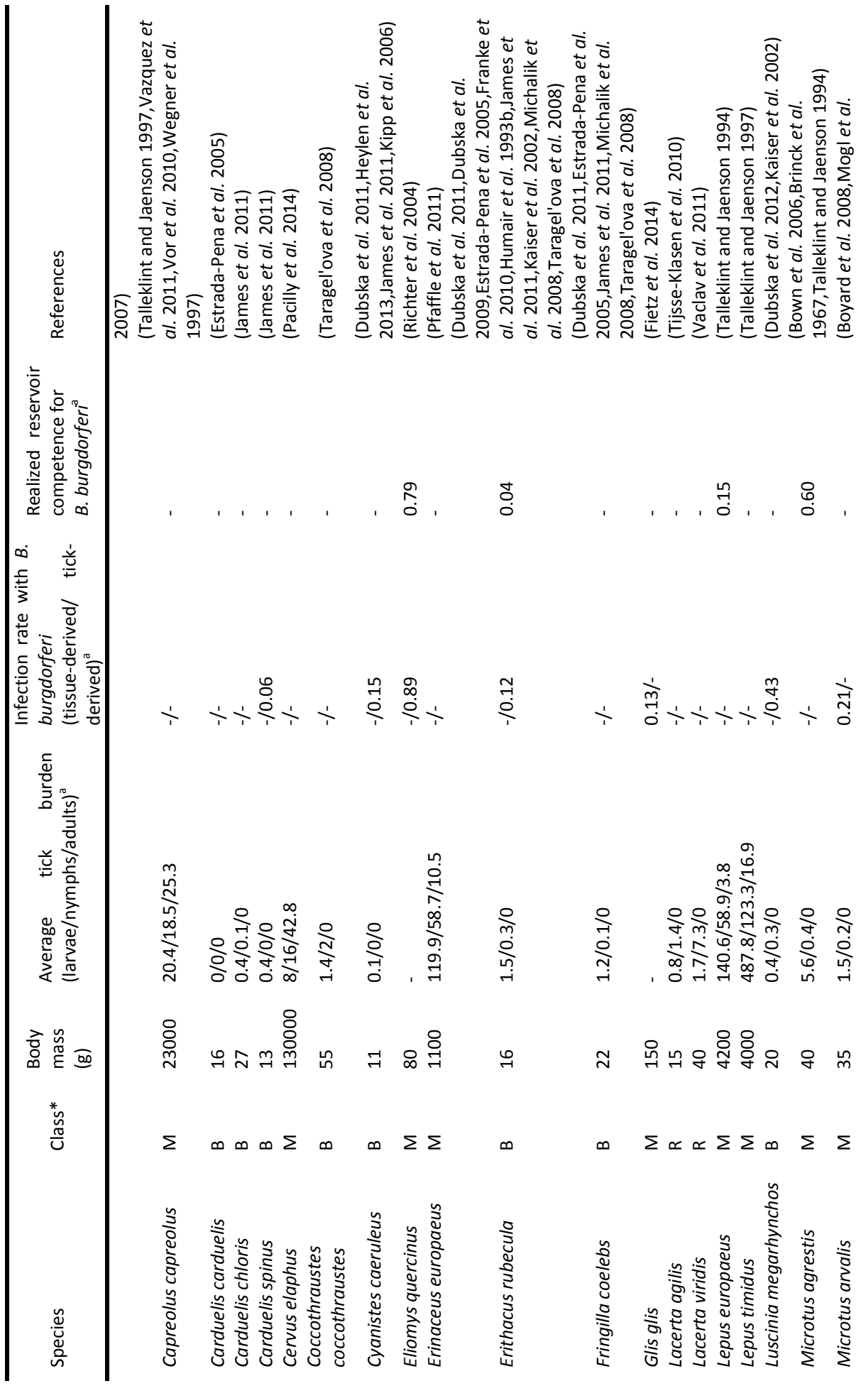




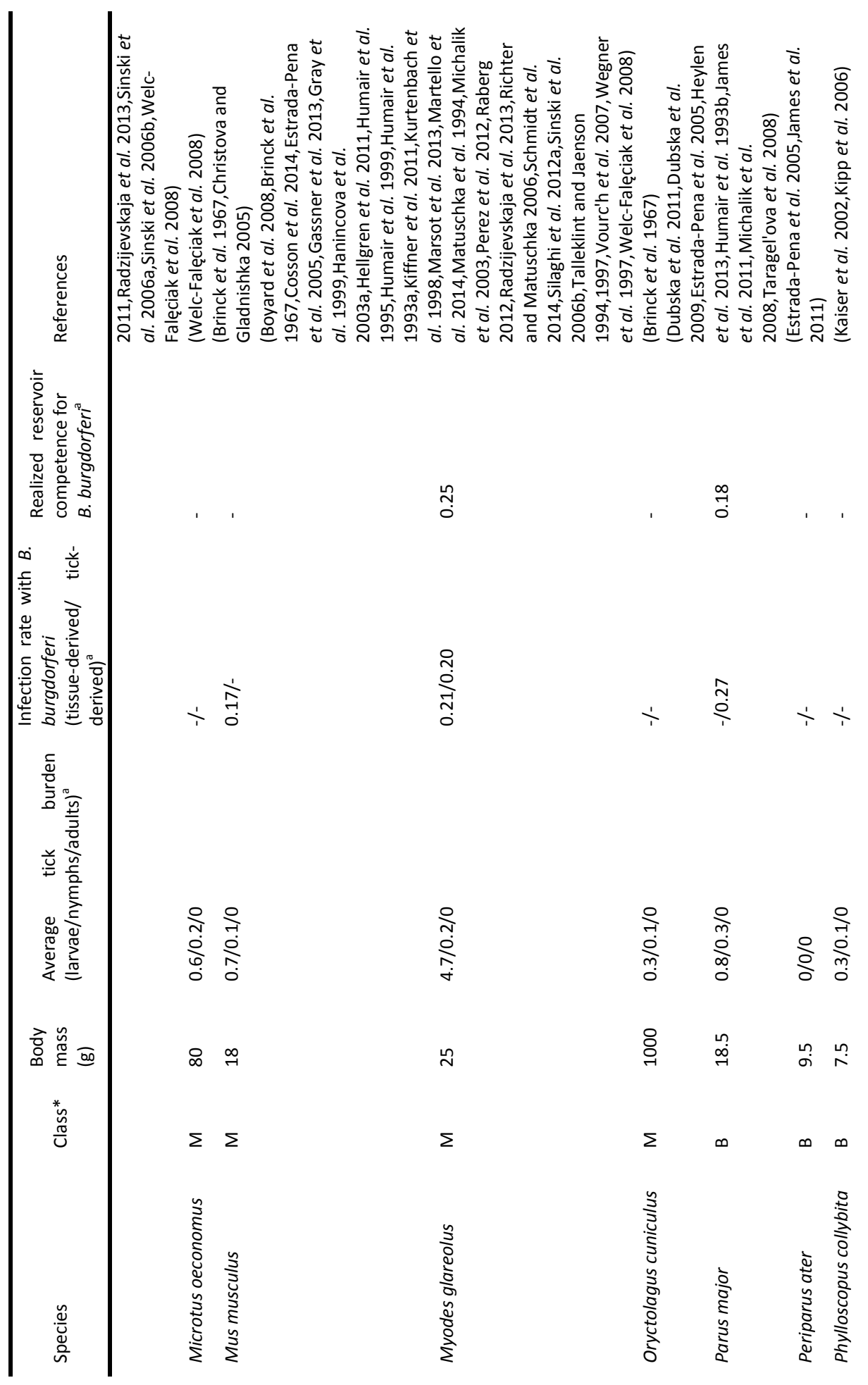




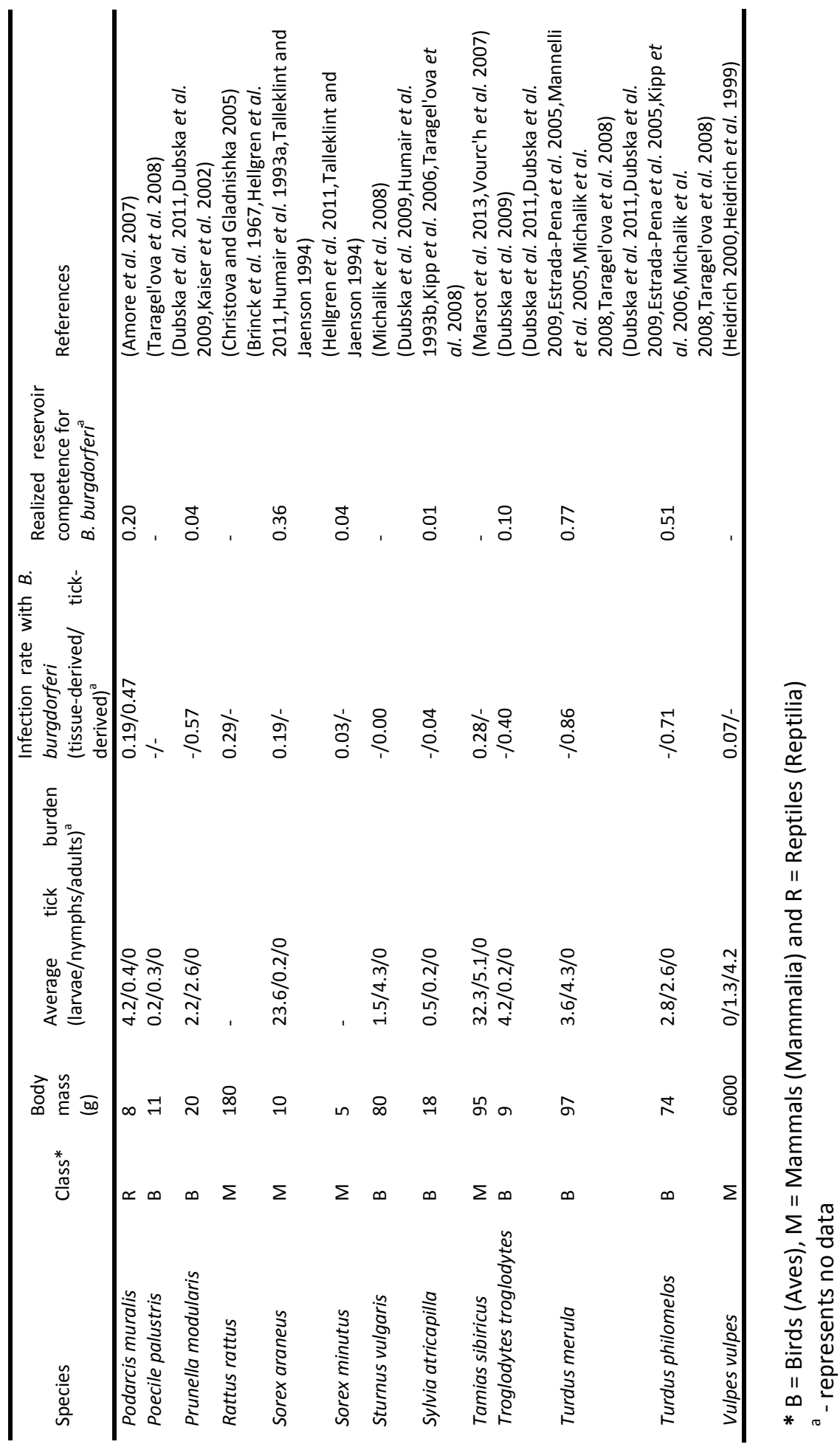


affect our results, although our results might be underestimates because not all infected animals carry infected ticks (Matuschka et al. 1993), and not all tissues from infected animals test positive (Kurtenbach et al. 1998).

We recommend future studies to test a combination of multiple tissues and engorged ticks to get the best possible estimate of infection prevalence of hosts with B. burgdorferi. For species that are able to transmit B. burgdorferi, xenodiagnosis using $I$. ricinus larvae will further increase the accuracy of infection prevalence estimates (De Boer et al. 1993).

\subsection{Realized reservoir competence for B. burgdorferi}

The realized reservoir competence for $B$. burgdorferi of mammals and birds $<100 \mathrm{~g}$ increases with $B$. burgdorferi infection prevalence of the species (figure 1). For small mammals we show a negative correlation between body mass and realized reservoir competence when we correct for differences in infection prevalence. It is hypothesized that smaller, short lived, species invest less in their immune system than larger, longer lived, species (Lee 2006). However, this hypothesis is debated for differences at a lower taxonomic level (Martin et al. 2007), so it might not be the most likely explanation for the correlation we found for rodents and shrews. We think it is more likely that $B$. burgdorferi has adapted to the species it most often encounters. This idea was supported by a positive correlation between density and realized reservoir competence of mammalian hosts for several tick-borne pathogens in North America (Ostfeld et al. 2014). We found a negative correlation between host body mass and density for mammals in our analysis, so the found correlation of realized reservoir competence with body mass could actually be the result of differences in densities of the different species.

For small songbirds we found a positive correlation between body mass and realized reservoir competence when we correct for differences in infection prevalence. This correlation could be caused by the two largest species (Turdus merula and $T$. philomelos) which have a far higher realized reservoir competence than the others. These species also have the highest nymphal burden and infection prevalence, showing that these are the bird species that $B$. burgdorferi most often encounters. Therefore, the adaptation of $B$. burgdorferi to the species it most often encounters could explain the different correlations with body mass we found for small mammals and birds. 


\subsection{Importance of host species in infecting larvae with B. burgdorferi}

In our analysis, rodents, which occur in high densities and have relatively large larval burdens, but relatively low realized reservoir competence, had the highest relative importance for infecting larvae with $B$. burgdorferi. Thrushes were the second most important group, having intermediate densities and larval burdens, but a very high realized reservoir competence. This indicates that the number of larvae feeding on a host species and its density are more important than the realized reservoir competence of that host species in determining the contribution of a host species to infect larvae. Furthermore, it suggests that the prevalence of different $B$. burgdorferi genospecies in questing ticks is mainly dependent on the distribution of larvae over rodents and thrushes.

The feeding pattern of ticks could explain why, in most areas in Europe, B. afzelii is the most common genospecies found in questing nymphs (Rauter and Hartung 2005). We found that $89 \%$ of the infected larvae in our analysis had fed on rodents. This should result in a large percentage of $B$. afzeliiinfected nymphs as $B$. afzelii is transmitted by small mammals (Hanincova et al. 2003a). Thrushes fed $10 \%$ of the infected larvae, which could explain the relatively low percentages of $B$. garinii and $B$. valaisiana in field-derived nymphs (Gassner et al. 2011).

\subsection{Borrelia spp. transmission maintenance}

The large difference in infection prevalence between small mammals and birds together with their large differences in relative importance for $B$. burgdorferi suggest that there are two distinct mechanisms behind the maintenance of small mammal-transmitted Borrelia spp. and bird-transmitted Borrelia spp. (Kurtenbach et al. 2002b). Because small mammals have low nymphal burdens, their infection prevalence with $B$. burgdorferi is relatively low (table 2). However, because they feed such a large proportion of the larvae, even a small infection prevalence of the host species can result in a high density of infected nymphs with small mammal-transmitted Borrelia spp. like $B$. afzelii. This high density of nymphs infected with small mammaltransmitted Borrelia spp. results in a sufficiently-large number of infected nymphs to, in turn, infect small mammals in spite of their low nymphal burdens.

Bird-transmitted Borrelia spp., like B. garinii and B. valaisiana, on the other hand, seem to be dependent on high infection prevalence of their host species due to relatively high nymphal and adult burdens (table 2). Therefore, 
even with a low larval burden and intermediate host density, sufficient numbers of infected nymphs are produced to infect birds, which completes the maintenance cycle for bird-transmitted Borrelia spp. However, this strategy is probably not only restricted to bird-transmitted Borrelia spp. Borrelia spielmanii is a candidate for a similar maintenance strategy in mammals as it is often found with low prevalence in questing ticks, but with high prevalence in one of its principal hosts, E. quercinus (Richter et al. 2004).

These differences in maintenance strategies could indicate that less common Borrelia spp., or other tick-borne pathogens with low infection prevalence in questing nymphs, might be maintained by host species with high nymphal or adult burdens (Ostfeld et al. 2014). Also it shows that $B$. burgdorferi can specialize either on host species that occur in high densities, or on host species that feed large numbers of ticks, with the exception of larger bodied mammalian species such as deer.

\subsection{Host species diversity}

Ostfeld and Keesing (2000) proposed a dilution effect of host species diversity on Lyme borreliosis risk. This hypothesis has been highly debated, especially in the context of ticks from the I. ricinus complex and Lyme borreliosis (Randolph and Dobson 2012,Wood and Lafferty 2013). Although our analysis did not examine the effect of differences in species richness, our methods could be used to quantify the relative contributions of different species in different assemblages, as long as differences in tick burden and density are accounted for. Our results suggest that few, but widespread vertebrate species feed most of the ticks in European forests. Therefore, community related factors influencing either the densities or tick burdens of these species can have an effect on the outcomes of the calculations. For example, the presence of predators could have effects on the densities or tick burdens of rodents, which may affect the number of $B$. burgdorferi infected ticks in the vegetation (Ostfeld and Holt 2004).

\subsection{Limitations of the data}

There is little information available on the infection prevalence with and realized reservoir competence for the different genospecies of $B$. burgdorferi such as B. afzelii, B. bavariensis, B. burgdorferi s.s., B. garinii, B. lusitaniae, $B$. spielmanii and $B$. valaisiana (supplementary data). For some widespread host species such as European hedgehog (Erinaceus europaeus), European hare (Lepus europaeus), and field vole (Microtus agrestis) there were no data available at the genospecies level that would satisfy our selection criteria. 
Furthermore, for some widespread host species, such as Eurasian badger (Meles meles), Eurasian jay (Garrulus glandarius), Eurasian pygmy shrew (Sorex minutus), Eurasian red squirrel (Sciurus vulgaris), European pine marten (Martes martes), great spotted woodpecker (Dendrocopos major), wild boar (Sus scrofa) and wood pigeon (Columba palumbus), either very little, or no data at all were available (supplementary data). For example, the two studies (Humair and Gern 1998,Pisanu et al. 2014) that lead the current opinion that red squirrels are important hosts in transmitting and maintaining $B$. burgdorferi s.s. in Europe had either a very low sample size (Humair and Gern 1998), or the animals were collected throughout the year in different habitat types, without specifying infection prevalence per season/habitat type (Pisanu et al. 2014). Therefore, we stress that data on tick stages and genospeciesspecific infection should be collected from these host species during the active period of $I$. ricinus, in natural habitats, in order to be able to analyse a more complete host assemblage. This would also enable the analysis of the relationships between host body mass, density, tick burdens, infection prevalence and realized reservoir competence for the different genospecies.

\section{Conclusion}

Our analysis suggests that a few vertebrate species that are widespread in Europe are the most important host species feeding $I$. ricinus and transmitting $B$. burgdorferi. We demonstrate that vertebrate species with a higher body mass have a higher I. ricinus burden, that host species with higher tick burdens are more likely to be infected with $B$. burgdorferi and that species that are more often infected with $B$. burgdorferi also transmit the infection more often to larval I. ricinus. These patterns suggest that $B$. burgdorferi adapts to the species it most often encounters.

To our knowledge, this review is the first to quantify the relative importance of host species for the different stages of I. ricinus, and our calculations support the widely held idea that small rodents are the most important hosts in feeding larval I. ricinus, that birds and rodents feed most of the nymphs, and that ungulates are the main hosts for adult I. ricinus e.g. (Gray 1998,Mannelli et al. 2012). We found that rodents and thrushes contribute most to the pool of $B$. burgdorferi infected nymphs. We suggest two different maintenance strategies for $B$. burgdorferi, which are correlated to high host densities or high infection prevalence of the hosts. These might explain how some tick-borne pathogens can be maintained with very low prevalence in questing ticks. We show that using a simple framework and a systematic data search can be used to calculate the relative importance of host species for tick 
species, and tick-borne pathogens, which can be used in research on other tick species and other tick-borne pathogens. These results can aid selection of host species to target for intervention strategies (Perkins et al. 2003).

Rodents, thrushes and deer, that are the most important host groups feeding $I$. ricinus and infecting $I$. ricinus larvae with $B$. burgdorferi, have increased in distribution and abundance in recent decades due to changes in land use and forest management (Apollonio et al. 2010,Gregory et al. 2007,van Strien et al. 2015), which could be the main driver behind increased disease incidence with tick-borne diseases in Europe. 
A)

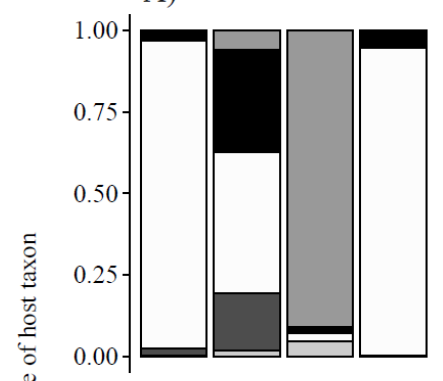

D)

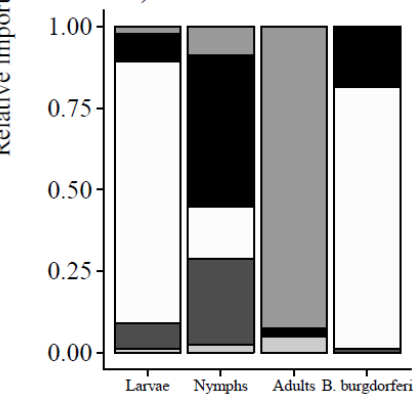

B)

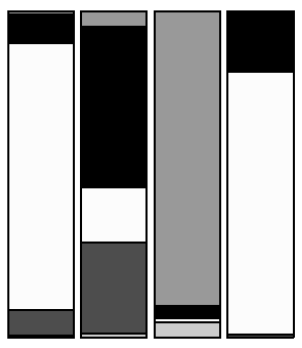

E)

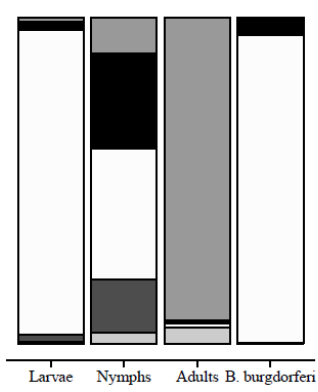

C)

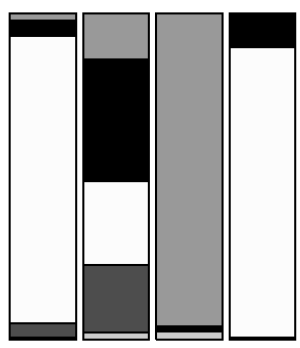

F)
Taxon

Rodents

Medium-sized mammals

Ungulates

Small birds

Thrushes

Figure S1. Different scenarios for the quantification of the relative importance of different host groups feeding $I$. ricinus larvae, nymphs and adults, and infecting $I$. ricinus larvae with $B$. burgdorferi. A) Twofold increase in rodent percapita tick burden for all stages. B) Twofold increase in bird per-capita tick burden for all stages. C) Twofold increase in ungulate per-capita tick burden for all stages. D) Twofold decrease in rodent per-capita tick burden for all stages. E) Twofold decrease in bird per-capita tick burden for all stages. F) Twofold decrease in ungulate per-capita tick burden for all stages. In each model, all other parameters were held constant. 


\section{Supplementary Material: Search string and part of the selection procedure}

The search string used was:

(Ixodes ricinus OR Borrelia burgdorferi) AND (distribution $O R$ presence $O R$ occurrence $O R$ reported $O R$ incidence $O R$ prevalence $O R$ spread $O R$ dispersion $O R$ detection $O R$ diagnosis $O R$ isolation $O R$ counts $O R$ burden $O R$ infestation) AND (Accipiter OR Acrocephalus OR Alauda OR Alca OR Alces OR Allactaga OR Alopex $O R$ Anas $O R$ Anser $O R$ Anthus $O R$ Apodemus $O R$ Aquila OR Arvicola $O R$ Asio OR Athene OR Bison OR Bomyicilla OR Bonasa OR Bos OR Bubalus OR Bubo OR Buteo OR Canis OR Capra OR Capreolus OR Caprimulgus OR Carduelis OR Castor OR Cepphus OR Certhia OR Cervus OR Charadrius OR Chionomys OR Chroicocephalus OR Clethrionomys OR Coccothraustes OR Columba OR Corvus OR Coturnix OR Crex OR Cricetulus OR Cricetus OR Crocidura OR Cuculus $O R$ Dama OR Darevskia OR Dendrocopos OR Dryomys OR Eliomys OR Emberiza OR Equus $O R$ Erinaceus OR Erithacus OR Eutamias OR Falco OR Felis OR Ficedula OR Francolinus OR Fringilla OR Fulica OR Gallinago OR Garrulus OR Genetta OR Glis $O R$ Hemiechinus $O R$ Hemorrhois $O R$ Hippolais $O R$ Hirundo $O R$ Jaculus $O R$ Jynx OR Lacerta OR Lagopus OR Lagurus OR Lanius OR Larus OR Lemmus OR Lepus $O R$ Locustella OR Loxia OR Luscinia OR Martes $O R$ Meles $O R$ Mesocricetus OR Micromys OR Microtus OR Miliaria OR Milvus OR Motacilla OR Mus OR Muscardinus OR Muscicapa OR Mustela OR Myocastor OR Myodes OR Neomys OR Netta OR Numenius OR Nyctereutes OR Ochotona OR Oenanthe OR Ondatra OR Oryctolagus OR Ovis OR Parus OR Passer OR Perdix OR Pernis OR Phalacrocorax OR Phasianus $O R$ Philomachus OR Phoenicurus $O R$ Phylloscopus OR Pica OR Picoides OR Picus OR Pluvialis OR Podarcis OR Podiceps OR Porzana OR Prunella OR Pyrrhula OR Rattus OR Regulus OR Rhinolophus OR Riparia OR Rupicapra OR Saxicola OR Sciurus OR Scolopax OR Sicista OR Sitta OR Sorex OR Spalax OR Spermophilus OR Streptopelia OR Strix OR Sturnus OR Sus OR Sylvia OR Talpa OR Tamias OR Testudo OR Tetrao OR Timon OR Troglodytes OR Turdus OR Tyto OR Upupa OR Ursus OR Vanellus OR Vipera OR Vulpes OR Zootoca)

Genus nomenclature has been stable in Europe since 1945 and for those genus names that did change, we used both the old and the new genus name. As restrictions in PubMed we looked only for the publications for which full texts were available, and that pertained to animal species. For the publications that seemed to contain relevant data judged on their title, keywords and abstract, the full text version was downloaded. Their bibliographies were screened for citations not identified in the initial step of the literature search to get as complete a database as possible. 
Chapter 2 


\section{Chapter 3}

\section{Geodemographic analysis}

\section{of Borrelia burgdorferi sensu lato}

\section{using the 5S-23S rDNA spacer region}

This chapter has been published as:

Coipan, E.C., Fonville, M., Tijsse-Klasen, E., van der Giessen, J.W., Takken, W., Sprong, H., Takumi, K., 2013. Geodemographic analysis of Borrelia burgdorferi sensu lato using the 5S-23S rDNA spacer region. Infection, genetics and evolution: journal of molecular epidemiology and evolutionary genetics in infectious diseases 17, 216-222. 


\section{Abstract}

Background: Lyme borreliosis is the predominant tick-borne disease in the Northern hemisphere, with considerable heterogeneity in clinical manifestations. Here, we evaluated one genetic marker for its use in population genetic based analysis. For that we collected molecular and epidemiological records of Borrelia burgdorferi sensu lato isolates from ticks, animals and humans at various sites in The Netherlands and worldwide.

Methods: The 5S-23S rDNA (rrfA-rrlB) intergenic spacer region (IGS) from 291 Dutch Borrelia positive ticks was sequenced and compared to Borrelia sequences from GenBank. We estimated several population genetic measures to test the neutrality of the marker. We also assessed the ability of this marker to discriminate between Eurasian Borrelieae at a finer geographical resolution, and to detect population expansion per genospecies.

Results: The most prevalent genospecies in The Netherlands was B. afzelii, whereas $B$. garinii, B. burgdorferi sensu stricto, B. spielmanii and B. valaisiana were found less frequently. The result of the Ewens-Watterson-Slatkin test was consistent with neutral selection of IGS region. Estimated pairwise Fixation indices (Fst) were significantly different from zero between The Netherlands, the rest of Europe, Russia and Asia for B. afzelii and B. garinii. Estimated Fu's Fs were significantly negative for $B$. afzelii and $B$. garinii.

Conclusions: At least seven B. burgdorferi s.l. genospecies circulate in the Ixodes ricinus population in The Netherlands. The population genetic analyses of the IGS region can resolve subpopulations within a genospecies and detect a large excess of rare genetic variants at the genospecies level. A genetic trace of population expansion for $B$. afzelii and $B$. garinii is consistent with the reported increase in Lyme borreliosis incidence in European countries. 


\section{Introduction}

The most prevalent and widespread vector-borne infection in the Northern hemisphere is Lyme borreliosis, an infectious disease caused by a group of spirochetes classified as Borrelia burgdorferi sensu lato. Molecular and phenotypic studies showed that the polymorphism of these bacteria far exceeds intra-species variability (Derdakova and Lencakova 2005). As a result, the $B$. burgdorferi sensu lato complex has been subdivided into at least 18 different taxonomic entities or genospecies (Margos et al. 2011). This high degree of genetic variation is one explanation for the variability in the clinical aspects of Lyme borreliosis. A possible correlation between genospecies and clinical symptoms has been proposed: arthritis and carditis are thought to be preferentially associated with $B$. burgdorferi sensu stricto, the degenerative skin disorder acrodermatitis chronica atrophicans is primarily associated with $B$. afzelii, while neuroborreliosis is primarily associated with $B$. garinii (Balmelli and Piffaretti 1995,Lesnik et al. 1997,Lunemann et al. 2001,van Dam et al. 1993). However, exceptions have often been observed, indicating that (sub)species belonging to a single genospecies may be more heterogeneous than expected (Eiffert et al. 1995,Ryffel et al. 1999,Schaarschmidt et al. 2001,Wang et al. 1999b).

Absence of a strict association is not limited to the clinical manifestations only. B. burgdorferi s.l. is maintained in nature through enzootic cycles, which involve several ixodid tick species as vectors and a variety of warm and coldblooded vertebrate hosts. In Europe, the predominant vector of Lyme borreliosis is the tick Ixodes ricinus (Burgdorfer et al. 1983). Specific associations were observed between vertebrates and $B$. burgdorferi s.l. genospecies, for example between rodents, $B$. afzelii and $B$. burgdorferi sensu stricto, and between birds, $B$. garinii and $B$. valaisiana. The complement system present in the blood of the hosts is thought to be one of the active components in the Borrelia host specificity (Bhide et al. 2005,Kurtenbach et al. 2006a,Pausa et al. 2003). However, some studies showed that nonspecific enzootic cycles of Borrelia genospecies involving a group of atypical host species may also occur (Humair and Gern 2000, Humair et al. 2007,Moran Cadenas et al. 2007).

Apparent lack of a strict association between Borrelia genospecies, clinical aspects of Lyme borreliosis and host-specificities lingers for a fitting explanation. One tool to probe the association is a molecular marker that can distinguish Borrelia burgdorferi s.l. not only at the genospecies level but also possesses a high resolution to differentiate subpopulations within a single genospecies, defined according to sampling time, geographical location, or mammalian hosts. Our PCR-based detection and typing method utilizes the 5S-23S rDNA (rrfA-rrlB) 
intergenic spacer region (IGS), a non-coding segment located on the chromosome (Gazumyan et al. 1994). This spacer region is a frequently used molecular marker for classifying B. burgdorferi s.l. genospecies and identifying haplotypes at small geographic scale (Derdakova et al. 2003, Fingerle et al. 2008,Pecchioli et al. 2007). For example, based on this marker a B. afzelii-like genotype was found in The Netherlands and the Baltic regions of Russia and it was designated as B. ruski based on its unique IGS sequence (Alekseev et al. 2001,van Overbeek et al. 2008, Wielinga et al. 2006).

We evaluated this molecular marker's suitability for the study of the population dynamics of the spirochete. For this purpose, we made a database comprising molecular and geographical data of more than 2000 B. burgdorferi s.l. positive samples, mostly ticks (1719), but also from wildlife (213) and humans (149). More than half of the data ( 1250 samples) have been collected from literature and GenBank. As a minimal requirement, the marker should identify Borrelia genospecies circulating in The Netherlands, in a manner comparable to the existing multi-locus sequence typing scheme. The genetic marker should also be able to detect the existence of subpopulations within a Borrelia genospecies. Thus, considering Eurasia not as a single continuous population of a Borrelia genospecies, but rather as a set of subpopulations, each evolving in distinct smaller scale geographical regions, we test whether the IGS can detect "isolation by distance" in an area that is otherwise considered geographically continuous. Namely, we tested the ability of this molecular marker for discriminating between Borrelieae at a finer geographical resolution. Another important feature of a genetic marker is that it should not be biased by its involvement in a specific clinical manifestation in Lyme borreliosis patients. In other words, it should be under neutral selection. Lastly, the marker should reflect a detectable change in the population dynamics of Borrelia genospecies in the wild. We assessed the latter three criteria by calculating some population genetic measures.

\section{Materials and Methods}

\subsection{Sample collection and PCR based sequence analysis}

Over 6000 Ixodes ricinus nymphs and adults were collected between the years 2000 and 2011 by flagging the vegetation at 15 different forested locations spread throughout The Netherlands (Gassner et al. 2011,Sprong et al. 2012b,Sprong et al. 2009,Tijsse-Klasen et al. 2011a,Tijsse-Klasen et al. 2011b,Tijsse-Klasen et al. 2010). These locations were selected based on Lyme borreliosis incidence in humans as well as on the availability of ticks. Almost 300 ticks were derived from a retrospective tick-bite study in The Netherlands 
(Tijsse-Klasen et al. 2011c). The variable 5S-23S intergenic spacer region (IGS) was amplified by PCR with the HotStarTaq master mix (Qiagen, Venlo, The Netherlands) using 5'-TCAGGGTACTTAGATGGTTCACTTCC and 5'-BiotinGAGTTCGCGGGAGAGTAGGTTATT with the following conditions: 15 min $94^{\circ} \mathrm{C}$, then cycles of $20 \mathrm{~s} 94^{\circ} \mathrm{C}, 30 \mathrm{~s} 70^{\circ} \mathrm{C}, 30 \mathrm{~s} 72^{\circ} \mathrm{C}$ lowering the annealing temperature $1^{\circ} \mathrm{C}$ each cycle until reaching $60^{\circ} \mathrm{C}$, then 40 cycles at this annealing temperature and ending by $7 \mathrm{~min} 72^{\circ} \mathrm{C}$. For Reverse Line Blot (RLB) analysis of the PCR product the following amino labeled probes were used: $5^{\prime}$ CTTTGACCATATTTTTATCTTCCA (catch all), 5'-CTTCCATCTCTATTTAGCCAATTT (catch all), 5'-TATTTTTATCTTCCATCTCTATTTT (catch all), 5'AACACCAATATTTAAAAAACATAA (B. burgdorferi sensu stricto), 5'AACATGAACATCTAAAAACATAAA (B. garinii), 5'-AACATTTAAAAAATAAATTCAAGG (B. afzelii), 5'-GAATAAAACATTCAAATAATATAAAC (B. ruski), 5'CATTAAAAAAATATAAAAAATAAATTTAAGG (B. valaisiana), and 5'CAAAAAAATGAACATTTAAAAAAC ( $B$. lusitaniae). The recently identified $B$. bavariensis reacted consistently with our B. garinii probe (Margos et al. 2009). Thus, based on the RLB-analysis $B$. garinii and $B$. bavariensis positive samples were grouped. To minimize cross contamination and false-positive results, positive ( $p C R I I-T O P O$ vector with an IGS insert from each of the genospecies) and negative controls were included in each batch tested by PCR and RLB. In addition, DNA extraction, PCR mix preparation, sample addition, and PCR analysis were performed in separate, dedicated laboratories. A random selection of 291 positive PCR amplicons (Table 1) from all B. burgdorferi s.l. groups, including the ones that bound solely to the "catch-all" probes but excluding the ones which bound to more than one of the genus-specific probes (double infections), were purified with the Qiaquick gel extraction kit (Qiagen Inc.) and sequenced using an ABI PRISM BigDye Terminator Cycle sequencing Ready Reaction kit (Perkin Elmer, Applied Biosystems). Sequences were confirmed by sequencing both strands (Sanger et al. 1977). A further random selection of thirty-one samples out of the 291 PCR amplicons were sequenced and typed using the multilocus sequencing typing procedure (MLST) as previously described (Margos et al. 2008).

\subsection{Additional data collection}

In addition to the DNA sequences produced for this study, IGS sequences of Borrelia with minimal epidemiological data (geographic location, year, host species) were also retrieved from the GenBank database. Borrelia sequences that were too short or lacked relevant epidemiological data were omitted from further analyses. All molecular epidemiological data were stored in BioNumerics (Version 6.6; Applied Math, Belgium). 


\subsection{Genospecies identification}

For genospecies identification using the spacer region, a pairwise alignment was generated using Unweigthed Pair Group Method with Arithmetic mean (UPGMA) with a gap penalty (300\%) and gap-extension penalty (30\%) allowing no more than three insertions. The secondary criterion - "most identical matches" - was applied if two equivalent solutions had emerged from the first criterion (transitivity dilemmas). Thirty-one samples from the terminal branches of the resulting IGS dendrogram (Figure 1) were subjected to MLSTanalyses (Margos et al. 2008). The genospecies of these samples were identified by comparison of their MLST sequence to reference strains, which were retrieved from http://borrelia.mlst.net/ (Margos et al. 2008). A distance-based analysis was conducted for the concatenated housekeeping genes of the MLST sequences using Kimura 2-parameters distance estimates and trees were constructed using the Neighbour-Joining algorithm, implemented in the BioNumerics software version 6.6. Bootstrap proportions were calculated by the analysis of 1000 replicates for $\mathrm{NJ}$ trees.

Table 1. Summary of the Borrelia positive samples used for genetic population analyses.

\begin{tabular}{|c|c|c|c|c|c|c|}
\hline Geographical & & & Samples & Japlotype & & \\
\hline Genospecies & NL & EU & RU & AS & Others & Total \\
\hline B. afzelii & $146(32)$ & $32(17)$ & $123(40)$ & $40(22)$ & & $341(87)$ \\
\hline B. burgdorferi s.s. & $37(10)$ & $62(16)$ & $5(4)$ & $7(3)$ & $162(26)^{\mathrm{NA}}$ & $273(48)$ \\
\hline B. garinii & $53(18)$ & $69(21)$ & $101(64)$ & $70(43)$ & & $293(118)$ \\
\hline B. Iusitaniae & $3(2)$ & $42(24)$ & $2(2)$ & & $52(9)^{A F}$ & $99(31)$ \\
\hline B. valaisiana & $39(11)$ & $43(12)$ & $2(2)$ & $61(21)$ & & $145(41)$ \\
\hline B. spielmanii & $13(3)$ & $16(4)$ & $2(2)$ & & & $31(6)$ \\
\hline All & $291(76)$ & $264(94)$ & $235(114)$ & $178(89)$ & & $1182(325)$ \\
\hline
\end{tabular}

$\mathrm{NL}=$ The Netherlands; $\mathrm{EU}=$ Europe; $\mathrm{RU}$ = Russia; $\mathrm{AS}=$ Asia; NA = North America; $\mathrm{AF}=$ Africa 


\subsection{Haplotype analysis}

A haplotype was defined here as a group of Borrelieae within a genospecies in which all members share an identical sequence. For this, multiple DNA sequences from each genospecies were aligned using MAFFT (Katoh and Toh 2010) and trimmed to nucleotides between position 438843 and 439146 of whole genome sequence of $B$. afzelii strain PKo (GenBank entry CP002933). A multiple sequence-based cluster analysis was performed using UPGMA with a gap penalty of $300 \%$ and a gap-extension penalty of $30 \%$. Population genetic measures (Fst, Ewens-Watterson test, Tajima's D, Fu's Fs) were calculated using Arlequin (Excoffier and Lischer 2010). We calculated folded site frequency spectrum by analysing the sites where two different nucleotides appear in the alignment of the sample sequences. At each of these sites, we counted the number of times less frequent nucleotide (derived mutation) appears in the sample. Fay and $\mathrm{Wu}^{\prime} \mathrm{s}$ test was run from http://www.genetics.wustl.edu/jflab/htest.html (Fay and Wu 2000). To support the results obtained by IGS analysis we performed a similar analysis on 153 flagellin $B$ gene ( $f l a B$ ) sequences retrieved from GenBank and trimmed to nucleotides between positions 147722 and 147975 of the same B. afzelii PKo strain (GenBank entry CP002933).

\section{Results}

\subsection{Identification of Borrelia genospecies circulating in The Netherlands}

The $B$. burgdorferi s.l. infection of 6025 questing Ixodes ricinus nymphs and adults was monitored between the year 2000 and 2011 in several different studies conducted in The Netherlands. All ticks were tested by PCR followed by RLB with genospecies-specific probes. The overall Borrelia infection rate was $13 \%$ $(n=798)$. Based on the RLB analyses, the Borrelia-positive ticks comprised of $B$. afzelii and B. ruski $61 \%(n=482), B$. garinii and B. bavariensis $11 \%(n=90), B$. valaisiana $8.4 \%(n=67), B$. burgdorferi s.s. $2 \%(n=16), B$. lusitaniae $0.4 \%(n=3)$ and Borrelia positive samples only reacting with the "catch all" probes $18 \%(n=140)$. Approximately half of the Borrelia-positive tick lysates of each group were subjected to DNA sequence analyses of the IGS-fragment. Pairwise distancebased cluster analysis of the 291 successfully generated IGS sequences revealed thirteen different clusters (Figure 1). Sixty of these sequences were identical or highly similar to the previously identified B. ruski (Alekseev et al. 2001,van Overbeek et al. 2008, Wielinga et al. 2006). In order to genetically identify the genospecies of each of these clusters, several members of each cluster were subjected to MLST-analysis with comparison to reference strains. The phylogenetic analysis of the IGS was largely consistent with the MLST based 
analysis except for the following aspects: MLST-analysis revealed that $B$. ruski was indistinguishable from $B$. afzelii, whereas it generates two genetically different groups in the IGS analysis (Figure 1). Similar but less pronounced situations were found for the IGS clusters within B. spielmanii and B. burgdorferi s.s. Conversely, the MLST analysis clearly distinguished $B$. bavariensis from $B$. garinii (Margos et al. 2009), whereas in the IGS analysis the B. bavariensis cluster intercalated between the $B$. garinii clusters (Figure 1 ). The $B$. garinii and $B$. bavariensis IGS sequences differed by two nucleotide positions in the multiple alignment.
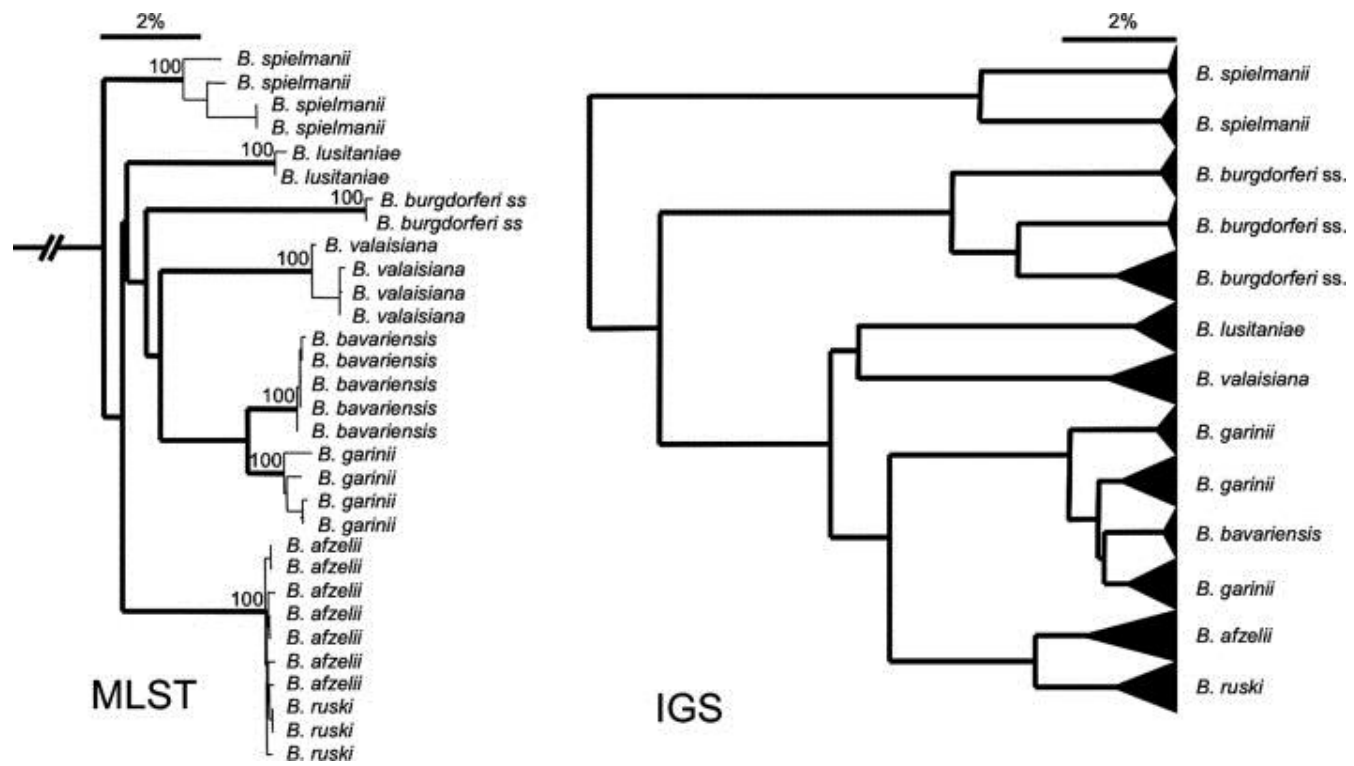

Figure 1. Cluster analysis of Borrelia isolates using MLST and IGS sequences. A neighbour-joining tree was based on the concatenated sequences of the eight fragments of housekeeping genes of 31 B. burgdorferi s.l. isolates from Dutch ticks, representing each genospecies. Only bootstrap values of $100 \%$ are indicated (MLST). A dendrogram showing the pairwise cluster analysis of the 291 IGS sequences of Borrelia isolates used in study. Terminal branches were collapsed for clarity (IGS).

\subsection{Genetic differentiation at subpopulation level}

The ability to differentiate subpopulations within a Borrelia genospecies is an epidemiologically relevant property of a molecular marker. As a test ground for IGS to differentiate subpopulations, we grouped the major Borrelia genospecies according to the six areas from where the data were collected (The Netherlands, the rest of Europe, Russia, Asia, North America, Africa) and 
assessed genetic heterogeneities within individual genospecies between these areas. Overall, in the sample of 1182 IGS sequences, 325 unique haplotypes were identified (Table 1).

\subsection{Genetic differentiation at subpopulation level}

The ability to differentiate subpopulations within a Borrelia genospecies is an epidemiologically relevant property of a molecular marker. As a test ground for IGS to differentiate subpopulations, we grouped the major Borrelia genospecies according to the six areas from where the data were collected (The Netherlands, the rest of Europe, Russia, Asia, North America, Africa) and assessed genetic heterogeneities within individual genospecies between these areas. Overall, in the sample of 1182 IGS sequences, 325 unique haplotypes were identified (Table 1).

When we visualized haplotype genealogies (Figure 2), we observed a clear molecular divergence of $B$. valaisiana between Asia and the remaining regions. That was likely due to isolation by distance of the common ancestor of the $B$. valaisiana samples, an event that occurred at some point of time in the past. Phylogenetic analysis of the IGS region alone and multilocus sequence typing (Chu et al. 2008, Hiraoka et al. 2007) indicate that the genetic differences between these two $B$. valaisiana groups are high. Recently, B. valaisiana from Asia has been proposed as a different genospecies, namely $B$. yangtze (Chu et al. 2008). Within B. garinii, the haplotype that prevails most evenly among the four sampling areas appeared to have diverged into at least two directions in the past: to Asia and Russia and to Europe including The Netherlands (Figure 2). To confirm these visual inspections and to detect other imperceptible molecular divergence, we used the Fst statistics to test the probable 'isolation by distance' evolutionary scenario (Wright 1943) within individual genospecies.

Estimated pairwise Fst values were significantly greater than zero between any pair of sampling areas for $B$. afzelii (Table 2 ). This demonstrates the ability of IGS to differentiate subpopulations within $B$. afzelii genospecies. The same test was applied to three additional Borrelia genospecies (B. garinii, B. valaisiana and B. burgdorferi s.s.); estimated pairwise Fst values were significantly greater than zero between a majority of sampling areas (Table 2). Exceptions were observed for some B. garinii, B. valaisiana and B. burgdorferi s.s. positive samples (Table 2). All Fst values were indistinguishable from zero for $B$. spielmanii and $B$. lusitaniae (Table 2), showing that IGS in our database failed to differentiate subpopulations within these two Borrelia genospecies. 

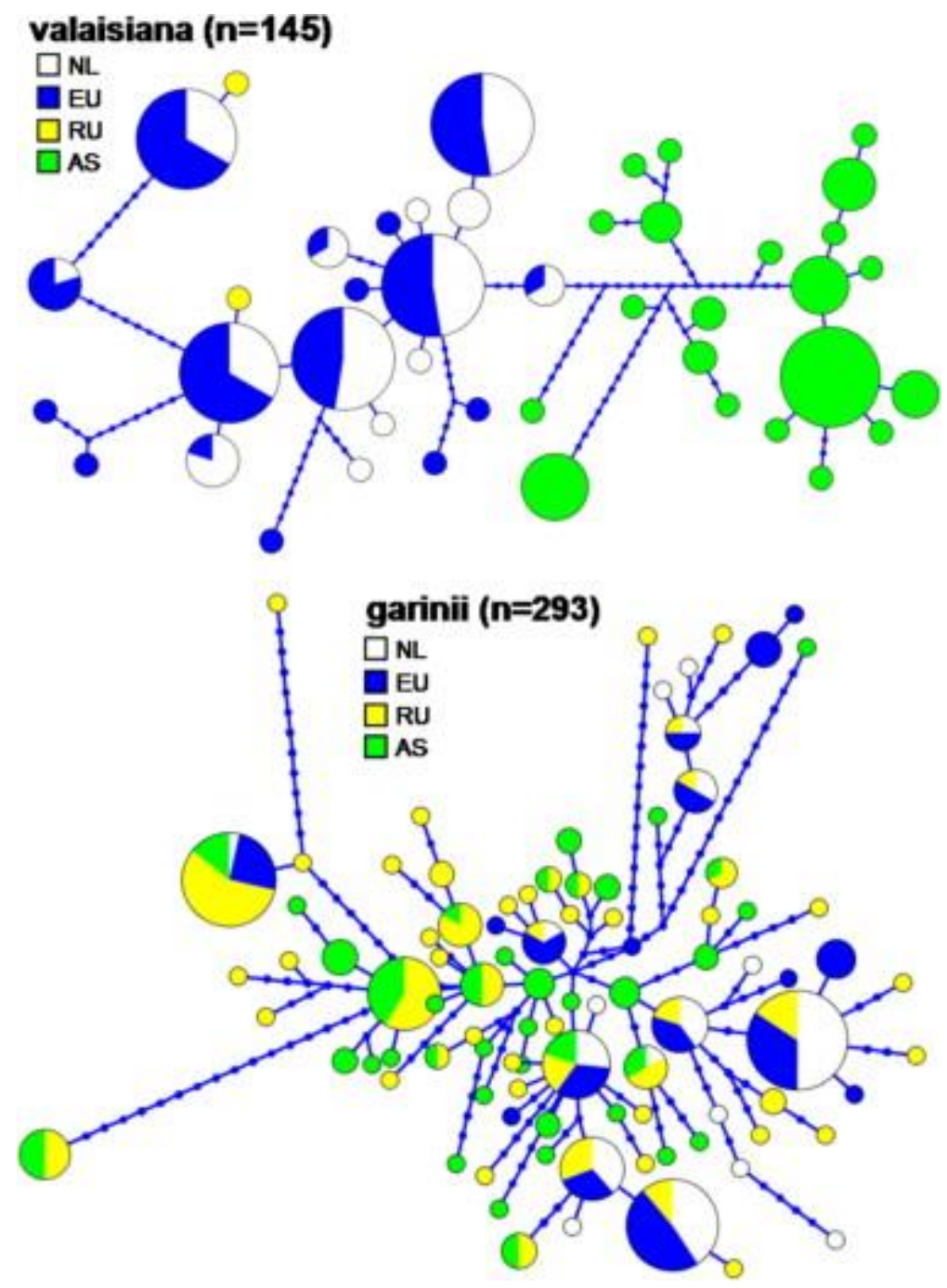

Figure 2. Haplotype genealogies of B. valaisiana and B. garinii. An open circle refers to a haplotype; its occurrence in the sample is proportional to the diameter. A colour refers to a sampling area. Sampling areas are abbreviated as in Table 1. A series of small blue dots connecting two haplotypes refer to unsampled haplotypes. The haplotype genealogies were made using Haploviewer software (Salzburger et al. 2011). 
Table 2. Differentiation indices in B. burgdorferi s.I. populations.

Fst is a measure of molecular divergence between subpopulations. It takes into consideration the following two events: (1) a pair of two sequences from a subpopulation are identical, and (2) a pair of two sequences from the collection of two subpopulations are identical (Slatkin 1991). Values with $\mathrm{p}<0.05$ are marked in bold and with an asterisk, and indicate a significant divergence.

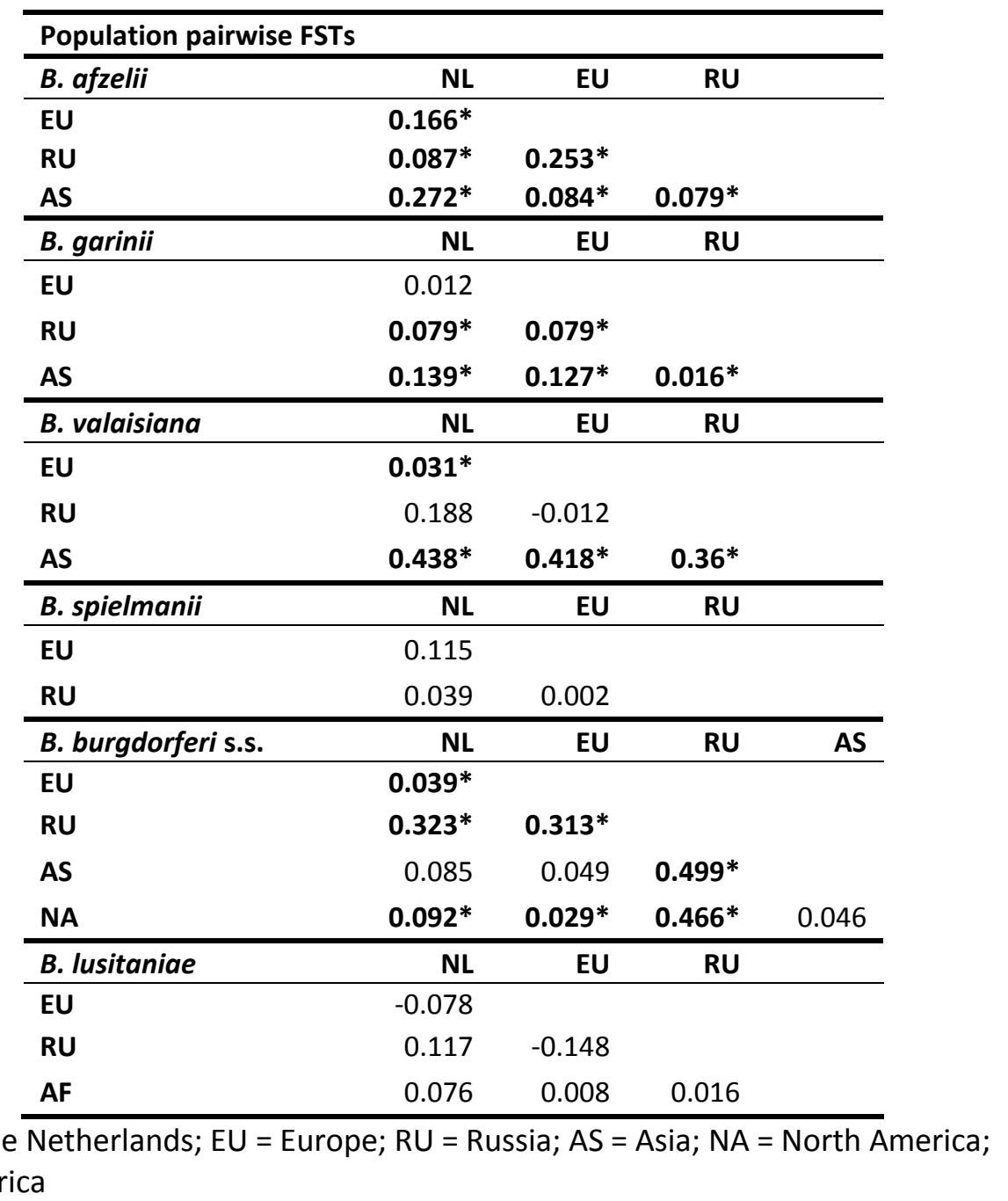


Table 3. Ewens-Watterson test of neutrality
B. afzelii
B. garinii
B. burgdorferi s.s.

\begin{tabular}{lcccccr}
\hline Statistics & Mean & s.d. & Mean & s.d. & Mean & s.d. \\
\hline Sample size & 85.25 & 57.73 & 73.25 & 20.07 & 54.6 & 64.45 \\
No. of alleles & 27.75 & 10.28 & 36.5 & 21.46 & 11.8 & 9.5 \\
Observed F value & 0.09 & 0.04 & 0.08 & 0.06 & 0.28 & 0.16 \\
Expected F value & 0.07 & 0.02 & 0.07 & 0.04 & 0.23 & 0.14 \\
Watterson test & 0.83 & 0.12 & 0.77 & 0.25 & 0.88 & 0.16 \\
Slatkin's exact test P-value & 0.92 & 0.11 & 0.78 & 0.24 & 0.91 & 0.13 \\
\hline
\end{tabular}

\subsection{Population expansion per genospecies}

A large excess in rare genetic variants is consistent with an expanding population. Hence, it is a relevant measure for a change in the transmission dynamics of Borrelia in the wild. To test whether IGS sequences contain any information regarding the population dynamics in the past, we estimated several population genetic measures, first of which was Ewens-Watterson-Slatkin test of neutrality (Ewens 1972,Slatkin 1994,Watterson 1977). The F-value (the probability that two IGS sequences randomly chosen from the samples share identical haplotype) agreed to the expectation (Table 3), supporting that this genetic marker is under neutral selection. Due to the limited number of samples, it was not possible to apply the neutrality test for B. lusitaniae, B. valaisiana and B. spielmanii.

Two other population genetic measures were Tajima's $D$ and Fu's Fs. Estimates of Fu's Fs were significantly negative for $B$. afzelii $(p=0.02)$ and $B$. garinii $(p=0.03)$ from The Netherlands (Table $4 A)$. These results demonstrate a large excess of rare genetic variants in the Dutch samples, over the expected genetic variants under the hypothesis of neutral selection and a constant population size. Analyses of four other genospecies (B. burgdorferi s.s., $B$. lusitaniae, B. valaisiana and B. spielmanii, Table 4A) did not support departure from the same hypothesis. Estimates of Tajima's $D$ were not significantly different from zero for any of the six genospecies in The Netherlands (Table 4A). When all positive samples from The Netherlands, the rest of Europe, Russia, and 
Asia were included in the same population genetic analyses, estimates for Fu's Fs remained significantly negative both for $B$. afzelii $(p=0.01)$ and $B$. garinii $(p=$ 0.05) (Table 4B). Estimates for Fu's Fs remained indistinguishable from zero for the other four genospecies (Table 4B).

Since population expansion is likely to leave its demographic trace also on other parts of the spirochete's genome, we used flagellin B gene from European $B$. afzelii and $B$. garinii to estimate the same population genetic measures. Estimates of Fu's Fs were significantly negative for both $B$. afzelii $(-7.4, p=0.007$, $\mathrm{n}=71)$ and $B$. garinii $(-25.8, \mathrm{p}<0.001, \mathrm{n}=82)$. Estimates of Tajima's $D$ were as well significantly negative for both $B$. afzelii $(-2.3, p=0.001)$ and $B$. garinii $(-2.0, p=$ 0.002 ). Hence, rare genetic variants are present in excess in the European samples at the independent locus flagellin B, confirming the results of the population genetic analyses based on IGS.

Table 4. Estimates of population genetic measures for six Borrelia genospecies based on IGS

\begin{tabular}{|c|c|c|c|c|}
\hline \multicolumn{5}{|l|}{ A: Dutch samples } \\
\hline Genospecies & Tajima's D & P-value & $\mathrm{Fu}^{\prime} \mathbf{s} \mathrm{F}_{\mathrm{s}}$ & P-value \\
\hline B. afzelii & 0.22 & 0.60 & -9.56 & 0.02 \\
\hline B. burgdorferi s.s. & -0.78 & 0.23 & 1.17 & 0.73 \\
\hline B. garinii & -0.73 & 0.24 & -5.55 & 0.03 \\
\hline B. Iusitaniae & 0.00 & 0.98 & 0.20 & 0.38 \\
\hline B. valaisiana & -1.06 & 0.13 & -2.54 & 0.11 \\
\hline B. spielmanii & 2.39 & 0.99 & 7.47 & 0.99 \\
\hline \multicolumn{5}{|l|}{ B: All samples } \\
\hline Genospecies & Tajima's D & P-value & Fu's $F_{s}$ & P-value \\
\hline B. afzelii & -0.67 & 0.30 & -10.11 & 0.01 \\
\hline B. burgdorferi s.s. & -0.84 & 0.25 & -0.30 & 0.48 \\
\hline B. garinii & -1.21 & 0.13 & -14.42 & 0.05 \\
\hline B. Iusitaniae & -0.46 & 0.59 & 0.16 & 0.43 \\
\hline B. valaisiana & -0.75 & 0.39 & -0.74 & 0.35 \\
\hline B. spielmanii & 1.10 & 0.95 & 5.90 & 0.75 \\
\hline
\end{tabular}

A: Estimates were made based on the 291 samples in Table 1

$\mathrm{B}$ : Estimates were made based on the 1182 samples in Table 1 
Table 5. Folded site frequency spectrum of $B$. afzelii (Netherlands)

\begin{tabular}{l|l|l|l|l|l|l|l|l|l|l}
\hline $\begin{array}{l}\text { Number of sequences } \\
\text { with the derived } \\
\text { mutation }\end{array}$ & 1 & 2 & 3 & 5 & 17 & 22 & 52 & 55 & 60 & 62 \\
\hline Number of sites & 9 & 2 & 2 & 1 & 1 & 1 & 1 & 1 & 5 & 1 \\
\hline
\end{tabular}

The number of IGS sequences was 146 . The number of nucleotides per sequence was 233.

Besides expanding populations, selective sweep, also known as genetic hitchhiking (Smith and Haigh 1974), could be responsible for large excesses of rare genetic variants in the samples of IGS sequences. We assessed whether the selective sweep is an alternative possibility. For this, we applied Fay and Wu's test (Fay and Wu 2000) to the folded site frequency spectrum (Durrett 2008) of the IGS sequences from the Dutch $B$. afzelii (Table 5) and $B$. garinii (Table 6). The test outcome was not significant for $B$. afzelii $(H$-statistic $=0.66, p$-value $=0.78)$, nor for $B$. garinii ( $H$-statistic $=0.57, p$-value $=0.67)$. Hence, selective sweep is incompatible with the genetic variability of Dutch B. afzelii and B. garinii.

\section{Discussion}

Analyzing the 5S-23S intergenic spacer region (IGS), we identified thirteen clusters of Borrelia genospecies. Samples from these clusters were re-analyzed by a MLST analysis. The resulting clusters corresponded largely to the IGS-based clusters. The previously designated $B$. ruski cluster turned out to be similar to the MLST of $B$. afzelii (Figure $1 \mathrm{MLST}$ ). Hence, we concluded that $B$. ruski is not a separate genospecies, but that its members are $B$. afzelii, with a deviant IGS sequence. Albeit marginally, $B$. bavariensis could be distinguished from $B$. garinii by means of the IGS sequences, as the sequences of the two genospecies differ constantly by two nucleotides. Despite the lack of agreement between IGS and MLST for B. ruski and B. bavariensis, we have shown in this study that the IGS sequence can be used as a high resolution marker within a genospecies, when utilized in conjunction with the internationally accepted MLST-scheme for definitive genospecies identification in certain situations.

Neutrality is a ubiquitous assumption in population genetic calculations, including the following calculations in our study: a measure of molecular divergence between subpopulations within a genospecies, a test for a population expansion and a test for selective sweeps. Neutrality is an acceptable assumption since: (1) IGS is a non-coding segment of chromosomal genome and (2) the Ewens-Watterson-Slatkin test did not detect significant departure from 
neutrality. Having this baseline result, we were in position to detect genospeciesspecific population subdivision and population expansion.

Estimates of Fst were significantly different from zero for $B$. afzelii, supporting a molecular divergence. That was likely due to isolation by distance of the common ancestor of the $B$. afzelii samples; an event that occurred at some point of time in the past. In addition to $B$. afzelii, a statistically significant trace of 'isolation by distance' was detected among B. garinii also. Especially for these two genospecies, the molecular marker IGS possesses a high resolution to differentiate subpopulations within a single genospecies, which are defined according to geographical location.

Fu's Fs statistic detected genetic traces of demographic changes for $B$. afzelii and $B$. garinii isolated from ixodid ticks in The Netherlands. Fu's Fs is more sensitive than Tajima's D to an excess of rare genetic variants in the samples (Fu 1997), and this has proved to be true for our IGS datasets (Table 4A). The alternative explanation for negative Fu's Fs estimates - selective sweep Negative estimates for Fu's Fs are congruent with population expansion (Fu 1997), but also with selective sweeps (Smith and Haigh 1974). The hallmark of selective sweeps - a large excess of highly frequent genetic variants in the sample - could not be detected by Fay and Wu's test (Fay and Wu 2000) in our IGS datasets. Hence, we opt for the hypothesis that B. afzelii and B. garinii increased in population sizes. This might have occurred through an increase in either the population of ixodid ticks, or in the mammalian host species, or in both.

Over the last decade, the incidence of Lyme borreliosis has increased in Europe (Hubalek 2009), including The Netherlands where a long-term retrospective study among general practitioners has shown a continuing increase in general practitioner consultations for erythema migrans, a common sign of early Lyme borreliosis (Hofhuis et al. 2006). Independently, in our study, population genetic based analysis of IGS supports the concept of population expansion for $B$. afzelii and $B$. garinii. Thus, the reported increase in Lyme borreliosis incidence is consistent with the genetic trace of the microbial demography. The consistency between the human-side and the pathogen-side of the story appears not to be limited by national borders and seems to extend at least to a (sub)-continental scale (e.g. Europe).

Our evaluation of the molecular marker IGS for use in future population genetics investigations has resulted in three validated properties. First, IGS is under neutral selection. Second, IGS possesses a high spatial resolution to differentiate subpopulations within individual genospecies. Third, IGS possesses 
a high temporal resolution to detect a genospecies-specific population expansion, either in the ixodid ticks, or in the mammalian hosts. By virtue of these three properties, we made a step forward to link transmission dynamics of Lyme borreliosis' causative agents in the wild to its incidence in humans.

\section{Acknowledgements}

This study was financially supported by the Dutch Food and Consumer Product Safety Authority (NVWA). We thank all the members of our department, especially Arnout de Bruin, for helpful discussions. Hein Sprong is grateful to Dr. Jill Shephard (Murdoch University, Australia) for initial and invaluable support in setting up population genetics in our laboratory. 


\section{Chapter 4}

\section{Contribution of rodent species to maintenance of tick-borne pathogens in enzootic cycles}

This chapter has been submitted as:

E.C. Coipan, G. van Duijvendijk, A.I. Krawczyk, T.R. Hofmeester, A.N. Swart, K. Takumi, H. Sprong. Contribution of rodent species to maintenance of tick-borne pathogens in enzootic cycles. 


\section{Abstract}

Small mammals are essential in the enzootic cycle of many tick-borne pathogens (TBP). To understand their contribution to the dynamics of TBP, we compared the infection prevalence with Borrelia afzelii, Borrelia miyamotoi, Candidatus Neoehrlichia mikurensis, and Anaplasma phagocytophilum in Ixodes ricinus larvae that feed on them. While the larvae that fed on shrews tested negative for most investigated bacteria, those fed on bank voles and wood mice (the two main rodent species in Northwestern Europe) showed high infection prevalence. Neoehrlichia mikurensis prevalence was positively associated with B. afzelii. Mathematical models including tick burden and infection prevalence indicated that bank voles are better amplifiers of these two bacteria than wood mice. To test whether the rodents have also differential roles in maintaining the genetic diversity of TBP, we used $B$. afzelii as model system. Despite the high genetic diversity within $B$. afzelii, there was no difference between wood mice and bank voles in the number and types of $B$. afzelii haplotypes they transmit. Our study suggests that wood mice and bank voles differ in their contribution to the dynamics of $B$. afzelii, and possibly other TBP, in questing ticks but not in their contribution to the genetic diversity of these microorganisms. 


\section{Introduction}

Tick-borne diseases are the most prevalent vector-borne diseases in Europe with an estimated incidence of more than 85000 human cases (2011). Maintenance of tick-borne pathogens in ticks occurs transstadially and/or transovarially. While the pathogens that have transovarial transmission could be maintained, theoretically, indefinitely in tick populations, the ones that have only transstadial transmission are dependent on various vertebrate hosts for amplification (Barbour 2004). In Europe, the most abundant tick species transmitting tick-borne pathogens is Ixodes ricinus (Hofmeester et al. 2016,Lindgren and Jaenson 2006). Some of the most abundant hosts for Ixodes ricinus ticks are rodents (Hofmeester et al. 2016), which are reservoirs for several tick-borne pathogens such as Borrelia burgdorferi sensu lato (s.I.) and Candidatus Neoehrlichia mikurensis.

Bacteria belonging to the complex of Borrelia burgdorferi s.l. are responsible for causing Lyme borreliosis - the most common vector-borne disease in Europe. Borrelia afzelii is one of the genospecies that accounts for most Lyme borreliosis cases in Europe (Stanek et al. 2012). The population of $B$. afzelii is maintained in a transmission cycle involving $I$. ricinus ticks - in which it is also the most prevalent Borrelia genospecies (Rauter and Hartung 2005) - and rodents. These bacteria have a high genetic diversity, not only at intergenospecies level, but also at intragenospecies level. It has been postulated that this genetic diversity is at the base of the multiple clinical manifestations that the infection with these bacteria can display (Dykhuizen et al. 2008,Strle et al. 2011, Wormser et al. 2008). Furthermore, it has been hypothesized that the genetic diversity, for at least some of the genomic loci, is maintained by a multiple-niche polymorphism balancing selection (Brisson and Dykhuizen 2004). The existence of such a balancing selection would have direct repercussions on the fitness of the bacteria in different vertebrate hosts and would provide new opportunities for control of Lyme borreliosis by means of the "dilution effect" (States et al. 2014).

Candidatus Neoehrlichia mikurensis (from here on referred to as $N$. mikurensis) was first reported as human pathogen in 2010 (Fehr et al. 2010,von Loewenich et al. 2010,Welinder-Olsson et al. 2010) and rodents appear to be its reservoir (Andersson and Raberg 2011,Silaghi et al. 2012b). Little is known about the genetic diversity of $N$. mikurensis and to what extent it displays host specific genetic variation (Grankvist et al. 2015).

Borrelia miyamotoi is found in I. ricinus in Europe since 2002 (Fonville et al. 2014,Fraenkel et al. 2002,Gern et al. 2010, Richter et al. 2003, Wilhelmsson et 


\section{Chapter 4}

al. 2010), but it has been recognized as a human pathogen only recently, with some of the clinical cases reported in The Netherlands (Hovius et al. 2013) and Russia (Karan et al. 2010,Platonov et al. 2011). Rodents have been shown to be able to transmit the bacteria to ticks (Burri et al. 2014) but other animals were also found infected (Scott et al. 2010,Wodecka et al. 2014).

Anaplasma phagocytophilum is another tick-borne bacterium that can cause disease in humans and animals (Ismail et al. 2010,Stuen et al. 2013). Although it has been detected in a wide range of wildlife species (Jahfari et al. 2014,Stuen et al. 2013), it is seldom found in rodents (Jahfari et al. 2014). In this study we used $A$. phagocytophilum as an antipode for the bacteria that are known to be mainly transmitted by rodents.

The role of vertebrate hosts in maintaining microorganisms in enzootic cycles has both a qualitative and a quantitative component. The qualitative component refers to the type of microorganism they can transmit, such as various genospecies or various genotypes of the same genospecies (Brisson and Dykhuizen 2004). The quantitative component refers to the reservoir capacity how many of the ticks feeding on an infected host get infected.

The host community has been hypothesized to generate the intraspecific genetic diversity of $B$. burgdorferi s.l. by various mechanisms. One of them is the multiple niche polymorphism balancing selection that implies that various hosts can act as ecological niches for a subset of the genotypes of a species (Gliddon and Strobeck 1975,Levene 1953). The second mechanism is the negative frequency dependent polymorphism. This postulates that no genotype has a maximum fit within a certain host species but that initial infection of a host triggers an immune response that will be protective against subsequent infections with genetically similar bacterial genotypes (Barthold 1999,Gromko 1977). Thus, the genotype that is most abundant at some point in time will be gradually decreased in frequency by negative selection from the host, favouring another one to become more frequent; a temporal shift in the frequency of the various genotypes occurs in this manner.

Several studies have addressed the qualitative component of transmission of B. afzelii (Durand et al. 2015,Hellgren et al. 2011,Jacquot et al. 2014) by investigating the polymorphism of the outer surface protein $\mathrm{C}$ gene (ospC) and ribosomal protein $\mathrm{L} 2$ gene (rplB) in either skin biopsies from rodents, or questing I. ricinus. There are, however, some disavantages of these approaches: a) rodents could be infected by a certain Borrelia genotype, but might transmit it with different efficiencies to the ticks feeding on them, b) the presence of certain genotypes in questing ticks is no evidence of the source. The two 
European studies that investigated the genetic polymorphism of $B$. afzelii in fed larvae (Perez et al. 2011,Tonetti et al. 2015) highlighted the existence of coinfection with multiple ospC haplotypes but did not address the question of differential roles of rodent species in maintenance of $B$. afzelii genetic polymorphism. The only comparative studies on fed larvae from various rodent species were performed in the U.S. on B. burgdorferi sensu stricto (s.s.) (Brisson and Dykhuizen 2004,Mechai et al. 2016).

Some of the most abundant rodent species throughout Europe are Apodemus sp. mice and Myodes sp. voles (De Boer et al. 1993,Gassner et al. 2013,Piesman and Gern 2004b). These have been shown to be important reservoirs for B. afzelii (Hanincova et al. 2003a), N. mikurensis, and B. miyamotoi, but their relative importance has not yet been quantified. Using xenodiagnoses, Humair et al. (Humair et al. 1999) have found that the infection rate of $B$. afzelii in ticks fed on Myodes glareolus (bank vole) was higher than in ticks fed on Apodemus sylvaticus (wood mouse). However, to our knowledge, there are no studies on the differential reservoir capacity of these species in natural settings.

In this study, we tested the relative importance of some of the most abundant small mammals for B. afzelii, B. miyamotoi, N. mikurensis and $A$. phagocytophilum by measuring the infection prevalence of these microorganisms in larvae fed on them, as a proxy for the realized reservoir capacity. We also test the hypothesis of host driven multiple nichepolymorphism of $B$. afzelii by testing fed larvae collected from Apodemus sylvaticus and Myodes glareolus - the two most abundant rodent species in Northwestern Europe. For this, we have chosen two genes that encode for bacterial proteins which seem to elicit the immune response of the rodent host (Cassatt et al. 1998,Wilske et al. 1986): ospC and decorin binding protein A $(\mathrm{dbpA})$. Should the niche-polymorphism model be plausible, we would expect to find distinct variants of the genes encoding for these two proteins in the larvae collected from the two rodent species.

\section{Results}

Apodemus sylvaticus (wood mice), Myodes glareolus (bank voles), Microtus agrestis (field voles), Sorex araneus (common shrews) and Sorex minutus (pygmy shrews) were caught during field sampling performed at 12 different sites in The Netherlands in 2014.

Full body inspection for ticks was performed on 844 small mammals. The small mammals carried a total of 13,356 larval and 229 nymphal Ixodes ricinus, with variable infestation levels between the various species (Supplementary Table 3). Apodemus sylvaticus had the highest mean larval burden - 20 (s.d. 


\section{Chapter 4}

38.1), significantly higher than that of M. glareolus - 15.7 (s.d. 29) (likelihood ratio test $p=0.04)$. For $M i$. agrestis, the average larval burden (14.5, s.d. 58.7) was almost equal to that of $M$. glareolus but the small sample size of the first resulted in a lack of significance of the likelihood ratio test.

We tested for the presence of the microorganisms in 1,241 fed larvae from 95 small mammals (49 A. sylvaticus, $18 \mathrm{M}$. glareolus, $21 \mathrm{~S}$. araneus, two S. minutus and five Mi. agrestis). Borrelia burgdorferi s.l. was present in $16.9 \%$ $(210 / 1241)$ of the larvae, coming from $27.4 \%(26 / 95)$ of the vertebrates, $B$. miyamotoi was present in $2.2 \%$ (27/1241) of the larvae from $18.9 \%(18 / 95)$ of the vertebrates, and $N$. mikurensis was present in $26.9 \%$ (334/1278) of the larvae from $27.4 \%(26 / 95)$ of the vertebrates. Anaplasma phagocytophilum was present in $0.2 \%(3 / 1241)$ of the larvae from $3.16 \%(3 / 95)$ of the small mammals. All the $B$. burgdorferi s.l. typed by sequencing of IGS were identified as $B$. afzelii.

Multiple infections were common in larvae. Infections with two of the four microorganisms were found in 113 larvae, with three microorganisms in six of them, and with all four in one larva (Supplementary Table 4). We found coinfection with $B$. afzelii and $N$. mikurensis to be more prevalent than expected under the assumption of independent prevalences of two bacteria (Fisher's exact test $p<2.2 \mathrm{e}-16$; Supplementary Table 4 ).

The prevalence of infection was significantly higher in larvae fed on $M$. glareolus than on $A$. sylvaticus with both $B$. afzelii s.l. and N. mikurensis (both microorganisms, Fisher's exact test $\mathrm{p}$-value $=<2.2 \mathrm{e}-16$ ). There were no differences in infection with both bacteria between the two rodent species, although in our sample there were more $M$. glareolus individuals infected compared to $A$. sylvaticus. There were also no significant differences between the two main rodent species regarding the infection prevalence with $B$. miyamotoi and $A$. phagocytophilum.

Infection with at least one microorganism was found in larvae from $28 \%$ of rodents. The highest prevalence of infection was found on larvae feeding on $M$. glareolus $-63 \%$, while for $A$. sylvaticus it reached $25 \%$ (Table 1 ).

Negative-binomial models indicated that the larval burden of rodents is positively correlated with the nymphal burden (Table 2), namely, the rodents that have more nymphs have also more larvae.

The model containing all the parameters and larvae as explanatory variables was the one that explained best the differences in prevalence of infection (Table 2) for B. afzelii, with the rodent species being most strongly correlated with the infection prevalence of the larvae, followed by the sex of the 
rodent and the larval burden. Thus, Myodes rodents, male rodents, and rodents with a higher larval burden displayed a higher prevalence of infection with $B$. afzelii in the attached larvae. Contrary to our expectations, the nymphal burden was not significantly correlated with the prevalence of infection of larvae with $B$. afzelii.

Table 1. Tick burden and prevalence of infection of the five small mammals species. $p=$ prevalence of infection with the selected bacterium

\begin{tabular}{|c|c|c|c|c|c|c|}
\hline & \multirow{2}{*}{$\begin{array}{l}\text { Unit of } \\
\text { count }\end{array}$} & \multirow{2}{*}{$\begin{array}{l}\text { Larval/nymphal } \\
\text { tick burden } \\
\text { Median (range) }\end{array}$} & \multicolumn{4}{|c|}{$\mathrm{p}$ (Positive/tested) } \\
\hline & & & $\begin{array}{r}B . \\
\text { burgdorferi } \\
\text { s.l. }\end{array}$ & $\begin{array}{r}B . \\
\text { miyamotoi }\end{array}$ & $\begin{array}{r}N . \\
\text { mikurensis }\end{array}$ & $\begin{array}{r}A . \\
\text { phagocytophilum }\end{array}$ \\
\hline $\begin{array}{l}\text { A. } \\
\text { sylvaticus }\end{array}$ & $\begin{array}{l}\text { rodent } \\
\text { larva }\end{array}$ & $\begin{array}{r}25 / 0 \\
(1-226 / 0-7)\end{array}$ & $\begin{array}{r}0.34(17 / 49) \\
0.13 \\
(98 / 733)\end{array}$ & $\begin{array}{r}0.24 \\
(12 / 49) \\
0.02 \\
(19 / 733)\end{array}$ & $\begin{array}{r}0.38 \\
(19 / 49) \\
0.22 \\
(160 / 733)\end{array}$ & $\begin{array}{r}0.04(2 / 49) \\
0.003(2 / 733)\end{array}$ \\
\hline $\begin{array}{l}\text { M. } \\
\text { glareolus }\end{array}$ & $\begin{array}{l}\text { rodent } \\
\text { larva }\end{array}$ & $\begin{array}{r}10 / 0 \\
(2-125 / 0-15)\end{array}$ & $\begin{array}{r}0.33(6 / 18) \\
0.28 \\
(107 / 380) \\
\end{array}$ & $\begin{array}{r}0.16(4 / 18) \\
0.01 \\
(5 / 380) \\
\end{array}$ & $\begin{array}{r}0.38(7 / 18) \\
0.46 \\
(174 / 380) \\
\end{array}$ & $\begin{array}{r}0.05(1 / 18) \\
0.003(1 / 380) \\
\end{array}$ \\
\hline $\begin{array}{l}\text { Mi. } \\
\text { agrestis }\end{array}$ & $\begin{array}{l}\text { rodent } \\
\text { larva }\end{array}$ & $\begin{array}{r}30 / 2 \\
(2-386 / 1-9)\end{array}$ & $\begin{array}{r}0.6(3 / 5) \\
0.42(5 / 12) \\
\end{array}$ & $\begin{array}{r}0(0 / 5) \\
0(0 / 12) \\
\end{array}$ & $\begin{array}{r}0(0 / 5) \\
0(0 / 12) \\
\end{array}$ & $\begin{array}{r}0(0 / 5) \\
0(0 / 12) \\
\end{array}$ \\
\hline S. araneus & $\begin{array}{l}\text { rodent } \\
\text { larva }\end{array}$ & $2 / 0(0-28 / 0-1)$ & $\begin{array}{l}0(0 / 15) \\
0(0 / 113)\end{array}$ & $\begin{array}{r}0.14(1 / 15) \\
0.01 \\
(1 / 113)\end{array}$ & $0(0 / 15)$ & $0(0 / 15)$ \\
\hline S. minutus & $\begin{array}{l}\text { rodent } \\
\text { larva }\end{array}$ & $2.5 / 0(2-3 / 0-0)$ & $\begin{array}{l}0(0 / 2) \\
0(0 / 3)\end{array}$ & $\begin{array}{l}0(0 / 2) \\
0(0 / 3)\end{array}$ & $\begin{array}{l}0(0 / 2) \\
0(0 / 3)\end{array}$ & $\begin{array}{l}0(0 / 2) \\
0(0 / 3)\end{array}$ \\
\hline
\end{tabular}

For $N$. mikurensis the models with all three parameters explained best the differences in prevalence of infection in larvae (Table 2). In this case, the model that used nymphal burden as explanatory variable had a better fit (lower AIC) than that using the larval burden, although, in both models, the tick burdens were correlated with the prevalence of infection (Table 2). Myodes rodents, male rodents, and rodents with larger larval/nymphal burden were positively correlated with the infection prevalence. Neither the species, sex or weight of the rodents, nor their nymphal burden correlated with the infection prevalence for B. miyamotoi. There was a significant slightly negative correlation of the larval burden with the infection prevalence $(p$-value $=0.036)$.

Of the 217 qPCR positive larvae, 131 yielded a sequence for IGS, 144 for $\mathrm{dbpA}$ and 134 for ospC (sequences given in the associated dataset).

We identified 7 types of IGS, 9 types of $\mathrm{dbpA}$ and 7 types of ospC (Figure 1 ). Both rodent species comprised the majority of the sequence types (Figure 1 ). 


\section{Chapter 4}

For dbpA the exceptions were types 1 and 8 , which were absent in A. sylvaticus, and type 3, which was absent in M. glareolus, ospC type 7, which was absent in A. sylvaticus, and IGS type 6 which was absent in M. glareolus (Supplementary Table 5). For ospC six of our seven haplotypes were described in a recent article by Durand et al. (Durand et al. 2015). The seventh haplotype was also identified as identical with other Central European ospC sequences by using NCBI Blast. Similarly, IGS and dbpA have correspondents in other European countries (results not shown).

Table 2. Predictive parameters in the best performing binomial models for infection prevalence of larvae and negative-binomial models for larval and tick burdens of rodents. $\mathrm{p}=$ prevalence of larval infection with the selected bacterium ( $\mathrm{Bb}=$ Borrelia burgdorferi s.I., $\mathrm{Nm}=$ Neoehrlichia mikurensis)

\begin{tabular}{|c|c|c|c|c|c|}
\hline Model & term & estimate & std.error & statistic & p.value \\
\hline \multirow{4}{*}{$\begin{array}{l}\mathrm{p}(\mathrm{Bb}) \sim \text { Species + Sex + Larvae } \\
(\mathrm{df} . \text { resid = 45; AIC = 173.38) }\end{array}$} & (Intercept) & -3.90 & 0.38 & -10.37 & $3.57 \times 10^{-25}$ \\
\hline & Species $^{a}$ & 1.58 & 0.22 & 7.08 & $1.39 \times 10^{-12}$ \\
\hline & Sex ${ }^{b}$ & 1.00 & 0.40 & 2.48 & 0.01 \\
\hline & Larvae & 0.01 & 0.00 & 2.01 & 0.04 \\
\hline \multirow{4}{*}{$\begin{array}{l}\mathrm{p}(\mathrm{Nm}) \sim \text { Species + Sex + Larvae } \\
\text { (df.resid = 45; AIC = 411.28) }\end{array}$} & (Intercept) & -2.87 & 0.25 & -11.70 & $1.31 \times 10^{-31}$ \\
\hline & Species $^{a}$ & 1.39 & 0.17 & 8.26 & $1.50 \times 10^{-16}$ \\
\hline & Sex ${ }^{b}$ & 0.82 & 0.27 & 3.11 & $1.88 \times 10^{-3}$ \\
\hline & Larvae & 0.01 & 0.00 & 3.15 & $1.61 \times 10^{-3}$ \\
\hline \multirow{4}{*}{$\begin{array}{l}\mathrm{p}(\mathrm{Nm}) \sim \text { Species + Sex + Nymphs } \\
(\text { df.resid = 45; AIC = 351.30) }\end{array}$} & (Intercept) & -2.68 & 0.23 & -11.54 & $8.57 \times 10^{-31}$ \\
\hline & Species $^{a}$ & 1.18 & 0.17 & 6.80 & $1.06 \times 10^{-11}$ \\
\hline & Sex ${ }^{b}$ & 0.82 & 0.26 & 3.20 & $1.39 \times 10^{-3}$ \\
\hline & Nymphs & 0.15 & 0.02 & 7.59 & $3.16 \times 10^{-14}$ \\
\hline \multirow{2}{*}{$\begin{array}{l}\text { Nymphs } \sim \text { Larvae } \\
(\text { df.resid }=47 ; \text { AIC }=96.24)\end{array}$} & (Intercept) & -2.01 & 0.47 & -4.24 & $2.27 \times 10^{-5}$ \\
\hline & Larvae & 0.03 & 0.01 & 4.45 & $8.66 \times 10^{-6}$ \\
\hline
\end{tabular}

${ }^{a}$ The estimate gives the difference between Apodemus sylvaticus and Myodes glareolus, a positive estimate means that $M$. glareolus had a higher infection prevalence compared to $A$. sylvaticus.

${ }^{\mathrm{b}}$ The estimate gives the difference tween the sexes, a positive estimate means that males had a higher infection prevalence compared to females.

We noticed that the sequences containing ambiguous nucleotides had, in most of the cases, the double peaks at the same positions as the segregating sites in the unambiguous sequences. This implies that the ambiguous sequences were actually generated by the coexistence of the same haplotypes and are not new haplotypes.

Most of the rodents allowed transmission of multiple Borrelia haplotypes, with no difference between the two most common species in the mean number 
of haplotypes they can transmit ( $t$ test: IGS p-value $=0.07, \mathrm{dbpA} p$-value $=0.07$, ospC $p$-value $=0.13$ ). The AMOVA test indicated that there was no genetic differentiation between the $B$. afzelii genotypes carried by the two rodent species (Table 3) for any of the three investigated loci, the percentage of variation explained by the rodent species being less than $10 \%$. The largest percentage of the variation was found within the population of $B$. afzelii from one location only. There was also a relatively high variation between the various sampling locations.

Table 3. Results of AMOVA test for the three selected genes

\begin{tabular}{lccc}
\hline Gene & \multicolumn{3}{c}{ Percentage of variation } \\
\hline & Among species (df) & Among locations within species (df) & Within locations (df) \\
\cline { 2 - 4 } IGS & $-19.88(2)$ & $46.45(8)$ & $73.43(120)$ \\
dbpA & $-7.26(2)$ & $66.22(8)$ & $41.04(134)$ \\
ospC & $-21.74(2)$ & $54.43(8)$ & $67.31(123)$ \\
\hline
\end{tabular}

\section{Discussion}

In this study we compared the prevalence of infection of larvae feeding on the two most common rodent species in the Netherlands with $B$. afzelii, $B$. miyamotoi, and $N$. mikurensis, as a proxy for the realised reservoir capacity of these small mammals for the respective bacteria. This study adds to the current knowledge on the transmission capacity of the three pathogens using fieldcollected rodents and the larvae that are naturally attached to them. Previous studies on reservoir capacity of small mammals for these microorganisms were based either on xenodiagnosis (Humair et al. 1999), or on a very small number of rodents $(<5)$ (Burri et al. 2014).

One of the main assumptions of our study was that the larvae are free of the microorganisms we tested for, as the transovarial transmission is considered to be negligible (Richter et al. 2012, Rollend et al. 2013). A second assumption was that all larvae we recorded as fed had enough time to get infected with the tested microorganisms, thus not taking into account the effects of feeding duration on the transmission (Richter et al. 2012).

Borrelia afzelii and $N$. mikurensis occur together in larvae feeding on rodents more often than expected should they be independent of each other. Similar results were also found in questing ticks (Coipan et al. 2013c) as well as in voles (Andersson et al. 2014). This implies similar maintenance patterns in enzootic cycles. Additionally, co-infections can facilitate or hinder subsequent transmission of other pathogens, in vertebrate hosts as well as in humans (Belongia 2002,Ginsberg 2008, Hovius et al. 2008,Thomas et al. 2001). 


\section{Chapter 4}

We show that larvae feeding on $M$. glareolus rodents have a higher prevalence of infection with $B$. afzelii than those feeding on $A$. sylvaticus. Thus, our results confirm, with field derived data, what previous studies (Humair et al. 1999, Talleklint and Jaenson 1994) indicated based on xenodiagnoses, namely that bank voles are possibly better transmitters of $B$. afzelii than wood mice.

Furthermore, we show that the larvae that feed on $M$. glareolus have a higher infection prevalence also for $N$. mikurensis. Our models indicated that the prevalence of infection in the attached larvae is correlated not only with the rodent species but also with the sex of the rodents and the number of larvae or nymphs they carry. Additionally, we found the larval and nymphal burdens of the rodents to be correlated. Several studies have shown that the distribution of tick developmental stages on hosts is not independent but coincident (Ferreri et al. 2014,Perkins et al. 2003), meaning that the hosts that are infested by larvae would also host the greatest number of nymphs. For pathogens that have no transovarial transmission - such as $B$. afzelii, the nymphs feeding on the same rodents as the larvae are of foremost importance in transmission, since they are the ones that infect the rodent. Our models indicate that, indeed, the rodents hosting the highest number of larvae are the ones that also have more nymphs. On the other hand, the observed highly aggregated distribution of the ticks is the result of, among other factors, host exposure and host behaviour (Brunner and Ostfeld 2008) and sexually active male rodents, which also have the highest body mass, have been previously identified as highly parasitized (Perkins et al. 2003). This is in agreement with our findings that species, and sex are positively correlated with either the tick burden or the infection rate.

It seems that the relative importance of the two rodent species in maintaining these two pathogens is determined by the behaviour of the host. Thus, the prevalence of infection of the larvae feeding on $M$. glareolus could be explained by the higher weight of this rodent species, which could imply also higher metabolic rates, therefore the need for more food and an increased activity to search for it, which in turn leads to encountering more (infected) ticks.

Figure 1. Rooted phylogenetic tree of IGS with the sample names coloured according to the rodent genus from which the ticks were collected. The associated heatmap depicts the allele types of the three genes in the study: IGS, $\mathrm{dbpA}$, and ospC; each colour is a distinct allele type; white = lack of data; black = B. garinii used for rooting the tree $\rightarrow$ 


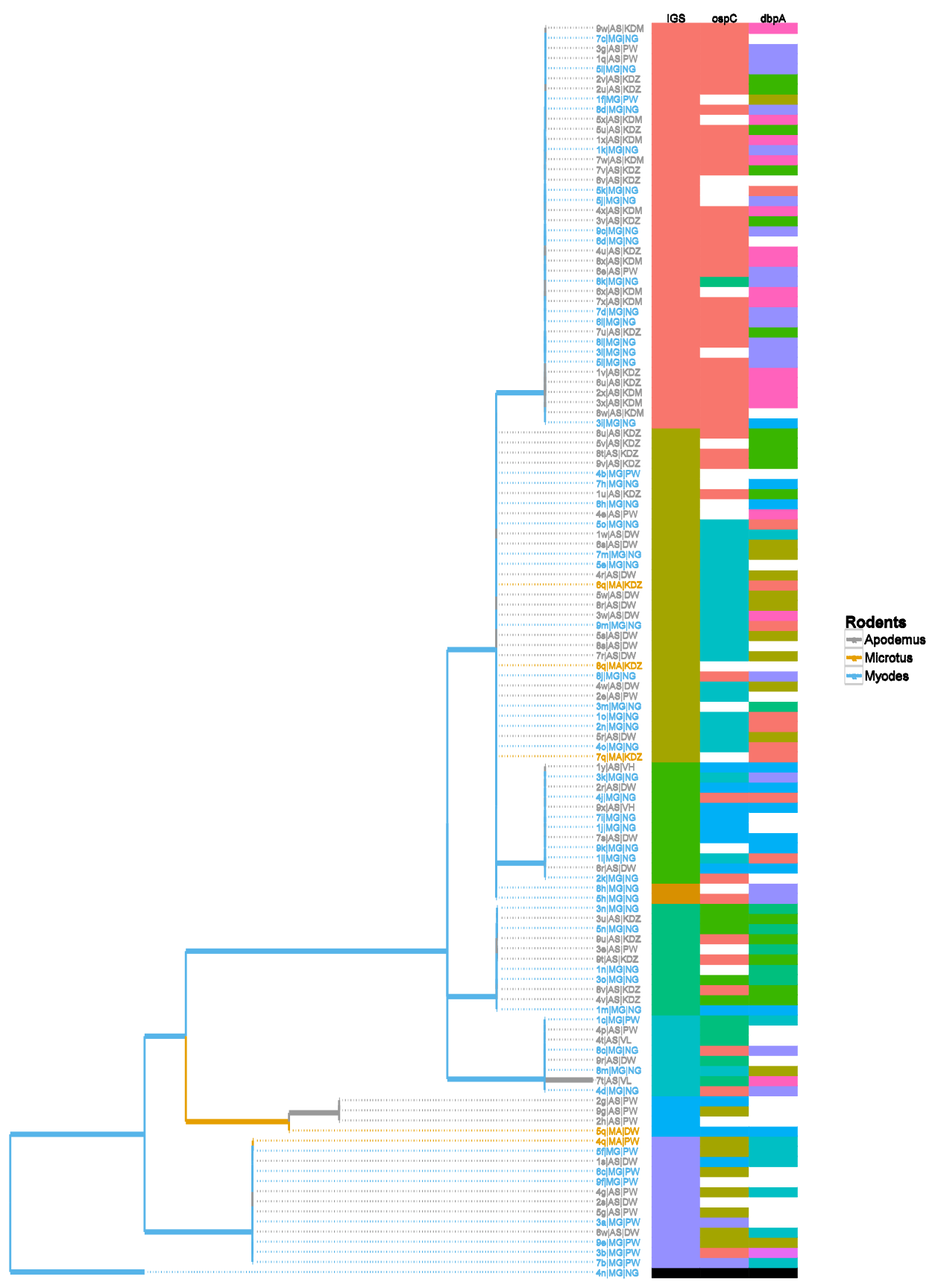


In view of these findings, we argue that $M$. glareolus might be a more important host in maintenance of $B$. afzelii in enzootic cycles than $A$. sylvaticus. Previous studies indicated that, although $M$. glareolus is a better transmitter, the difference between the two rodent species in producing infected nymphs could be attenuated by the higher tick burdens of $A$. sylvaticus (Humair et al. 1993a,Kurtenbach et al. 1995, Raberg 2012,Talleklint and Jaenson 1997). Although, in our study, just as in others, A. sylvaticus harboured, on average, more ticks than $M$. glareolus, when taking into account the prevalence of infection in the larvae feeding on the two, $M$. glareolus would still generate more infected engorged larvae than A. sylvaticus. Another factor that might compensate for the difference in infectivity between the two rodent species is the moulting success (Humair et al. 1999), which has been shown to be higher for larvae that feed on A. sylvaticus. However, recent studies (Burri et al. 2014) indicate that that holds true only for xenodiagnosis larvae and not for ticks caught from the wild. All these corroborated aspects suggest that $M$. glareolus could be the principal vertebrate host in maintaining $B$. afzelii and $N$. mikurensis.

There was no significant difference in the infectivity of the two rodent species with B. miyamotoi. One of the explanations could be the low numbers of positive larvae found on these rodents that would account for lack of statistical power. On the other hand, the relatively low acquisition of $B$. miyamotoi by ticks, which is consistent with the results of previous studies (Taylor et al. 2013), could be due to the fact that $B$. miyamotoi only causes a short-term systemic infection in rodents (Wagemakers et al. 2016). Furthermore, B. miyamotoi is known to have transovarial transmission in ticks (Rollend et al. 2013), which might introduce bias in the results of our analysis, as we cannot know whether the larvae were infected already before the blood meal on the rodent.

Another important finding of our study is that the two rodent species do not differ in the Borrelia genotypes that they transmit. There are a couple of hypotheses for maintenance of polymorphism at several highly variable genetic loci of Borrelia. One of these is that of niche-polymorphism balancing selection and it implies that "the different hosts are different niches" (Brisson and Dykhuizen 2004). This hypothesis has been tested on B. burgdorferi s.s. in North America. Previous studies on B. afzelii in Europe (Hellgren et al. 2011,Jacquot et al. 2014) focused only on tissues from the rodents. While this is a good approach to detect Borrelia genotypes that cause systemic infection in the rodents, it could miss the genotypes that are transmitted directly between the ticks by cofeeding, which, according to recent studies (Tonetti et al. 2015), could account for up to $66 \%$ of the $B$. afzelii transmission. 
Our analysis indicates that both $A$. sylvaticus and $M$. glareolus contribute to maintenance of all IGS, dbpA and ospC haplotypes (Figure 1). However, our sample size could have been insufficient to capture the whole spectrum of haplotypes, so that we cannot exclude the possibility that the absence or scarcity of a haplotype in a rodent species is not caused by the fact that the sampled rodents were infected with some haplotypes only, due to chance.

Unlike Hellgren et al. (2011) we did not detect geographic differentiation either at the three genetic loci (Table 3). Furthermore, most of the alleles that we found were also found in other parts of Europe. Thus, our results indicate that the geographic differentiation between $B$. afzelii strains at these three loci is an unfunded postulate. There are indeed differences in the frequencies with which the various haplotypes occur. Thus, while in Switzerland the most abundant ospC type was $\mathrm{A} 10$, followed at more than $30 \%$ difference by $A 9, A 1$, and A14 (Durand et al. 2015), in our study A3 and one as yet unnamed haplotype in the used nomenclature were the first two most abundant ospC types, while A9 and $\mathrm{A} 10$ came in the $3 \mathrm{rd}$ and 4 th positions. One of the explanations for this could be that, in the aforementioned study the samples represented the population of B. afzelii in questing ticks, to which other vertebrate hosts also contribute; it is, therefore, not excluded that, types $A 9$ and $A 10$ are maintained primarily by other vertebrates that would be more abundant in Central Europe. However, since in our study both rodent species showed almost equal infectivity for these haplotypes, we find this possibility a remote one. The second explanation, which we give more credit to, is that the polymorphism of ospC is maintained by a negative frequency-dependent mechanism (Gromko 1977), which allows for fluctuations in time of the alleles' frequencies and consequently for the shift in frequencies at different geographic locations.

We observed a high degree of linkage disequilibrium between the alleles at the three loci. That implies that, in spite of the fragmented genome of $B$. afzelii, and subsequent facility for gene exchange, the horizontal gene transfer is not a pervasive phenomenon in these bacteria. This could be another indication that these spirochetes have evolved to have equal fitness for both species of the main vertebrate hosts. Given the frequency of co-infection observed in the larvae feeding on rodents, there would be plenty of opportunities for lateral gene transfer, should one of the genotypes have an advantage in resisting the host's immune response. This implies that the innate immune response of the various small rodents does not exempt a strong selective pressure.

In this study we highlight the positive association of $B$. afzelii with $N$. mikurensis in larvae feeding on rodents and the similar mechanisms that are driving the prevalence of infection with these two pathogens in the larvae 
feeding on the two main rodent species. We consider this to be strong evidence of similar maintenance patterns of these pathogens in enzootic cycles. Our study emphasizes, thus, that in absence of detailed information on the ecology and genetics of emerging pathogens, comparative study with better known microorganisms can prove to be a very useful approach.

We show that small rodents differ only in their reservoir capacity for $B$. afzelii, but do not contribute differently to the genetic diversity of these bacteria at neither chromosomal nor plasmid loci. Thus, $M$. glareolus might be the best amplifier of $B$. afzelii and $N$. mikurensis in enzootic cycles. The results of the genetic analysis indicate that the existence of multiple haplotypes at plasmid loci that are responsible for the microorganism-host interaction is more likely to be the result of negative-frequency dependent selection rather than of nichepolymorphism balancing selection.

\section{Experimental procedure}

\section{Sample collection}

We trapped rodents and shrews with small mammal life-traps (Heslinga Traps, Groningen, The Netherlands) in July 2013-August 2014 in 12 different regions in The Netherlands. Each animal was thoroughly examined for ticks. All ticks were collected and placed in separate tubes. For each tick we registered the species, the development stage (larva, nymph, adult) and the feeding status (fed/unfed). The samples were stored at $-20^{\circ} \mathrm{C}$ until DNA extraction.

\section{DNA extraction and qPCR detection of microorganisms}

Total DNA was extracted from fed larvae only. This was done to ensure that the bacteria detected in the larvae came from the vertebrate hosts they were feeding on at that moment, considering that transovarial transmission of Neoehrlichia mikurensis and Borrelia burgdorferi s.l. is absent or very low (Silaghi et al. 2016,van Duijvendijk et al. 2016). The extraction was performed using the QIAGEN DNeasy 96 Blood \& Tissue Kit (QIAGEN, Venlo, The Netherlands).

For the detection of $B$. burgdorferi s.l. and B. miyamotoi, a triplex qPCR, targeting ospA and flaB genes of the first and the flaB gene of the second, was used. For the detection of $N$. mikurensis we used a duplex qPCR targeting the groEL gene of $N$. mikurensis and the msp2 gene of Anaplasma phagocytophilum. The sequences of primers and probes are given in Supplementary Table 1.

qPCRs were performed using the iQ Multiplex Powermix PCR reagent kit (Bio-Rad Laboratories, Hercules, USA), in a LightCycler 480 Real-Time PCR System (F. Hoffmann-La Roche, Basel, Switzerland). The triplex reaction mix for Borrelia 
species consisted of iQ multiplex Powermix, $100 \mathrm{nM}$ of the B-FlaB-Rc and B-FlaBRt primers, $200 \mathrm{nM}$ of the B-FlaB-F, FlabBm.motoiF2, and FlabB.m.motoiR3 primers, $400 \mathrm{nM}$ of the B-OspA_modF and B-OspA_borAS primers, $100 \mathrm{nM}$ of the B-OspAmodPatto probe, $200 \mathrm{nM}$ of the B-FlaB-Patto and FlabBm.motoiPro probes, and $3 \mu \mathrm{l}$ of template DNA in a final volume of $20 \mu \mathrm{l}$.

The duplex reaction mix for $A$. phagocytophilum and $N$. mikurensis consisted of iQ multiplex Powermix, $400 \mathrm{nM}$ of the primers, $200 \mathrm{nM}$ of the ApMSP2P probe, $400 \mathrm{nM}$ of the GroEL-P2a probe, and $3 \mu$ of template DNA in a final volume of $20 \mathrm{ul}$. Cycling conditions for both multiplex qPCR reactions included an initial activation of the iTaq DNA polymerase at $95^{\circ} \mathrm{C}$ for $5 \mathrm{~min}$, followed by 60 cycles of a $5 \mathrm{~s}$ denaturation at $95^{\circ} \mathrm{C}$, followed by a $35 \mathrm{~s}$ annealingextension step at $60^{\circ} \mathrm{C}$ (ramp rate $2.2^{\circ} \mathrm{C} / \mathrm{s}$ and a single point measurement at 60 ${ }^{\circ} \mathrm{C}$ ) and a cooling cycle of $37^{\circ} \mathrm{C}$ for $20 \mathrm{~s}$.

Analysis was performed using the second derivative calculations for crossing point values. For each run positive and negative controls and blank samples were included.

\section{Conventional PCR}

We targeted three genes located on different genetic elements of $B$. afzelii. i) 5S-23S intergenic spacer region (IGS) located on the chromosome, ii) $\mathrm{dbpA}$ located on plasmid Ip54, and iii) ospC located on plasmid cp26.

In order to build a phylogeny of the spirochetes found in the rodent ticks, we used the 5S-23S rDNA intergenic spacer (IGS). The PCR was performed according to the protocol described in Coipan et al. (Coipan et al. 2013b).

Conventional PCR for ospC was performed using the forward primer (OC6) described by Qiu et al. (Qiu et al. 2002) and a modification of the reverse primer (OC602) described by the same author, in a final concentration per reaction of $0.4 \mu \mathrm{M}$. The sequences of the primers are given in Supplementary Table 2 The PCR reaction was done using the HotStar Master Mix Kit (Qiagen, Venlo), with the following conditions: $15 \mathrm{~min} 94{ }^{\circ} \mathrm{C}$, then cycles of $20 \mathrm{~s} 94^{\circ} \mathrm{C}, 30 \mathrm{~s} 70{ }^{\circ} \mathrm{C}, 30 \mathrm{~s}$ $72{ }^{\circ} \mathrm{C}$ lowering the annealing temperature $1{ }^{\circ} \mathrm{C}$ each cycle until reaching $60^{\circ} \mathrm{C}$, then 40 cycles at this annealing temperature and ending by $7 \mathrm{~min} 72^{\circ} \mathrm{C}$.

Due to the high sequence variability of $\mathrm{dbpA}$, the conventional PCR for this gene was performed using three primer pairs, described in Supplementary Table 2. The PCR reaction mix and conditions were the same as for ospC. 
PCR products were sequenced using an ABI PRISM BigDye Terminator Cycle sequencing Ready Reaction kit (Perkin Elmer, Applied Biosystems). Sequences were confirmed by sequencing both strands (Sanger et al. 1977).

Storage, trimming and cleaning of the sequences were performed in BioNumerics version 7.0 (Applied Math, Belgium).

\section{Genetic analysis}

Sequences were aligned by MAFFT v 7.222 (Katoh and Standley 2013). Phylogenetic trees of all three genes were constructed by using a maximum likelihood algorithm, in RAxML, ran under RDP 4.56 (Martin and Rybicki 2000), with 100 bootstraps. Based on the clustering in the phylogenetic trees each allele of the three genes received a unique ID number/name. The ospC alleles were named according to Durand et al. (Durand et al. 2015). To identify similarities with sequences from other areas of Europe we downloaded IGS, $\mathrm{dbpA}$ and ospC sequences available in GenBank). The phylogenetic tree of IGS was rooted with $B$. garinii. For data visualization we constructed a phylogenetic tree of IGS, linked with a heatmap of allele types of the three genes, using the functions implemented in the ggtree package in R3.2.3 R Core (R_Core_Team 2015).

We tested for genetic differentiation between the various sites and rodent species using the AMOVA test implemented in Arlequin 3.5 (Excoffier and Lischer 2010), where we defined the rodent species as a group and the various sampling locations as populations.

\section{Statistical analysis}

Statistical analyses were performed in RStudio 0.99.891 running $\mathrm{R}$ 3.2.3 (R_Core_Team 2015). We calculated the fraction of infected larvae feeding on individuals of a vertebrate species for each of the pathogens: B. afzelii, B. miyamotoi, N. mikurensis, and A. phagocytophilum.

We tested the differences in infection prevalence of the two main rodent species for B. afzelii and for N. mikurensis, respectively, using Fisher's exact test. Subsequently, we tested whether the differential infection prevalence is due to the tick burden of the rodents or to the life history traits of the rodents (species, sex, weight). We checked for collinearity of the various parameters included in the models by calculating the variance inflation factor for each of them (package car). Variance inflation factors of most parameters were below 2, indicating absence of collinearity. The parameters that showed collinearity were the number of larvae and nymphs on the small mammals as well as the weight of the 
small mammals with the species. Consequently, we used only one of the two in our models for the prevalence of infection: we used species because we assume that the differences between genera are more prominent than those between members of different weights belonging to the same species. For the tick burden, we ran the models alternatively with larvae and nymphs.

We modelled the prevalence of infection as a function of the larval and nymphal burden, species, and sex of the rodent, using a generalized linear model (package stats) with a binomial distribution and logit link:

$y \sim \operatorname{Binomial}(n, p)$

$\operatorname{logit}(p)=$ intercept + species + stage + sex

where $y$ is the number of infected ticks, $n$ is the total number of ticks, $p$ is the prevalence.

We retained the model with the lowest Akaike Information Criterion (AIC) using a backward step approach.

To test the hypothesis that the rodents that have higher nymphal burden will also have a higher larval burden, and therefore a higher probability of transmission of the pathogenic bacteria from nymphs to larvae, we tested for the correlation between these two burdens using a generalized linear model with a negative-binomial distribution (due to observed overdispersion of the ticks on the rodents) and a log link (package MASS). We used the same parameters in the model as for the prevalence of infection and we selected the model with the lowest AIC using a backward step approach.

$\sim \operatorname{NegativeBinomial}(\lambda, \theta)$

$\log (\lambda)=$ intercept + species + stage + sex

To assess whether larvae infest equally on each rodent species or one species is more heavily parasitized than the other, we fitted the negative binomial distribution to the larval counts of each rodent species, based on the maximum likelihood method. The difference in the parameters of the negative binomial distribution between two rodent species was assessed for a statistical significance by the likelihood ratio test with two degrees of freedom.

\section{Acknowledgements}

We give thanks to Willem Takken and Maarten Schipper for fruitful discussions on the analysis and the results of our study. This study was financially supported by the Ministry of Health, Welfare and Sport (VWS). 


\section{Supplementary material}

Table S1. Primers and probes used in the qPCR analyses

\begin{tabular}{|c|c|c|}
\hline Target gene/primer \& probe & $\begin{array}{l}\text { Amplico } \\
\mathrm{n} \text { length }\end{array}$ & Sequence \\
\hline $\begin{array}{l}\text { ospA (outer membrane Protein } \\
\text { A, B. burgdorferi s.I.) }\end{array}$ & $\pm 139 \mathrm{bp}$ & \\
\hline B-OspA_modF & & 5'-AATATTTATTGGGAATAGGTCTAA-3' \\
\hline B-OspA_borAS & & 5'-CTTTGTCTTTTTCTTTRCTTACA-3' \\
\hline B-OspAmodPatto & & $\begin{array}{l}\text { 5'-Atto520- } \\
\text { AAGCAAAATGTTAGCAGCCTTGA-BHQ1-3' }\end{array}$ \\
\hline $\begin{array}{l}\text { flaB (flagelin B, B. burgdorferi } \\
\text { s.l.) }\end{array}$ & $\pm 89 \mathrm{bp}$ & \\
\hline B-FlaB-F & & $\begin{array}{l}\text { 5'- } \\
\text { CAGAIAGAGGTTCTATACAIATTGAIATAGA-3' }\end{array}$ \\
\hline B-FlaB-Rc & & 5'-GTGCATTTGGTTAIATTGCGC-3' \\
\hline B-FlaB-Rt & & 5'-GTGCATTTGGTTAIATTGTGC-3' \\
\hline B-FlaB-Patto & & $\begin{array}{l}\text { 5'-Atto425- } \\
\text { CAACTIACAGAIGAAAX'TAAIAGAATTGCTGAI } \\
\text { CA-Pho-3' }\end{array}$ \\
\hline $\begin{array}{l}\text { flaB miamotoi (flagelin } \mathrm{B}, B \text {. } \\
\text { miyamotoi) }\end{array}$ & $\pm 155 b p$ & \\
\hline FlabBm.motoiF2 & & 5'-AGAAGGTGCTCAAGCAG-3' \\
\hline FlabB.m.motoiR3 & & 5'-TCGATCTTTTGAAAGTGACATAT-3' \\
\hline FlabBm.motoiPro & & $\begin{array}{l}\text { 5'-ATTO647N- } \\
\text { AGCACAACAGGAGGGAGTTCAAGC-BHQ2-3' }\end{array}$ \\
\hline $\begin{array}{l}\text { msp2 (major surface protein } 2, \\
\text { A. phagocytophilum) }\end{array}$ & $\pm 77 b p$ & \\
\hline ApMSP2F & & 5'-ATGGAAGGTAGTGTTGGTTATGGTATT-3' \\
\hline ApMSP2R & & 5'-TTGGTCTTGAAGCGCTCGTA-3' \\
\hline ApMSP2P & & $\begin{array}{l}\text { 5'-FAM530- } \\
\text { TGGTGCCAGGGTTGAGCTTGAGATTG-BHQ1- } \\
\text { 3' }\end{array}$ \\
\hline $\begin{array}{l}\text { groEl (GroEl heatshock protein, } \\
\text { N. mikurensis) } \\
\text { GroEL-F2a }\end{array}$ & $\pm 102 b p$ & 5'-CCTTGAAAATATAGCAAGATCAGGTAG-3' \\
\hline GroEL-R2a & & 5'-CCACCACGTAACTTATTTAGCACTAAAG-3' \\
\hline GroEL-P2a & & $\begin{array}{l}\text { 5'-X"- } \\
\text { CCTCTACTAATTATTGCtGAAGATGTAGAAGG } \\
\text { TGAAGC-BHQ2-3' }\end{array}$ \\
\hline
\end{tabular}

$X^{\prime}$ = Black Hole Quencher 1-dT (BHQ 1-dT); X" = CAL Fluor Red 590 nm 
Table S2. Primers for conventional PCR and sequencing

\begin{tabular}{|c|c|c|}
\hline Target gene/primer & $\begin{array}{l}\text { Amplico } \\
\mathrm{n} \text { length }\end{array}$ & Sequence \\
\hline $\begin{array}{l}\text { OspC (Outer membrane Protein } \\
\text { C, B. afzelii) }\end{array}$ & $\pm 139 \mathrm{bp}$ & \\
\hline ospCF & & 5'-AAAGAATACATTAAGTGCGATATT-3' \\
\hline ospCRaf2 & & 5'-GGACTTGTAAGTTCTTTAACTG-3' \\
\hline $\begin{array}{l}\text { dbpA (decorin binding protein } \mathrm{A}, \\
\text { B. afzelii) }\end{array}$ & $\pm 89 \mathrm{bp}$ & \\
\hline dbpA-afF & & $\begin{array}{l}\text { 5'- } \\
\text { AAAATTATACTTACACTAACTTTACTTGCT-3' }\end{array}$ \\
\hline dbpA-Fafz1 & & 5'-TTGCTAGCCTGTTAGCAGCA-3' \\
\hline dbpA-Fafz2 & & 5'-TGGAATCATCAGTTAAAGACATTACAA-3' \\
\hline $\mathrm{dbpA}-\mathrm{afR2}$ & & 5'-TGTTTTCCACTTTTGCTTTCAGG-3' \\
\hline dbpA-Rafz3 & & 5'-TTTTGAGGGCTTCTTCATTTTTT-3' \\
\hline $\begin{array}{l}\text { IGS (5S-23S rDNA intergenic } \\
\text { spacer, } B \text {. burgdorferi s.I.) }\end{array}$ & $\pm 155 b p$ & \\
\hline IGS-F & & 5'-AGA AGG TGC TCA AGC AG-3' \\
\hline IGS-R & & 5'-TCG ATC TTT GAA AGT GAC ATA T-3' \\
\hline
\end{tabular}


Table S3. Infestation level of small mammals with I. ricinus ticks - centrality and dispersion descriptors; SD = standard deviation.

\begin{tabular}{|c|c|c|c|c|}
\hline Species & Descriptor & Larvae & Nymphs & All stages \\
\hline \multirow{5}{*}{$\begin{array}{l}\text { Apodemus } \\
\text { sylvaticus }\end{array}$} & Sum & 2489 & 46 & 2535 \\
\hline & Median & 25 & 0 & 26 \\
\hline & Range & $1-226$ & $0-7$ & $1-228$ \\
\hline & Mean & 50.8 & 0.9 & 51.7 \\
\hline & SD & 54.8 & 1.5 & 55.7 \\
\hline \multirow{5}{*}{ Myodes glareolus } & Sum & 534 & 20 & 554 \\
\hline & Median & 10 & 0 & 10 \\
\hline & Range & $2-125$ & $0-15$ & $2-140$ \\
\hline & Mean & 29.7 & 1.1 & 30.8 \\
\hline & SD & 37.1 & 3.5 & 39.6 \\
\hline \multirow{5}{*}{ Microtus agrestis } & Sum & 456 & 20 & 476 \\
\hline & Median & 30 & 2 & 37 \\
\hline & Range & $2-386$ & $1-9$ & $3-393$ \\
\hline & Mean & 91.2 & 4 & 95.2 \\
\hline & SD & 165.5 & 3.7 & 167.4 \\
\hline \multirow{5}{*}{ Sorex araneus } & Sum & 106 & 3 & 109 \\
\hline & Median & 2 & 0 & 2 \\
\hline & Range & $0-28$ & $0-1$ & $0-29$ \\
\hline & Mean & 5.0 & 0.1 & 5.2 \\
\hline & SD & 6.6 & 0.4 & 6.8 \\
\hline \multirow{5}{*}{ Sorex minutus } & Sum & 5 & 0 & 5 \\
\hline & Median & 2.5 & 0 & 2.5 \\
\hline & Range & $2-3$ & $0-0$ & $2-3$ \\
\hline & Mean & 2.5 & 0 & 2.5 \\
\hline & SD & 0.7 & 0.0 & 0.7 \\
\hline
\end{tabular}


Table S4. Observed and expected coinfections. Fisher's exact test was used to calculate the associations of pathogens. Significant positive associations $(p<$ 0.05 ) are shown in bold.

\begin{tabular}{l|r|r|r}
\hline Observed (\%) & B. miyamotoi & N. mikurensis & A. phagocytophilum \\
\hline B. afzelii & 0.00627 & 0.090909 & $7.84 \times 10^{-4}$ \\
B. miyamotoi & & 0.007837 & $7.84 \times 10^{-4}$ \\
N. mikurensis & & & $7.84 \times 10^{-4}$ \\
\hline Expected (\%) & B. miyamotoi & N. mikurensis & A. phagocytophilum \\
\hline B. afzelii & 0.003482424 & 0.043078883 & $3.87 \times 10^{-4}$ \\
B. miyamotoi & & 0.005538712 & $4.97 \times 10^{-5}$ \\
N. mikurensis & & & $6.15 \times 10^{-4}$ \\
\hline Fisher's exact-test (p-value) & B. miyamotoi & N. mikurensis & A. phagocytophilum \\
\hline B. afzelii & 0.069 & $<2.2 \times 10^{-16}$ & 0.4172 \\
B. miyamotoi & & 0.1909 & 0.06219 \\
N. mikurensis & & & 1 \\
\hline
\end{tabular}


Chapter 4

Table S5. Distribution of various haplotypes over the sampled rodents

IGS

\begin{tabular}{lrr}
\hline Species/haplotype & No. of rodents & No. of larvae \\
\hline A. sylvaticus & 24 & 67 \\
1 & 5 & 24 \\
2 & 5 & 20 \\
3 & 3 & 5 \\
4 & 2 & 6 \\
5 & 4 & 4 \\
6 & 2 & 3 \\
7 & 3 & 5 \\
Mi. agrestis & 3 & 5 \\
2 & 1 & 3 \\
6 & 1 & 1 \\
7 & 1 & 1 \\
\hline M. glareolus & 16 & 59 \\
1 & 3 & 16 \\
2 & 5 & 17 \\
3 & 1 & 7 \\
4 & 1 & 5 \\
5 & 3 & 4 \\
7 & 2 & 8 \\
$1 / 2$ & 1 & 2 \\
\hline Total & 43 & 131 \\
\hline
\end{tabular}

dbpA

\begin{tabular}{lrr}
\hline Species/haplotype & No. of rodents & No. of larvae \\
\hline A. sylvaticus & 24 & 67 \\
2 & 3 & 9 \\
3 & 1 & 16 \\
4 & 1 & 2 \\
5 & 3 & 5 \\
6 & 5 & 10 \\
7 & 4 & 5 \\
9 & 7 & 20 \\
\hline Mi. agrestis & & \\
1 & 3 & 6 \\
5 & 1 & 3 \\
6 & 1 & 2 \\
\hline
\end{tabular}




\begin{tabular}{lrr}
\hline Species/haplotype & No. of rodents & No. of larvae \\
\hline M. glareolus & 16 & 71 \\
1 & 3 & 15 \\
2 & 3 & 9 \\
4 & 1 & 6 \\
5 & 2 & 6 \\
6 & 2 & 7 \\
7 & 3 & 21 \\
8 & 1 & 6 \\
9 & 1 & 1 \\
\hline Total & 43 & 144 \\
\hline
\end{tabular}

ospC

\begin{tabular}{lrrr}
\hline Species/haplotype & $\begin{array}{r}\text { Haplotype } \\
\text { Durand et al., 2015 }\end{array}$ & No. of rodents & No. of larvae \\
\hline A. sylvaticus & A3 & 22 & 65 \\
1 & A9 & 6 & 29 \\
2 & A1 & 2 & 5 \\
3 & A7 & 2 & 3 \\
4 & & 4 & 6 \\
5 & A10 & 4 & 13 \\
6 & & 4 & 9 \\
\hline Mi. agrestis & A9 & 3 & 5 \\
2 & & 1 & 2 \\
5 & A10 & 1 & 2 \\
6 & & 1 & 1 \\
\hline M. glareolus & A3 & 16 & 64 \\
1 & A9 & 3 & 26 \\
2 & A1 & 3 & 10 \\
3 & A7 & 1 & 3 \\
4 & & 2 & 2 \\
5 & A10 & 4 & 15 \\
6 & A4 & 2 & 5 \\
7 & & 1 & 3 \\
\hline Total & & 41 & 134 \\
\hline
\end{tabular}


Chapter 4 


\section{Chapter 5}

\section{Spatiotemporal dynamics of emerging}

pathogens in questing Ixodes ricinus

This chapter has been published as:

Coipan, E.C., Jahfari, S., Fonville, M., Maassen, C.B., van der Giessen, J., Takken, W., Takumi, K., Sprong, H., 2013. Spatiotemporal dynamics of emerging pathogens in questing Ixodes ricinus. Frontiers in cellular and infection microbiology $3,36$. 


\section{Abstract}

Ixodes ricinus transmits Borrelia burgdorferi sensu lato, the etiological agent of Lyme disease. Previous studies have also detected Rickettsia helvetica, Anaplasma phagocytophilum, Neoehrlichia mikurensis and several Babesia species in questing ticks in The Netherlands.

In this study, we assessed the acarological risk of exposure to several tickborne pathogens (TBPs), in The Netherlands.

Questing ticks were collected monthly between 2006 and 2010 at 21 sites and between 2000 and 2009 at one other site. Nymphs and adults were analysed individually for the presence of TBPs using an array-approach. Collated data of this and previous studies were used to generate, for each pathogen, a presence/absence map and to further analyse their spatiotemporal variation.

Rickettsia helvetica (31.1\%) and B. burgdorferi sensu lato (11.8\%) had the highest overall prevalence and were detected in all areas. Neoehrlichia mikurensis (5.6 \%), A. phagocytophilum (0.8 \%), and Babesia spp. (1.7 \%) were detected in most, but not all areas. The prevalences of pathogens varied among the study areas from 0 to $64 \%$, while the density of questing ticks varied from 1 to $179 / 100 \mathrm{~m}^{2}$. Overall, $37 \%$ of the ticks were infected with at least one pathogen and $6.3 \%$ with more than one pathogen. One-third of the Borreliapositive ticks were infected with at least one other pathogen. Coinfection of $B$. afzelii with N. mikurensis and with Babesia spp. occurred significantly more often than single infections, indicating the existence of mutual reservoir hosts. Alternatively, coinfection of $R$. helvetica with either B. afzelii or N. mikurensis occurred significantly less frequent.

The diversity of TBPs detected in I. ricinus in this study and the frequency of their coinfections with $B$. burgdorferi s.l., underline the need to consider them when evaluating the risks of infection and subsequently the risk of disease following a tick bite. 


\section{Introduction}

In The Netherlands, the hard tick Ixodes ricinus is the main vector of a variety of human pathogens. The most prevalent tick-borne disease is Lyme borreliosis (Stanek et al. 2012). This multi-systemic disorder is caused by several members of the Borrelia burgdorferi sensu lato complex. Of the 18 genospecies of this complex (Margos et al. 2011), B. afzelii, B. garinii, B. spielmanii, B. bavariensis, and $B$. burgdorferi sensu stricto have already been detected in The Netherlands, in both patients and questing ticks. B. lusitaniae, and B. valaisiana were detected in questing $I$. ricinus, but their public health significance is less clear (Coipan et al. 2013a,Collares-Pereira et al. 2004, de Carvalho et al. 2008,Diza et al. 2004).

Over the last decades, the incidence of Lyme borreliosis has increased significantly in Europe (Smith and Takkinen 2006). A long-term retrospective study among general practitioners in The Netherlands has shown a continuing increase in consultations for tick bites and erythema migrans in the last decade (Hofhuis et al. 2006). The incidence of erythema migrans patients increased from 39 per 100,000 inhabitants in 1994 to 134 per 100,000 inhabitants in 2009.

Previous studies in The Netherlands have identified the presence of other pathogens in questing $I$. ricinus as well. Human babesiosis is caused by the intraerythrocytic protozoa Babesia divergens, B. venatorum (EU1), and B. microti (Vannier and Krause 2012). A recent study reported these three Babesia species in approximately $1 \%$ of questing I. ricinus (Wielinga et al. 2009). The spotted fever syndrome is caused by at least 15 different Rickettsia species, some of which are transmitted by I. ricinus (Heyman et al. 2010). Rickettsia conorii and $R$. monacensis are probably the most common tick-borne Rickettsiae to cause disease in Europe (Heyman et al. 2010), whereas the pathogenicity of $R$. helvetica is still questionable (Svendsen 2011). All three rickettsial species have been previously found in The Netherlands (Sprong et al. 2009) with local prevalences varying from $<1 \%$ ( $R$. conorii) to as high as $66 \%$ ( $R$. helvetica). Anaplasma phagocytophilum, the etiologic agent of human granulocytotropic anaplasmosis (Sprong et al. 2009), has been detected in Dutch ticks in several studies (Tijsse-Klasen et al. 2010,Tijsse-Klasen et al. 2011c). Neoehrlichia mikurensis can be considered an emerging zoonosis, as more than eight human cases have been described in Europe since 2010, while previously it was considered non-pathogenic. Despite an overall prevalence of $N$. mikurensis in questing ticks of approximately 7 \% (Jahfari et al. 2012), no human cases have been reported in The Netherlands. 


\section{Chapter 5}

Autochthonous tick-borne diseases other than Lyme disease have not been reported, except for a single case of granulocytotropic anaplasmosis in 1999 (van Dobbenburgh et al. 1999). This may be caused by lower pathogenicity, lack of overt symptoms, or lack of awareness of public and health professionals.

Multiple studies have reported coinfection with some of the tick-borne pathogens (TBPs) (Belongia 2002,Burri et al. 2011,Ginsberg 2008, Lommano et al. 2012,Nieto and Foley 2009, Reye et al. 2010). It is known that the severity of Lyme disease may be affected by simultaneous infections with other TBPs (Belongia 2002,Swanson et al. 2006). Some of them, such as $A$. phagocytophilum, modulate host immunity and increase susceptibility to various second pathogens, including B. burgdorferi (Holden et al. 2005, Thomas et al. 2001). Thus, coinfection might be partly responsible for the variability in clinical manifestations that are usually associated with Lyme borreliosis.

The acquirement of a tick-borne disease depends on many environmental, societal, and immunological factors, but it is always preceded by the bite of a tick infected with the causal agent. Previous studies have shown that the risk of infection of humans by TBPs depends mainly on the density of questing infected ticks - the acarological risk (Dister et al. 1997,Glass et al. 1994,Glass et al. 1995,Kitron and Kazmierczak 1997,Nicholson and Mather 1996). The study of mixed infections in questing ticks might, therefore, reveal patterns of coinfection of $B$. burgdorferi s.l. with two or more other pathogens, allowing us to generate hypotheses on the transmission cycle of some more obscure pathogens from the dynamics of better-known ones. The aim of this study was to assess the acarological risk of exposure to tick-borne pathogens in The Netherlands by comparing the abundances of questing ticks infected with B. burgdorferi s.l. and with other TBPs.

\section{Methods}

\section{Collection of ticks and tick data}

All ticks were collected by blanket dragging between 2006 and 2010 in 21 sites. In Duin\&Kruidberg field sampling was conducted between 2000 and 2009. The same sites were described in some previous studies regarding ticks and TBPs in The Netherlands (Gassner et al. 2011,Sprong et al. 2009,Tijsse-Klasen et al. 2011b,Wielinga et al. 2009,Wielinga et al. 2006). Based on morphological criteria, ticks were identified to species level, with stage and sex recorded. The density of ticks was estimated as the number of questing ticks per $100 \mathrm{~m}^{2}$. Additionally, data on the presence of ticks and TBPs in other 39 areas were collected from some previous studies that have used the same sampling and 
analysis methodology (Jahfari et al. 2012,Schouls et al. 1999,Tijsse-Klasen et al. 2010,Tijsse-Klasen et al. 2011c).

\section{DNA extraction of ticks}

All the collected ticks were immersed in $70 \%$ alcohol and stored at $-20{ }^{\circ} \mathrm{C}$ until the DNA extraction. DNA from questing ticks was extracted by alkaline lysis (Wielinga et al. 2006). Questing larvae were not taken into account as humans are generally bitten by either nymphs or adult I. ricinus (Hugli et al. 2009,TijsseKlasen et al. 2011c).

\section{PCR detection and identification of pathogens}

The presence of the DNA of different TBPs (Rickettsia spp., B. burgdorferi s.I., Ehrlichia/Anaplasma spp., and Babesia spp.) was determined by polymerase chain reaction (PCR) followed by reverse line blotting (RLB) as described before (Tijsse-Klasen et al. 2010,Wielinga et al. 2006). To minimize cross contamination and false-positive results, positive and negative controls were included in each batch tested by PCR and RLB assays. Furthermore, DNA extraction, PCR mix preparation, sample addition, and PCR analysis were performed in assigned separate labs. PCR products of some samples were sequenced by dideoxy-dye termination sequencing of both strands, and compared with sequences in GenBank (http://www.ncbi.nlm.nih.gov/), using BLAST (Altschul et al. 1990). The sequences were aligned and analysed using BioNumerics 6.6 (Applied Maths, Kortrijk, Belgium).

The prevalence of infection was calculated as the percentage of ticks infected with a certain microorganism.

\section{Acarological risk}

To calculate the densities of questing ticks infected with each of the five pathogens' genera, we multiplied the prevalence of infection with the density of questing ticks in each of the investigated sites.

\section{Correlation between prevalence and tick density}

For some pathogens, we noticed that the prevalence might correlate with the density of questing ticks at the sampling locations. To test this possibility we fitted a binomial model to our data, by defining the prevalence of infection as an exponential function of the tick density $(d)$ at each sampling location. Knowing that the number of infected ticks $(k)$ out of the total number of ticks tested $(n)$, is binomially distributed with a probability $(p)$, we used the function $p=$ $a \operatorname{Exp}[b d], 0<a<1$, to test an alternative model $(b<0)$ against a null model 
( $b=0$ ) by a likelihood-ratio test. The alternative (decreasing exponential) model fitted significantly better to our data with $p$-value $P \leq 0.05$.

\section{Seasonal dynamics}

To test for the seasonal dynamics of infection in ticks, a binomial model for the probability of infection $(p)$ was fitted to our data, in combination with the sampling days $(t)$ and pooled over the sampling locations and development stages. The probability of infection was a logit function $p=\frac{e^{y}}{1+e^{y}}$ and we modelled seasonality by using the trigonometric function $y=a+b \cos (2 \pi t /$ $365)+c \sin (2 \pi t / 365)$ to describe an oscillation with a period of one year and possible phase shift. For each pathogen, we tested the seasonal model against the non-seasonal model $(y=a)$ based on a likelihood ratio test. The seasonal model fitted significantly better to our data with $\mathrm{p}$-value $P \leq 0.05$. All the statistical analyses were performed using Wolfram Mathematica 9.

\section{Results}

The mean density of questing nymphs and adult ticks varied greatly between sites, from as low as 1 (at Houtvesterijen Heide) up to $179 / 100 \mathrm{~m}^{2}$ (at Duin\&Kruidberg; Table 2), results that are consistent with previous Dutch studies (Wielinga et al. 2006).

\section{Pathogen detection and identification}

A total of 5570 questing nymphs and adult $I$. ricinus from 22 different study areas were tested for the presence of TBPs by PCR amplification followed by RLB (Table 1). The recently identified $B$. bavariensis reacted consistently with our B. garinii probe (Margos et al. 2009), and therefore we grouped these two Borrelia genospecies. Five Borrelia genospecies were found in this study in all twenty-two study areas (Table 1), with the overall prevalence (11.8\%) inscribed in the interval of average European prevalence (Rauter and Hartung 2005), and comparable with previous studies in The Netherlands (Gassner et al. 2011,Wielinga et al. 2006). Borrelia afzelii was predominant (6.7 \%), followed by B. garinii/B. bavariensis (1.5\%), B. valaisiana (1.2\%), and B. burgdorferi sensu stricto $(0.2 \%)$. The remaining fraction of the Borrelia positive samples could not be further identified to the species level by RLB. Sequencing several of these samples revealed the presence of $B$. spielmanii, corroborating previous findings of this genospecies in The Netherlands (Wang et al. 1999a). Borrelia lusitaniae, which was recently found in The Netherlands (Tijsse-Klasen et al. 2010), was not detected in this study. 
Table 1. Presence of microorganisms in questing $I$. ricinus nymphs and adults. Tick lysates were subjected to PCR followed by Reverse Line Blotting. PCR products that specifically reacted to the generic ("catch all") probes, but that could not be further specified to the (geno)species level were designated as Untypeable. Reverse Line Blot analysis could not distinguish between $B$. garinii and the recently identified $B$. bavariensis. Calculations of prevalence were based on all tick lysates that were analysed (n).

\begin{tabular}{|c|c|c|c|}
\hline Pathogen & $\begin{array}{r}\text { Positive/ Tested } \\
\text { I. ricinus (n) }\end{array}$ & $\begin{array}{l}\text { Prevalence } \\
\text { I. ricinus (\%) }\end{array}$ & $\begin{array}{l}\text { Positive/Tested } \\
\text { Study areas (n) }\end{array}$ \\
\hline Borrelia burgdorferi sl. & $628(5308)$ & $11.8 \%$ & $22 / 22$ \\
\hline - Borrelia afzelii (\& B. ruski) & $355(5308)$ & $6.7 \%$ & $22 / 22$ \\
\hline $\begin{array}{l}\text { Borrelia garinii/ } B \text {. } \\
\text { bavariensis }\end{array}$ & $79(5308)$ & $1.5 \%$ & $17 / 22$ \\
\hline - Borrelia valaisiana & $64(5308)$ & $1.2 \%$ & $18 / 22$ \\
\hline - Borrelia burgdorferi ss. & $10(5308)$ & $0.2 \%$ & $8 / 22$ \\
\hline - Untypeable Borrelia & $133(5308)$ & $2.5 \%$ & $20 / 22$ \\
\hline Rickettsia helvetica & 1265 (4061) & $31.1 \%$ & $19 / 19$ \\
\hline Rickettsia conorii & $3(4061)$ & $0.1 \%$ & $1 / 19$ \\
\hline Untypeable Rickettsia & $33(4061)$ & $0.8 \%$ & $13 / 19$ \\
\hline Anaplasma phagocytophilum & $44(5343)$ & $0.8 \%$ & $14 / 22$ \\
\hline Neoehrlichia mikurensis & $300(5343)$ & $5.6 \%$ & $18 / 22$ \\
\hline Ehrlichia canis & $5(5343)$ & $0.1 \%$ & $4 / 22$ \\
\hline Untypeable Ehrlichia & $99(5343)$ & $1.9 \%$ & $16 / 22$ \\
\hline Babesia microtii & $17(4238)$ & $0.4 \%$ & $6 / 19$ \\
\hline Babesia venatorum (EU1) & $41(4238)$ & $1.0 \%$ & $14 / 19$ \\
\hline Babesia divergens & 1 (4238) & $0 \%$ & $1 / 19$ \\
\hline Untypeable Babesia & $12(4238)$ & $0.3 \%$ & $12 / 19$ \\
\hline
\end{tabular}

Rickettsia helvetica was most frequently detected in tick lysates, with a prevalence of $31 \%$ (Table 1). Thirty-three Rickettsia isolates could not be identified up to the species level by RLB. Sequencing of these samples revealed the presence of $R$. monacensis, which was reported in The Netherlands before (Sprong et al. 2009). Rickettsia conorii was detected in only three questing ticks from one study area (Veldhoven). Anaplasma phagocytophilum-infected ticks were recorded with an overall prevalence of only $0.8 \%$ (Table 1). Neoehrlichia mikurensis DNA was found with a global prevalence of $5.6 \%$ (Table 1). Ehrlichia canis DNA was detected in only 5 tick lysates from four different study areas, which resulted in an overall prevalence of $0.1 \%$ (5/5343). Ninety-nine Ehrlichia isolates could not be identified to the species level neither by RLB nor by sequencing. Babesia venatorum, formerly also known as B. EU1 (Duh et al. $2005)$, was present with a global prevalence of $1.0 \%$ (41/4238). The prevalence 


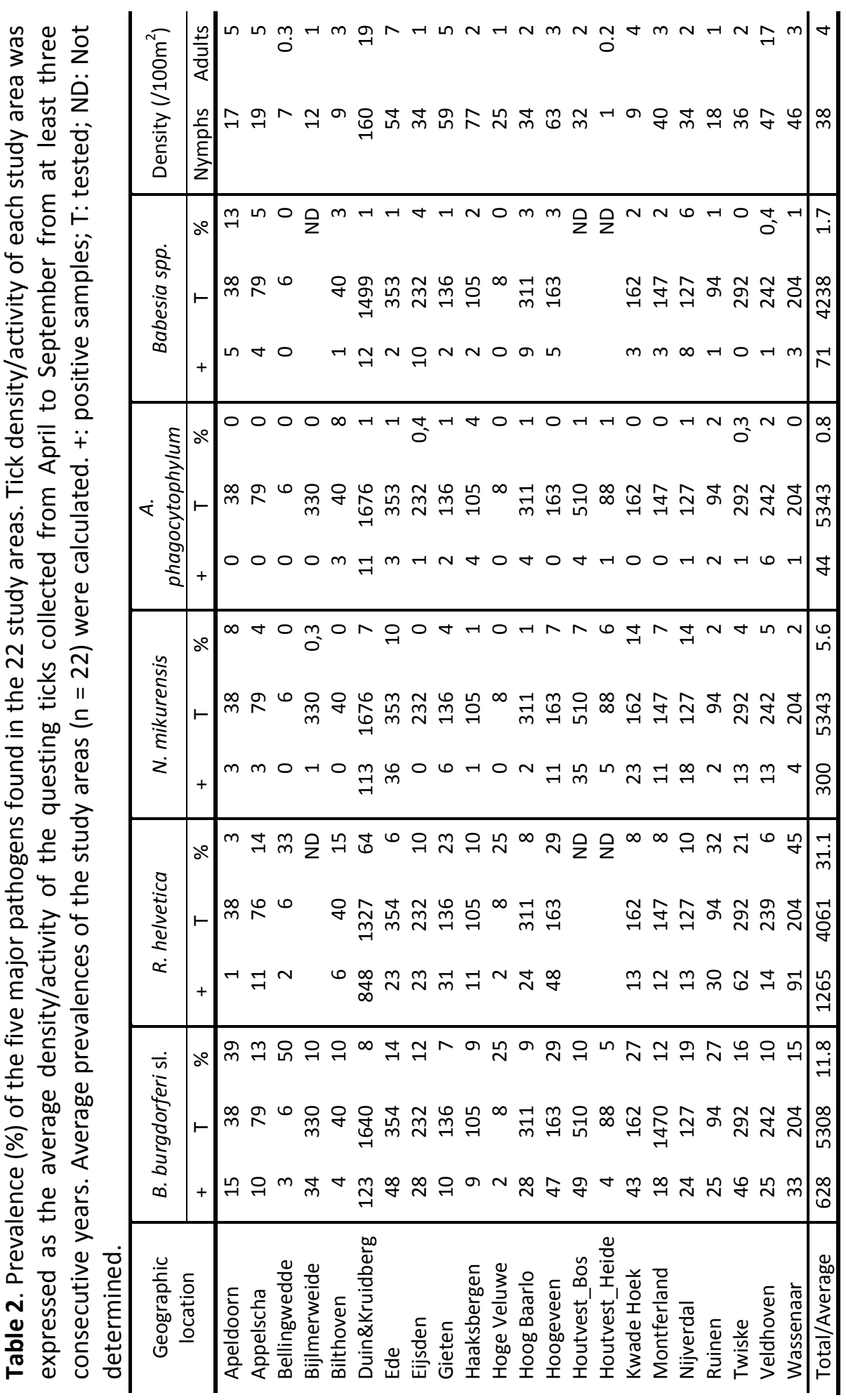


of $B$. microti in questing ticks was $0.4 \%$ (17/4238), and the protozoan was detected in 6 from 19 sites. Only one tick from the Duin\&Kruidberg area contained the DNA of previously reported B. divergens (Wielinga et al. 2009). Twelve Babesia sp. could not be further identified by neither RLB, or sequencing. The average prevalence of Babesia-positive ticks in the study areas was $1.6 \%$ (Table 1).

\section{Spatial distribution and variation}

Collated data were used to generate presence/absence maps of the five major TBPs in The Netherlands (Figure 1). The presence/absence of Borrelia spp., R. helvetica, A. phagocytophilum, N. mikurensis, and Babesia spp. was assessed for $61,24,39,39$, and 25 locations respectively. The presence of these pathogens was observed in 58, 24, 33, 20 and 18 areas, respectively, heterogeneously distributed across The Netherlands. The few absence points were scattered over The Netherlands as well, and did not cluster in any geographic region (Figure 1).

Borrelia prevalence was between $5 \%$ (Houtvesterijen Heide) and $50 \%$ (Bellingwedde; where only six ticks were tested), while for $R$. helvetica it varied even more, from $3 \%$ in some sites (Apeldoorn), to $64 \%$ in others (Duin\&Kruidberg) (Table 2). Lower variations in prevalences were observed for N. mikurensis, A. phagocytophilum and Babesia spp. (Table 2). For N. mikurensis, the prevalence was on average of $5 \%$, but some areas displayed values of over $10 \%$ (Table 2). Babesia spp. showed an overall prevalence of $1.7 \%$, similarly to Germany and Luxembourg (Hartelt et al. 2004, Reye et al. 2010). A. phagocytophilum was the least prevalent pathogen in our study, with a mean prevalence of $0.8 \%$ - comparable with the $0.5-1 \%$ prevalence found in different European countries (Hildebrandt et al. 2010,Koci et al. 2007,Severinsson et al. 2010). However, one of the sites displayed a tenfold higher prevalence than average (Bilthoven $8 \%$, Table 2).

Identification of high risk-areas depends on both pathogen prevalence and density of questing ticks (nymphs and adults). The density of questing ticks varied between $1 / 100 \mathrm{~m}^{2}$ (Houtvesterijen Heide) and 179/100 $\mathrm{m}^{2}$ (Duin\&Kruidberg; Table 2). The density of questing Borrelia-infected ticks varied between 0 and 19 ticks per $100 \mathrm{~m}^{2}$ (Figure 2), whereas the maximum densities of A. phagocytophilum, N. mikurensis and Babesia spp. infected ticks were 3.0, 13, and 2.9 ticks per $100 \mathrm{~m}^{2}$, respectively. The density of questing $R$. helveticainfected ticks varied between 0 and 22 ticks per $100 \mathrm{~m}^{2}$, with one notable exception: Duin\&Kruidberg area had both a high tick density and an 


\section{Chapter 5}

exceptionally high $R$. helvetica prevalence, which resulted in a density of questing $R$. helvetica-infected ticks of up to 119 ticks per $100 \mathrm{~m}^{2}$.

Based on a likelihood ratio test, performed for a decreasing model and a constant one, we detected a significant negative correlation between the density of questing ticks and the infection prevalence with $B$. burgdorferi s.l. ( $p=3.6 \mathrm{x}$ $\left.10^{-10}\right)$ and Babesia spp. $\left(p=4.9 \times 10^{-5}\right)$ (Figure 3). On the other hand, there was no correlation found between these variables for $R$. helvetica $(p=1.0), N$. mikurensis $(p=1.0)$ and A. phagocytophilum ( $p=0.69$ ) (Figure 3). Graphs for the density of infected questing ticks against the density of questing ticks revealed that the former is linearly increasing with the latter for $R$. helvetica, $N$. mikurensis and A. phagocytophilum (Figure 4). For the other two pathogens Babesia spp. and B. burgdorferi s.l., the density of infected questing ticks reached the maximum values at densities of questing ticks of 119 and 268 respectively (Figure 4).

\section{Temporal variation}

To gain insight into long-term dynamics of ticks and their pathogens, we analysed the data obtained from Duin\&Kruidberg, where a ten-year (2000 2009) tick-surveillance was performed. This area was selected at that time because of its unusual high tick density/activity. The prevalences of all pathogens were relatively stable over the past decade (B. burgdorferi s.I. 7.0\%, B. afzelii 4.6 $\%$, A. phagocytophilum $0.7 \%$, R. helvetica $65 \%$, Babesia spp. $1.1 \%$ ), except for N. mikurensis, whose prevalence increased from $3.5 \%(2000-2007)$ to $12 \%$ in the last two-years interval (2008-2009). The average density/activity of adult ticks remained relatively low with 7-34 ticks per $100 \mathrm{~m}^{2}$. The average density/activity of nymphal ticks was more pronounced (102-410 ticks per $\left.100 \mathrm{~m}^{2}\right)$ and peaked in 2004-2005 (Figure 5).

The likelihood ratio test detected similar decreasing trends in the temporal relation between the prevalence and the tick density as for the spatial variation analysis (not shown). Despite the inverse relationship between the prevalence and the tick density, the peaks of density/activity of infected ticks coincided with the peak of high densities of questing ticks in 2004-2005 (Figure $5)$.

\section{Coinfection}

Overall, $37 \%(2064 / 5570)$ of the ticks was infected with one or more pathogens and $6.3 \%(350 / 5570)$ with more than one pathogen of different genera. Furthermore, $37 \%(234 / 628)$ of the Borrelia-positive ticks were infected with at least one other pathogen of a different genus. Almost $5 \%(29 / 628)$ of the 

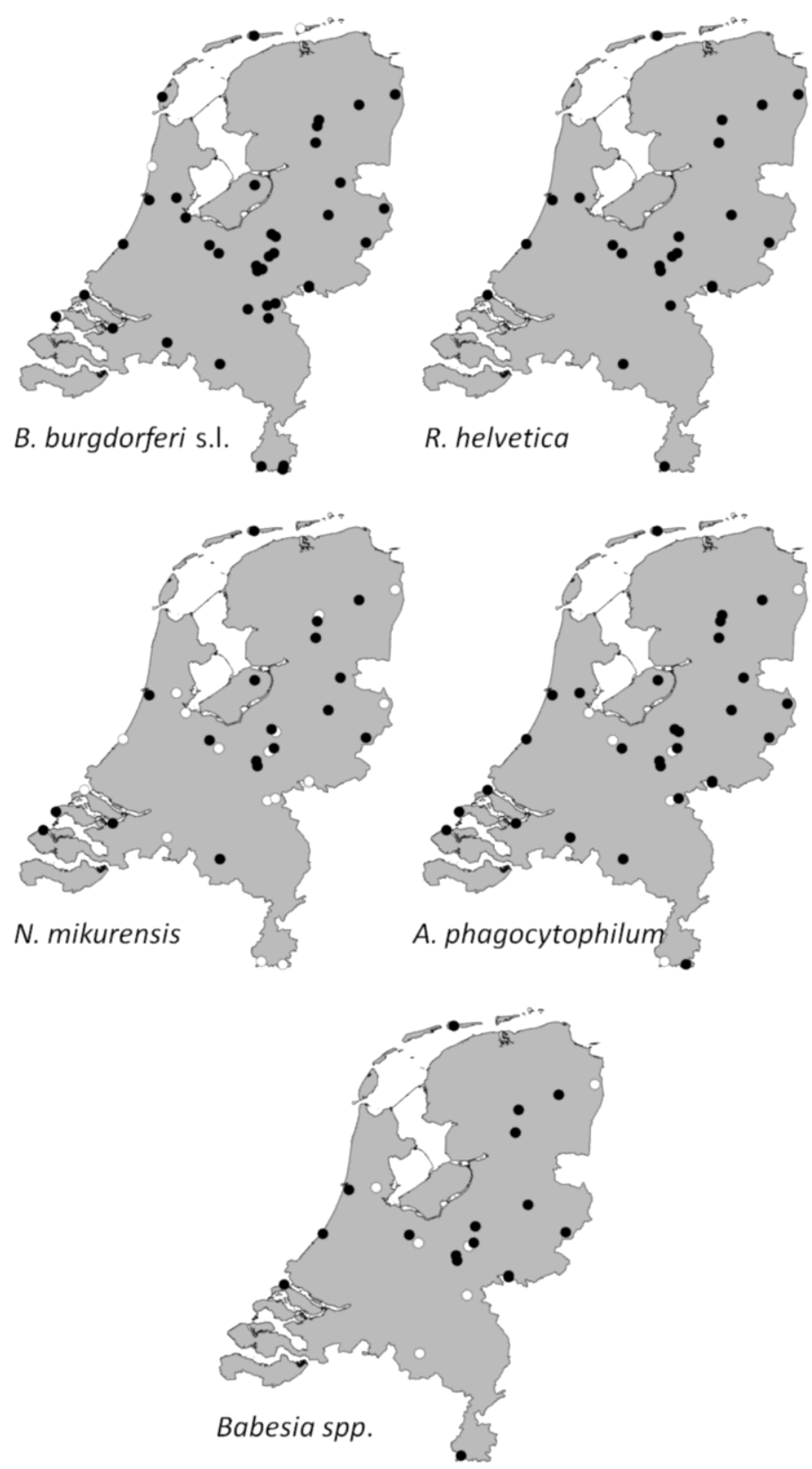

Figure 1. Aggregated presence/absence map of questing $I$. ricinus nymphs/adults infected with B. burgdorferi s.I. (A), R. helvetica (B), N. mikurensis (C), A. phagocytophilum (D), Babesia species (E). The black dots represent presence of the microorganism; the white ones represent absence. Presence/absence points from previous studies were also incorporated. 


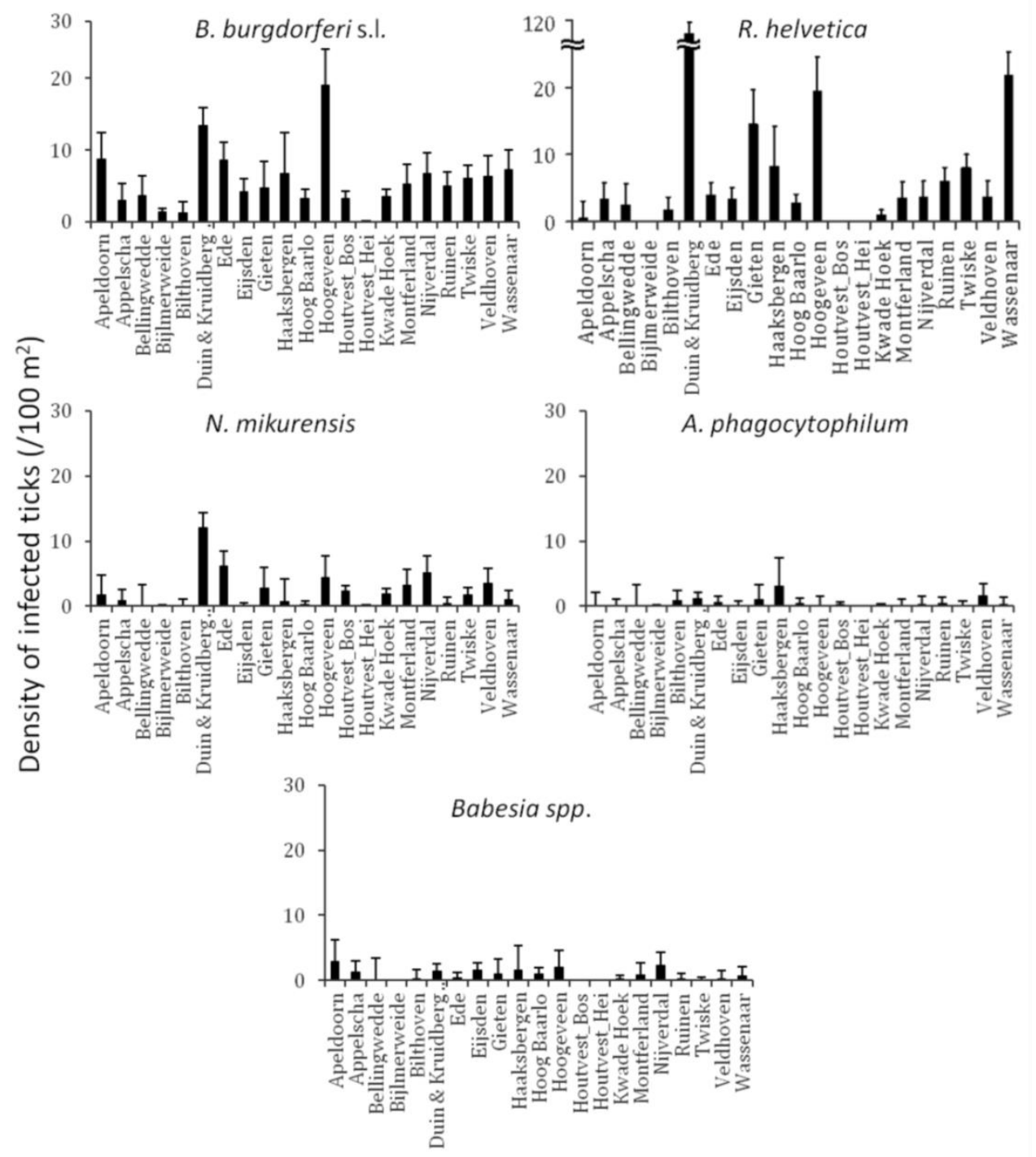

Figure 2. Identification of high risk-areas depends on both prevalence and tick density/activity. Their calculated product defines the density/activity of infected ticks (nymphs and adults $/ 100 \mathrm{~m}^{2}$ ). The error bars depict the upper limit of the $95 \%$ confidence interval. Duin\&Kruidberg's density of $R$. helvetica infected ticks reaches to $119 / 100 \mathrm{~m}^{2}$. 

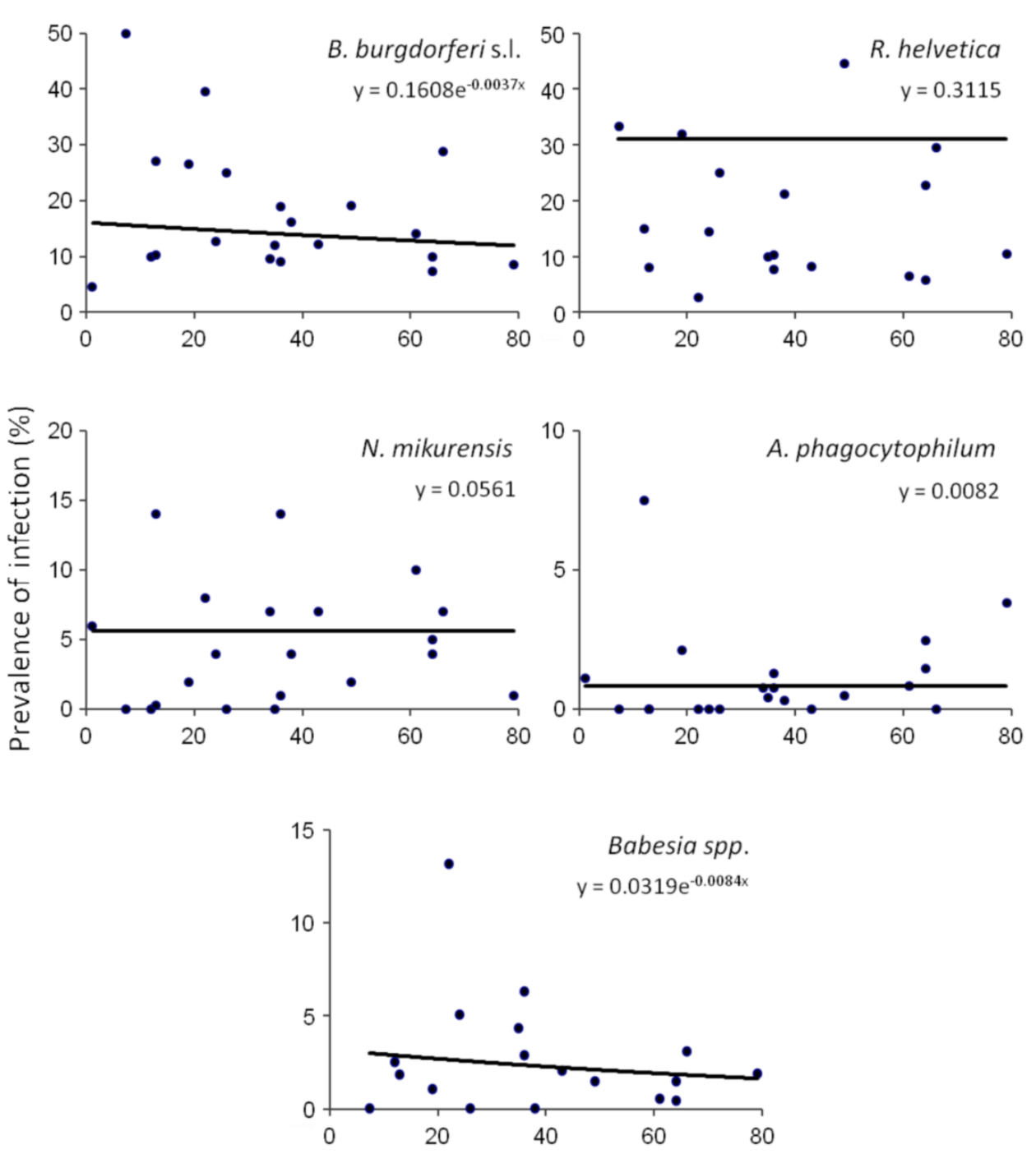

Density of questing ticks $\left(/ 100 \mathrm{~m}^{2}\right)$

Figure 3. Density and prevalence relations. Significant negative correlations between the density of questing ticks and the infection prevalence were found for B. burgdorferi s.I. $\left(p=3.6 \times 10^{-10}\right)$ and Babesia spp. $\left(p=4.9 \times 10^{-5}\right)$. On the other hand, there was no correlation found between these variables for R. helvetica $(p=1.0), N$. mikurensis $(p=1.0)$ and A. phagocytophilum $(p=0.69)$. Note that due to the very small exponents, the curves look approximately linear, although they are in fact exponential, as explained in the text. The data set included all of the areas except for Duin\&Kruidberg. 

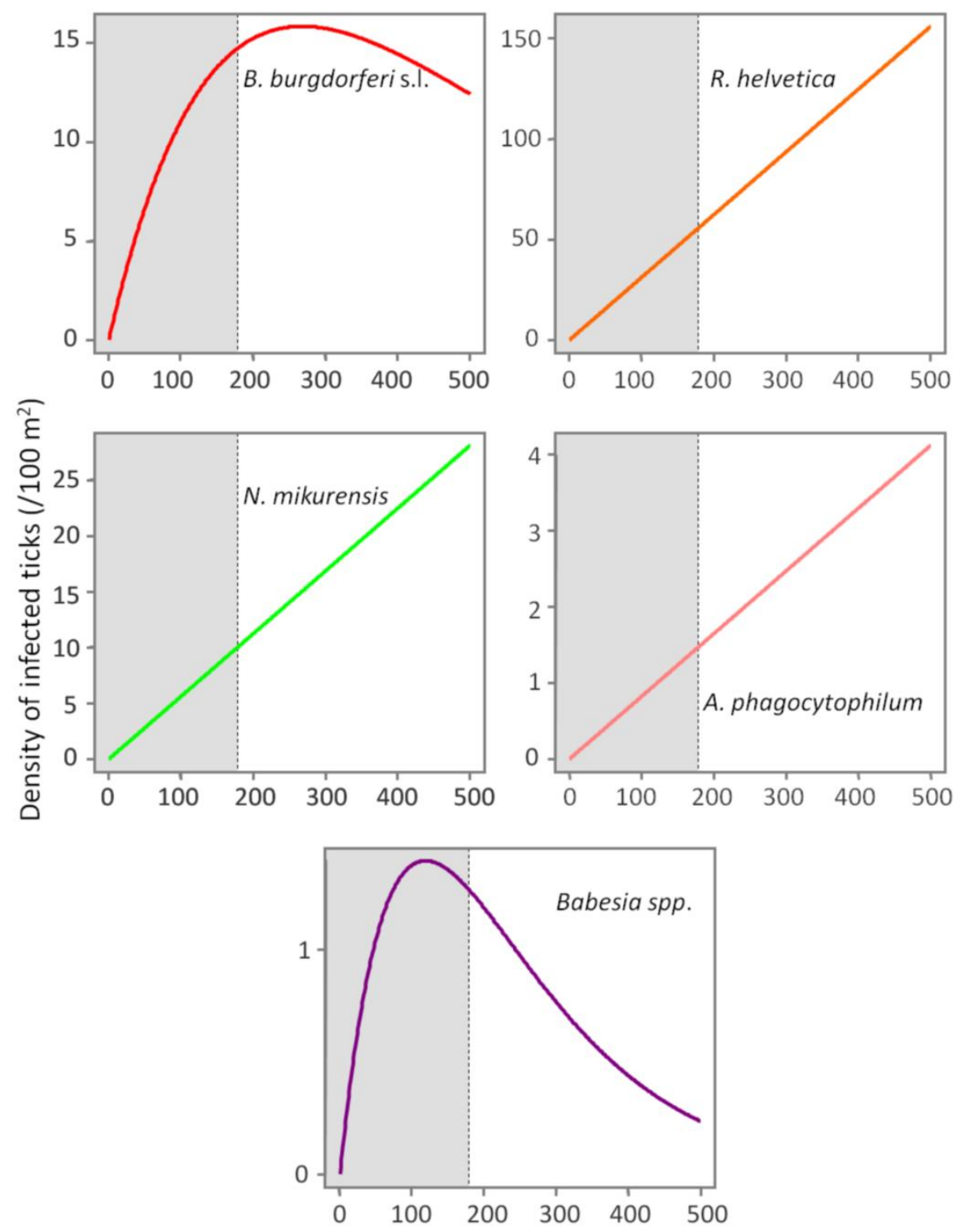

Density of questing ticks $\left(/ 100 \mathrm{~m}^{2}\right)$

Figure 4. Evolution of the density of infected ticks (y-axis) with the density of questing ticks ( $x$-axis). The density of infected ticks is obtained by fitting a model $(y=a \operatorname{Exp}[b d])$ to a range of questing ticks densities. The numbers are expressed as ticks $/ 100 \mathrm{~m}^{2}$. The grey area marks the normal questing ticks densities $\left(0-179 / 100 \mathrm{~m}^{2}\right)$ in The Netherlands. 
Borrelia-positive ticks were also positive for three or more other pathogens. One tick carried the DNA of B. afzelii, $R$. helvetica, N. mikurensis, and B. microti. Mixed infections, involving two or three Borrelia genospecies, occurred in only $0.3 \%$ (15/5308) of the tick lysates. Coinfection of B. afzelii with $N$. mikurensis or with Babesia spp. occurred significantly more than random, whereas infection of $R$. helvetica with either B. afzelii or $N$. mikurensis occurred significantly less frequent (Table 3).

\section{Seasonal dynamics}

Seasonality modelling of the prevalence indicated a different periodicity of the analysed pathogens (Figure 6). Thus, B. afzelii, N. mikurensis and Babesia spp. showed highest prevalences in ticks at time periods corresponding to October, while non-afzelii $B$. burgdorferi and $R$. helvetica had the highest prevalence around June. Annual prevalence of $A$. phagocytophilum was not seasonal.

\section{Discussion}

In order to assess the relative risk of acquiring a tick-borne infection in The Netherlands, the abundance of questing ticks infected with $B$. burgdorferi sl. and four other genera of TBPs were compared.

Our study revealed the nationwide circulation of TBPs in enzootic cycles. Although the most common tick-borne infection is acknowledged to be Lyme borreliosis, our results showed that there are other pathogens present in questing ticks at prevalences comparable with Borrelia burgdorferi (i.e. $R$. helvetica, Table 2). Rickettsia helvetica was the most frequently detected pathogen, its $31.1 \%$ average prevalence being among one of the highest in Europe (range 1.5 to more than 40.6 \% (Christova et al. 2003,Severinsson et al. 2010)). A previous study from our laboratory found $R$. helvetica not only in vertebrate hosts, but also in tick larvae at comparable prevalences as for the other tick stages, indicating a high efficiency of transovarial transmission (Sprong et al. 2009).

Due to the fact that our investigations only detected the DNA of the microorganisms under discussion, and not the viable cells, we cannot asseverate their infectiousness for other vertebrate hosts. However, previous studies implicate Ixodes ricinus ticks as vectors for these microorganisms (Ackermann et al. 1984,Barbour et al. 1983,Bonnet et al. 2007,Gray et al. 2002, Heyman et al. 2010,Jahfari et al. 2012,Kjemtrup and Conrad 2000,Lotric-Furlan et al. 1998,Parola and Raoult 2001,Rydkina et al. 1999,Sprong et al. 2009), and therefore the risk for public health should not be neglected. Although no human 

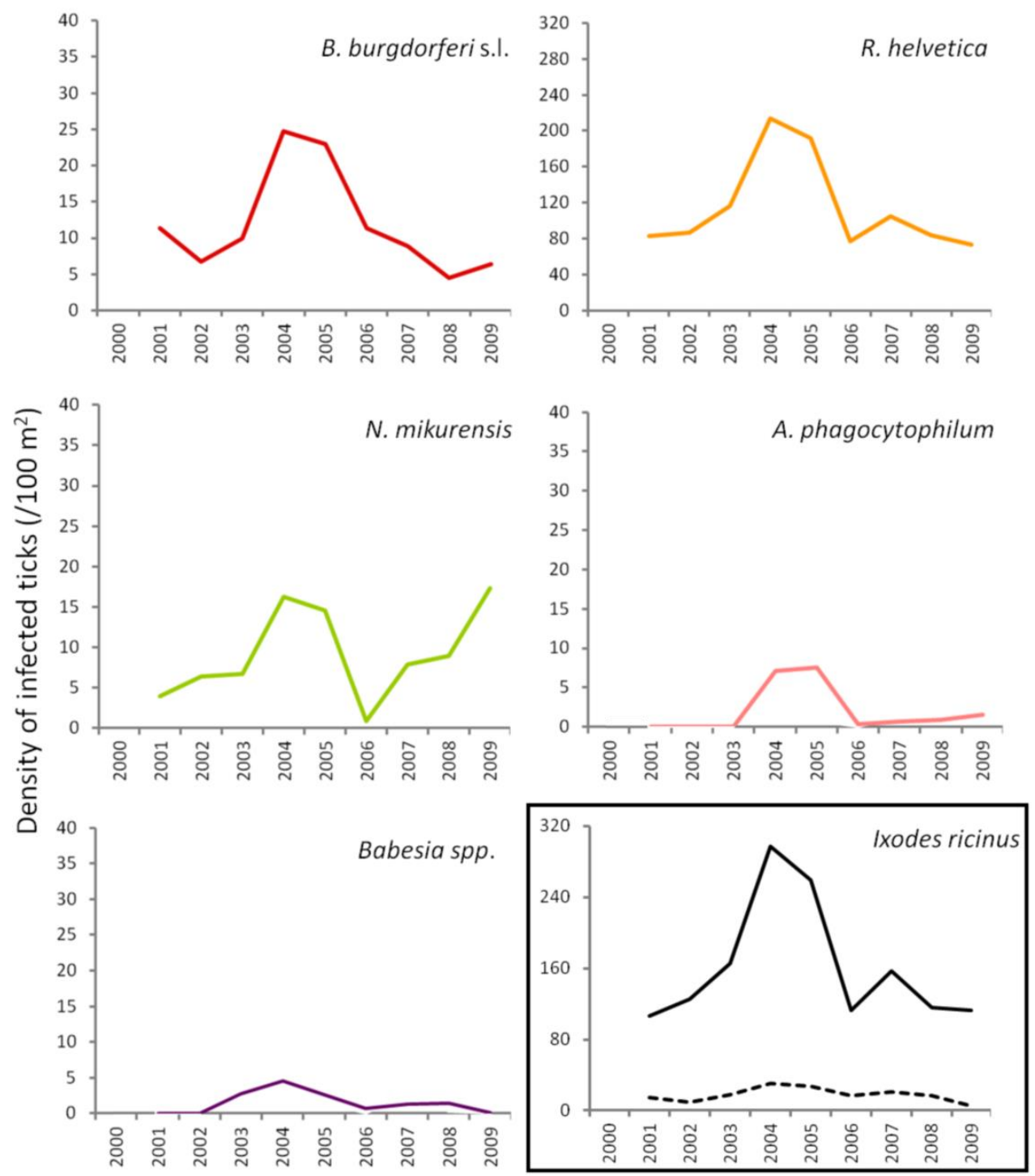

Figure 5. Changing average (2 years) of density of infected ticks and tick density/activity in Duin\&Kruidberg area. Density/activity of nymphs and adults are shown in the bottom right graph as continuous and dotted line, respectively. 
disease with the organisms other than B. burgdorferi sl. was reported so far in The Netherlands, it is known that infection with some of them (e.g. Ehrlichia) is generally either asymptomatic or mild, self-limiting diseases (Ismail et al. 2010).

\section{Spatial distribution and variation}

All the pathogens were observed in most of the areas in which investigations were conducted, regardless of the geographical position. The absence in certain areas might be explained by the relatively low number of ticks collected/tested (Table 2). The prevalences of infection in the ticks varied significantly between the areas investigated. The lack of a full perspective on the host community at each of the sites does not allow us to make a definite statement on why we see such a variation of the prevalence of infection. We propose, however, that the extremely high local variability of the pathogens may be associated with the differences in host assemblages in the investigated habitats. As ticks can feed on many different animals and every host species has a unique reservoir competence (e.g. rodents being the most competent reservoirs of $B$. afzelii (Gern and Humair 2002a)), the presence of different hosts in different communities affects the prevalence of infection with various microorganisms.

In terms of the risk for public health, neither the density of questing ticks, nor the prevalence of infection alone, has any significance. Instead, it is their product - the density of infected questing ticks - that defines high or low risk areas (Dister et al. 1997,Glass et al. 1994,Glass et al. 1995,Kitron and Kazmierczak 1997,Nicholson and Mather 1996). The densities of infected ticks were above $10-20 / 100 \mathrm{~m}^{2}$ for $B$. burgdorferi s.I. and $R$. helvetica in some sites, with as many as $117 R$. helvetica infected ticks per $100 \mathrm{~m}^{2}$ for Duin\&Kruidberg (Figure 2). In the case of $N$. mikurensis, A. phagocytophilum and Babesia spp. the densities of infected ticks were up to $10 / 100 \mathrm{~m}^{2}$ (Figure 2). Considering that these are calculated as average values for an entire season, it is therefore inevitable that the densities of infected questing ticks are actually higher for peak months of tick activity (i.e. May-June (Gassner et al. 2011)).

We noticed that in some areas, where tick densities were highest, the mean prevalence of Borrelia infection had very low values $(8 \%$ for Duin\&Kruidberg; Table2). Using a log-likelihood ratio statistics, we tested the hypothesis of a constant prevalence over the range of questing ticks density. The test confirmed the independence of the two variables but only for $R$. helvetica, $N$. mikurensis, and A. phagocytophilum, while for B. burgdorferi and Babesia spp. it indicated a slight negative correlation of the prevalence with the tick density (Figure 3). Thus, we would expect that the density of ticks infected with $B$. 


\section{Chapter 5}

burgdorferi and Babesia spp. would decrease as the density of questing ticks increases. Plotting the density of infected questing ticks as an exponential function of the questing ticks' densities, however, revealed that over the usual range of tick densities the density of infected ticks is also increasing, and the downward trend might be observed only for tick densities of over 100 (for Babesia spp.) or $200 / 100 \mathrm{~m}^{2}$ (for B. burgdorferi) (Figure 4). This observation is consistent with the finding made by Randolph (Randolph 2001) that, in Europe the density of Borrelia infected ticks depends much more on the density of all ticks than on the infection prevalence, and that only in areas where the tick density is unusually high $\left(100-450 / 100 \mathrm{~m}^{2}\right)$ is the infection prevalence consistently low.

Table 3. Observed and expected coinfections. Chi-square tests were used to calculate the associations of several combinations of pathogens *Significant positive associations and ${ }^{* *}$ significant negative associations $(p<0.05)$ are shown in bold.

\begin{tabular}{lrrrr}
\hline Observed (\%) & R. helvetica & A. phagocytophilum & N. mikurensis & Babesia spp. \\
\hline Borrelia (all) & 3.3 & 0.1 & 1.6 & 0.4 \\
B. afzelii & 1.8 & 0.0 & 1.3 & 0.3 \\
R. helvetica & & 0.3 & 2.2 & 0.5 \\
A. phagocytophilum & & & 0.0 & 0.0 \\
N. mikurensis & & & 0.1 \\
\hline Expected (\%) & R. helvetica & A. phagocytophilum & N. mikurensis & Babesia spp. \\
\hline Borrelia (all) & 3.9 & 0.1 & 0.7 & 0.2 \\
B. afzelii & 2.2 & 0.1 & 0.4 & 0.1 \\
R. helvetica & & 0.3 & 1.9 & 0.5 \\
A. phagocytophilum & & & 0.0 & 0.0 \\
N. mikurensis & & & & 0.1 \\
\hline Chi' ${ }^{2}$-test (p-value) & R. helvetica & A. phagocytophilum & N. mikurensis & Babesia spp. \\
\hline Borrelia (all) & $\mathbf{0 . 0 3 * *}$ & 0.30 & $\mathbf{0 . 0 0 *}$ & $\mathbf{0 . 0 1 *}$ \\
B. afzelii & $\mathbf{0 . 0 3 * *}$ & 0.24 & $\mathbf{0 . 0 0 ^ { * }}$ & $\mathbf{0 . 0 0 ^ { * }}$ \\
R. helvetica & & 0.80 & $\mathbf{0 . 0 5 * *}$ & 0.77 \\
A. phagocytophilum & & & 0.10 & 0.42 \\
N. mikurensis & & & & 0.66 \\
\hline
\end{tabular}

One drawback in the assessment of the density of questing infected ticks is that one of its components, namely the density of questing ticks, is reliant on many variable factors like microclimate, host composition, and vegetation (Gray et al. 1998). Moreover, there is not a fail-safe method for quantifying it, as weather and vegetation structure strongly impact on the efficiency of the most common method - tick flagging. Development of alternative quantification 
methods, insusceptible to the aforementioned factors, might lead to more accurate risk estimation for exposure to ticks and the pathogens they harbour.

\section{Temporal variation}

In terms of temporal variation, the longest series of data we had was for 10 successive years (Duin\&Kruidberg, Figure 5). At this site, the density of questing ticks was highest in 2004-2005, and it was due to a steep increase in the number of questing nymphs. The trend line indicated the maintenance of relatively constant prevalences for B. burgdorferi, $A$. phagocytophilum, and $R$. helvetica. Babesia prevalence showed a slight decrease over time while, on the contrary, N. mikurensis showed a steep increase (almost three-fold). The variations in both tick density and prevalences of various pathogens in ticks might indicate yearly fluctuations in the composition and availability of reservoir hosts. For example, a mast year might have been responsible for the increment in small mammals' population size (i.e. rodents), with the upsurge of the preimaginal tick stages at a consequential rate. As the binomial models showed that the prevalence of infection remains rather constant across wide ranges of tick densities (Figure 3), the risk of infection with B. burgdorferi s.l. is predominantly dependent on the density/activity of ticks (Figure 5).

\section{Coinfection}

One-third of the ticks were infected with one or more pathogens. Additionally, one-third of the ticks infected with Borrelia (234/628) were also infected with at least one other tick-borne pathogen. Recent studies in other European countries have shown that mixed infections of the TBPs do not represent an exception but more likely the rule.

A negative significant association was found between all Borrelia (and $B$. afzelii alone) and $R$. helvetica, as well as between $N$. mikurensis and $R$. helvetica (Table 3). On the other hand, significant positive associations were found between Borrelia (and particularly B. afzelii) and N. mikurensis and between Borrelia and Babesia spp. (Table 3). These findings lead us to the hypothesis that B. afzelii, N. mikurensis, and Babesia share the same reservoir hosts, while $R$. helvetica is maintained in other enzootic cycles.

\section{Seasonal dynamics}

Further evidence for our hypothesis came from the seasonality modelling of the infection prevalence. This indicated a variation in the same phase for $B$. afzelii, N. mikurensis and Babesia spp. on the one side and for non-afzelii $B$. burgdorferi and $R$. helvetica on another (Figure 6). That means that the infection 
peak in questing ticks coincides for some pathogen groups, further suggesting that they were acquired from the same vertebrate hosts. Scientific literature confirms this. Rodents are known to be competent transmission hosts for $B$. afzelii (Gern and Humair 2002a, Hanincova et al. 2003a) and B. microti (Gray et al. 2002), and they have been designated as potential reservoirs for $N$. mikurensis (Andersson and Raberg 2011,Ginsberg 2008). On the other hand, non-afzelii Borrelia, like B. garinii and B. valaisiana have been shown to be associated with birds (Gern and Humair 2002a, Hanincova et al. 2003b), while a study of de la Fuente and co-workers (De La Fuente et al. 2005) found that $A$. phagocytophilum infections occurred in deer, cattle and various bird species, meaning that birds might serve as reservoirs for both these bacteria.

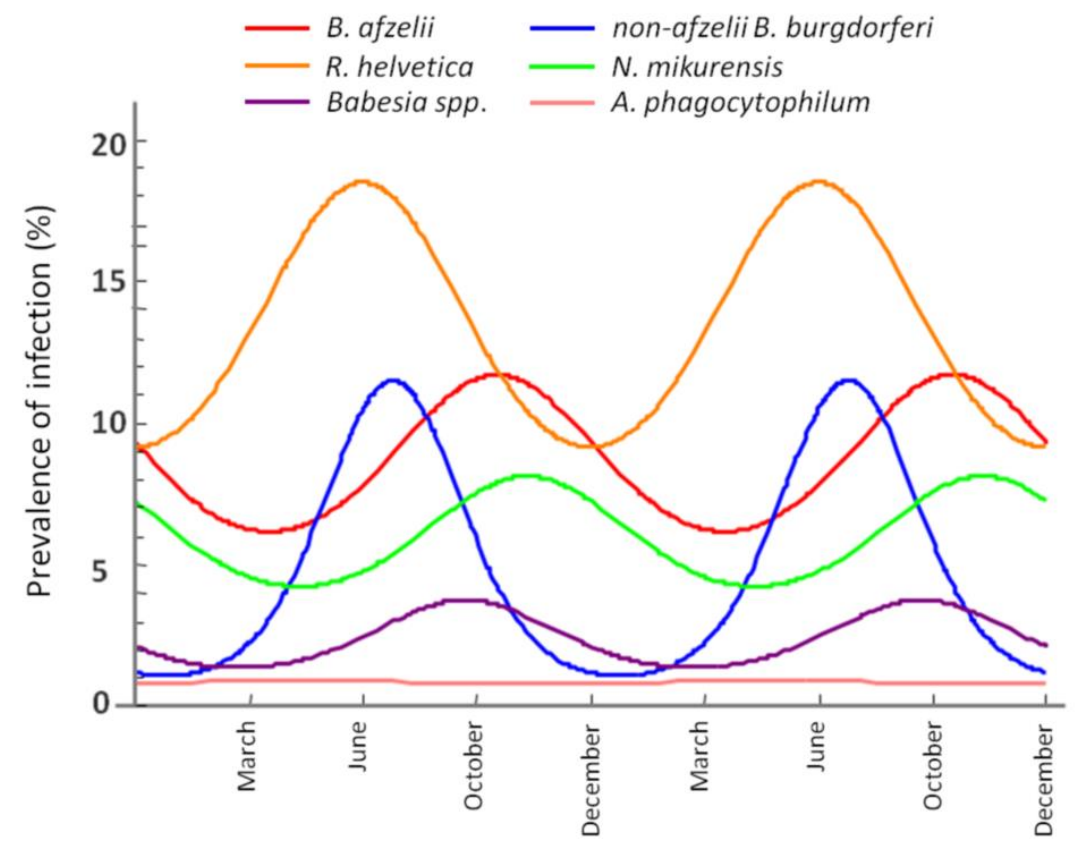

Figure 6. Seasonal variation of the infection rate in ticks. The maximum infection rates of non-afzelii $B$. burgdorferi, and $R$. helvetica are in June, while the maximums of $B$. afzelii, N. mikurensis, and Babesia spp. overlap in October.

R. helvetica was previously found at high rates in both rodents (29\%) and roe deer (19\%)(Sprong et al. 2009). The fact that $R$. helvetica was negatively associated with $B$. afzelii, although they might share the same hosts, is possibly due to that the ticks act as both vectors and reservoirs of the rickettsiae (Sprong et al. 2009), and therefore, they alone can be responsible for the maintenance of the bacteria, without the intervention of a rodent host in the cycle. Hence, our 
findings are not coincidental, and indicate that certain coinfections are more likely to occur than the others, given particular combinations of vertebrate hosts.

Although previous meta-analyses indicate that coinfection and coexposure for some of the TBPs appear to occur somewhat unpredictably across different areas and different hosts (Nieto and Foley 2009), it is anticipated that future wildlife studies will help define geographical risks of coinfection and provide insight into the dynamics of infection within reservoir hosts.

\section{Conclusion}

We have shown that ticks and the five genera of TBPs have a ubiquitous distribution in The Netherlands, with the few absence point presumably determined by the small sample sizes. The pathogens were found in various habitats, with different phytocenoses, from the open areas of dune and heather to the deciduous or coniferous forests.

This study brings valuable information on the prevalence, geographic distribution and temporal variation of $B$. burgdorferi s.l., $R$. helvetica, $N$. mikurensis, A. phagocytophilum and Babesia spp. in questing I. ricinus. Due to their omnipresence, we underline the need to consider all of these pathogens when evaluating the risks of infection and subsequently of disease following a tick bite.

Whereas the incidence of Lyme disease is on the rise, other tick-borne diseases remain heavily unreported, and even knowledge on the human exposure to them is scarce. Our study suggests that there are pathogens positively associated with Borrelia (i.e. N. mikurensis and Babesia spp.) in questing ticks. This strengthens the idea of established enzootic cycles (common reservoir hosts) in which these microorganisms are maintained, and it is consequently possible that they might follow the same upward trend as the Lyme spirochetes. In the case of $N$. mikurensis we have in fact witnessed the beginning of what might be a following upward trend.

Human activity in any natural habitat is accordingly accompanied by an imminent risk of exposure to any of the pathogens. Although the risk, as measured by the density of infected ticks, may vary in time and space, its driving factor appears to be the tick density/activity. It is therefore possible that the risk of exposure to tick-borne pathogens would be minimized by developing effective and sustainable methods for the control of Ixodes ricinus populations. 


\section{Acknowledgements}

The authors are very grateful to the volunteers and co-workers of the Central Veterinary Institute of Wageningen University and Research Centre, particularly Cor Gaasenbeek and Albert de Boer who have dedicated much time and effort to monthly collections of ticks. We are grateful to Christa Drenth for her excellent technical assistance and to Ellen Tijsse-Klasen and Arno Swart for their support and stimulating discussions. This study was financially supported by the Dutch Food and Consumer Product Safety Authority (NVWA), Wageningen University and Research Centre (WUR) and by the Ministry of Health, Welfare and Sport (VWS). 


\section{Chapter 6}

\section{Imbalanced presence of Borrelia burgdorferi s.I. multilocus sequence types}

in clinical manifestations of Lyme borreliosis

This chapter has been published as:

Coipan, E.C., Jahfari, S., Fonville, M., Oei, G.A., Spanjaard, L., Takumi, K., Hovius, J.W., Sprong, H., 2016. Imbalanced presence of Borrelia burgdorferi s.l. multilocus sequence types in clinical manifestations of Lyme borreliosis. Infection, Genetics and Evolution 42, 66-76. 


\begin{abstract}
In this study we used typing based on the eight multilocus sequence typing scheme housekeeping genes (MLST) and 5S-23S rDNA intergenic spacer (IGS) to explore the population structure of Borrelia burgdorferi sensu lato isolates from patients with Lyme borreliosis (LB) and to test the association between the $B$. burgdorferi s.l. sequence types (ST) and the clinical manifestations they cause in humans. Isolates of B. burgdorferi from 183 LB cases across Europe, with distinct clinical manifestations, and 257 Ixodes ricinus lysates from The Netherlands, were analysed for this study alone. For completeness, we incorporated in our analysis also 335 European B. burgdorferi s.I. MLST profiles retrieved from literature. Borrelia afzelii and $B$. bavariensis were associated with human cases of LB while B. garinii, B. lusitaniae and $B$. valaisiana were associated with questing I. ricinus ticks. $B$. afzelii was associated with acrodermatitis chronica atrophicans, while B. garinii and B. bavariensis were associated with neuroborreliosis. The samples in our study belonged to 251 different STs, of which 94 are newly described, adding to the overall picture of the genetic diversity of Borrelia genospecies. The fraction of STs that were isolated from human samples was significantly higher for the genospecies that are known to be maintained in enzootic cycles by mammals (B. afzelii, $B$. bavariensis, and $B$. spielmanii) than for genospecies that are maintained by birds (B. garinii and B. valaisiana) or lizards (B. lusitaniae). We found six multilocus sequence types that were significantly associated to clinical manifestations in humans and five IGS haplotypes that were associated with the human LB cases. While IGS could perform just as well as the housekeeping genes in the MLST scheme for predicting the infectivity of $B$. burgdorferi s.l., the advantage of MLST is that it can also capture the differential invasiveness of the various STs.
\end{abstract}




\section{Introduction}

Lyme borreliosis (LB) is the most prevalent vector-borne disease in Europe (ECDC, 2011), with an incidence that has increased in the past few years (Stanek et al., 2012). The causative agents - Lyme spirochetes - are bacteria belonging to the Borrelia burgdorferi sensu lato complex. They are transmitted by ticks of genus Ixodes (Burgdorfer et al., 1982) and are maintained in enzootic cycles by different vertebrate hosts (Kurtenbach et al., 2006; Mannelli et al., 2012).

The disease presents itself under a wide range of clinical manifestations in humans. These manifestations start, in many cases, with erythema migrans (EM) - a rash at the site of the tick bite, and progress on some occasions towards disseminated manifestations such as cardiovascular, neurologic, or arthritic ones. At least five genospecies of $B$. burgdorferi s.l. have been shown to be pathogenic to humans - B. afzelii, B. garinii, B. burgdorferi sensu stricto, $B$. spielmanii, and $B$. bavariensis (Stanek et al., 2012). Current knowledge is that $B$. afzelii is predominantly involved in cutaneous manifestations, being often found in the localized incipient form of the infection and in acrodermatitis chronica atrophicans (ACA), B. garinii in neurological manifestations, and B. burgdorferi in articular ones (Stanek et al., 2012). Thus, these two last genospecies are, apparently, more invasive, being able to disseminate from the inoculation site to other tissues or/and to survive the early immune response. However, there is not a clear-cut differentiation between members of these genospecies in terms of symptoms. Next to that, there have been reports of disseminated disease (e.g. neuroborreliosis (NB)) without any characteristic erythema migrans symptoms (Reik et al., 1986); that would imply that, while being more invasive, some strains can be overlooked when only testing EM biopsies.

Furthermore, it is not yet known if all the Borrelia genotypes within the pathogenic genospecies present in questing ticks can cause disease in humans or if there is only a subset of them that is pathogenic (infectious). From a public health perspective, it is important to be able to differentiate between the infectious and non-infectious Borrelia spirochaetes or between the invasive and non-invasive ones. Discriminating between these types could be useful for disease risk assessment and management.

Research on B. burgdorferi s.s. in North America has shown that some major sequence types of the outer surface protein $C(o s p C)$ and certain sequence types of 16S-23S rRNA intergenic spacer are more frequently found in disseminated cases of LB (Dykhuizen et al., 2008; Strle et al., 2011; Wormser et al., 2008). More recently, Hanincova et al. (2013) have shown significant 
associations between clusters of sequence types (clonal complexes) of $B$. burgdorferi s.s. and localized or disseminated forms of LB. It seems, thus, that the genetic makeup of the pathogenic spirochetes is determinant for the symptomatology they cause. Consequently, genotyping the bacteria might hold the answer to the question of differential infectivity and invasiveness of $B$. burgdorferi s.l.

The gold standard for genotyping of $B$. burgdorferi nowadays is multilocus sequence typing (MLST), based on eight housekeeping genes on the chromosome, which undergo slow evolution and show nearly neutral variation (Margos et al., 2008; Urwin and Maiden, 2003). Previous studies (Coipan et al., 2013a) have shown that the 5S-23S rDNA intergenic spacer (IGS) can also discriminate among the genospecies of $B$. burgdorferi s.l. and that it can also detect genetic differentiation among the bacteria of various geographic provenience.

In this study we used typing based on MLST and IGS to explore the population structure of $B$. burgdorferi s.l. isolates from patients with LB and to address the issue of association between the $B$. burgdorferi s.l. sequence types (ST) and the clinical manifestations they cause in humans. Firstly, we examined whether all the bacterial STs found in questing ticks can cause disease in humans or it is only a small subset that is responsible for the reported cases of LB. Assuming that the pathogenicity of the bacteria is a characteristic determined primarily by their genetic composition, we used MLST and IGS as genetic proxies for infectivity/invasiveness of a strain. Secondly, we wanted to test whether there are $B$. burgdorferi s.l. STs that are more frequently encountered in human cases than we would expect based on their frequency in questing ticks. If that were to be true, it would imply that some of the bacterial STs are more infectious than others. The last hypothesis we tested is that of differential dissemination/persistence ability of $B$. burgdorferi s.l., for which we calculated the probabilities of various STs of causing disseminated/persistent clinical manifestations in humans.

\section{Material \& Methods}

\section{Borrelia isolates}

Isolates of B. burgdorferi from 183 Lyme borreliosis cases across Europe, with distinct clinical manifestations, and 257 Ixodes ricinus lysates from The Netherlands, were analysed for the present study. The European isolates were selected primarily to give as wide as possible diversity of Borrelia in human samples, based on their country of origin. 


\section{DNA extraction and screening of questing ticks}

DNA extraction from the individual questing ticks was done by alkaline lysis in ammonium hydroxide, as described previously (Schouls et al., 1999), while the DNA extraction from the bacterial cultures was performed using DNeasy ${ }^{\circledR}$ Blood \& Tissue Kit (QIAGEN N.V., Venlo, The Netherlands). Screening of the questing ticks for $B$. burgdorferi s.l. was done by $\mathrm{qPCR}$ with outer surface protein $A(o s p A)$ and flagellin $B(f l a B)$ targeted primers, as described (Heylen et al., 2013). The majority of the ticks included in this study was represented by nymphs, as they constitute most of the acarological risk (Coipan et al., 2013b) for acquiring LB.

\section{Multilocus sequence typing (MLST)}

All 440 isolates of $B$. burgdorferi s.l. were sequenced and typed using the MLST procedure described by (Margos et al., 2008), except that the elongation times were $60 \mathrm{~s}$. Briefly, eight loci on the bacterial chromosome (clpA, clpX, nifS, pepX, pyrG, rplB, recG, and uvrA) were amplified and subsequently sequenced in forward and reverse directions. Trimming and manual cleaning of sequences was performed in Bionumerics 7.1. (Applied Math, Belgium). In frame alignment of sequences was made with TranslatorX (Abascal et al., 2010), using the program MUSCLE (Edgar, 2004) with bacterial and plant plastid genetic code.

\section{S-23S typing}

For 220 of these samples we also performed PCR targeting the variable 5S-23S rDNA intergenic spacer region (IGS). The PCR was performed according to the protocol described in Coipan et al. (Coipan et al., 2013a). Alignment of the sequences was made using MAFFT (Katoh and Toh, 2008) and the sequences were trimmed to nucleotides between position 438838 and 439196 (359 nucleotides) of whole genome sequence of $B$. afzelii strain PKo (Genbank entry CP002933). An IGS sequence type was defined here as a group of Borrelias within a genospecies in which all members share an identical sequence. We assigned a unique number to each of the IGS sequence types.

\section{Multilocus sequence analysis}

Each unique allele type of the housekeeping genes received a number. Alleles that were identical to the ones already existing in MLST.net received the same number, while the new ones received numbers over 500 . Similarly, the STs resulting from concatenating the alleles at all eight loci, were assigned unique numbers that either matched the known ones in MLST.net or had values higher than 1000 if they were previously unknown. 


\section{Chapter 6}

To gain more information regarding the distribution of the STs in questing ticks we incorporated in our analysis also 335 European B. burgdorferi s.l. MLST profiles that we retrieved from publications (Hoen et al., 2009; Margos et al., 2008; Margos et al., 2009; Vollmer et al., 2011), the full dataset analysed consisting, thus, of 775 isolates.

\section{Phylogenetic analysis and clustering}

The cluster analysis was performed on the IGS sequences and on each of the eight MLST loci, as well as on the concatenated sequences of the latter ones. Best-scoring maximum likelihood trees were generated using the PhyML online platform (Guindon et al., 2010), with a general time reversible (GTR) model of DNA evolution and Subtree-pruning-regrafting (SPR) and Nearest Neighbor Interchanges (NNI) for tree improvement, with 100 bootstraps. We identified clusters of Borrelia isolates in the phylogenetic tree. For this, we retained, using Biopython 1.65, only the clusters with a strong bootstrap support (i.e. greater than 90 percent) in the best-scoring phylogenetic tree.

\section{Rarefaction analysis}

To test our hypothesis of the differential pathogenicity (whether all the Borrelia spirochetes found in ticks are infectious to humans or the ones that are found in clinical manifestations are only a subset) we counted distinct STs of $B$. burgdorferi s.l. identified in clinical samples. Similarly, we counted distinct STs identified in tick samples. We ran a rarefaction analysis, implemented in Estimates 9 (Colwell, 2013) for individual-based abundance data, with 100 runs, randomization of individuals without replacement, and extrapolation to 2000 individuals. We performed the analysis for both, the human and the tick datasets, to determine the species richness of the two samples.

\section{Statistical analysis}

Descriptive statistics (haplotype diversity, nucleotide diversity, synonymous and nonsynonymous polymorphisms) were determined using DnaSP 5.10.01.

Extent to which human source was overrepresented in a particular ST can be quantitatively evaluated by calculating the probability of observing more clinical samples of the particular ST than actually observed in the samples. For this, we evaluated beta binomial cumulative distribution using a function pbetabinom.ab ( $q$, size, shape1, shape2, log.p = FALSE) implemented in the R package VGAM. Function arguments are: the number of clinical samples $(q)$ and the total number of samples (size) in a particular ST, the number of clinical 
samples (shape1) and the number of tick samples (shape2) in the most abundant ST. Hence, excess of human source in the particular ST was evaluated against the most abundant ST (= reference). Using the same methodology, we also evaluated extent to which any of the three clinical manifestations - EM, ACA, NB was overrepresented in a particular ST.

To check whether also the reference STs, genetic clusters, or genospecies were associated with either human cases or disseminated/persistent forms of the disease we ran a Fisher's exact test. We tested at a turn each ST against the totality of the other ones in human versus ticks; we repeated the test for each of the disseminated/persistent clinical manifestations against erythema migrans. The null hypothesis was that the frequency of the STs should be equal in any two different sources or symptoms. The correction for multiple testing was performed according to the method described by Benjamini and Hochberg (1995). The same procedure we used for the genetic clusters and genospecies.

Since the majority of the human samples came from bacterial cultures, while the majority of the tick samples were lysates of the ticks, in order to be able to compare the frequencies of the STs in these samples we assume that all genotypes are equally likely to be cultured and sequenced. Should this assumption not hold, the results of the comparison between the ticks and human isolates might be biased and should, therefore, be interpreted with caution.

The main assumption when combining DNA sequences from all across Europe in one analysis is that there is no significant genetic differentiation based on the geographic location. This is especially important for the human samples. Since the results of the beta-binomial test is based on the existence of rare variants in ticks that are overrepresented in human samples, it is important to exclude the possibility that any significant results are due to the absence of sampling from the tick population (see Supplementary table 1 for country of origin of human and tick samples) and the existence of genetic differentiation in the human samples between distinct countries. To exclude that possibility, we tested for genetic differentiation using the "Comparisons of pairs of population samples" test implemented in Arlequin 3.5.2.1 (Excoffier and Lischer, 2010). The results were interpreted based on the matrix of significant Fst $p \mathrm{~s}$, with a significance level of 0.05 .

Comparison between MLST and IGS predictive values for pathogenicity of B. burgdorferi s.l.

We applied principal component analysis implemented in smartpca (Patterson et al., 2006) to Borrelia isolates sampled in 161 patients and 24 


\section{Chapter 6}

vegetation ticks; these were the samples from which we had sequences of the eight housekeeping genes as well as the IGS. Our purposes were to: (1) distinguish the group of Borrelia isolates sampled in patients from those sampled in vegetation ticks, (2) identify SNPs that are informative in delineating the subpopulations, and (3) identify the genes that contain the informative SNPs. The differentiation between the Borrelia populations in the two different sources was tested with a $\chi^{2}$ test. By running smartpca, we calculated ANOVA statistics for population differences along each eigenvectors and associated $p s$. We calculated also the weights of SNPs along each eigenvectors. The sequences used in the analysis were the concatenated sequences of the eight MLST loci and IGS; the resulting sequences were 5157 nucleotides (nt), containing 765 SNPs. Following the alignment, we coded each nucleotide by a biallelic state: "1" when the base was identical to the reference base, and " 0 " for nucleotides different from the one in the reference sequence. As reference, we chose the most common ST in the dataset. The settings for the smartpca included turning the option killr2 on and setting the option r2thresh equal to 0.5 in order to eliminate SNPs in linkage disequilibrium with nearby SNPs having correlation coefficient greater than 0.5 .

Next, we performed similar analyses using the concatenated MLST genes (4788 nt) of the Borrelia samples from 193 patients; these were all the human samples from which we had a fill MLST profile. The total number of SNPs contained by these was 1120 . In this case we labelled each sample by the clinical manifestation of the patient from whom the bacteria were cultured. Our purposes were to: (1) distinguish the group of Borrelia isolates sampled in neuroborreliosis and acrodermatitis chronica atrophicans patients from those sampled in erythema migrans patients, and (2) identify informative SNPs.

\section{Results}

\section{Genospecies diversity}

The full data set that we used in our analysis consisted of 775 entries 440 from this study and 335 from the literature. Approximately half of the $\mathbf{7 7 5}$ samples were $B$. afzelii $(n=390)$. Borrelia garinii represented approximately a quarter of the samples $(\mathrm{n}=191)$. Borrelia valaisiana accounted for approximately $13 \%$ of the samples $(n=99)$, while the rest of the genospecies were each represented by less than $5 \%$ of the entries (Table 1). The 335 sequences retrieved from the literature were in overwhelming majority from ticks $(n=310)$ and only $7.5 \%$ were from human Lyme borreliosis cases $(n=25)$. Of the total 440 samples tested for this study alone, sequencing at all eight loci was successful for 293 , with an even distribution among the two sources: $58 \%$ 
from humans $(n=170)$ and $42 \%$ from Ixodes ricinus ticks $(n=123)$. Nevertheless, all the samples could be attributed to a certain Borrelia genospecies. The total number of isolates in our analysis of which we had a complete MLST profile was 628.

Table 1. Source of samples and MLST profiles. The number in each cell is the total number of samples used in the analyses while the number in the brackets refers to the total number of the samples sequenced for this study alone. $\mathrm{AF}=\mathrm{B}$. afzelii, $\mathrm{BA}=\mathrm{B}$. bavariensis, $\mathrm{BI}=B$. bissettii, $\mathrm{BU}=B$. burgdorferi sensu stricto, $\mathrm{GA}=B$. garinii, $\mathrm{LU}=B$. lusitaniae, $\mathrm{SP}=B$. spielmanii, $\mathrm{VA}=B$. valaisiana, $\mathrm{EM}=$ erythema migrans, $\mathrm{ACA}=$ acrodermatitis chronica atrophicans, $\mathrm{NB}=$ neuroborreliosis and LA = Lyme arthritis.

\begin{tabular}{lrrrrrr}
\hline Genospecies & tick & EM & ACA & NB & LA & Total \\
\hline AF & $229(157)$ & $122(115)$ & $37(36)$ & 0 & $2(2)$ & $\mathbf{3 9 0 ( 3 1 0 )}$ \\
BA & $3(1)$ & $9(6)$ & 0 & $13(7)$ & 1 & $\mathbf{2 6 ( 1 4 )}$ \\
BI & 1 & 0 & 0 & 0 & 0 & $\mathbf{1}$ \\
BU & $28(4)$ & $9(9)$ & $1(1)$ & 0 & 0 & $\mathbf{3 8 ( 1 4 )}$ \\
GA & $158(44)$ & $24(24)$ & 0 & $9(4)$ & 0 & $\mathbf{1 9 1 ( 7 2 )}$ \\
LU & $16(2)$ & 1 & 0 & 0 & 0 & $\mathbf{1 7}(2)$ \\
SP & $10(7)$ & $3(2)$ & 0 & 0 & 0 & $\mathbf{1 3 ( 9 )}$ \\
VA & $99(19)$ & 0 & 0 & 0 & 0 & $\mathbf{9 9 ( 1 9 )}$ \\
\hline Total & $\mathbf{5 4 4 ( 2 3 4 )}$ & $\mathbf{1 6 8 ( 1 5 6 )}$ & $\mathbf{3 8 ( 3 7 )}$ & $\mathbf{2 2 ( 1 1 )}$ & $\mathbf{3 ( 2 )}$ & $\mathbf{7 7 5 ( 4 4 0 )}$ \\
\hline
\end{tabular}

Next to The Netherlands ( $n=363$ ), the European countries that were best represented in this data set were Great Britain $(n=120)$, Latvia $(n=81)$, France ( $n=62)$, and Germany ( $n=44$; Supplementary Table 1$)$.

Regarding the sample source, more than two thirds of the entries were from ticks ( $n=544$; Table 1 ). The human samples were mostly retrieved from the incipient form of LB - erythema migrans (EM; $n=168$ ). Among the disseminated forms of LB, acrodermatitis chronica atrophicans (ACA) was the most frequent, with $4.9 \%$ of all cases, while neuroborreliosis (NB) represented approximately half of that $(2.84 \%)$. Lyme arthritis (LA) was the least frequent form of disseminated disease $(0.39 \%)$ in our dataset. Eleven isolates were retrieved from both EM and one of the late manifestations LB (one ACA, one LA, and nine NB); these were included twice in the dataset, once for each of the symptoms.

\section{Intra-genospecies diversity}

Out of the 628 samples sequenced at all eight housekeeping loci, 16 showed double peaks in at least one of the genes. These were eight $B$. afzelii, six $B$. garinii, and two $B$. bavariensis samples and they were further excluded from 


\section{Chapter 6}

the assignation of STs, being used only for the cluster analyses. The presence of double peaks in the DNA sequences is indicative of multiple infection of the ticks with different Borrelia STs. The exclusion of this data might have negatively affected the frequency of some STs in questing ticks. However, we expect that the low number (16) would not significantly impact the results of our analysis.

Overall, the samples in our study belonged to 251 different STs (Table 2a), of which 94 are newly described. Fasta files of the newly described STs are available upon request. The majority of the newly described STs belong to $B$. afzelii (74) followed by B. garinii (13; Table 2a).

However, the within genospecies genetic differentiation varied broadly, with very short branches for $B$. afzelii and long ones for $B$. garinii (Figure 1 ). This is also supported by summary statistics for each genospecies (Table 2a): The haplotype diversity was highest for $B$. afzelii $(0.9845)$ and slightly smaller for $B$. burgdorferi and $B$. garinii (Table 2a) while the nucleotide diversity was low for $B$. afzelii (0.00248), but high for B. burgdorferi $(0.00659)$ and B. garinii $(0.00884)$. All these metrics indicate the existence of a gradient of STs for $B$. afzelii and higher genetic differentiation for $B$. garinii and B. burgdorferi. Borrelia bavariensis was the genospecies with the lowest haplotype diversity $(0.181)$ and the lowest nucleotide diversity (0.00004; Table 2a).

Cluster analysis revealed robust clustering of the entries belonging to the same genospecies (Figure 1), with bootstrap values of 100 for most of the genospecies. The pathogenic bacteria were unevenly distributed among the branches of the tree, with an almost homogeneous distribution within B. afzelii and an imbalanced one within B. garinii (Figure 1). Similarly, the STs isolated from late and disseminated manifestations of LB were unevenly distributed. While STs of bacteria causing ACA were present almost exclusively in $B$. afzelii and in a very low number in $B$. burgdorferi, the STs isolated from NB were very well represented within $B$. bavariensis and to a smaller extent within $B$. garinii (Figure 1).

Figure 1. Cluster analysis of B. burgdorferi s.l. isolates based on housekeeping genes' MLST. The clinical manifestations are highlighted in dark blue in each of the corresponding columns in the heatmap attached to the tree: EM, ACA, NB, and LA. The grey background stands for the tick samples and all other human samples that are not in the category designated by the column name. $\rightarrow$ 


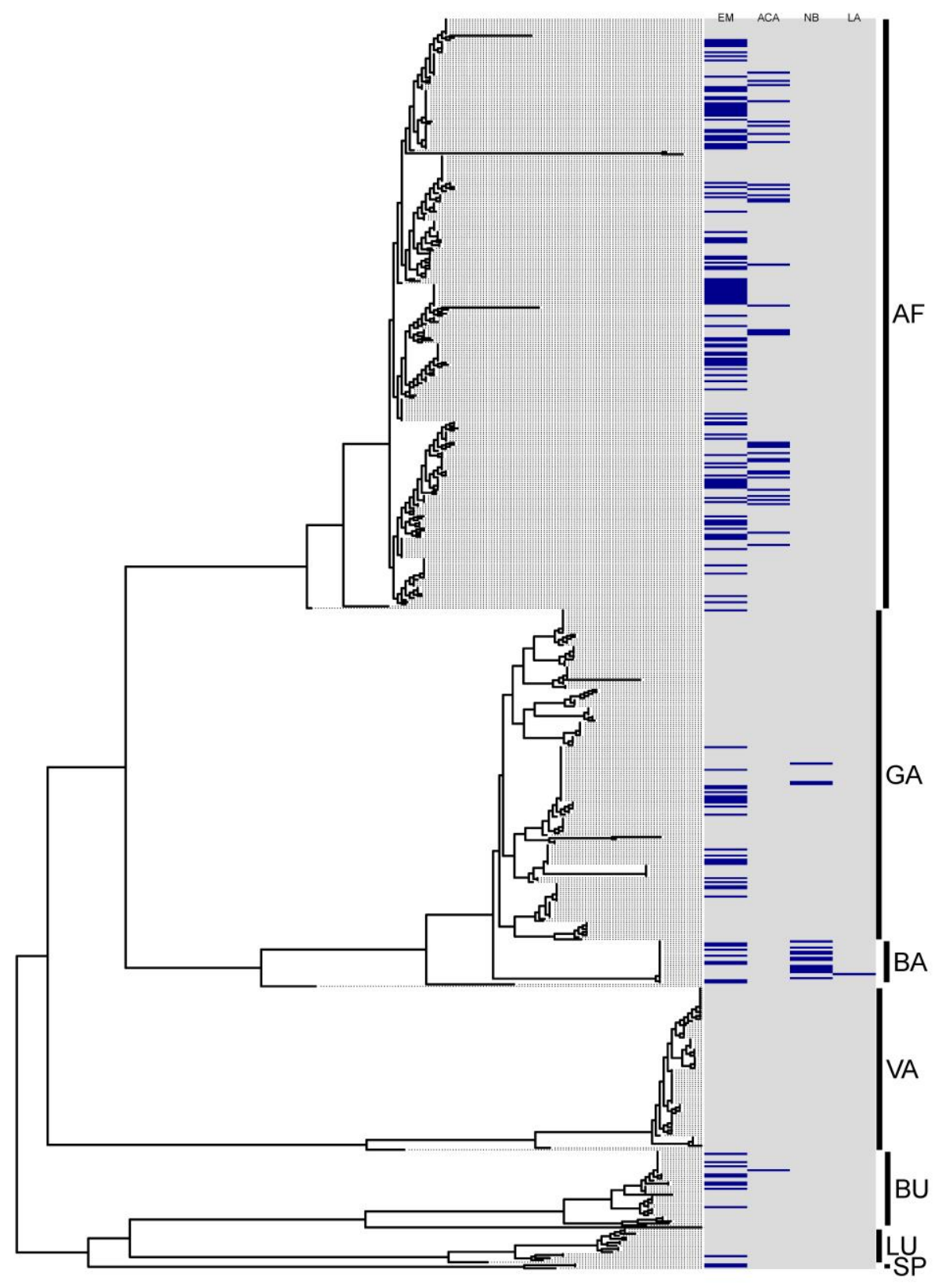


Table 2. Summary statistics for intragenospecies divergence of $B$. burgdorferi s.l. based on the eight MLST loci. S.L. $=B$. burgdorferi s.I., AF $=B$. afzelii, $\mathrm{BA}=B$. bavariensis, $\mathrm{BU}=B$. burgdorferi, $\mathrm{GA}=B$. garinii, $\mathrm{LU}=B$. lusitaniae, $\mathrm{VA}=\mathrm{B}$. valaisiana

a) Values for full dataset and for each genospecies (B. spielmanii and $B$. bissettii are omitted from the table)

\begin{tabular}{|c|c|c|c|c|c|c|c|}
\hline Population used & S.L. & $\mathrm{AF}$ & BA & & $\overline{G A}$ & LU & VA \\
\hline Number of sequences & 612 & 289 & 21 & & 164 & 17 & 00 \\
\hline Number of sequence types* & 251 & 129 & 2 & & 17 & 13 & 28 \\
\hline Number of new sequence types & 94 & 74 & 0 & & 3 & 2 & 2 \\
\hline $\begin{array}{l}\text { Total number of sites (-sites with } \\
\text { gaps) }\end{array}$ & 4782 & 4785 & 4785 & 4785 & 4782 & 4785 & 4785 \\
\hline Number of polymorphic sites & 1120 & 332 & 1 & 150 & 370 & 134 & 196 \\
\hline Total number of mutations & 1284 & 336 & 1 & 150 & 381 & 137 & 198 \\
\hline Haplotype (gene) diversity, Hd & 0.9908 & 0.9845 & 0.181 & 0.896 & 0.955 & 0.971 & 0.93 \\
\hline Standard Deviation of $\mathrm{Hd}$ & 0.0009 & 0.002 & 0.104 & 0.039 & 0.009 & 0.028 & 0.015 \\
\hline Nucleotide diversity, $\mathrm{Pi}$ & $\begin{array}{r}0.0460 \\
7\end{array}$ & $\begin{array}{r}0.0024 \\
8\end{array}$ & $\begin{array}{r}0.0000 \\
4\end{array}$ & 0.0065 & $\begin{array}{r}0.0088 \\
4\end{array}$ & $\begin{array}{r}0.0079 \\
4\end{array}$ & $\begin{array}{r}0.0027 \\
7\end{array}$ \\
\hline Standard deviation of $\mathrm{Pi}$ & 0.0009 & $\begin{array}{r}0.0002 \\
6\end{array}$ & $\begin{array}{r}<0.000 \\
1\end{array}$ & 0.001 & $\begin{array}{r}0.0004 \\
2\end{array}$ & $\begin{array}{r}0.0014 \\
7\end{array}$ & $\begin{array}{r}0.0007 \\
9\end{array}$ \\
\hline \multicolumn{8}{|c|}{$\begin{array}{l}\text { b) Values for the different sources (human and tick) of the main three } \\
\text { genospecies }\end{array}$} \\
\hline \multirow{2}{*}{ Population used } & \multicolumn{2}{|c|}{ B. afzelii } & \multicolumn{3}{|c|}{ B. burgdorferi s.s. } & \multicolumn{2}{|c|}{ B. garinii } \\
\hline & human & tick & & uman & tick & human & tick \\
\hline Number of sequences & 135 & 154 & & 10 & 27 & 25 & 139 \\
\hline Number of sequence types* & 68 & 78 & & 5 & 15 & 8 & 57 \\
\hline Total number of sites (-sites with gaps) & 4785 & 4785 & & 4785 & 4785 & 4782 & 4782 \\
\hline Number of polymorphic sites, $\mathrm{S}$ & 68 & 313 & & 38 & 142 & 75 & 369 \\
\hline Total number of mutations, Eta & 68 & 316 & & 38 & 142 & 75 & 380 \\
\hline Haplotype (gene) diversity, $\mathrm{Hd}$ & 0.976 & 0.978 & & 0.822 & 0.917 & 0.77 & 0.968 \\
\hline Standard deviation of $\mathrm{Hd}$ & 0.005 & 0.004 & & 0.097 & 0.038 & 0.07 & 0.006 \\
\hline Nucleotide diversity, $\mathrm{Pi}$ & 0.00196 & 0.00292 & & 0211 & 0.00772 & 0.00417 & 0.00942 \\
\hline Standard deviation of $\mathrm{Pi}$ & $<0.0001$ & 10.00048 & & 00087 & 0.0013 & 0.00064 & 0.00047 \\
\hline
\end{tabular}

*sequence type $=$ haplotype

Not all the STs that were present in tick samples (194) were also retrieved from human cases (85). Likewise, not all the STs that were found in EM (72) were also found in late or disseminated LB (28; Supplementary table 1). The haplotype and nucleotide diversity of the main three genospecies (B. afzelii, B. garinii, and $B$. burgdorferi) were, consequently, constantly lower in the human than in the tick samples (Table 2 b) e.g. haplotype diversity of $B$. garinii in humans was only 
0.77 , compared to 0.968 in ticks, while nucleotide diversity was only 0.00417 in humans and 0.00942 in ticks.

\section{Rarefaction analysis}

To compare ST diversities of spirochetes from different sources we ran EstimateS for all B. burgdorferi s.l. genospecies in our data set. The result indicated a much higher diversity of the STs from ticks compared to those from human cases. Confidence intervals are non-overlapping (Figure 2). Even after rarefying the tick sample to 192 individuals to match the size of the human sample, the ST richness was greater in the tick sample. Thus, on average we expect to identify 85 distinct STs in 192 human samples while 115 distinct STs are expected in the same number of tick samples. Similar results were obtained for $B$. garinii and B. burgdorferi (although with broad confidence intervals for the second one; Supplementary Figures $2 b, c)$. For $B$. afzelii, the number of distinct STs of the bacteria isolated from human cases was only slightly smaller than that from ticks; the overlapping confidence intervals of the two distributions indicate that there is no significant difference between the diversity of two sources (Supplementary Figure 2a).

\section{Associations of STs with source of samples}

Borrelia afzelii and B. bavariensis were found to be positively associated with human cases of LB while $B$. garinii, $B$. lusitaniae and $B$. valaisiana were positively associated with questing $I$. ricinus ticks (Fisher's test: Table 3a). The fraction of STs isolated from human samples was significantly higher for the genospecies that are known to be maintained in enzootic cycles by mammals ( $B$. afzelii, $B$. bavariensis, and $B$. spielmanii) than for genospecies that are maintained by birds (B. garinii, and B. valaisiana; Fisher's exact test $p=6.4 \times 10^{-5}$ ) or lizards ( $B$. lusitaniae; Fisher's exact test $p=7.9 \times 10^{-3}$ ). The difference was still significant even when we added the STs of $B$. burgdorferi s.s. and B. bissettii to either mammal or bird transmitted Borrelia or both (Fisher's exact test $p<$ $\left.1.8 \times 10^{-5}\right)$.

There were six STs that were significantly associated to clinical manifestations in humans $(71,84,171,476,1071$, and 1080; Table 3b). Despite the fact that these were not the most abundant STs in questing ticks, they were still frequently found in human cases. There were also differences between genospecies in terms of association with late or disseminated clinical manifestations of LB. Thus, some STs of $B$. afzelii were positively associated with acrodermatitis chronica atrophicans (beta-binomial test: Table $3 \mathrm{c}$ ), while $B$. garinii (Fisher's exact test on Table $1, p=4.8 \times 10^{-3}$ ) and B. bavariensis (Table $3 \mathrm{~d}$ ) were positively associated with neuroborreliosis. 


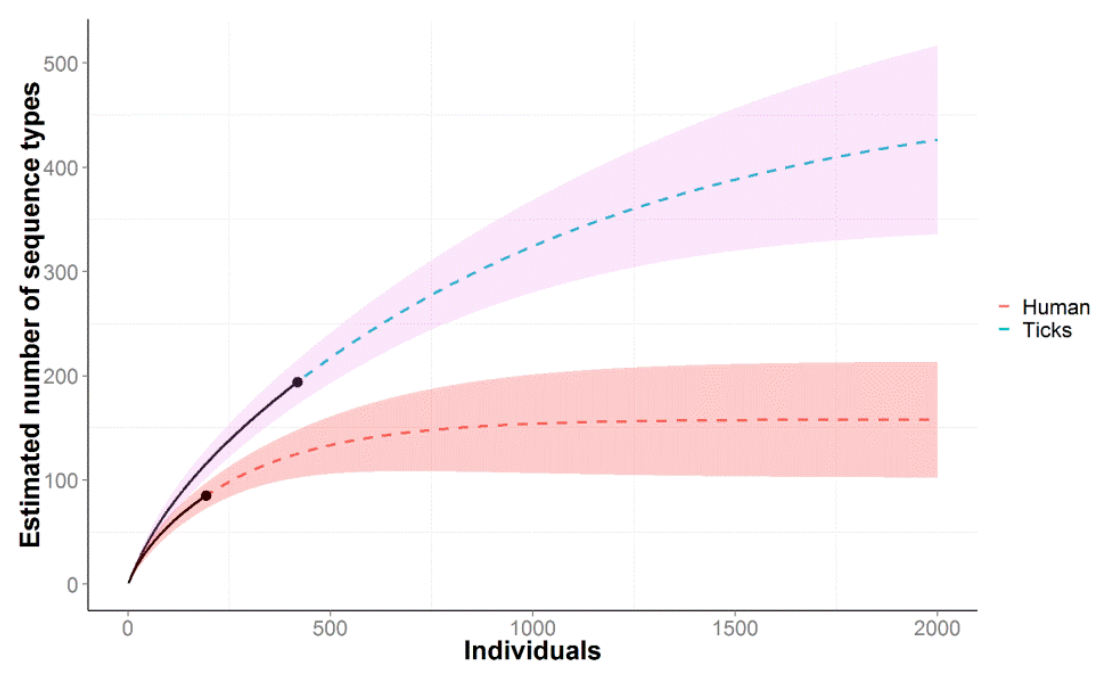

Figure 2. Individual-based rarefaction (solid curves) and extrapolation (dashed curves) from tick and human samples (filled black circles), indicating the $\mathrm{ML}$ sequence type richness in the two samples. On the $\mathrm{x}$-axis the number of sampled individuals is counted (419 individuals for ticks and 192 individuals for humans), while on the $y$-axis the corresponding number of STs is depicted.

Following the inspection of the phylogenetic tree we noticed that the Borrelia isolated from humans were not evenly distributed among all the branches. Consequently, we wanted to see if there is a higher probability of a branch or a cluster to cause LB. To further test our hypothesis of differential pathogenicity of $B$. burgdorferi s.l., we compared the frequencies of the different genospecies of Borrelia in disseminated/persistent forms of the disease - ACA and NB - with the localized form - EM.

We showed that there are differences between STs of the same genospecies in terms of their capacity to cause late or disseminated forms of LB. The comparison between ACA and EM based on the beta-binomial test indicated that ST 335, 1054, and 1073 are significantly more frequent in late LB than ST1071 - the most abundant ST in the EM cases, that can also cause ACA (Table $3 c)$. When tested for association with EM based on their frequency in questing ticks, these STs were not significantly associated with the incipient form of LB.

The strongest indication that a ST is particularly invasive was in the case of ST 84 of B. bavariensis, which was positively associated with NB (Table 3d), while ST 86 (B. garinii) was also frequent in NB cases. ST 84 was also significantly more frequent in $\mathrm{EM}$ cases than in questing ticks (beta-binomial test $p=0.015$ ) 
Table 3. Associations of genospecies and sequence types with source of isolation or clinical manifestations. The genospecies or sequence types that have a probability lower than 0.05 are considered to be significantly associated to human cases of Lyme borreliosis or to the disseminated form of the disease (ACA or NB). The columns "human", "tick", "ACA", "EM", and "NB" represent the number of isolates from each of those sources/clinical manifestations corresponding to each of the genospecies and/or sequence types. The values in columns Fisher's test and beta-binomial represent the p-values of those two statistical tests.

a) genospecies associations with source of isolation

\begin{tabular}{lrrrr}
\hline Genospecies & human & tick & Fisher's test & beta-binomial \\
\hline AF & 161 & 229 & $1.76 \times 10^{-12}$ & reference \\
BA & 23 & 3 & $3.75 \times 10^{-10}$ & $1.7 \times 10^{-5}$ \\
BI & 0 & 1 & 1 & 1 \\
BU & 10 & 28 & 0.72 & 0.98 \\
GA & 33 & 158 & $7.46 \times 10^{-6}$ & 1 \\
LU & 1 & 16 & 0.03 & 0.99 \\
SP & 3 & 10 & 0.76 & 0.95 \\
VA & 0 & 99 & $3.92 \times 10^{-17}$ & 1 \\
\hline
\end{tabular}

b) sequence type association with source of isolation

\begin{tabular}{lrrrr}
\hline Sequence type & Genospecies & human & tick & beta-binomial \\
\hline $\mathbf{8 6}$ & B. garinii & 11 & 16 & reference \\
$\mathbf{7 1}$ & B. afzelii & 4 & 0 & $3.6 \times 10^{-2}$ \\
$\mathbf{8 4}$ & B. bavariensis & 18 & 1 & $9.4 \times 10^{-4}$ \\
$\mathbf{1 7 1}$ & B. afzelii & 8 & 1 & $1.1 \times 10^{-2}$ \\
$\mathbf{4 7 6}$ & B. afzelii & 11 & 0 & $4.1 \times 10^{-4}$ \\
$\mathbf{1 0 7 1}$ & B. afzelii & 12 & 3 & $1.1 \times 10^{-2}$ \\
$\mathbf{1 0 8 0}$ & B. afzelii & 5 & 0 & $1.7 \times 10^{-2}$ \\
\hline
\end{tabular}

c) sequence type association with ACA

\begin{tabular}{lrrrr}
\hline Sequence type & Genospecies & ACA & EM & beta-binomial \\
\hline $\mathbf{1 0 7 1}$ & B. afzelii & 1 & 11 & reference \\
$\mathbf{3 3 5}$ & B. afzelii & 2 & 1 & $3.2 \times 10^{-2}$ \\
1054 & B. afzelii & 2 & 0 & $1.3 \times 10^{-2}$ \\
1073 & B. afzelii & 3 & 0 & $2.7 \times 10^{-2}$ \\
\hline
\end{tabular}

d) sequence type association with NB

\begin{tabular}{lrrrr}
\hline Sequence type & Genospecies & NB & EM & beta-binomial \\
\hline $\mathbf{8 6}$ & B. garinii & 3 & 8 & reference \\
$\mathbf{8 4}$ & B. bavariensis & 11 & 6 & $3 \times 10^{-2}$ \\
\hline
\end{tabular}




\section{Chapter 6}

The clusters obtained by collapsing the branches of the phylogenetic tree (Supplementary Figure 1) were also tested for association with human cases. In the case of $B$. garinii none of the clusters showed significant association with any of the human or tick source. Cluster " 6 " of $B$. afzelii was significantly associated with human cases (Fisher's exact test $p=0.0416$ ). Cluster " 2 " of $B$. burgdorferi showed significant positive association (Fisher's exact test $p=0.0229$ ) with human LB. There was no predilection of any of these clusters for a certain disseminated clinical manifestation of LB.

In our study, we identified, thus, two genospecies ( $B$. afzelii and $B$. bavariensis), two phylogenetic clusters (" 6 " of $B$. afzelii and " 2 " of $B$. burgdorferi), and six MLST types (84 of B. bavariensis and 71, 171, 476, 1071, 1080 of B. afzelii) positively associated with human LB. Additionally, we identified four MLST types positively associated with disseminated LB: ST 335, 1054 and 1073 of B. afzelii and ST 84 of B. bavariensis.

The pair comparison of population samples indicated that there was no significant genetic differentiation between the various European countries for any of the Borrelia genospecies, except for $B$. afzelii. The population of $B$. afzelii from Italy was significantly different from those from Denmark and Sweden, but also that from The Netherlands was significantly different from those from France, Germany and Austria. Since in some of these countries (Austria, Italy, and Sweden; Supplementary table 1) there were no MLST profiles retrieved from the questing ticks, one might argue that this is the reason underlying the association of some STs to human cases. We excluded this possibility because all the STs that were associated with human cases came from countries where also tick samples were available from.

For the 5S-23S ribosomal DNA spacer, 21 of the 220 sequences contained ambiguous nucleotides and were removed from further analysis. The remaining 199 samples belonged to 36 different haplotypes, of which 25 were of $B$. afzelii, six of $B$. garinii, two of each $B$. bavariensis and B. burgdorferi s.s., and one of $B$. spielmanii. Their distribution over $B$. burgdorferi s.l. genospecies, the source of isolation, and their corresponding MLST ST is shown in Table 4.

The genetic variability of IGS was, on average, higher than that of the individual MLST housekeeping genes for the same samples but lower than that of their concatenated sequences. IGS haplotypes corresponded to 86 multi-locus STs. By applying a beta-binomial test on a larger dataset of IGS (not shown here), comprising also sequences from questing ticks, there were five haplotypes -28 , $71,95,121$ and 141 - that were positively associated to the human LB cases (Table 5). 
Table 4. Correspondence of IGS type with MLS type

\begin{tabular}{|c|c|c|c|c|}
\hline $\begin{array}{l}\text { IGS } \\
\text { type }\end{array}$ & Genospecies & Symptoms & Source & Multi locus sequence type \\
\hline 2 & $\mathrm{AF}$ & EM & human & 1101 \\
\hline 40 & $\mathrm{AF}$ & ACA & human & 1058 \\
\hline 76 & AF & ACA & human & 1033 \\
\hline 92 & AF & ACA, EM & human & 1103,1106 \\
\hline 95 & AF & ACA, EM & $\begin{array}{l}\text { human, } \\
\text { tick }\end{array}$ & $\begin{array}{r}71,1056,1055,1077,1054,168,1081,354,1080 \\
540,1053,1027,1061\end{array}$ \\
\hline 103 & $\mathrm{AF}$ & EM & human & 1096, 1094 \\
\hline 107 & $\mathrm{AF}$ & EM & human & 1052 \\
\hline 108 & AF & EM & human & 1100 \\
\hline 121 & $\mathrm{AF}$ & ACA, EM & $\begin{array}{l}\text { human, } \\
\text { tick }\end{array}$ & $1073,467,204,165,1029,1025,1034,1067$ \\
\hline 122 & $\mathrm{AF}$ & EM & human & 1059 \\
\hline 128 & $\mathrm{AF}$ & ACA, EM & human & $1060,1064,1051,1038$ \\
\hline 133 & AF & EM & human & 1026 \\
\hline 134 & $\mathrm{AF}$ & EM & human & 1031 \\
\hline 135 & AF & EM & $\begin{array}{l}\text { human, } \\
\text { tick }\end{array}$ & 1084, 1093, 1091, 1092 \\
\hline 136 & $\mathrm{AF}$ & EM & human & 462 \\
\hline 151 & $\mathrm{AF}$ & EM & human & 1072 \\
\hline 152 & $\mathrm{AF}$ & ACA, EM & human & 547,335 \\
\hline 156 & $\mathrm{AF}$ & EM & $\begin{array}{l}\text { human, } \\
\text { tick }\end{array}$ & $476,1041,1042,1043$ \\
\hline 157 & AF & $\mathrm{EM}$ & $\begin{array}{l}\text { human, } \\
\text { tick }\end{array}$ & 1076,1066 \\
\hline 158 & $\mathrm{AF}$ & ACA & human & 476 \\
\hline 162 & AF & ACA, EM & human & 72,1044 \\
\hline 171 & AF & ACA, EM & $\begin{array}{l}\text { human, } \\
\text { tick }\end{array}$ & $171,263,479,1071,347,289,1104,1075$ \\
\hline 175 & AF & ACA, EM & $\begin{array}{l}\text { human, } \\
\text { tick }\end{array}$ & $463,171,1090,1088,1089$ \\
\hline 182 & AF & EM & human & 1035 \\
\hline 185 & $\mathrm{AF}$ & EM & human & 1046,351 \\
\hline 141 & BA & EM, NB & human & 84,1021 \\
\hline 143 & BA & EM, NB & human & 84 \\
\hline 47 & $\mathrm{BU}$ & ACA, EM & human & $20,332,1004,21$ \\
\hline 51 & $\mathrm{BU}$ & EM & human & 1003 \\
\hline 9 & GA & EM & human & NA \\
\hline 28 & $\mathrm{GA}$ & EM, NB & human & $86,177,574$ \\
\hline 30 & GA & EM & human & 180 \\
\hline 71 & GA & EM & human & $246,578,1011$ \\
\hline 73 & $\mathrm{GA}$ & EM & human & 187 \\
\hline 74 & GA & & tick & 1006 \\
\hline 12 & $\mathrm{SP}$ & EM & human & 159 \\
\hline
\end{tabular}


There were no IGS STs significantly associated to a particular clinical manifestation of LB.

\section{Predictive value of MLST and IGS genotypes}

Based on the nucleotide sequences of the concatenated MLST genes and IGS, the human (161 samples) and the tick (24 samples) B. burgdorferi s.l. were significantly different $\left(\chi^{2}=23.711, p=0.0084\right)$. The smartpca analysis on this smaller dataset (185 samples for which both MLST and IGS sequences were available) showed that there were 9 SNP's in the MLST sequence that were associated with human cases of LB, while in the IGS sequence there was only one (Table 6a). In order to have a comparable measure for the predictive value of the various genomic regions for pathogenicity of $B$. burgdorferi s.l., we adjusted the number of predictive sites to the length of the DNA sequence $-4788 \mathrm{nt}$ for the concatenated housekeeping genes and $345 \mathrm{nt}$ IGS. Practically, we divided the length of the sequence to the number of predictive sites, obtaining an average of $1 / 532$ predictive sites per $n t$ of the MLST housekeeping genes and of $1 / 345$ predictive sites per nt for IGS.

Table 5. Association of IGS type with source of isolation. The columns "human" and "tick" represent the number of isolates from each of those sources/clinical manifestations corresponding to each of the sequence types. The values in column beta-binomial represent the ps of that statistical test.

\begin{tabular}{lrrr}
\hline IGS type & human & tick & beta-binomial \\
\hline $\mathbf{1 7 1}$ & 27 & 89 & reference \\
$\mathbf{2 8}$ & 14 & 11 & $1.4 \times 10^{-3}$ \\
$\mathbf{7 1}$ & 7 & 4 & $7.1 \times 10^{-3}$ \\
$\mathbf{9 5}$ & 27 & 51 & $4.8 \times 10^{-2}$ \\
$\mathbf{1 2 1}$ & 15 & 23 & $3.6 \times 10^{-2}$ \\
$\mathbf{1 4 1}$ & 9 & 5 & $2.3 \times 10^{-3}$ \\
\hline
\end{tabular}

smartpca analysis based on the SNPs from the concatenated housekeeping genes in the MLST scheme indicated significant differentiation between populations of $B$. burgdorferi s.I. from ACA and EM $\left(\chi^{2}=30.829, p=\right.$ $0.003), \mathrm{NB}$ and EM $\left(\chi^{2}=159.448, p=2.5 \times 10^{-27}\right)$, and ACA and NB $\left(\chi^{2}=260.514\right.$, $\left.p=4.2 \times 10^{-48}\right)$. There were 29 SNPs identified that are capable of significantly differentiating between ACA and NB, 26 SNPs that could differentiate between NB and EM sequences, while only ten SNPs that could differentiate between ACA and EM (Table 6b). 


\section{Discussion}

The present study analysed a set of 775 Borrelia burgdorferi s.l. positive samples to infer relations between their genetic composition and their degrees of pathogenicity in humans. To this end, we used multilocus sequence typing. Previous studies (Hanincova et al., 2013) have shown that this is a tool to be able to discriminate between isolates of $B$. burgdorferi at epidemiological scale.

Borrelia afzelii and B. garinii were the most frequent genospecies in our dataset, which is consistent with the fact that they are the most common genospecies of the B. burgdorferi s.l. complex in Europe (Piesman and Gern, 2004; Rauter and Hartung, 2005). At the opposite pole (with only one MLST profile retrieved from literature) was $B$. bissettii, a genospecies relatively common in North America (Bissett and Hill, 1987; Oliver et al., 2003), but that has been seldom found in Europe (Fingerle et al., 2008; Hulinska et al., 2007; Strle et al., 1997). The low frequency of $B$. spielmanii, and B. lusitaniae could be explained by the low relative abundance and patchy habitats of the competent transmission vertebrates for those genospecies - dormice and lizards (Majlathova et al., 2006; Richter and Matuschka, 2006; Richter et al., 2011). The low frequency of $B$. bavariensis in questing Ixodes ricinus ticks, however, is still awaiting an explanation. The most simple one would be that they are maintained in enzootic cycles with a very narrow vertebrate host spectrum and hosts with low densities. However, $B$. bavariensis seems to be associated with rodents of the genus Apodemus (Hu et al., 1997; Huegli et al., 2002) which are some of the most abundant rodents in Europe (Amori et al., 2008; Schlitter et al., 2008). Alternatively, they could be primarily transmitted by another tick species, being only incidentally bridged over via I. ricinus ticks to humans and other vertebrates via I. ricinus ticks (Gray, 1998). This second hypothesis is sustained also by the low infection rates $-0.7-1.5 \%$ - of questing I. ricinus ticks with B. bavariensis reported by others (Bingsohn et al., 2013; Glatz et al., 2014).

Our MLST analysis indicated a high genetic heterogeneity of $B$. burgdorferi s.l., not only at complex level (with 251 STs in 612 samples) but also within each genospecies. The highest haplotype diversity is found within $B$. afzelii. Our study revealed the existence of 74 new STs of $B$. afzelii, which contribute significantly to the increase of genetic diversity of this genospecies. The low nucleotide diversity of $B$. afzelii (Table 2), together with a high haplotype diversity indicate a high frequency of unique mutations, which could be explained by population expansion. This is consistent with previous findings based on the IGS of $B$. burgdorferi s.l. (Coipan et al., 2013a). A large population size of B. afzelii is not surprising considering that the competent transmission vertebrates for this 


\section{Chapter 6}

genospecies are abundant and easily available (Amori et al., 2008; Hanincova et al., 2003; Schlitter et al., 2008) all over Europe.

Table 6. SNPs differentiating between the B. burgdorferi s.l. populations from different sources and clinical manifestations

a) smartpca results for concatenated sequences of MLST and IGS of human and tick provenience

\begin{tabular}{lr}
\hline gene (position in alignment) & SNP position in alignment \\
\hline clpA (1-579) & 10,297 \\
clpX (580-1203) & 642 \\
nifS (1204-1767) & 1749 \\
pepX (1768-2337) & 1815,2034 \\
pyrG (2338-2943) & 2505,2748 \\
recG (2944-3594) & 3291 \\
IGS (4789-5172) & 4882 \\
\hline
\end{tabular}

b) smartpca results for the MLST housekeeping genes in samples from ACA, EM and NB

\begin{tabular}{|c|c|c|c|}
\hline $\begin{array}{l}\text { gene (position } \\
\text { in alignment) }\end{array}$ & $\begin{array}{r}\text { NB:EM } \\
\text { SNP position in alignment }\end{array}$ & $\begin{array}{r}\text { ACA:EM } \\
\text { SNP } \\
\text { position in } \\
\text { alignment }\end{array}$ & $\begin{array}{r}\text { ACA:NB } \\
\text { SNP position in } \\
\text { alignment }\end{array}$ \\
\hline clpA (1-579) & $12,31,205,349,387,413$ & 12,31 & $\begin{array}{r}12,31,205,349,387, \\
413\end{array}$ \\
\hline $\begin{array}{l}\text { clpX (580- } \\
1203)\end{array}$ & 921,1002 & 1002 & $789,921,1002$ \\
\hline $\begin{array}{l}\text { nifS (1204- } \\
1767)\end{array}$ & $1386,1539,1628$ & 1539 & $1386,1539,1628,1767$ \\
\hline $\begin{array}{l}\text { pepX (1768- } \\
\text { 2337) }\end{array}$ & 2034,2064 & 2034 & 2034,2064 \\
\hline $\begin{array}{l}\text { pyrG (2338- } \\
\text { 2943) }\end{array}$ & $2487,2505,2751,2910,2925$ & 2487 & $\begin{array}{r}2487,2505,2751,2910, \\
2925\end{array}$ \\
\hline $\begin{array}{l}\text { recG(2944- } \\
3594)\end{array}$ & $2983,3110,3192,3312$ & $\begin{array}{r}3110 \\
3312\end{array}$ & $\begin{array}{r}2983,2995,3110,3192, \\
3312\end{array}$ \\
\hline $\begin{array}{l}\text { rplB (3595- } \\
\text { 4218) }\end{array}$ & 4062 & 4062 & 4062 \\
\hline $\begin{array}{l}\text { uvrA (4219- } \\
4788)\end{array}$ & $4431,4618,4656$ & 4656 & $4431,4618,4656$ \\
\hline
\end{tabular}

The lowest haplotype diversity was found for B. bavariensis (Table 2) and indicates a highly clonal genospecies. The low diversity was unlikely to be a 
sampling artefact - the samples came from four countries distributed all across Western and Central Europe - it might be indicative of either fixation of a genotype due to a very small effective population size (Kimura and Ohta, 1969), or a strong selective pressure, such as a very specialized enzootic cycle of this genospecies (Brisson and Dykhuizen, 2004).

The most frequently used measure of risk of human infection with $B$. burgdorferi s.l. is the acarological risk, which is defined as the density of infected ticks (Dister et al., 1997; Glass et al., 1994; Glass et al., 1995; Kitron and Kazmierczak, 1997; Nicholson and Mather, 1996). One of the questions that arises is whether all B. burgdorferi s.l. bacteria have the same infectivity potential or there are bacteria that have a higher intrinsic capacity (given by the genetic makeup) of causing disease. Comparison of haplotype richness and DNA polymorphism in human versus tick-associated bacteria could indicate whether all bacteria found in ticks are equally likely to be infectious for humans. DNA polymorphism analysis showed that the haplotype diversity, nucleotide diversity, and average number of nucleotide differences in $B$. burgdorferi s.l from humans are lower than those from ticks (Table $1 b$ ) for all main genospecies $-B$. afzelii, $B$. garinii, and $B$. burgdorferi. This implies that the population of bacteria causing disease is smaller than the one circulating in questing ticks.

It is, however, known that the sampling limitations could be an impediment for comparing assemblages that contain rare species (Chao et al., 2005; Colwell and Coddington, 1994; Colwell et al., 2004), such as with rare haplotypes of $B$. burgdorferi s.l. To circumvent this drawback we used the rarefaction analysis - a method that is widely used in ecology for comparing species richness of different habitats. The results of our rarefaction analysis showed that the population of bacteria that is capable of causing human disease is only a subset of that from questing ticks (Figure 3 ). This result is therefore not due to various sampling sizes.

\section{Association with human cases of Lyme borreliosis}

While many of the $B$. burgdorferi s.l. spirochetes were found in human cases, some seem to have a higher frequency in human isolates than expected based on their frequency in questing ticks.

To test our hypothesis of differential infectivity of B. burgdorferi s.l., we compared the frequencies of the different genospecies of Borrelia in two sources - human and tick. The two species that were significantly more frequent in human cases than in questing ticks were $B$. afzelii and B. bavariensis (Table 3a) both mammal-associated Borrelia. Borrelia lusitaniae and $B$. valaisiana were, as expected, negatively associated with LB. The association of $B$. afzelii with human 


\section{Chapter 6}

cases could be due to their ability to cause a long lasting and more prominent $\mathrm{EM}$, as it was shown in previous studies (van Dam et al., 1993), being, therefore, easier to detect. In the case of $B$. bavariensis, the strikingly low frequency in questing ticks and high frequency in LB patients (Table 3a) could be explained by higher infectivity of these bacteria. Although, both $B$. garinii and B. burgdorferi s.s. comprised STs that were only isolated from LB patients, there was no significant association of these genospecies with the human cases. One possible explanation is the lower sample size available for these genospecies, comparing with B. afzelii; additional sampling of these genospecies might lead, in future studies, to clarification of the matter of differential infectivity of these spirochetes.

We hypothesize that the reason for which mammal associated Borrelia are significantly more often retrieved from humans than bird associated Borrelia is that humans are also mammals and the factors that trigger the specificity of Borrelia for small rodents (e.g. outer surface protein B, as suggested by Vollmer et al. (2013)) could be the same ones that are responsible for facilitating the establishment of localized infection with these bacteria in humans. This would make the transmission of the bacteria more facile between vertebrates of the same class (i.e. mammals) than between vertebrates of different classes (i.e. birds and mammals). The imbalanced clustering of pathogenic $B$. garinii could be an indication of an ongoing evolution process that might lead to the acquisition of the required genetic elements for infectivity in mammals. Such an event could have led to the emergence of $B$. bavariensis.

Our findings that not all genospecies, clusters, or STs are equally likely to cause disease in humans suggest that the spirochetes of $B$. burgdorferi s.l. have different infectivity properties, not only between but also within the genospecies, and this has direct implications on the epidemiology and risk assessment of human infections with these bacteria. While previous studies have showed the propensity of some STs of $B$. afzelii and B. burgdorferi to cause LB (Hanincova et al., 2013; Jungnick et al., 2015), our analysis indicates that at European scale the genetic diversity of Borrelia in humans is much higher than previously acknowledged, with 68 B. afzelii STs, of which 47 are newly described.

\section{Association with disseminated/persistent forms of Lyme borreliosis}

Our results indicated that not all spirochetes are equally invasive/persistent, with some being more likely to cause ACA and others NB. It seems that the success rate of culturing $B$. burgdorferi s.l. is higher from EM than from NB (Kuiper et al., 1994; van Dam et al., 1993). However, it could be that a large fraction of the patients with EM, particularly with mild rashes - such as it 
was previously reported for B. garinii and B. bavariensis (van Dam et al., 1993) do not get to the general practitioners and dermatologists to have the bacteria cultured. On the other hand, it is very likely that the patients with severe neurological manifestations will often undergo thorough clinical examinations, with ensuing culturing of the spirochetes. Consequently, some of the bacteria causing NB could be overlooked in the EM cases. Future studies, on larger datasets, can establish whether these associations represent real invasive capacities of the bacteria or if they are the result of undersampling in EM cases and better sampling in ACA or NB.

The low number of samples with LA causing bacteria in our dataset, due to difficulties in culturing Borrelia from LA patients, did not allow us to gain any results and draw any meaningful conclusions for these. Sampling with PCR-based techniques from a wider range of LB clinical manifestations, will most probably shed more light on the association of the bacterial genotype and the pathogenic properties of these bacteria.

One of the possible reasons for lack of association between the chromosomal DNA profile of the bacteria and their differential pathogenicity is recombination. Recombination is known to maintain sequence polymorphisms within B. burgdorferi s.l. populations (Haven et al., 2011) and the high number of STs can impede the identification of associations with clinical manifestations of LB. The test for recombination (not shown) indicated that this is a frequent event even for chromosomal housekeeping genes, as other studies (Haven et al., 2011) have also revealed.

An alternative explanation for imperfect association of genotype with infectiousness and invasiveness is the synergism between some bacteria. It has been previously shown that $B$. burgdorferi s.l. coexists in ticks with some other tick-borne pathogens (Belongia, 2002; Burri et al., 2011; Coipan et al., 2013b; Ginsberg, 2008; Koetsveld et al., 2015; Lommano et al., 2012; Nieto and Foley, 2009; Reye et al., 2010). Likewise, it is known that the severity of LB may be affected by simultaneous infections with other bacteria, such as Anaplasma phagocytophilum (Belongia, 2002; Holden et al., 2005; Swanson et al., 2006; Thomas et al., 2001), that are capable to modulate host immunity and, therefore, increase susceptibility to various second pathogens. Thus, coinfection might be partly responsible for the variability in clinical manifestations that are usually associated with LB.

\section{IGS vs. MLS typing}

When comparing the predictive values of the chromosomal markers used in this study for infectivity of $B$. burgdorferi s.l., our study suggests that IGS could 


\section{Chapter 6}

perform just as good as the housekeeping genes in the MLST scheme. The fact that a shorter DNA sequence could be just as informative as MLST could be of use in the process of rapid typing of bacteria for clinical purposes, but also in first stage epidemiological screening. It remains to be seen if this association still holds for larger datasets.

When comparing the predictive values of IGS and MLST for dissemination/persistence of $B$. burgdorferi s.l., it was obvious that IGS could not be associated with any clinical manifestations of LB, while MLST could indicate differential invasiveness for the various STs. The reason for this could be that the chance of a mutational event is higher with a longer DNA sequence - such as the concatenated housekeeping genes - and there are, therefore, more nucleotides that can differentiate between the disseminated forms of LB.

In conclusion, comparison of MLST profiles of $B$. burgdorferi s.l. strains isolated from LB patients with those of questing ticks suggests the existence of $B$. burgdorferi STs with differential pathogenic properties in humans. The infectivity seems to be influenced by the vertebrate specificity of the bacteria, with mammal-associated bacterial STs being more frequently retrieved from human cases than the bird-associated ones. The invasive potential of the different STs appears to be a function of the genospecies, with $B$. bavariensis and $B$. garinii causing neuroborreliosis, while $B$. afzelii can cause persistent skin infection in the form of acrodermatitis chronica atrophicans. The existence of specific STs with a higher predilection for late/disseminated forms of LB could be beneficial in timely typing of the bacteria in an infected patient and, therefore, in improving the prognosis for the evolution of infection and allowing for differential treatment and monitoring. Imperfect associations indicate, however, the existence of other factors that play a role in infectiousness and invasiveness, and require more studies on the genetic diversity of the LB spirochetes isolated from human samples.

\section{Acknowledgements}

We are grateful to lan Livey for help in collecting the material used in this study. We thank Arno Swart for advice on the statistical analysis and for the stimulating discussions. We give thanks to Sipke van Wieren and Willem Takken for fruitful discussions on the study design and the results of our analysis. This study was financially supported by the Ministry of Health, Welfare and Sport (VWS). 


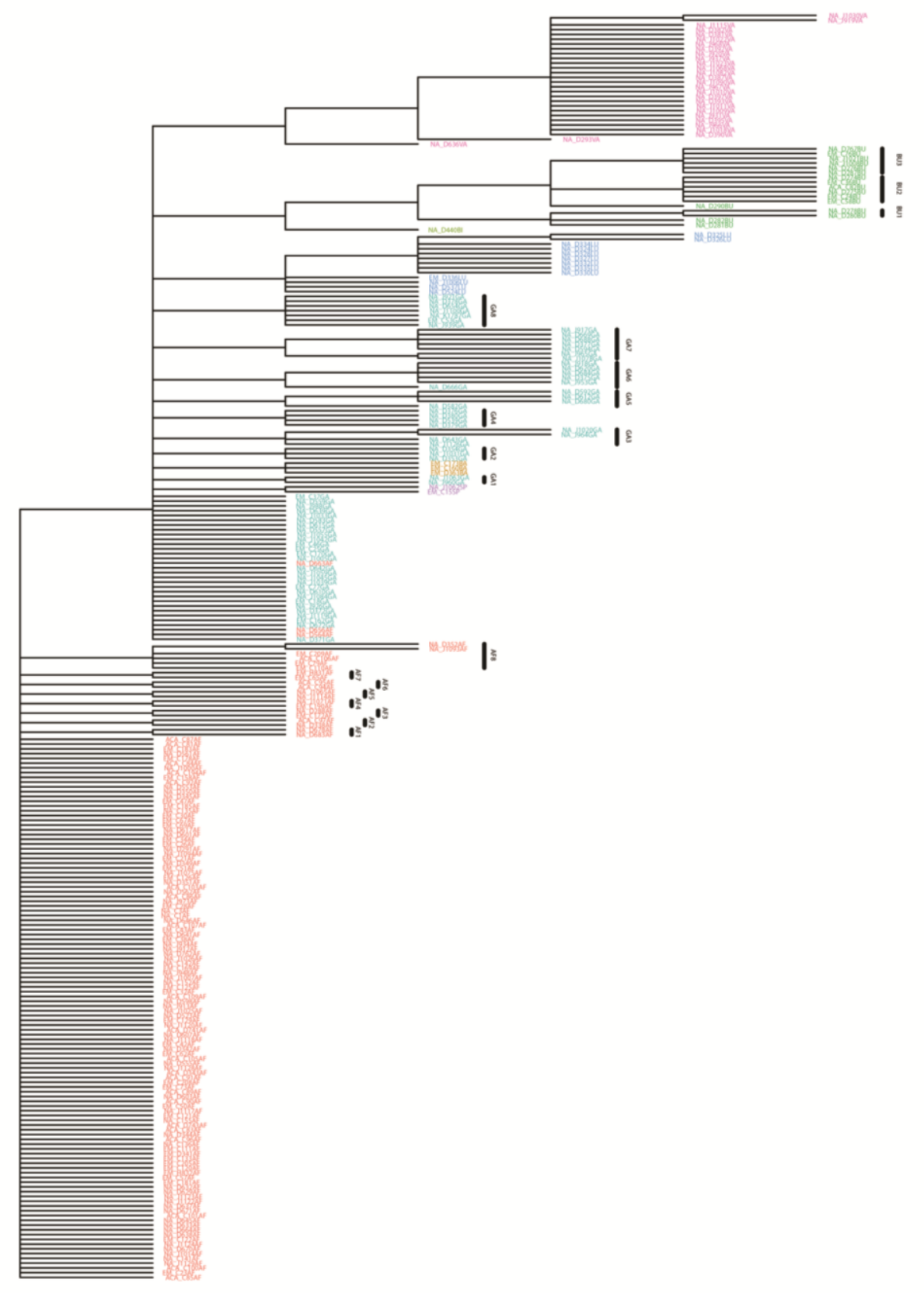

Supplementary Figure 1. Collapsed MLST tree of isolates; vertical thick lines delineate the clusters obtained by collapsing branches with more than $90 \%$ bootstrap support in Biopython. 

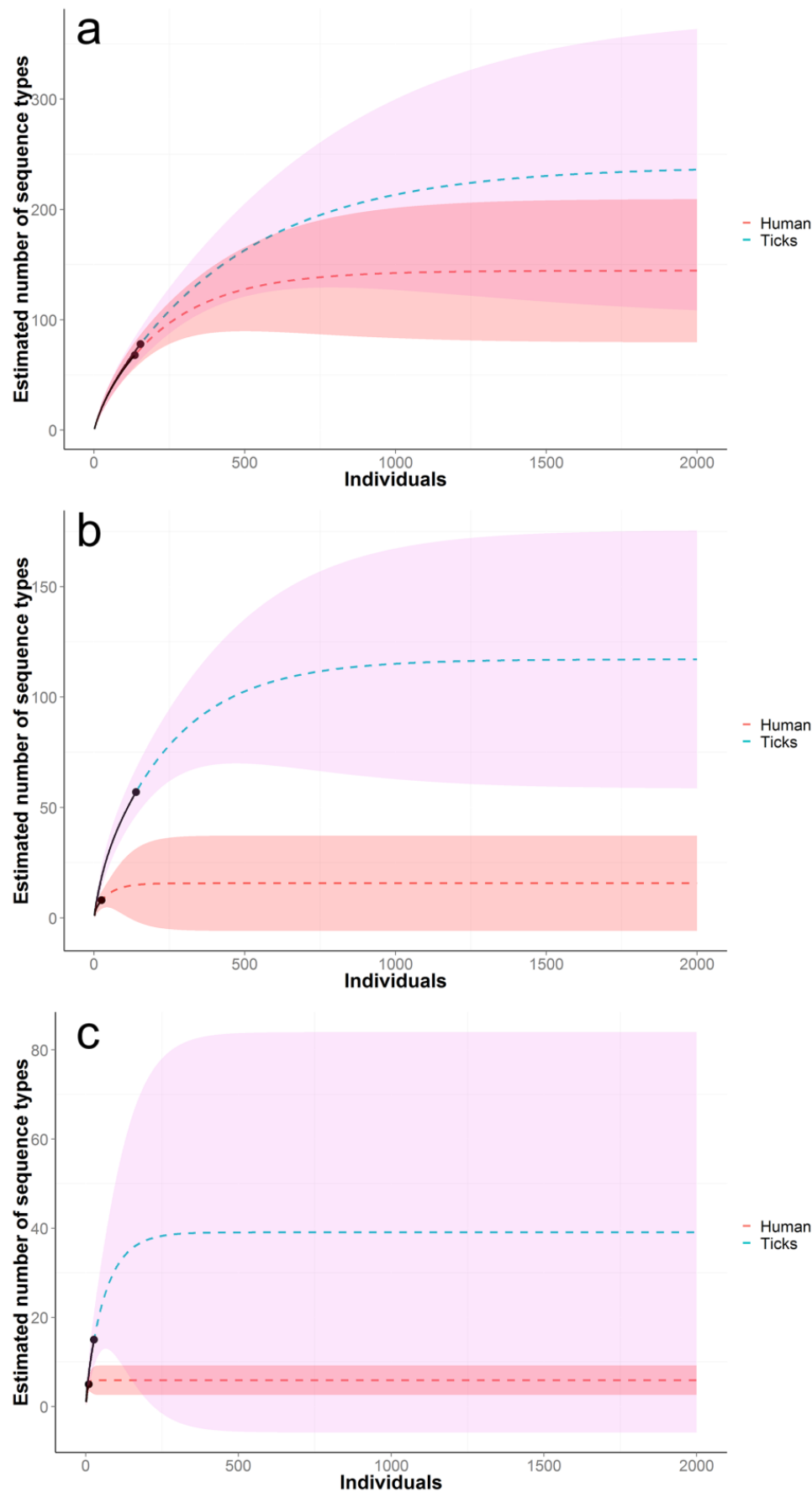

Supplementary Figure 2. Rarefaction analysis for a) B. afzelii, b) B. garinii, and c) B. burgdorferi s.s 


\section{Chapter 7}

\section{General Discussion}

\section{Graphical abstract}

The major components of the enzootic cycle of Borrelia burgdorferi s.I. in Europe. The various developmental stages of the ticks feed on various classes of vertebrate hosts. The competence of the vertebrates for the $B$. burgdorferi s.l. genospecies determines what bacteria will be taken up by the ticks feeding on them. The distribution of ticks on the hosts in combination with the density of hosts determines the prevalence of the bacteria in the next stage of the ticks. For simplicity insectivores are also included under the name of rodents. Bacteria that have been shown to cause disease in humans are marked by the dark-blue frame.

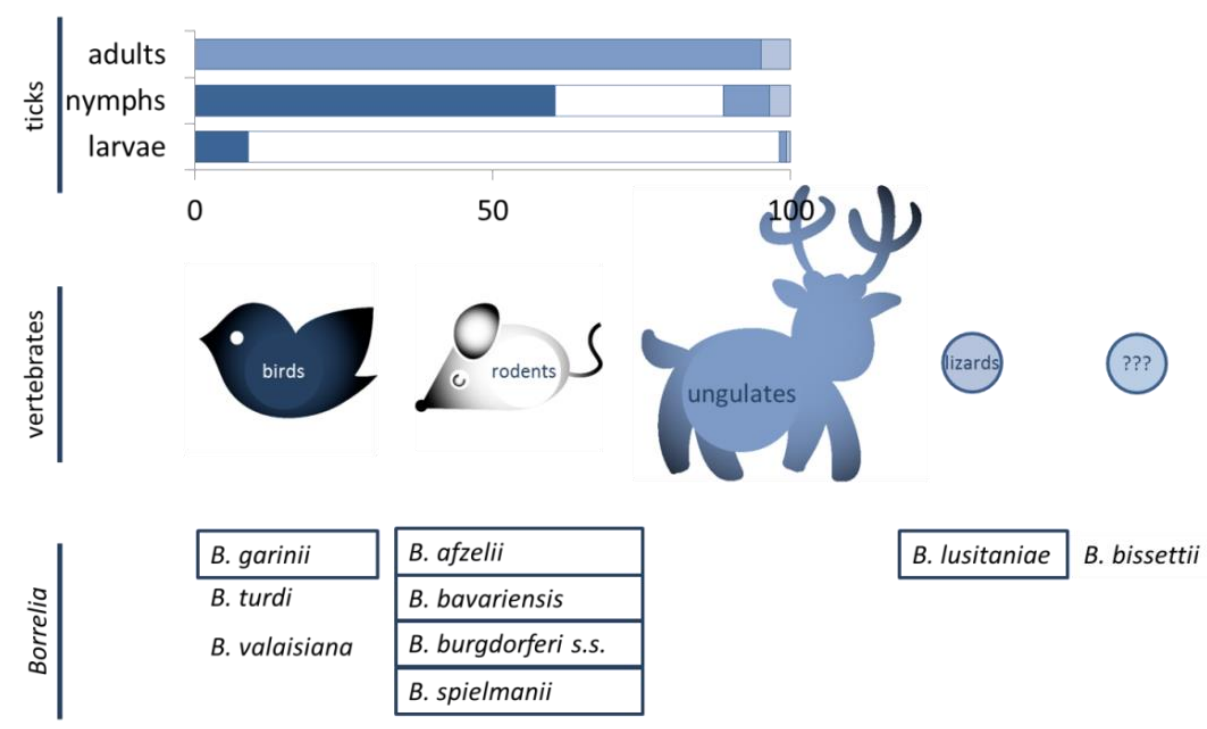




\section{Chapter 7}

Lyme borreliosis is the most prevalent vector-borne disease in the temperate regions of the Northern hemisphere. The bacteria that cause it are members of the Borrelia burgdorferi sensu lato complex, a group of spirochaetes that are transmitted by hard ticks of the Ixodes ricinus complex. In this thesis, I explored aspects of the ecology and molecular adaptations of $B$. burgdorferi s.l. at various scales, from complex to genospecies level. In this chapter, I will discuss to what extent the research approach answered the research questions described in the introduction (Chapter 1), how do the findings complement preexisting knowledge in the field and which are the consequences for public health. As, in every field of knowledge, every answer generates more questions, I will also highlight questions that might be worthwhile approaching in future research.

\section{Genetic diversity in B. burgdorferi s.l.}

The ecological adaptations of $B$. burgdorferi s.l. are underpinned by a complex genomic structure and gene expression. The genome of these spirochaetes is highly fragmented, with, next to the chromosome, up to 21 different plasmid types (Casjens et al. 2011,Fraser et al. 1997, Schutzer et al. 2011,Schutzer et al. 2012). This is the greatest number of genomic elements ever found to coexist within a bacterial cell. The linear chromosome contains the core house-keeping genes, with a summed length of approximately $950 \mathrm{kbp}$ (Fraser et al. 1997). The plasmids contain the majority of the lipoproteins genes (Barbour 1988, Casjens et al. 2012) that are essential for transmission between vertebrates and ticks and are differentially expressed in the various phases of the enzootic cycle (Schwan and Piesman 2000). The high fragmentation of the genome is considered to be a facilitating element in the multiple niche shifts that a spirochete has to undergo. However complex the genome of $B$. burgdorferi s.l. may be, it misses many of the essential house-keeping genes, which is what makes it an obligate parasite, having to use the vertebrate host as well as the tick host for survival (Posey and Gherardini 2000,Purser et al. 2003).

There is large genetic variation between the genospecies. Recent wholegenome studies have revealed that these genetic differences consist mainly in plasmid content and gene location on the various plasmids (Casjens et al. 2011,Casjens et al. 2012,Schutzer et al. 2011,Schutzer et al. 2012). The content of the linear plasmids may be shuffled by telomere fusion (Kobryn and Chaconas 2005) but the repertoire of genes remains relatively consistent (Casjens et al. 2012).

Beside the differences among the various genospecies in the $B$. burgdorferi s.l. complex, there are also marked differences within the 
genospecies. These have been investigated both at chromosomal and plasmid level, and appear to span over house-keeping as well as virulence-encoding genes. Furthermore, chromosomal and plasmid genes have been found in linkage disequilibrium (Bunikis et al. 2004), which is unexpected for genetic elements that are not physically linked.

The gold standard for genotyping of B. burgdorferi s.l. nowadays is multilocus sequence typing (MLST), based on eight housekeeping genes on the chromosome, which undergo slow evolution and show nearly neutral variation (Margos et al. 2008,Urwin and Maiden 2003). Furthermore, MLST has revealed geographical structuring of $B$. burgdorferi s.l. populations (Vitorino et al. 2008,Vollmer et al. 2011). Our studies have shown that the 5S-23S rDNA intergenic spacer (IGS) is also a marker that can discriminate between the genospecies of $B$. burgdorferi s.l. and detect genetic differentiation between the bacteria of various geographic proveniences (Coipan et al. 2013b), while having a comparable predictive value of human pathogenic B. burgdorferi s.l. to that of MLST analysis (Coipan et al. 2016) (Chapter 3, Chapter 6). Therefore, in the studies presented here we have used IGS as a marker for both genospecies determination and discrimination at intra-genospecies level, between the various Borrelia genotypes.

Which are the main vertebrate hosts of Borrelia burgdorferi s.l. in Europe?

At large geographical scale the distribution of the various Borrelia genospecies is primarily driven by the vertebrate host they are adapted to (Kurtenbach et al. 2006b,Vollmer et al. 2011), with avian-associated Borrelia having a wider areal than rodent-associated ones. Thus, avian-associated Borrelia, such as B. garinii, B. turdi, and B. valaisiana, are spread over both Europe and Asia. The genospecies that are mammal-associated, such as $B$. spielmanii, $B$. yangtze, and B. tanukii, seem to be confined to the geographic restrictions of their vertebrate hosts (Fukunaga et al. 1996, Margos et al. 2015,Margos et al. 2011,Richter et al. 2004). The reason that B. afzelii and $B$. bavariensis are spread across all of Eurasia (Margos et al. 2013,Rauter and Hartung 2005), even though they are both associated with rodents, could be their broad host range, with multiple rodent species serving them as reservoirs (Chapter 2). Host specificity could also be the reason that some of the genospecies remain confined to certain geographical areas, where the competent hosts are most abundant. This is the case of Borrelia yangtzensis or $B$. tanukii in Asia, which are amplified by rodents of the species Suncus murinus and Mus caroli (Kawabata et al. 2013,Margos et al. 2015), and Myodes rufocanus, Myodes smithii and Apodemus speciosus (Masuzawa et al. 1996b), respectively. 


\section{Chapter 7}

In areas where the specific vertebrate hosts are absent or less abundant the genospecies cannot persist or, if they do, it is at very low abundance levels. However, many of the genospecies that were once thought to have a relatively limited distribution area (e.g. B. turdi or B. bavariensis), have later been proven to be widespread (Margos et al. 2013,Norte et al. 2015). In some cases it could be a matter of recent introduction of the genospecies by means of migratory birds (Hasle et al. 2011). For B. bavariensis, recent whole genome studies have shown that the European strains are almost clonal, while in the Asian strains there is a higher genetic diversity. This could be the result of a recent introduction of the genospecies in the European landscape by a shift in the vector tick species - from only I. persulcatus to I. ricinus as a second arthropod host (Gatzmann et al. 2015). It is equally possible that the earlier failure to detect these genospecies was the result of methodological limitations. This kind of questions will probably be answered to by use of phylogeography and population genetics studies.

Previous studies have identified more than 100 vertebrate species (mammals, birds and lizards) that can act as transmission and/or amplification hosts for B. burgdorferi s.I. (Piesman and Gern 2004a). Ungulates are some of the few vertebrates that are incompetent Borrelia transmitters, and this seems to be due to the inability of Borrelia to circumvent the innate immune response of ungulates (Kurtenbach et al. 2002a).

The success of transmission and maintenance of $B$. burgdorferi s.l. in enzootic cycles depends on the density and abundance of the various vertebrate host species. In Chapter 2 we have estimated the relative importance of vertebrate species that are abundant in European forests for maintenance of $B$. burgdorferi s.l. as well as their realized reservoir competence, i.e. the proportion of blood fed larvae that become infected with B. burgdorferi s.l. (LoGiudice et al. 2003). Among small mammals, Eliomys quercinus, Microtus agrestis, and Sorex araneus have the highest realized reservoir capacity. It is, however, Apodemus sylvaticus and Myodes glareolus that have the highest relative importance for infecting larvae with $B$. burgdorferi s.l.; that is due to their high densities and relatively high larval burdens. The second most important group for $B$. burgdorferi s.l. maintenance is that of thrushes (Turdus merula and Turdus philomelos), which have intermediate densities and larval burdens, but a very high realized reservoir competence. This indicates that the number of larvae feeding on a host species and its density are more important than the realized reservoir competence of that host species in determining the contribution of a host species to infect larvae. Furthermore, it suggests that the prevalence of the two main B. burgdorferi s.l. genospecies in questing ticks is mainly dependent on the distribution of larvae over rodents and thrushes. 
I suggest that there are at least two distinct mechanisms behind the maintenance of small mammal-transmitted and bird-transmitted Borrelia spp. (Chapter 2)

1) Because small mammals have low nymphal burdens (Gassner et al. 2013,Hofmeester et al. 2016), their infection prevalence with B. burgdorferi s.l. is relatively low. However, because they feed a large proportion of the larvae, even a small infection prevalence of the host species can result in a high density of infected nymphs with small mammal-transmitted Borrelia spp. like B. afzelii. This high density of larvae infected with small mammal-transmitted Borrelia spp. results in a sufficiently-large number of infected nymphs to, in turn, infect small mammals in spite of their low nymphal burdens. Furthermore, the life cycle of $I$. ricinus takes 2-6 years to complete, with each life cycle stage (larva, nymph and adult) taking one year or even more (Gray 1991). Small rodents, on the other hand, are short-lived, with few adults surviving from one summer to the next in the wild (Ostfeld 1985). Thus, the infected larvae that will moult into infected nymphs can infect a couple of generations of rodents.

2) Avian-transmitted Borrelia spp., like B. garinii and B. valaisiana, on the other hand, seem to be dependent on a high infection prevalence of their host species due to relatively high nymphal burdens (Chapter 2). Therefore, even with a low larval burden and intermediate host density, sufficient numbers of infected nymphs are produced to infect birds, which completes the maintenance cycle for avian-transmitted Borrelia spp. However, this strategy is probably not only restricted to avian-transmitted Borrelia spp. Borrelia spielmanii is a candidate for a similar maintenance strategy in mammals as it is often found with low prevalence in questing ticks, but with high prevalence in one of its principal hosts, Eliomys quercinus and Erinaceus europaeus (Richter et al. 2004). These differences in maintenance strategies could indicate that less common Borrelia spp., or other tick-borne pathogens with low infection prevalence in questing nymphs, might be maintained by host species with high nymphal or adult burdens (Ostfeld et al. 2014). e.g. one would expect that B. garinii will be more abundant in questing adults than in questing nymphs. Comparative studies on the infection prevalence of the various stages of $I$. ricinus with $B$. burgdorferi s.l. genospecies or other tick-borne pathogens could test this hypothesis.

These alternative transmission strategies suggest that different $B$. burgdorferi s.l. genospecies have specialized either on host species that occur in high densities, or on host species that feed large numbers of ticks, with the exception of larger bodied mammalian species such as deer. 


\section{Chapter 7}

The host community has been hypothesized to generate the intraspecific genetic diversity of $B$. burgdorferi s.l. by various mechanisms. One of them is the multiple niche polymorphism balancing selection that implies that various hosts can act as ecological niche for a subset of the strains of a species (Gliddon and Strobeck 1975,Levene 1953). Such host specialization of the Borrelia burgdorferi s.l. strains has been described especially for B. burgdorferi s.s. in North America, based on the outer surface protein $C$ gene (ospC) (Brisson and Dykhuizen 2004) and multilocus sequence typing (MLST) (Mechai et al. 2016). Also European studies reported differentiation among the strains of $B$. afzelii isolated from various rodents, based on ospC and ribosomal protein $\mathrm{L} 2$ gene (rplB) (Jacquot et al. 2014). The second mechanism that could maintain the genetic diversity of Borrelia burgdorferi s.l. at some loci is the negative frequency dependent polymorphism. This postulates that no strain has a maximum fit within a certain host species but that initial infection of a host triggers an immune response that will be protective against subsequent infections with genetically similar bacterial strains (Barthold 1999,Gromko 1977). Thus, the strain that is most abundant at some point in time will gradually decrease in frequency by negative selection from the host, favouring another one to become more frequent; a temporal shift in the frequency of the various strains occurs in this manner. This theory has also been supported by the results of some European studies (Durand et al. 2015, Hellgren et al. 2011). The results in Chapter 4 support the second hypothesis, in the sense that bacterial haplotypes for ospC (one of the strongest elicitor of the vertebrates' immune response to Borrelia burgdorferi s.l.) are found to have variable frequencies in different geographical areas while the local assemblage of haplotypes seems to reflect the large-scale assemblage. Thus, while in Switzerland the most abundant ospC type in a study by Durand et al. (2015) was A10, followed at more than $30 \%$ difference by $A 9$, in our study $A 9$ and $\mathrm{A} 10$ came in the 3rd and 4th positions. This could be the reflection of a negative frequency-dependent selection mechanism, which allows for fluctuations in time of the alleles' frequencies and consequently for the shift in frequencies at different geographic locations.

Another observation in favour of the negative-frequency dependent selection is the existence of a high degree of linkage disequilibrium between the alleles at loci on the chromosome and plasmids (Bunikis et al. 2004). Thus, in spite of the fragmented genome of $B$. afzelii, and subsequent facility for gene exchange, the horizontal gene transfer is not a pervasive phenomenon in these bacteria. That could be another indication that these spirochetes have evolved to have equal fitness for both species of the main vertebrate hosts. Given the frequency of double/multiple Borrelia infections observed in the larvae feeding on rodents, there would be plenty of opportunities for lateral gene transfer, 
should one of the genotypes have an advantage in resisting the host's immune response. This implies that the innate immune response of the various small rodents does not exempt a strong selective pressure among the genotypes of $B$. afzelii.

\section{What is the composition of B. burgdorferi s.I. in questing I. ricinus ticks?}

Ixodes ricinus is the main vector of B. burgdorferi s.l. in Europe (Gern and Humair 2002b). Although their contribution to the maintenance of the spirochaetes seems to be lower, in certain habitats Ixodes hexagonus and Ixodes uriae can also transmit B. burgdorferi s.l. (Gern et al. 1997,Olsen et al. 1993). e.g. Ixodes hexagonus could be responsible for the enzootic cycle/maintenance of Borrelia bavariensis, with occasional spill-over to I. ricinus.

Borrelia afzelii was the most prevalent genospecies in the questing $I$. ricinus nymphs in The Netherlands, as well in our study (Chapter 5 ) as in previous ones (Wielinga et al., 2006; Gassner et al., 2011). Furthermore, B. afzelii seems to be the most common genospecies in most areas of Europe (Rauter and Hartung 2005,Ruyts et al. 2016). The feeding pattern of ticks could explain the predominance of $B$. afzelii. We found that $89 \%$ of the infected larvae had fed on rodents. This should result in a large percentage of $B$. afzelii-infected nymphs as (these) rodents transmit B. afzelii (Hanincova et al. 2003a). Thrushes fed only $10 \%$ of the infected larvae (Chapter 2 ), which could explain the relatively low percentages of $B$. garinii and B. valaisiana that we (Chapter 5 ) and others (Gassner et al. 2011,Ruyts et al. 2016) found in questing infected nymphs (Figure 1).

It could be that the specialization of $B$. burgdorferi s.l. genospecies is partly influenced by the tick feeding behaviour. Evolutionary theory predicts that specialist pathogens are favoured if their hosts are abundant, whereas generalists would do better when the encounters with host species are less predictable (Woolhouse et al. 2001). In this context, the larvae of I. ricinus, that are heterogeneously distributed, have a higher encounter rate with small mammals that are highly abundant and very actively foraging in the leaf litter (Mejlon 1997). Thus, small mammals, occurring in high densities and having relatively high larval burdens, represent the most important host group for feeding I. ricinus larvae. The nymphs, in turn, which have a more homogeneous distribution, have more comparable chances of encountering either a rodent or a bird. Therefore, nymphs are almost evenly distributed on rodents and birds (Chapter 2). 

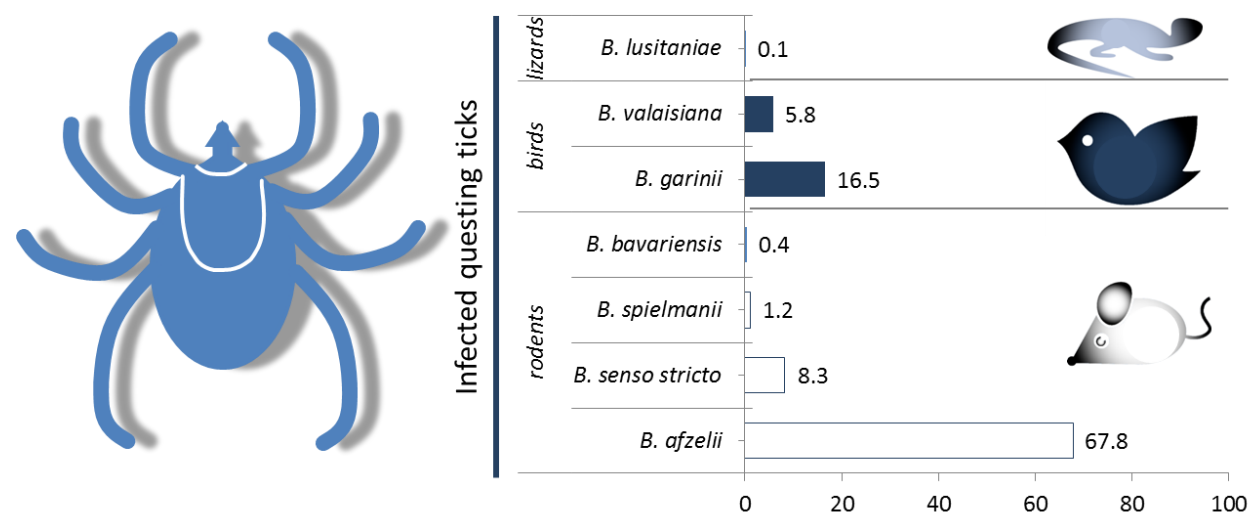

Figure 1. Prevalence of infection with the various B. burgdorferi s.l. genospecies of questing $I$. ricinus nymphs. All prevalences add up to $100 \%$.

Tick density affects not only the transmission efficiency of $B$. burgdorferi s.l., but also the genetic diversity of these bacteria. It is known that the larger the population, the higher the genetic diversity. This is especially true for genetic markers that are neutrally evolving, such as the 5S-23S rDNA intergenic spacer (IGS). Preliminary data from a study on Borrelia burgdorferi s.l. in 20 different locations in The Netherlands, suggests that the haplotype diversity of IGS in $B$. afzelii correlates with the density of ticks infected with this genospecies (Coipan et al., unpublished). Similarly, analysis of MLST data showed that there is a higher haplotype diversity within B. afzelii compared to B. garinii, while the genetic differentiation is, by contrast higher in the latter. These results are consistent with a larger population size of $B$. afzelii, which in turn is consistent with a higher density of ticks infected with $B$. afzelii than of those infected with $B$. garinii. The higher genetic differentiation within $B$. garinii reflects, also, the lower contact rate of the ticks with the birds when compared with the mammals, allowing thus for evolution of distinct lineages of B. garinii (Chapter 6).

Coinfection with other microorganisms may facilitate or impair the transmission efficiency of the Borrelia. These coinfections seem to not represent an exception but are more likely the rule. We found that $6.3 \%(350 / 5570)$ of the questing ticks in The Netherlands are infected with more than one pathogen of different genera. A significant negative association was found between $B$. afzelii and Rickettsia helvetica, as well as between Neoehrlichia mikurensis and $R$. helvetica. On the other hand, significant positive associations were found between B. afzelii and N. mikurensis and between Borrelia and Babesia spp. (Chapter 5). These findings, together with a seasonal synchrony of the infection prevalences with these pathogens in questing ticks indicate that B. afzelii, $N$. 
mikurensis, and Babesia share the same reservoir hosts, while R. helvetica is maintained in other enzootic cycles, probably by birds (Heylen et al. 2016). Multiple studies have reported coinfection in questing ticks with several of the tick-borne pathogens (Belongia 2002,Burri et al. 2011,Ginsberg 2008,Lommano et al. 2012,Nieto and Foley 2009, Reye et al. 2010). Others have reported serological evidence of coinfection with spotted fever group rickettsiae and $B$. burgdorferi s.l. in patients suspected of Lyme neuroborreliosis (Koetsveld et al. 2016). It is possible that the severity of Lyme disease is affected by simultaneous infections with other tick-borne pathogens (Belongia 2002,Swanson et al. 2006). Some of them, such as $A$. phagocytophilum, modulate host immunity and increase susceptibility to various second pathogens, including $B$. burgdorferi s.l. (Holden et al. 2005, Thomas et al. 2001). Others, such as Rickettsia spp., infect endothelial cells, which form the basic layer of the blood brain barrier, rendering this temporarily permeable to B. burgdorferi s.l. (Koetsveld et al. 2016). Thus, coinfection might be partly responsible for the transmission efficiency of $B$. burgdorferi s.l. between hosts and ticks but also for the variability in clinical manifestations that are usually associated with Lyme borreliosis.

\section{What is the composition of $B$. burgdorferi s.l. in human Lyme borreliosis?}

The most frequently Borrelia genospecies retrieved from human cases of Lyme borreliosis are B. afzelii, B. garinii, B. burgdorferi s.s., and B. bavariensis (Stanek et al. 2012). The genetic differences between the genospecies seem to affect not only their enzootic associations but also the progress of human infection with Borrelia (Stanek et al. 2012). The results of the study described in Chapter 6 confirmed that $B$. afzelii is mostly associated with erythema migrans (EM) and acrodermatitis chronica atrophicans (ACA), while B. garinii infections can lead to neurological symptoms (i.e. neuroborreliosis, NB) (Stanek et al. 2012) (Figure 2).

The public health implications of multiple strains and lineages within a genospecies of Borrelia have been investigated in several studies. From a public health perspective, it is important to be able to differentiate between the infectious and non-infectious Borrelia spirochaetes or between the invasive and non-invasive ones. Discriminating between these types is helpful for disease risk assessment and management.

Research on B. burgdorferi s.s. in North America has shown that some major sequence types of the ospC and certain sequence types of 16S-23S rRNA intergenic spacer are more frequently found in disseminated cases of LB (Dykhuizen et al. 2008,Strle et al. 2011,Wormser et al. 2008). More recently, Hanincova et al. (2013) have used MLST on eight housekeeping genes on the 
chromosome, which undergo slow evolution and show nearly neutral variation (Margos et al. 2008,Urwin and Maiden 2003), to investigate these associations. They have shown significant associations between clusters of sequence types (clonal complexes) of $B$. burgdorferi s.s. and localized or disseminated forms of LB. It seems, thus, that the genetic makeup of the pathogenic spirochaetes is one of the determinants for the symptomatology they cause.

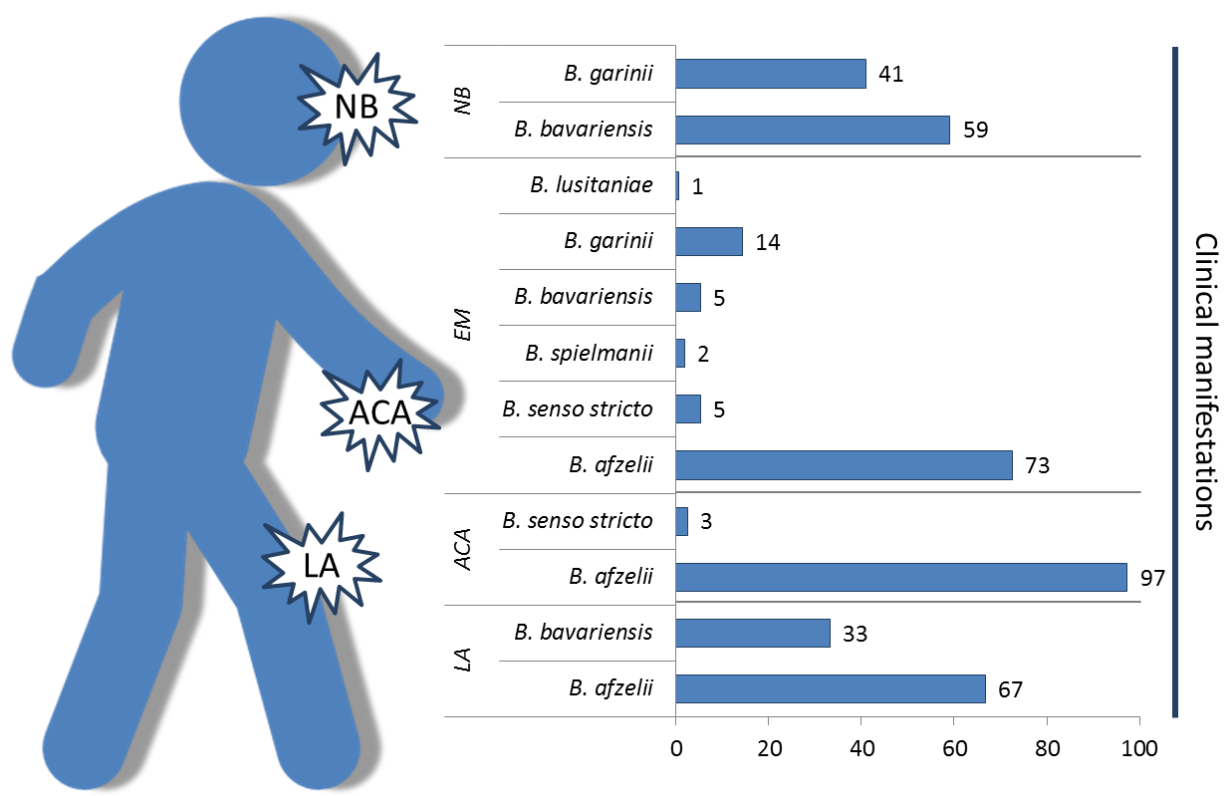

Figure 2. Localization of human clinical manifestations of Lyme borreliosis (NB = neuroborreliosis, $\mathrm{EM}=$ erythema migrans, $\mathrm{ACA}=$ acrodermatitis chronica atrophicans, LA $=$ Lyme arthritis) and prevalence of various $B$. burgdorferi s.l. genospecies in each manifestation. EM does not have a preferential localization - it occurs at the site of the tick bite. Prevalences within a manifestation add up to $100 \%$.

Using European isolates of Borrelia burgdorferi s.l. and tick lysates positive for Borrelia burgdorferi s.l., we have shown that also within the European genospecies $-B$. afzelii, $B$. bavariensis, and $B$. garinii, there are sequence types that are more often associated with human cases of Lyme borreliosis than expected based on their frequency in questing ticks (Chapter 6 ). The two species that were significantly more frequent in human cases than in questing ticks were B. afzelii and B. bavariensis - both mammal-associated Borrelia. Borrelia lusitaniae and B. valaisiana were, as expected, negatively associated with LB. The association of $B$. afzelii with human cases could be due to their ability to cause a long lasting and more prominent EM, as it was shown in previous studies 
(van Dam et al. 1993), being therefore, easier to detect. In the case of $B$. bavariensis, the strikingly low frequency in questing ticks and high frequency in LB patients could be explained by higher infectivity of these bacteria. Remarkably, despite its high incidence in ticks and erythema migrans, in terms of disease burden (as measured by disability-adjusted life year), B. afzelii is probably of least concern. Most of the EMs disappear after antibiotic treatment and the relatively rare late manifestations of infections with this bacterium pertain to skin alterations (Acrodermatis Chronica Atrophicans). On the other hand, the low incidence of infections with B. bavariensis and B. garinii leads more often to severe late clinical manifestations, such as neuroborreliosis, which in terms of disease burden, probability to develop long-term sequella and public health impact, is a (far) more severe disease than erythema migrans.

We observed that mammal-associated Borrelia genospecies, such as $B$. afzelii, $B$. bavariensis, and $B$. spielmanii, are more often isolated from patients than avian-associated Borrelia genospecies (B. garinii and B. valaisiana) (Chapter 6). We hypothesize that the reason for which mammal-associated Borrelia are significantly more often retrieved from humans than bird-associated Borrelia is that humans are also mammals and the factors that trigger the specificity of Borrelia for small rodents (e.g. outer surface protein $\mathrm{B}$, as suggested by Vollmer et al. (2013)) could be the same ones that are responsible for facilitating the establishment of localized infection with these bacteria in humans. This would make the transmission of the bacteria easier between vertebrates of the same class (i.e. mammals) than between vertebrates of different classes (i.e. birds and mammals).

The finding that not all genospecies, clusters, or STs are equally likely to cause disease in humans suggests that the spirochaetes of B. burgdorferi s.l. have different infection properties, not only between but also within the genospecies, and this has direct implications for the epidemiology and risk assessment of human infections with these bacteria.

\section{What are the implications for public health?}

Although bites of infected larvae and adult ticks can cause LB as well, the infected nymphs are considered as constituting the main source of human infection with Borrelia burgdorferi s.l., simply due to their abundance. Therefore, the acarological risk of human infection with Borrelia burgdorferi s.l. has been defined as the density of infected questing nymphs (Dister et al. 1997, Glass et al. 1994,Glass et al. 1995,Kitron and Kazmierczak 1997,Nicholson and Mather 1996). This definition assumes that all Borrelia genospecies are considered equally hazardous for humans. Our study of pathogenicity of the various Borrelia 


\section{Chapter 7}

genospecies and genotypes (Chapter 6) favours the separate hazard assignment for the Borrelia genospecies. The combination of hazard and exposure (prevalence in questing ticks) would then allow individual genospecies/genotypes risk assessment.

Numerous studies addressed the topic of hazard for B. burgdorferi s.l. infection and the way the vertebrate hosts' composition influences this (Brisson et al. 2011,LoGiudice et al. 2003, Ruyts et al. 2016,Talleklint and Jaenson 1996). One of the most prominent controversies on how the acarological risk varies according to the vertebrate community is that around the "dilution effect theory". Its initiators, studying habitats in North America, found that increased biodiversity will lead to an increased abundance of unsuitable transmission vertebrates for B. burgdorferi s.s., with the ensuing "dilution" (reduction) of the spirochetal infection in the questing ticks (LoGiudice et al. 2008, LoGiudice et al. 2003, Ostfeld and Keesing 2000). Conversely, other authors have suggested that, on the contrary, biodiversity will only amplify the risk, due to the abundance of hosts that will implicitly lead to an increased abundance of the ticks (Ogden and Tsao 2009,Randolph and Dobson 2012). One of the few vertebrate groups that have been identified as incompetent for Borrelia burgdorferi s.l. amplification or transmission is that of Artiodactyls (Jaenson and Talleklint 1992, Matuschka et al. 1993). The introduction of more such animals in a habitat will result, therefore, in a reduction of Borrelia burgdorferi s.l. infection in ticks. However, these animals feed a very large number of ticks, and especially adult ticks, which, in turn will result in a higher number of questing ticks as these animals act as reproductive hosts. Thus, even if the prevalence of $B$. burgdorferi s.l. infection in ticks is decreased, the overall density of Borrelia infected ticks might follow the opposite trend, while introducing other tick-borne pathogens in the system, such as Anaplasma phagocytophilum, Babesia divergens and Babesia venatorum.

From a meta-analysis study it resulted that the overall mean prevalence of B. burgdorferi s.l. in ticks in Europe is $13.7 \%$ (Rauter and Hartung 2005), with a lower average for nymphs (10.1\%) comparing to adults (18.6\%). In countrywide studies involving multiple areas in The Netherlands, comparable prevalences 11.8 to $15.6 \%$ - of $B$. burgdorferi s.I were found in questing ticks, with large and consistent variations between various locations (Coipan et al. 2013c,Takken et al. in press). We also found that in areas where tick densities were highest, the mean prevalence of Borrelia infection had lower values (Chapter 5). The hypothesis of a constant prevalence over the range of questing ticks density was tested and the results indicated a slightly negative correlation of the prevalence with the tick density. That implies that the density of ticks infected with $B$. burgdorferi s.l. decreases as the density of questing ticks increases. Plotting the density of infected questing ticks as an exponential function of the questing 
ticks' densities, however, revealed that over the usual range of questing ticks densities the density of infected ticks is also increasing, and the downward trend might be observed only for questing ticks densities of over 200/100 m2 (Chapter 5). This observation is consistent with the finding that, in Europe, the density of Borrelia infected ticks depends much more on the density of all ticks than on the infection prevalence, and that only in areas where the tick density is unusually high (100-450/100 m2) is the infection prevalence consistently low (Randolph 2001). This hypothesis is also confirmed by a recent study of Takken et al. (in press) and by the 10 years longitudinal study of density of ticks and their infection prevalence with tick-borne pathogens at Duin en Kruidberg (The Netherlands, Chapter 5); there, the density of infected nymphs followed the same trend as the overall density of questing nymphs, while the prevalence of infection with B. burgdorferi s.l. remained constant (Chapter 5). It is, thus, obvious that the density of questing nymphs is the main driver of the acarological risk of human exposure to $B$. burgdorferi s.l. What drives the variations in nymphal density might be mostly rodent abundance and climate and is surely an interesting topic of further research.

Furthermore, each of the 20 genospecies of $B$. burgdorferi s.l. has its own vertebrate host spectrum. For example, $B$. afzelii is mainly transmitted by small mammals, while $B$. garinii is mainly transmitted by birds (Hanincova et al. 2003a, Hanincova et al. 2003b). Under these circumstances, a decrease of biodiversity of one vertebrate class on the expense of the increase of another would lead to the "dilution" of one Borrelia burgdorferi s.l. genospecies but to the increase in prevalence of another. It is also generally accepted that with the increase in biodiversity there will also be an increase in the diversity of zoonotic agents (Guernier et al. 2004,Hechinger and Lafferty 2005). Surely, biodiversity might be affect de abundance of several of these pathogens, but the key question for public health therefore lies in the accumulation of all the hazards, weighed by their abundance and (potential) disease burden in humans.

Although our understanding of the ecology and pathogenicity of $B$. burgdorferi s.l. has come very far in the last decades, there are still knowledge gaps, especially regarding the recently described genospecies. Borrelia bavariensis, for that matter, is a genospecies that is known to be maintained in enzootic cycles by the same rodent and ticks species as $B$. afzelii. Why is it then that its prevalence in questing ticks is less than $1 \%$ ? One of the possible answers is the existence of alternative vertebrate hosts and vectors. Another one would be the increased virulence with subsequent rapid clearance by the immune response of the host, in a similar manner with Borrelia miyamotoi. Should that be the case, how do some of the bacteria escape the immune system and what determines their neurotropism, as observed in humans? All these questions will 


\section{Chapter 7}

most probably find the answer in future research. For now, the only certitude is that the acarological risk for this and possibly other B. burgdorferi s.l. genospecies, is not the best indicator for use in risk assessment.

While the genetic make-up of the Lyme borreliosis spirochaetes undoubtfully plays a role in the clinical manifestations observed in humans, it alone cannot fully explain the observed variation in prevalence and severity of the various clinical manifestations. The pathogenesis of chronic Lyme disease seems to be a combination of persistent infection and autoimmunity (Singh and Girschick 2004), as it was shown in the case of chronic joint inflammation (Steere et al. 2001) or Lyme carditis (Raveche et al. 2005). Early recognition/treatment of the disease can prevent irreversible damage done by the (immune reaction to the) infection. Furthermore, the genetic/immunological status of the infected person might be equally important (Bramwell et al. 2014,Schroder et al. 2005). The wide range in outcomes in untreated patients reflects most probably the interplay between spirochaetal virulence and host immune response.

\section{Concluding remarks}

In this thesis, I have shown that both ecological and clinical studies are necessary to be able to address the public health issue that is collectively called Lyme borreliosis. Although many vertebrate species have been shown to act as reservoirs for $B$. burgdorferi s.l. and hosts for I. ricinus, it is only few of them that account for maintenance of most ticks and B. burgdorferi s.l. in enzootic cycles. The density of the vertebrates and the feeding preferences of the ticks dictate the prevalence of the various Borrelia genospecies in the different developmental stages of questing ticks e.g. the high prevalence of $B$. afzelii in questing nymphs is caused by the high proportion of larvae that feed on small rodents. Despite its high incidence in ticks and erythema migrans, in terms of disease burden (as measured by disability-adjusted life year), B. afzelii is probably of least concern for public health. Other Borrelia spirochaetes that are very seldom found in questing $I$. ricinus ticks, such as $B$. bavariensis, seem to be responsible for more serious clinical symptoms in humans. This implies that the prevalence of $B$. burgdorferi s.l. in questing ticks does not necessarily reflect the incidence of human Lyme borreliosis. Thus, we cannot approximate the disease incidence, let alone disease burden, with the acarological risk or exposure. From that perspective, the aim of this thesis, to link the ecology of $B$. burgdorferi s.l. with its pathogenicity for humans, has not been fully achieved.

In my research, I have also shown that the host specificity of $B$. burgdorferi s.l. observed at genospecies level does not hold true for the intragenospecies level and that some genotypes have a higher propensity of causing disease than 
others. Furthermore, I have shown that there are other microorganisms that coinfect both the questing and the engorged larvae on the rodents implying similar transmission patterns. These factors, together with the variation in human immune response to the spirochaetes, may play an important role in the infectivity and pathogenesis of the spirochaetes. Future studies addressing the extent of the intraspecific genetic variation of $B$. burgdorferi s.l., the microbiome of the ticks and the variation in the human immune response might be able to fully link the ecology of $B$. burgdorferi s.l. with its pathogenicity for humans. 
Chapter 7 


\section{References}

Abascal F, Zardoya R and Telford MJ (2010) Translatorx: Multiple alignment of nucleotide sequences guided by amino acid translations. Nucleic acids research 38: W7-13.

Ackermann R, Kabatzki J, Boisten HP, Steere AC, Grodzicki RL, Hartung S and Runne U (1984) Ixodes ricinus spirochete and european erythema chronicum migrans disease. Yale J Biol Med 57: 573-580.

Alekseev AN, Dubinina HV, Van De Pol I and Schouls LM (2001) Identification of ehrlichia spp. And Borrelia burgdorferi in ixodes ticks in the baltic regions of russia. J Clin Microbiol 39: 2237-2242.

Altschul SF, Gish W, Miller W, Myers EW and Lipman DJ (1990) Basic local alignment search tool. J Mol Biol 215: 403-410.

Amore G, Tomassone L, Grego E, Ragagli C, Bertolotti L, Nebbia P, Rosati S and Mannelli A (2007) Borrelia lusitaniae in immature Ixodes ricinus (acari: Ixodidae) feeding on common wall lizards in tuscany, central italy. J Med Entomol 44: 303-307.

Amori G, Hutterer R, Kryštufek B, Yigit N, Mitsain G and Palomo LJ (2008) Apodemus flavicollis.

Available

at: http://dx.doi.org/10.2305/IUCN.UK.2008.RLTS.T1892A8699693.en. Accessed 15 Nov.

Anderson JF and Magnarelli LA (1993) Epizootiology of lyme disease-causing borreliae. Clin Dermatol 11: 339-351.

Andersson $M$ and Raberg $L$ (2011) Wild rodents and novel human pathogen candidatus Neoehrlichia mikurensis, southern sweden. Emerg Infect Dis 17: 1716-1718.

Andersson M, Scherman K and Raberg L (2014) Infection dynamics of the tick-borne pathogen "candidatus Neoehrlichia mikurensis" and coinfections with Borrelia afzelii in bank voles in southern sweden. Appl Environ Microbiol 80: 1645-1649.

Apollonio M, Andersen R and Putman R (2010) European ungulates and their management in the 21st century. Cambridge, UK ; New York, Cambridge University Press, xiii, 604 p. pp.

Avise JC (2007) On evolution, Johns Hopkins University Press.

Balmelli T and Piffaretti JC (1995) Association between different clinical manifestations of lyme disease and different species of Borrelia burgdorferi sensu lato. Res Microbiol 146: 329-340.

Baranton G, Postic D, Saint Girons I, Boerlin P, Piffaretti JC, Assous M and Grimont PA (1992) Delineation of Borrelia burgdorferi sensu stricto, Borrelia garinii sp. Nov., and group vs461 associated with lyme borreliosis. Int J Syst Bacteriol 42: 378.

Barbour A (2004) Specificity of borrelia-tick vector relationships. In: Gillespie SH and Smith GL eds., Microbe-vector interactions in vector-borne diseases, Cambridge, GB, Cambridge University Press, pp. 383.

Barbour AG (1988) Plasmid analysis of Borrelia burgdorferi, the lyme disease agent. J Clin Microbiol 26: 475-478. 


\section{References}

Barbour AG, Bunikis J, Fish D and Hanincová K (2015) Association between body size and reservoir competence of mammals bearing Borrelia burgdorferi at an endemic site in the northeastern united states. Parasites \& vectors 8: 1.

Barbour AG, Burgdorfer W, Hayes SF, Péter O and Aeschlimann A (1983) Isolation of a cultivable spirochete from\&lt;i\&gt;/xodes ricinus\&lt;/i\&gt; ticks of switzerland. Current Microbiology 8: 123-126.

Barthold SW (1999) Specificity of infection-induced immunity among Borrelia burgdorferi sensu lato species. Infect Immun 67: 36-42.

Bartoń K (2014) Mumin: Multi-model inference.

Belongia EA (2002) Epidemiology and impact of coinfections acquired from ixodes ticks. Vector borne and zoonotic diseases (Larchmont, NY) 2: 265-273.

Benjamini $Y$ and Hochberg $Y$ (1995) Controlling the false discovery rate: A practical and powerful approach to multiple testing. Journal of the Royal Statistical Society Series B (Methodological) 57: 289-300.

Bhide MR, Travnicek M, Levkutova M, Curlik J, Revajova V and Levkut M (2005) Sensitivity of borrelia genospecies to serum complement from different animals and human: A host-pathogen relationship. FEMS Immunol Med Microbiol 43: 165172.

Bingsohn L, Beckert A, Zehner R, Kuch U, Oehme R, Kraiczy P and Amendt J (2013) Prevalences of tick-borne encephalitis virus and Borrelia burgdorferi sensu lato in Ixodes ricinus populations of the rhine-main region, germany. Ticks and tick-borne diseases 4: 207-213.

Bissett ML and Hill W (1987) Characterization of Borrelia burgdorferi strains isolated from ixodes pacificus ticks in california. Journal of clinical microbiology 25: 22962301.

Bonnet S, Jouglin M, L'Hostis M and Chauvin A (2007) Babesia sp. Eu1 from roe deer and transmission within Ixodes ricinus. Emerg Infect Dis 13: 1208-1210.

Bown KJ, Begon M, Bennett M, Birtles RJ, Burthe S, Lambin X, Telfer S, Woldehiwet Z and Ogden NH (2006) Sympatric ixodes trianguliceps and Ixodes ricinus ticks feeding on field voles (microtus agrestis): Potential for increased risk of Anaplasma phagocytophilum in the united kingdom? Vector Borne Zoonotic Dis 6: 404-410.

Boyard C, Vourc'h G and Barnouin J (2008) The relationships between Ixodes ricinus and small mammal species at the woodland-pasture interface. Exp Appl Acarol 44: 6176.

Bramwell KK, Teuscher C and Weis JJ (2014) Forward genetic approaches for elucidation of novel regulators of lyme arthritis severity. Frontiers in cellular and infection microbiology 4: 76.

Brinck P, Johnels A, Lundholm B, Svedmyr A, von Zeipel G and Zetterberg B (1967) Small mammals in sweden as hosts of tick-borne encephalitis virus and vagrant ectoparasites. Oikos 18: 124-134. 
Brisson D, Brinkley C, Humphrey PT, Kemps BD and Ostfeld RS (2011) It takes a community to raise the prevalence of a zoonotic pathogen. Interdisciplinary perspectives on infectious diseases 2011: 741406.

Brisson D, Drecktrah D, Eggers CH and Samuels DS (2012) Genetics of Borrelia burgdorferi. Annual review of genetics 46: 515-536.

Brisson D and Dykhuizen DE (2004) Ospc diversity in Borrelia burgdorferi: Different hosts are different niches. Genetics 168: 713-722.

Brunner JL and Ostfeld RS (2008) Multiple causes of variable tick burdens on smallmammal hosts. Ecology 89: 2259-2272.

Bunikis J, Garpmo U, Tsao J, Berglund J, Fish D and Barbour AG (2004) Sequence typing reveals extensive strain diversity of the lyme borreliosis agents Borrelia burgdorferi in north america and Borrelia afzelii in europe. Microbiology 150: 1741-1755.

Burgdorfer W, Barbour AG, Hayes SF, Benach JL, Grunwaldt E and Davis JP (1982) Lyme disease-a tick-borne spirochetosis? Science 216: 1317-1319.

Burgdorfer W, Barbour AG, Hayes SF, Peter O and Aeschlimann A (1983) Erythema chronicum migrans--a tickborne spirochetosis. Acta tropica 40: 79-83.

Burnham KP and Anderson DR (2004) Multimodel inference: Understanding aic and bic in model selection. Sociological Methods \& Research 33: 261-304.

Burri C, Dupasquier C, Bastic V and Gern L (2011) Pathogens of emerging tick-borne diseases, Anaplasma phagocytophilum, rickettsia spp., and babesia spp., in ixodes ticks collected from rodents at four sites in switzerland (canton of bern). Vector borne and zoonotic diseases (Larchmont, NY) 11: 939-944.

Burri C, Schumann O, Schumann C and Gern L (2014) Are apodemus spp. Mice and Myodes glareolus reservoirs for Borrelia miyamotoi, candidatus Neoehrlichia mikurensis, rickettsia helvetica, r. Monacensis and Anaplasma phagocytophilum? Ticks Tick Borne Dis.

Carbone C, Cowlishaw G, Isaac NJ and Rowcliffe JM (2005) How far do animals go? Determinants of day range in mammals. The American Naturalist 165: 290-297.

Casjens SR, Mongodin EF, Qiu WG, Dunn JJ, Luft BJ, Fraser-Liggett CM and Schutzer SE (2011) Whole-genome sequences of two Borrelia afzelii and two Borrelia garinii lyme disease agent isolates. J Bacteriol 193: 6995-6996.

Casjens SR, Mongodin EF, Qiu WG, Luft BJ, Schutzer SE, Gilcrease EB, Huang WM, Vujadinovic M, Aron JK, Vargas LC, Freeman S, Radune D, Weidman JF, Dimitrov GI, Khouri HM, Sosa JE, Halpin RA, Dunn JJ and Fraser CM (2012) Genome stability of lyme disease spirochetes: Comparative genomics of Borrelia burgdorferi plasmids. PLoS One 7: e33280.

Cassatt DR, Patel NK, Ulbrandt ND and Hanson MS (1998) Dbpa, but not ospa, is expressed by Borrelia burgdorferi during spirochetemia and is a target for protective antibodies. Infect Immun 66: 5379-5387. 
Chao A, Chazdon RL, Colwell RK and Shen T-J (2005) A new statistical approach for assessing similarity of species composition with incidence and abundance data. Ecology Letters 8: 148-159.

Christova I and Gladnishka T (2005) Prevalence of infection with francisella tularensis, Borrelia burgdorferi sensu lato and Anaplasma phagocytophilum in rodents from an endemic focus of tularemia in bulgaria. Ann Agric Environ Med 12: 149-152.

Christova I, Van De Pol J, Yazar S, Velo E and Schouls L (2003) Identification of Borrelia burgdorferi sensu lato, anaplasma and ehrlichia species, and spotted fever group rickettsiae in ticks from southeastern europe. Eur J Clin Microbiol Infect Dis 22: 535-542.

Chu CY, Liu W, Jiang BG, Wang DM, Jiang WJ, Zhao QM, Zhang PH, Wang ZX, Tang GP, Yang $\mathrm{H}$ and Cao WC (2008) Novel genospecies of Borrelia burgdorferi sensu lato from rodents and ticks in southwestern china. J Clin Microbiol 46: 3130-3133.

Coipan EC, Fonville M, Tijsse-Klasen E, van der Giessen JW, Takken W, Sprong H and Takumi K (2013a) Geodemographic analysis of Borrelia burgdorferi sensu lato using the 5s-23s rdna spacer region. Infect Genet Evol 17C: 216-222.

Coipan EC, Fonville M, Tijsse-Klasen E, van der Giessen JW, Takken W, Sprong H and Takumi K (2013b) Geodemographic analysis of Borrelia burgdorferi sensu lato using the $5 \mathrm{~s}-23 \mathrm{~s}$ rdna spacer region. Infection, genetics and evolution : journal of molecular epidemiology and evolutionary genetics in infectious diseases 17: 216222.

Coipan EC, Jahfari S, Fonville M, Maassen CB, van der Giessen J, Takken W, Takumi K and Sprong $\mathrm{H}$ (2013c) Spatiotemporal dynamics of emerging pathogens in questing Ixodes ricinus. Frontiers in cellular and infection microbiology 3: 36.

Coipan EC, Jahfari S, Fonville M, Oei GA, Spanjaard L, Takumi K, Hovius JW and Sprong H (2016) Imbalanced presence of Borrelia burgdorferi s.L. Multilocus sequence types in clinical manifestations of lyme borreliosis. Infect Genet Evol 42: 66-76.

Collares-Pereira M, Couceiro S, Franca I, Kurtenbach K, Schafer SM, Vitorino L, Goncalves L, Baptista S, Vieira ML and Cunha C (2004) First isolation of Borrelia lusitaniae from a human patient. Journal of clinical microbiology 42: 1316-1318.

Colwell RK (2013) Estimates: Statistical estimation of species richness and shared species from samples.

Colwell RK and Coddington JA (1994) Estimating terrestrial biodiversity through extrapolation. Philosophical Transactions of the Royal Society of London B: Biological Sciences 345: 101-118.

Colwell RK, Mao CX and Chang J (2004) Interpolating, extrapolating, and comparing incidence-based species accumulation curves. Ecology 85: 2717-2727.

Cook RD (1977) Detection of influential observation in linear regression. Technometrics 19: 15-18.

Cosson JF, Michelet L, Chotte J, Le Naour E, Cote M, Devillers E, Poulle ML, Huet D, Galan M, Geller J, Moutailler S and Vayssier-Taussat M (2014) Genetic characterization 
of the human relapsing fever spirochete Borrelia miyamotoi in vectors and animal reservoirs of lyme disease spirochetes in france. Parasit Vectors 7: 233.

Cramp S and Perrins CM (1994) Handbook of the birds of europe, the middle east and north africa: The birds of the western palearctic. Oxford, Oxford University Press.

Dantas-Torres F, Chomel BB and Otranto D (2012) Ticks and tick-borne diseases: A one health perspective. Trends Parasitol 28: 437-446.

De Boer R, Hovius KE, Nohlmans MK and Gray JS (1993) The woodmouse (Apodemus sylvaticus) as a reservoir of tick-transmitted spirochetes (Borrelia burgdorferi) in the netherlands. Zentralbl Bakteriol 279: 404-416.

de Carvalho IL, Fonseca JE, Marques JG, Ullmann A, Hojgaard A, Zeidner N and Nuncio MS (2008) Vasculitis-like syndrome associated with Borrelia lusitaniae infection. Clin Rheumatol 27: 1587-1591.

de Carvalho IL, Zeidner N, Ullmann A, Hojgaard A, Amaro F, Ze-Ze L, Alves MJ, de Sousa R, Piesman J and Nuncio MS (2010) Molecular characterization of a new isolate of Borrelia lusitaniae derived from Apodemus sylvaticus in portugal. Vector Borne Zoonotic Dis 10: 531-534.

De La Fuente J, Naranjo V, Ruiz-Fons F, Hofle U, Fernandez De Mera IG, Villanua D, Almazan C, Torina A, Caracappa S, Kocan KM and Gortazar C (2005) Potential vertebrate reservoir hosts and invertebrate vectors of anaplasma marginale and A. phagocytophilum in central spain. Vector Borne Zoonotic Dis 5: 390-401.

Derdakova M, Beati L, Pet'ko B, Stanko M and Fish D (2003) Genetic variability within Borrelia burgdorferi sensu lato genospecies established by pcr-single-strand conformation polymorphism analysis of the rrfa-rrlb intergenic spacer in Ixodes ricinus ticks from the czech republic. Appl Environ Microbiol 69: 509-516.

Derdakova $M$ and Lencakova D (2005) Association of genetic variability within the Borrelia burgdorferi sensu lato with the ecology, epidemiology of lyme borreliosis in europe. Ann Agric Environ Med 12: 165-172.

Devevey G, Dang T, Graves CJ, Murray S and Brisson D (2015) First arrived takes all: Inhibitory priority effects dominate competition between co-infecting Borrelia burgdorferi strains. BMC Microbiol 15: 61.

Dister SW, Fish D, Bros SM, Frank DH and Wood BL (1997) Landscape characterization of peridomestic risk for lyme disease using satellite imagery. The American journal of tropical medicine and hygiene 57: 687-692.

Diza E, Papa A, Vezyri E, Tsounis S, Milonas I and Antoniadis A (2004) Borrelia valaisiana in cerebrospinal fluid. Emerging infectious diseases 10: 1692-1693.

Dubska L, Literak I, Kocianova E, Taragelova V, Sverakova V, Sychra O and Hromadko M (2011) Synanthropic birds influence the distribution of borrelia species: Analysis of Ixodes ricinus ticks feeding on passerine birds. Appl Environ Microbiol 77: 11151117.

Dubska L, Literak I, Kocianova E, Taragelova V and Sychra O (2009) Differential role of passerine birds in distribution of borrelia spirochetes, based on data from ticks 
collected from birds during the postbreeding migration period in central europe. Appl Environ Microbiol 75: 596-602.

Dubska L, Literak I, Kverek P, Roubalova E, Kocianova E and Taragelova V (2012) Tickborne zoonotic pathogens in ticks feeding on the common nightingale including a novel strain of rickettsia sp. Ticks Tick Borne Dis 3: 265-268.

Duh D, Petrovec M and Avsic-Zupanc T (2005) Molecular characterization of human pathogen babesia eu1 in Ixodes ricinus ticks from slovenia. J Parasitol 91: 463-465.

Durand J, Jacquet M, Paillard L, Rais O, Gern L and Voordouw MJ (2015) Cross-immunity and community structure of a multiple-strain pathogen in the tick vector. Appl Environ Microbiol 81: 7740-7752.

Durrett R (2008) Probability models for DNA sequence evolution, Springer.

Dykhuizen DE, Brisson D, Sandigursky S, Wormser GP, Nowakowski J, Nadelman RB and Schwartz I (2008) The propensity of different Borrelia burgdorferi sensu stricto genotypes to cause disseminated infections in humans. The American journal of tropical medicine and hygiene 78: 806-810.

ECDC (2011) Meeting report. Second expert consultation on tick-borne diseases with emphasis on lyme borreliosis and tick-borne encephalitis. Available at: http://ecdc.europa.eu/en/publications/Publications/Tick-borne-diseasesmeeting-report.pdf.

Edgar RC (2004) Muscle: Multiple sequence alignment with high accuracy and high throughput. Nucleic acids research 32: 1792-1797.

Eiffert H, Ohlenbusch A, Christen HJ, Thomssen R, Spielman A and Matuschka FR (1995) Nondifferentiation between lyme disease spirochetes from vector ticks and human cerebrospinal fluid. J Infect Dis 171: 476-479.

Estrada-Pena A, Osacar JJ, Pichon B and Gray JS (2005) Hosts and pathogen detection for immature stages of Ixodes ricinus (acari: Ixodidae) in north-central spain. Exp Appl Acarol 37: 257-268.

Ewens WJ (1972) The sampling theory of selectively neutral alleles. Theor Popul Biol 3: 87-112.

Excoffier L and Lischer HE (2010) Arlequin suite ver 3.5: A new series of programs to perform population genetics analyses under linux and windows. Mol Ecol Resour 10: 564-567.

Fay JC and $\mathrm{Wu} \mathrm{Cl}$ (2000) Hitchhiking under positive darwinian selection. Genetics 155: 1405-1413.

Fehr JS, Bloemberg GV, Ritter C, Hombach M, Luscher TF, Weber R and Keller PM (2010) Septicemia caused by tick-borne bacterial pathogen candidatus Neoehrlichia mikurensis. Emerg Infect Dis 16: 1127-1129.

Ferreri L, Giacobini M, Bajardi P, Bertolotti L, Bolzoni L, Tagliapietra V, Rizzoli A and Rosa $R$ (2014) Pattern of tick aggregation on mice: Larger than expected distribution 
tail enhances the spread of tick-borne pathogens. PLoS Comput Biol 10: e1003931.

Fietz J, Tomiuk J, Matuschka FR and Richter D (2014) Seasonal prevalence of lyme disease spirochetes in a heterothermic mammal, the edible dormouse (glis glis). Appl Environ Microbiol 80: 3615-3621.

Fingerle V, Schulte-Spechtel UC, Ruzic-Sabljic E, Leonhard S, Hofmann H, Weber K, Pfister $\mathrm{K}$, Strle F and Wilske B (2008) Epidemiological aspects and molecular characterization of Borrelia burgdorferi s.L. From southern germany with special respect to the new species Borrelia spielmanii sp. Nov. Int J Med Microbiol 298: 279-290.

Fonville M, Friesema IH, Hengeveld PD, Docters van Leeuwen A, Jahfari S, Harms MG, van Vliet AJ, Hofhuis A, van Pelt W, Sprong $H$ and van den Wijngaard CC (2014) Human exposure to tickborne relapsing fever spirochete Borrelia miyamotoi, the netherlands. Emerg Infect Dis 20: 1244-1245.

Fraenkel CJ, Garpmo U and Berglund J (2002) Determination of novel borrelia genospecies in swedish Ixodes ricinus ticks. J Clin Microbiol 40: 3308-3312.

Franke J, Fritzsch J, Tomaso H, Straube E, Dorn W and Hildebrandt A (2010) Coexistence of pathogens in host-seeking and feeding ticks within a single natural habitat in central germany. Appl Environ Microbiol 76: 6829-6836.

Franke J, Hildebrandt A and Dorn W (2013) Exploring gaps in our knowledge on lyme borreliosis spirochaetes--updates on complex heterogeneity, ecology, and pathogenicity. Ticks Tick Borne Dis 4: 11-25.

Fraser CM, Casjens S, Huang WM, Sutton GG, Clayton R, Lathigra R, White O, Ketchum KA, Dodson R, Hickey EK, Gwinn M, Dougherty B, Tomb JF, Fleischmann RD, Richardson D, Peterson J, Kerlavage AR, Quackenbush J, Salzberg S, Hanson M, van Vugt R, Palmer N, Adams MD, Gocayne J, Weidman J, Utterback T, Watthey L, McDonald L, Artiach P, Bowman C, Garland S, Fuji C, Cotton MD, Horst K, Roberts K, Hatch B, Smith HO and Venter JC (1997) Genomic sequence of a lyme disease spirochaete, Borrelia burgdorferi. Nature 390: 580-586.

Fu YX (1997) Statistical tests of neutrality of mutations against population growth, hitchhiking and background selection. Genetics 147: 915-925.

Fukunaga M, Hamase A, Okada K, Inoue H, Tsuruta Y, Miyamoto K and Nakao M (1996) Characterization of spirochetes isolated from ticks (ixodes tanuki, ixodes turdus, and ixodes columnae) and comparison of the sequences with those of Borrelia burgdorferi sensu lato strains. Appl Environ Microbiol 62: 2338-2344.

Gassner F, Takken W, Plas CL, Kastelein P, Hoetmer AJ, Holdinga M and van Overbeek LS (2013) Rodent species as natural reservoirs of Borrelia burgdorferi sensu lato in different habitats of Ixodes ricinus in the netherlands. Ticks Tick Borne Dis 4: 452458.

Gassner F, van Vliet AJ, Burgers SL, Jacobs F, Verbaarschot P, Hovius EK, Mulder S, Verhulst NO, van Overbeek LS and Takken W (2011) Geographic and temporal 
variations in population dynamics of Ixodes ricinus and associated borrelia infections in the netherlands. Vector Borne Zoonotic Dis 11: 523-532.

Gatzmann F, Metzler D, Krebs S, Blum H, Sing A, Takano A, Kawabata H, Fingerle V, Margos $G$ and Becker NS (2015) Ngs population genetics analyses reveal divergent evolution of a lyme borreliosis agent in europe and asia. Ticks Tick Borne Dis 6: 344-351.

Gazumyan A, Schwartz JJ, Liveris D and Schwartz I (1994) Sequence analysis of the ribosomal rna operon of the lyme disease spirochete, Borrelia burgdorferi. Gene 146: 57-65.

Gern L, Douet V, Lopez Z, Rais O and Cadenas FM (2010) Diversity of borrelia genospecies in Ixodes ricinus ticks in a lyme borreliosis endemic area in switzerland identified by using new probes for reverse line blotting. Ticks Tick Borne Dis 1: 23-29.

Gern L and Humair PF (2002a) Ecology of Borrelia burgdorferi sensu lato in europe. In: Gray JS, Kahl O, Lane RS and Stanek G eds., Lyme borreliosis: Biology, epidemiology and control, Wallingford, Oxon, UK, CAB International, pp. 149-174.

Gern L and Humair PF (2002b) Ecology of Borrelia burgdorferi sensu lato in europe. In: J. Gray OK, R.s.Lane and G. Stanek,ed. Lyme borreliosis: Biology, epidemiology and control, CABI International.

Gern L and Rais O (1996) Efficient transmission of Borrelia burgdorferi between cofeeding Ixodes ricinus ticks (acari: Ixodidae). J Med Entomol 33: 189-192.

Gern L, Rouvinez E, Toutoungi LN and Godfroid E (1997) Transmission cycles of Borrelia burgdorferi sensu lato involving Ixodes ricinus and/or i. Hexagonus ticks and the european hedgehog, erinaceus europaeus, in suburban and urban areas in switzerland. Folia Parasitol (Praha) 44: 309-314.

Ginsberg HS (2008) Potential effects of mixed infections in ticks on transmission dynamics of pathogens: Comparative analysis of published records. Experimental \& applied acarology 46: 29-41.

Glass GE, Amerasinghe FP, Morgan JM, 3rd and Scott TW (1994) Predicting ixodes scapularis abundance on white-tailed deer using geographic information systems. The American journal of tropical medicine and hygiene 51: 538-544.

Glass GE, Schwartz BS, Morgan JM, 3rd, Johnson DT, Noy PM and Israel E (1995) Environmental risk factors for lyme disease identified with geographic information systems. American journal of public health 85: 944-948.

Glatz M, Muellegger RR, Hizo-Teufel C and Fingerle V (2014) Low prevalence of Borrelia bavariensis in Ixodes ricinus ticks in southeastern austria. Ticks and tick-borne diseases 5: 649-650.

Gliddon C and Strobeck C (1975) Necessary and sufficient conditions for multiple-niche polymorphism in haploids. The American Naturalist 109: 233-235.

Grankvist A, Moore ER, Svensson Stadler L, Pekova S, Bogdan C, Geissdorfer W, GripLinden J, Brandstrom K, Marsal J, Andreasson K, Lewerin C, Welinder-Olsson C and 
Wenneras C (2015) Multilocus sequence analysis of clinical "candidatus Neoehrlichia mikurensis" strains from europe. J Clin Microbiol 53: 3126-3132.

Gray J (1991) The development and seasonal activity of the tick Ixodes ricinus: A vector of lyme borreliosis. Review of Medical and Veterinary Entomology 79: 323-333.

Gray J, von Stedingk LV, Gurtelschmid M and Granstrom M (2002) Transmission studies of babesia microti in Ixodes ricinus ticks and gerbils. J Clin Microbiol 40: 12591263.

Gray JS (1998) Review the ecology of ticks transmitting lyme borreliosis. Experimental \& Applied Acarology 22: 249-258.

Gray JS, Kahl O, Janetzki C and Stein J (1992) Studies on the ecology of lyme disease in a deer forest in county galway, ireland. J Med Entomol 29: 915-920.

Gray JS, Kahl O, Janetzki C, Stein J and Guy E (1995) The spatial distribution of Borrelia burgdorferi-infected Ixodes ricinus in the connemara region of county galway, ireland. Exp Appl Acarol 19: 163-172.

Gray JS, Kahl O, Robertson JN, Daniel M, Estrada-Pena A, Gettinby G, Jaenson TG, Jensen $\mathrm{P}$, Jongejan $\mathrm{F}$, Korenberg $\mathrm{E}$, Kurtenbach $\mathrm{K}$ and Zeman $\mathrm{P}$ (1998) Lyme borreliosis habitat assessment. Zentralbl Bakteriol 287: 211-228.

Gray JS, Kirstein F, Robertson JN, Stein J and Kahl O (1999) Borrelia burgdorferi sensu lato in Ixodes ricinus ticks and rodents in a recreational park in south-western ireland. Exp Appl Acarol 23: 717-729.

Gray JS, Robertson JN and Key S (2000) Limited role of rodents as reservoirs of Borrelia burgdorferi sensu lato in ireland. Eur J Epidemiol 16: 101-103.

Gregory RD, Vorisek P, Van Strien A, Gmelig Meyling AW, Jiguet F, Fornasari L, Reif J, Chylarecki P and Burfield IJ (2007) Population trends of widespread woodland birds in europe. Ibis 149: 78-97.

Gromko MH (1977) What is frequency-dependent selection? Evolution 31: 438-442.

Guernier V, Hochberg ME and Guegan JF (2004) Ecology drives the worldwide distribution of human diseases. PLoS Biol 2: e141.

Guindon S, Dufayard JF, Lefort V, Anisimova M, Hordijk W and Gascuel O (2010) New algorithms and methods to estimate maximum-likelihood phylogenies: Assessing the performance of phyml 3.0. Systematic biology 59: 307-321.

Hanincova K, Mukherjee P, Ogden NH, Margos G, Wormser GP, Reed KD, Meece JK, Vandermause MF and Schwartz I (2013) Multilocus sequence typing of Borrelia burgdorferi suggests existence of lineages with differential pathogenic properties in humans. PloS one 8: e73066.

Hanincova K, Schafer SM, Etti S, Sewell HS, Taragelova V, Ziak D, Labuda M and Kurtenbach K (2003a) Association of Borrelia afzelii with rodents in europe. Parasitology 126: 11-20. 


\section{References}

Hanincova K, Taragelova V, Koci J, Schafer SM, Hails R, Ullmann AJ, Piesman J, Labuda M and Kurtenbach K (2003b) Association of Borrelia garinii and B. valaisiana with songbirds in slovakia. Appl Environ Microbiol 69: 2825-2830.

Harrison A, Bown KJ, Montgomery WI and Birtles RJ (2012) Ixodes ricinus is not an epidemiologically relevant vector of bartonella species in the wood mouse (Apodemus sylvaticus). Vector Borne Zoonotic Dis 12: 366-371.

Hartelt K, Oehme R, Frank H, Brockmann SO, Hassler D and Kimmig P (2004) Pathogens and symbionts in ticks: Prevalence of Anaplasma phagocytophilum (ehrlichia sp.), wolbachia sp., rickettsia sp., and babesia sp. In southern germany. Int J Med Microbiol 293 Suppl 37: 86-92.

Hasle G, Bjune GA, Midthjell L, Roed KH and Leinaas HP (2011) Transport of Ixodes ricinus infected with borrelia species to norway by northward-migrating passerine birds. Ticks Tick Borne Dis 2: 37-43.

Haven J, Vargas LC, Mongodin EF, Xue V, Hernandez Y, Pagan P, Fraser-Liggett CM, Schutzer SE, Luft BJ, Casjens SR and Qiu WG (2011) Pervasive recombination and sympatric genome diversification driven by frequency-dependent selection in Borrelia burgdorferi, the lyme disease bacterium. Genetics 189: 951-966.

Hechinger RF and Lafferty KD (2005) Host diversity begets parasite diversity: Bird final hosts and trematodes in snail intermediate hosts. Proc Biol Sci 272: 1059-1066.

Heidrich J (2000) Untersuchungen zur prävalenz von Borrelia burgdorferi sensu lato beim rotfuchs (vulpes vulpes) in ostbrandenburg, Freien Universität Berlin, Berlin.

Heidrich J, Schonberg A, Steuber S, Nockler K, Schulze P, Voigt WP and Schein E (1999) Investigation of skin samples from red foxes (vulpes vulpes) in eastern brandenburg (germany) for the detection of Borrelia burgdorferi s. L. Zentralbl Bakteriol 289: 666-672.

Hellgren O, Andersson M and Raberg L (2011) The genetic structure of Borrelia afzelii varies with geographic but not ecological sampling scale. J Evol Biol 24: 159-167.

Heylen D, Fonville $M$, van Leeuwen AD and Sprong $H$ (2016) Co-infections and transmission dynamics in a tick-borne bacterium community exposed to songbirds. Environ Microbiol 18: 988-996.

Heylen D, Tijsse E, Fonville M, Matthysen E and Sprong H (2013) Transmission dynamics of Borrelia burgdorferi s.L. In a bird tick community. Environ Microbiol 15: 663673.

Heyman P, Cochez C, Hofhuis A, van der Giessen J, Sprong H, Porter SR, Losson B, Saegerman C, Donoso-Mantke O, Niedrig M and Papa A (2010) A clear and present danger: Tick-borne diseases in europe. Expert Rev Anti Infect Ther 8: 33 50 .

Hildebrandt A, Pauliks K, Sachse S and Straube E (2010) Coexistence of borrelia spp. And babesia spp. In Ixodes ricinus ticks in middle germany. Vector Borne Zoonotic Dis 10: 831-837. 
Hiraoka H, Shimada Y, Sakata Y, Watanabe M, Itamoto K, Okuda M, Masuzawa T and Inokuma H (2007) Detection of Borrelia garinii, borrelia tanukii and borrelia sp. Closely related to Borrelia valaisiana in ixodes ticks removed from dogs and cats in japan. Vet Parasitol 144: 188-192.

Hoen AG, Margos G, Bent SJ, Diuk-Wasser MA, Barbour A, Kurtenbach K and Fish D (2009) Phylogeography of Borrelia burgdorferi in the eastern united states reflects multiple independent lyme disease emergence events. Proceedings of the National Academy of Sciences of the United States of America 106: 15013-15018.

Hofhuis A, van der Giessen JW, Borgsteede FH, Wielinga PR, Notermans DW and van Pelt W (2006) Lyme borreliosis in the netherlands: Strong increase in gp consultations and hospital admissions in past 10 years. Euro Surveill 11: E060622 060622.

Hofmeester TR, Coipan EC, van Wieren SE, Prins HHT, Takken W and Sprong H (2016) Few vertebrate species dominate the Borrelia burgdorferi s.L. Life cycle. Environmental Research Letters 11: 43001-43016.

Holden K, Hodzic E, Feng S, Freet KJ, Lefebvre RB and Barthold SW (2005) Coinfection with Anaplasma phagocytophilum alters Borrelia burgdorferi population distribution in c3h/hen mice. Infect Immun 73: 3440-3444.

Hovius JW, de Wever B, Sohne M, Brouwer MC, Coumou J, Wagemakers A, Oei A, Knol H, Narasimhan S, Hodiamont CJ, Jahfari S, Pals ST, Horlings HM, Fikrig E, Sprong H and van Oers $\mathrm{MH}$ (2013) A case of meningoencephalitis by the relapsing fever spirochaete Borrelia miyamotoi in europe. Lancet 382: 658.

Hovius JW, Schuijt TJ, de Groot KA, Roelofs JJ, Oei GA, Marquart JA, de Beer R, van 't Veer C, van der Poll T, Ramamoorthi N, Fikrig E and van Dam AP (2008) Preferential protection of Borrelia burgdorferi sensu stricto by a salp15 homologue in Ixodes ricinus saliva. J Infect Dis 198: 1189-1197.

Hu CM, Humair PF, Wallich R and Gern L (1997) Apodemus sp. Rodents, reservoir hosts for Borrelia afzelii in an endemic area in switzerland. Zentralblatt fur Bakteriologie : international journal of medical microbiology 285: 558-564.

Huang ZY, de Boer WF, van Langevelde F, Olson V, Blackburn TM and Prins HH (2013) Species' life-history traits explain interspecific variation in reservoir competence: A possible mechanism underlying the dilution effect. PLoS One 8: e54341.

Hubalek Z (2009) Epidemiology of lyme borreliosis. Curr Probl Dermatol 37: 31-50.

Huegli D, Hu CM, Humair PF, Wilske B and Gern L (2002) Apodemus species mice are reservoir hosts of Borrelia garinii ospa serotype 4 in switzerland. Journal of clinical microbiology 40: 4735-4737.

Hugli D, Moret J, Rais O, Moosmann Y, Erard P, Malinverni R and Gern L (2009) Tick bites in a lyme borreliosis highly endemic area in switzerland. Int J Med Microbiol 299: 155-160.

Hulbert AJ (2008) Explaining longevity of different animals: Is membrane fatty acid composition the missing link? Age (Dordr) 30: 89-97. 


\section{References}

Hulinska D, Votypka J, Kriz B, Holinkova N, Novakova J and Hulinsky V (2007) Phenotypic and genotypic analysis of borrelia spp. Isolated from Ixodes ricinus ticks by using electrophoretic chips and real-time polymerase chain reaction. Folia microbiologica 52: 315-324.

Hulinska D, Votypka J, Plch J, Vlcek E, Valesova M, Bojar M, Hulinsky V and Smetana K (2002) Molecular and microscopical evidence of ehrlichia spp. And Borrelia burgdorferi sensu lato in patients, animals and ticks in the czech republic. New Microbiol 25: 437-448.

Humair P and Gern L (2000) The wild hidden face of lyme borreliosis in europe. Microbes Infect 2: 915-922.

Humair PF, Douet V, Moran Cadenas F, Schouls LM, Van De Pol I and Gern L (2007) Molecular identification of bloodmeal source in Ixodes ricinus ticks using $12 \mathrm{~s}$ rdna as a genetic marker. J Med Entomol 44: 869-880.

Humair PF and Gern L (1998) Relationship between Borrelia burgdorferi sensu lato species, red squirrels (sciurus vulgaris) and Ixodes ricinus in enzootic areas in switzerland. Acta Trop 69: 213-227.

Humair PF, Peter O, Wallich R and Gern L (1995) Strain variation of lyme disease spirochetes isolated from Ixodes ricinus ticks and rodents collected in two endemic areas in switzerland. J Med Entomol 32: 433-438.

Humair PF, Rais O and Gern L (1999) Transmission of Borrelia afzelii from apodemus mice and clethrionomys voles to Ixodes ricinus ticks: Differential transmission pattern and overwintering maintenance. Parasitology 118 ( Pt 1): 33-42.

Humair PF, Turrian N, Aeschilimann A and Gern L (1993a) Borrelia burgdorferi in a focus of lyme borreliosis: Epizootiologic contribution of small mammals. Folia Parasitol (Praha) 40: 65-70.

Humair PF, Turrian N, Aeschlimann A and Gern L (1993b) Ixodes ricinus immatures on birds in a focus of lyme borreliosis. Folia Parasitol (Praha) 40: 237-242.

Ismail N, Bloch KC and McBride JW (2010) Human ehrlichiosis and anaplasmosis. Clin Lab Med 30: 261-292.

Jacquot M, Bisseux M, Abrial D, Marsot M, Ferquel E, Chapuis JL, Vourc'h G and Bailly X (2014) High-throughput sequence typing reveals genetic differentiation and host specialization among populations of the Borrelia burgdorferi species complex that infect rodents. PLoS One 9: e88581.

Jaenson TG and Talleklint L (1992) Incompetence of roe deer as reservoirs of the lyme borreliosis spirochete. J Med Entomol 29: 813-817.

Jaenson TG, Talleklint L, Lundqvist L, Olsen B, Chirico J and Mejlon H (1994) Geographical distribution, host associations, and vector roles of ticks (acari: Ixodidae, argasidae) in sweden. J Med Entomol 31: 240.

Jahfari S, Coipan EC, Fonville $M$, van Leeuwen AD, Hengeveld $P$, Heylen $D$, Heyman $P$, van Maanen C, Butler CM, Foldvari G, Szekeres S, van Duijvendijk G, Tack W, Rijks JM, van der Giessen J, Takken W, van Wieren SE, Takumi K and Sprong H (2014) 
Circulation of four Anaplasma phagocytophilum ecotypes in europe. Parasit Vectors 7: 365.

Jahfari S, Fonville M, Hengeveld P, Reusken C, Scholte EJ, Takken W, Heyman P, Medlock J, Heylen D, Kleve J and Sprong H (2012) Prevalence of Neoehrlichia mikurensis in ticks and rodents from north-west europe. Parasit Vectors 5: 74.

James MC, Furness RW, Bowman AS, Forbes KJ and Gilbert L (2011) The importance of passerine birds as tick hosts and in the transmission of Borrelia burgdorferi, the agent of lyme disease: A case study from scotland. Ibis 153: 293-302.

Jones KE, Patel NG, Levy MA, Storeygard A, Balk D, Gittleman JL and Daszak P (2008) Global trends in emerging infectious diseases. Nature 451: 990-993.

Jongejan F and Uilenberg $G$ (2004) The global importance of ticks. Parasitology 129: S3.

Jungnick S, Margos G, Rieger M, Dzaferovic E, Bent SJ, Overzier E, Silaghi C, Walder G, Wex F, Koloczek J, Sing A and Fingerle V (2015) Borrelia burgdorferi sensu stricto and Borrelia afzelii: Population structure and differential pathogenicity. International journal of medical microbiology : IJMM 305: 673-681.

Kahl O, Gern L, Eisen L and Lane RS (2002) Ecological research on Borrelia burgdorferi sensu lato: Terminology and some methodological pitfalls. In: Gray J, Kahl O, Lane $\mathrm{R}$ and Stanek $\mathrm{G}$ eds., Lyme borreliosis: Biology, epidemiology, and control, CABI.

Kaiser A, Seitz A and Strub O (2002) Prevalence of Borrelia burgdorferi sensu lato in the nightingale (luscinia megarhynchos) and other passerine birds. Int J Med Microbiol 291 Suppl 33: 75-79.

Karan LS, Koliasnikova NM, Toporkova MG, Makhneva MA, Nadezhdina MV, Esaulkova A, Romanenko VV, Arumova EA, Platonov AE and Maleev VV (2010) [usage of real time polymerase chain reaction for diagnostics of different tick-borne infections]. Zh Mikrobiol Epidemiol Immunobiol: 72-77.

Katoh K and Standley DM (2013) Mafft multiple sequence alignment software version 7: Improvements in performance and usability. Mol Biol Evol 30: 772-780.

Katoh $\mathrm{K}$ and Toh $\mathrm{H}$ (2008) Recent developments in the mafft multiple sequence alignment program. Brief Bioinform 9: 286-298.

Katoh K and Toh H (2010) Parallelization of the mafft multiple sequence alignment program. Bioinformatics 26: 1899-1900.

Kawabata H, Takano A, Kadosaka T, Fujita H, Nitta Y, Gokuden M, Honda T, Tomida J, Kawamura Y, Masuzawa T, Ishiguro F, Takada N, Yano Y, Andoh M, Ando S, Sato K, Takahashi $\mathrm{H}$ and Ohnishi M (2013) Multilocus sequence typing and DNA similarity analysis implicates that a Borrelia valaisiana-related sp. Isolated in japan is distinguishable from european B. valaisiana. J Vet Med Sci 75: 1201-1207.

Kiffner C, Vor T, Hagedorn P, Niedrig M and Ruhe F (2011) Factors affecting patterns of tick parasitism on forest rodents in tick-borne encephalitis risk areas, germany. Parasitol Res 108: 323-335. 


\section{References}

Kimura M and Ohta T (1969) The average number of generations until fixation of a mutant gene in a finite population. Genetics 61: 763-771.

Kipp S, Goedecke A, Dorn W, Wilske B and Fingerle V (2006) Role of birds in thuringia, germany, in the natural cycle of Borrelia burgdorferi sensu lato, the lyme disease spirochaete. Int J Med Microbiol 296 Suppl 40: 125-128.

Kitron U and Kazmierczak JJ (1997) Spatial analysis of the distribution of lyme disease in wisconsin. American journal of epidemiology 145: 558-566.

Kjelland V, Ytrehus B, Stuen S, Skarpaas T and Slettan A (2011) Prevalence of Borrelia burgdorferi in Ixodes ricinus ticks collected from moose (alces alces) and roe deer (capreolus capreolus) in southern norway. Ticks Tick Borne Dis 2: 99-103.

Kjemtrup AM and Conrad PA (2000) Human babesiosis: An emerging tick-borne disease. Int J Parasitol 30: 1323-1337.

Kobryn K and Chaconas G (2005) Fusion of hairpin telomeres by the B. burgdorferi telomere resolvase rest implications for shaping a genome in flux. Mol Cell 17: 783-791.

Koci J, Movila A, Taragel'ova V, Toderas I, Uspenskaia I, Derdakova M and Labuda M (2007) First report of Anaplasma phagocytophilum and its co-infections with Borrelia burgdorferi sensu lato in Ixodes ricinus ticks (acari: Ixodidae) from republic of moldova. Exp Appl Acarol 41: 147-152.

Koetsveld J, Tijsse-Klasen E, Herremans T, Hovius JW and Sprong H (2015) Serological and molecular evidence for spotted fever group rickettsia and Borrelia burgdorferi sensu lato co-infections in the netherlands. Ticks and tick-borne diseases.

Koetsveld J, Tijsse-Klasen E, Herremans T, Hovius JW and Sprong H (2016) Serological and molecular evidence for spotted fever group rickettsia and Borrelia burgdorferi sensu lato co-infections in the netherlands. Ticks Tick Borne Dis 7: 371-377.

Kuiper H, van Dam AP, Spanjaard L, de Jongh BM, Widjojokusumo A, Ramselaar TC, Cairo I, Vos K and Dankert J (1994) Isolation of Borrelia burgdorferi from biopsy specimens taken from healthy-looking skin of patients with lyme borreliosis. Journal of clinical microbiology 32: 715-720.

Kurtenbach K, De Michelis S, Etti S, Schafer SM, Sewell HS, Brade V and Kraiczy P (2002a) Host association of Borrelia burgdorferi sensu lato--the key role of host complement. Trends Microbiol 10: 74-79.

Kurtenbach K, De Michelis S, Etti S, Schafer SM, Sewell HS, Brade V and Kraiczy P (2002b) Host association of Borrelia burgdorferi sensu lato-the key role of host complement. Trends Microbiol 10: 74.

Kurtenbach K, Dizij A, Seitz HM, Margos G, Moter SE, Kramer MD, Wallich R, Schaible UE and Simon MM (1994) Differential immune responses to Borrelia burgdorferi in european wild rodent species influence spirochete transmission to Ixodes ricinus I. (acari: Ixodidae). Infect Immun 62: 5344-5352. 
Kurtenbach K, Hanincova K, Tsao Jl, Margos G, Fish D and Ogden NH (2006a) Fundamental processes in the evolutionary ecology of lyme borreliosis. Nat Rev Microbiol 4: 660-669.

Kurtenbach K, Hanincová K, Tsao Jl, Margos G, Fish D and Ogden NH (2006b) Fundamental processes in the evolutionary ecology of lyme borreliosis. Nat Rev Micro 4: 660-669.

Kurtenbach K, Kampen H, Dizij A, Arndt S, Seitz HM, Schaible UE and Simon MM (1995) Infestation of rodents with larval Ixodes ricinus (acari: Ixodidae) is an important factor in the transmission cycle of Borrelia burgdorferi s.L. In german woodlands. J Med Entomol 32: 807-817.

Kurtenbach K, Peacey M, Rijpkema SG, Hoodless AN, Nuttall PA and Randolph SE (1998) Differential transmission of the genospecies of Borrelia burgdorferi sensu lato by game birds and small rodents in england. Appl Environ Microbiol 64: 1169-1174.

Lee KA (2006) Linking immune defenses and life history at the levels of the individual and the species. Integrative and Comparative Biology 46: 1000-1015.

Lee KA, Wikelski M, Robinson WD, Robinson TR and Klasing KC (2008) Constitutive immune defences correlate with life-history variables in tropical birds. J Anim Ecol 77: 356-363.

Lesnik OM, Istomina O, Rijpkema S, Bruininck H and Beliaeva ML (1997) [the clinical manifestations of lyme borreliosis in the middle urals and their association with Borrelia burgdorferi genospecies]. Ter Arkh 69: 9-12.

Levene $H$ (1953) Genetic equilibrium when more than one ecological niche is available. The American Naturalist 87: 331-333.

Lindgren E and Jaenson DG (2006) Lyme borreliosis in europe: Influences of climate and climate change, epidemiology, ecology and adaptation measures, World Health Organisation Europe.

LoGiudice K, Duerr ST, Newhouse MJ, Schmidt KA, Killilea ME and Ostfeld RS (2008) Impact of host community composition on lyme disease risk. Ecology 89: 28412849.

LoGiudice K, Ostfeld RS, Schmidt KA and Keesing F (2003) The ecology of infectious disease: Effects of host diversity and community composition on lyme disease risk. Proc Natl Acad Sci U S A 100: 567-571.

Lommano E, Bertaiola L, Dupasquier C and Gern L (2012) Infections and coinfections of questing Ixodes ricinus ticks by emerging zoonotic pathogens in western switzerland. Applied and environmental microbiology 78: 4606-4612.

Lotric-Furlan S, Petrovec M, Avsic-Zupanc T, Nicholson WL, Sumner JW, Childs JE and Strle $F(1998)$ Human ehrlichiosis in central europe. Wien Klin Wochenschr 110: 894-897.

Lunemann JD, Zarmas S, Priem S, Franz J, Zschenderlein R, Aberer E, Klein R, Schouls L, Burmester GR and Krause A (2001) Rapid typing of Borrelia burgdorferi sensu lato 


\section{References}

species in specimens from patients with different manifestations of lyme borreliosis. J Clin Microbiol 39: 1130-1133.

Majlathova V, Majlath I, Derdakova M, Vichova B and Pet'ko B (2006) Borrelia lusitaniae and green lizards (lacerta viridis), karst region, slovakia. Emerging infectious diseases 12: 1895-1901.

Mannelli A, Bertolotti L, Gern L and Gray J (2012) Ecology of Borrelia burgdorferi sensu lato in europe: Transmission dynamics in multi-host systems, influence of molecular processes and effects of climate change. FEMS Microbiol Rev 36: 837861.

Mannelli A, Nebbia P, Tramuta C, Grego E, Tomassone L, Ainardi R, Venturini L, De Meneghi D and Meneguz PG (2005) Borrelia burgdorferi sensu lato infection in larval Ixodes ricinus (acari: Ixodidae) feeding on blackbirds in northwestern italy. J Med Entomol 42: 168-175.

Margos G, Chu CY, Takano A, Jiang BG, Liu W, Kurtenbach K, Masuzawa T, Fingerle V, Cao WC and Kawabata H (2015) Borrelia yangtzensis sp. Nov. A rodent associated species in asia is related to $B$. valaisiana. Int J Syst Evol Microbiol.

Margos G, Gatewood AG, Aanensen DM, Hanincova K, Terekhova D, Vollmer SA, Cornet M, Piesman J, Donaghy M, Bormane A, Hurn MA, Feil EJ, Fish D, Casjens S, Wormser GP, Schwartz I and Kurtenbach K (2008) Mlst of housekeeping genes captures geographic population structure and suggests a european origin of Borrelia burgdorferi. Proceedings of the National Academy of Sciences of the United States of America 105: 8730-8735.

Margos G, Vollmer SA, Cornet M, Garnier M, Fingerle V, Wilske B, Bormane A, Vitorino L, Collares-Pereira M, Drancourt $M$ and Kurtenbach K (2009) A new borrelia species defined by multilocus sequence analysis of housekeeping genes. Applied and environmental microbiology 75: 5410-5416.

Margos G, Vollmer SA, Ogden NH and Fish D (2011) Population genetics, taxonomy, phylogeny and evolution of Borrelia burgdorferi sensu lato. Infect Genet Evol 11: 1545-1563.

Margos G, Wilske B, Sing A, Hizo-Teufel C, Cao WC, Chu C, Scholz H, Straubinger RK and Fingerle V (2013) Borrelia bavariensis sp. Nov. Is widely distributed in europe and asia. Int J Syst Evol Microbiol 63: 4284-4288.

Marsot M, Chapuis JL, Gasqui P, Dozieres A, Masseglia S, Pisanu B, Ferquel E and Vourc'h $\mathrm{G}$ (2013) Introduced siberian chipmunks (tamias sibiricus barberi) contribute more to lyme borreliosis risk than native reservoir rodents. PLoS One 8: e55377.

Marsot M, Henry PY, Vourc'h G, Gasqui P, Ferquel E, Laignel J, Grysan M and Chapuis JL (2012) Which forest bird species are the main hosts of the tick, Ixodes ricinus, the vector of Borrelia burgdorferi sensu lato, during the breeding season? Int J Parasitol 42: 781-788.

Marsot M, Sigaud M, Chapuis JL, Ferquel E, Cornet M and Vourc'h G (2011) Introduced siberian chipmunks (tamias sibiricus barberi) harbor more-diverse Borrelia 
burgdorferi sensu lato genospecies than native bank voles (Myodes glareolus). Appl Environ Microbiol 77: 5716-5721.

Martello E, Mannelli A, Ragagli C, Ambrogi C, Selmi M, Ceballos LA and Tomassone L (2014) Range expansion of Ixodes ricinus to higher altitude, and co-infestation of small rodents with dermacentor marginatus in the northern apennines, italy. Ticks Tick Borne Dis 5: 970-974.

Martin D and Rybicki E (2000) Rdp: Detection of recombination amongst aligned sequences. Bioinformatics 16: 562-563.

Martin L, Hasselquist D and Wikelski M (2006) Investment in immune defense is linked to pace of life in house sparrows. Oecologia 147: 565-575.

Martin LB, 2nd, Weil ZM and Nelson RJ (2007) Immune defense and reproductive pace of life in peromyscus mice. Ecology 88: 2516-2528.

Masuzawa T, Kharitonenkov IG, Kadosaka T, Hashimoto N, Kudeken M, Takada N, Kaneda $\mathrm{K}$ and Imai Y (2005) Characterization of Borrelia burgdorferi sensu lato isolated in moscow province--a sympatric region for Ixodes ricinus and ixodes persulcatus. Int J Med Microbiol 294: 455-464.

Masuzawa T, Komikado T, Iwaki A, Suzuki H, Kaneda K and Yanagihara Y (1996a) Characterization of borrelia sp. Isolated from ixodes tanuki, i. Turdus, and i. Columnae in japan by restriction fragment length polymorphism of rrf $(5 \mathrm{~s})$-rrl (23s) intergenic spacer amplicons. FEMS Microbiol Lett 142: 77-83.

Masuzawa T, Suzuki H, Kawabata H, Ishiguro F, Takada N and Yanagihara Y (1996b) Characterization of borrelia spp. Isolated from the tick, ixodes tanuki and small rodents in japan. J Wildl Dis 32: 565-571.

Mather TN, Wilson ML, Moore SI, Ribeiro JM and Spielman A (1989) Comparing the relative potential of rodents as reservoirs of the lyme disease spirochete (Borrelia burgdorferi). Am J Epidemiol 130: 143-150.

Matuschka FR, Eiffert H, Ohlenbusch A and Spielman A (1994) Amplifying role of edible dormice in lyme disease transmission in central europe. J Infect Dis 170: 122-127.

Matuschka FR, Fischer P, Musgrave K, Richter D and Spielman A (1991) Hosts on which nymphal Ixodes ricinus most abundantly feed. Am J Trop Med Hyg 44: 100-107.

Matuschka FR, Heiler M, Eiffert H, Fischer P, Lotter H and Spielman A (1993) Diversionary role of hoofed game in the transmission of lyme disease spirochetes. Am J Trop Med Hyg 48: 693-699.

Matuschka FR, Lange R, Spielman A, Richter D and Fischer P (1990) Subadult Ixodes ricinus (acari: Ixodidae) on rodents in berlin, west germany. J Med Entomol 27: 385-390.

Mechai S, Margos G, Feil EJ, Barairo N, Lindsay LR, Michel P and Ogden NH (2016) Evidence for host-genotype associations of Borrelia burgdorferi sensu stricto. PLoS One 11: e0149345. 


\section{References}

Medlock JM, Hansford KM, Bormane A, Derdakova M, Estrada-Pena A, George JC, Golovljova I, Jaenson TG, Jensen JK, Jensen PM, Kazimirova M, Oteo JA, Papa A, Pfister K, Plantard O, Randolph SE, Rizzoli A, Santos-Silva MM, Sprong H, Vial L, Hendrickx G, Zeller H and Van Bortel W (2013) Driving forces for changes in geographical distribution of Ixodes ricinus ticks in europe. Parasit Vectors 6: 1.

Mejlon HA (1997) Diel activity of Ixodes ricinus acari:Ixodidae at two locations near stockholm, sweden. Exp Appl Acarol 21: 247-255.

Mejlon HA and Jaenson TGT (1997) Questing behaviour of Ixodes ricinus ticks (acari: Ixodidae). Experimental \& Applied Acarology 21: 747-754.

Michalik J, Hofman T, Buczek A, Skoracki M and Sikora B (2003) Borrelia burgdorferi s.L. In Ixodes ricinus (acari: Ixodidae) ticks collected from vegetation and small rodents in recreational areas of the city of poznan. J Med Entomol 40: 690-697.

Michalik J, Skotarczak B, Skoracki M, Wodecka B, Sikora B, Hofman T, Rymaszewska A and Sawczuk M (2005) Borrelia burgdorferi sensu stricto in yellow-necked mice and feeding Ixodes ricinus ticks in a forest habitat of west central poland. J Med Entomol 42: 850-856.

Michalik J, Wodecka B, Skoracki M, Sikora B and Stańczak J (2008) Prevalence of avianassociated Borrelia burgdorferi s.L. Genospecies in Ixodes ricinus ticks collected from blackbirds (turdus merula) and song thrushes (t. Philomelos). International Journal of Medical Microbiology 298, Supplement 1: 129-138.

Miyamoto K and Masuzawa T (2002) Ecology of Borrelia burgdorferi sensu lato in japan and east asia. In: J. Gray OK, R.s.Lane and G. Stanek,ed. Lyme borreliosis: Biology, epidemiology and control, CABI International.

Mogl C, de Mendonça PG, Harsch A and Heyl J (2011) Potential for cofeeding transmission of tick-borne pathogens in common voles and roe deer - supportive molecular evidence from field samples. Acta Zoologica Bulgarica 63: 6.

Moran Cadenas F, Rais O, Humair PF, Douet V, Moret J and Gern L (2007) Identification of host bloodmeal source and Borrelia burgdorferi sensu lato in field-collected Ixodes ricinus ticks in chaumont (switzerland). J Med Entomol 44: 1109-1117.

Nicholson MC and Mather TN (1996) Methods for evaluating lyme disease risks using geographic information systems and geospatial analysis. Journal of medical entomology 33: 711-720.

Niethammer J and Krapp F (1978) Handbuch der säugetiere europas, Handbuch der säugetiere europas, Aula Verlag.

Nieto NC and Foley JE (2009) Meta-analysis of coinfection and coexposure with Borrelia burgdorferi and Anaplasma phagocytophilum in humans, domestic animals, wildlife, and Ixodes ricinus-complex ticks. Vector borne and zoonotic diseases (Larchmont, NY) 9: 93-102.

Norte AC, Araujo PM, da Silva LP, Tenreiro PQ, Ramos JA, Nuncio MS, Ze-Ze L and de Carvalho IL (2015) Characterization through multilocus sequence analysis of borrelia turdi isolates from portugal. Microb Ecol. 
Ogden NH and Tsao Jl (2009) Biodiversity and lyme disease: Dilution or amplification? Epidemics 1: 196-206.

Oliver JH, Jr., Lin T, Gao L, Clark KL, Banks CW, Durden LA, James AM and Chandler FW, Jr. (2003) An enzootic transmission cycle of lyme borreliosis spirochetes in the southeastern united states. Proceedings of the National Academy of Sciences of the United States of America 100: 11642-11645.

Olsen B, Jaenson TGT, Noppa L, Bunikis J and Bergstrom S (1993) A lyme borreliosis cycle in seabirds and ixodes uriae ticks. Nature 362: 340-342.

Ostfeld RS (1985) Limiting resources and territoriality in microtine rodents. American Naturalist: 1-15.

Ostfeld RS and Holt RD (2004) Are predators good for your health? Evaluating evidence for top-down regulation of zoonotic disease reservoirs. Frontiers in Ecology and the Environment 2: 13-20.

Ostfeld RS and Keesing F (2000) Biodiversity and disease risk: The case of lyme disease

biodiversidad y riesgo de enfermedades: El caso de la enfermedad de lyme. Conservation Biology 14: 722-728.

Ostfeld RS, Levi T, Jolles AE, Martin LB, Hosseini PR and Keesing F (2014) Life history and demographic drivers of reservoir competence for three tick-borne zoonotic pathogens. PLoS One 9: e107387.

Pacilly FC, Benning ME, Jacobs F, Leidekker J, Sprong $\mathrm{H}$, Van Wieren SE and Takken W (2014) Blood feeding on large grazers affects the transmission of Borrelia burgdorferi sensu lato by Ixodes ricinus. Ticks Tick Borne Dis 5: 810-817.

Pal U, de Silva AM, Montgomery RR, Fish D, Anguita J, Anderson JF, Lobet $\mathrm{Y}$ and Fikrig E (2000) Attachment of Borrelia burgdorferi within ixodes scapularis mediated by outer surface protein a. J Clin Invest 106: 561-569.

Pal U and Fikrig E (2003) Adaptation of Borrelia burgdorferi in the vector and vertebrate host. Microbes and infection / Institut Pasteur 5: 659-666.

Pal U, Li X, Wang T, Montgomery RR, Ramamoorthi N, Desilva AM, Bao F, Yang X, Pypaert M, Pradhan D, Kantor FS, Telford S, Anderson JF and Fikrig E (2004) Trospa, an ixodes scapularis receptor for Borrelia burgdorferi. Cell 119: 457-468.

Parola P and Raoult D (2001) Ticks and tickborne bacterial diseases in humans: An emerging infectious threat. Clin Infect Dis 32: 897-928.

Pato FJ, Panadero R, Vazquez L, Lopez CM, Diaz P, Vazquez E, Diez-Banos P, Morrondo P and Fernandez $G$ (2013) Seroprevalence of Borrelia burgdorferi sensu lato in roe deer (capreolus capreolus) from northwestern spain. J Zoo Wildl Med 44: 660665.

Patterson N, Price AL and Reich D (2006) Population structure and eigenanalysis. PLoS genetics 2: e190.

Pausa M, Pellis V, Cinco M, Giulianini PG, Presani G, Perticarari S, Murgia R and Tedesco F (2003) Serum-resistant strains of Borrelia burgdorferi evade complement- 
mediated killing by expressing a cd59-like complement inhibitory molecule. J Immunol 170: 3214-3222.

Pecchioli E, Hauffe HC, Tagliapietra V, Bandi C, Genchi C and Rizzoli A (2007) Genospecies of Borrelia burgdorferi sensu lato in Ixodes ricinus ticks from the autonomous province of trento, italy. Int J Med Microbiol 297: 53-59.

Perez D, Kneubuhler Y, Rais O and Gern L (2012) Seasonality of Ixodes ricinus ticks on vegetation and on rodents and Borrelia burgdorferi sensu lato genospecies diversity in two lyme borreliosis-endemic areas in switzerland. Vector Borne Zoonotic Dis 12: 633-644.

Perez D, Kneubuhler Y, Rais O, Jouda F and Gern L (2011) Borrelia afzelii ospc genotype diversity in Ixodes ricinus questing ticks and ticks from rodents in two lyme borreliosis endemic areas: Contribution of co-feeding ticks. Ticks Tick Borne Dis 2: 137-142.

Perkins SE, Cattadori IM, Tagliapietra V, Rizzoli AP and Hudson PJ (2003) Empirical evidence for key hosts in persistence of a tick-borne disease. Int J Parasitol 33: 909-917.

Pfaffle M, Littwin N, Muders SV and Petney TN (2013) The ecology of tick-borne diseases. Int J Parasitol 43: 1059-1077.

Pfaffle M, Petney T, Skuballa J and Taraschewski H (2011) Comparative population dynamics of a generalist (Ixodes ricinus) and specialist tick (i. Hexagonus) species from european hedgehogs. Exp Appl Acarol 54: 151-164.

Pichon B, Gilot B and Perez-Eid C (2000) Detection of spirochaetes of Borrelia burgdorferi complexe in the skin of cervids by pcr and culture. Eur J Epidemiol 16: 869-873.

Piesman J (2002) Ecology of Borrelia burgdorferi sensu lato in north america. In: J. Gray OK, R.s.Lane and G. Stanek,ed. Lyme borreliosis: Biology, epidemiology and control, CABI International.

Piesman J and Gern L (2004a) Lyme borreliosis in europe and north america. Parasitology 129: S191-S220.

Piesman J and Gern L (2004b) Lyme borreliosis in europe and north america. Parasitology 129 Suppl: S191-220.

Piesman J and Sinsky RJ (1988) Ability to ixodes scapularis, dermacentor variabilis, and amblyomma americanum (acari: Ixodidae) to acquire, maintain, and transmit lyme disease spirochetes (Borrelia burgdorferi). J Med Entomol 25: 336-339.

Pisanu B, Chapuis JL, Dozieres A, Basset F, Poux V and Vourc'h G (2014) High prevalence of Borrelia burgdorferi s.L. In the european red squirrel sciurus vulgaris in france. Ticks Tick Borne Dis 5: 1-6.

Platonov AE, Karan LS, Kolyasnikova NM, Makhneva NA, Toporkova MG, Maleev VV, Fish D and Krause PJ (2011) Humans infected with relapsing fever spirochete Borrelia miyamotoi, russia. Emerg Infect Dis 17: 1816-1823. 
Posey JE and Gherardini FC (2000) Lack of a role for iron in the lyme disease pathogen. Science 288: 1651-1653.

Previtali MA, Ostfeld RS, Keesing F, Jolles AE, Hanselmann R and Martin LB (2012) Relationship between pace of life and immune responses in wild rodents. Oikos 121: 1483-1492.

Purser JE, Lawrenz MB, Caimano MJ, Howell JK, Radolf JD and Norris SJ (2003) A plasmidencoded nicotinamidase (pnca) is essential for infectivity of Borrelia burgdorferi in a mammalian host. Mol Microbiol 48: 753-764.

Qiu WG, Dykhuizen DE, Acosta MS and Luft BJ (2002) Geographic uniformity of the lyme disease spirochete (Borrelia burgdorferi) and its shared history with tick vector (ixodes scapularis) in the northeastern united states. Genetics 160: 833-849.

R_Core_Team (2015) R: A language and environment for statistical computing, R Foundation for Statistical Computing, Vienna, Austria.

Raberg L (2012) Infection intensity and infectivity of the tick-borne pathogen Borrelia afzelii. J Evol Biol 25: 1448-1453.

Radolf JD, Caimano MJ, Stevenson B and Hu LT (2012) Of ticks, mice and men: Understanding the dual-host lifestyle of lyme disease spirochaetes. Nat Rev Microbiol 10: 87-99.

Radzijevskaja J, Paulauskas A, Rosef O, Petkevicius S, Mazeika V and Rekasius T (2013) The propensity of voles and mice to transmit Borrelia burgdorferi sensu lato infection to feeding ticks. Vet Parasitol 197: 318-325.

Randolph SE (2001) The shifting landscape of tick-borne zoonoses: Tick-borne encephalitis and lyme borreliosis in europe. Philos Trans R Soc Lond B Biol Sci 356: 1045-1056.

Randolph SE (2004) Tick ecology: Processes and patterns behind the epidemiological risk posed by ixodid ticks as vectors. Parasitology 129 Suppl: S37-65.

Randolph SE and Dobson AD (2012) Pangloss revisited: A critique of the dilution effect and the biodiversity-buffers-disease paradigm. Parasitology 139: 847-863.

Rauter C and Hartung T (2005) Prevalence of Borrelia burgdorferi sensu lato genospecies in Ixodes ricinus ticks in europe: A metaanalysis. Applied and environmental microbiology 71: 7203-7216.

Raveche ES, Schutzer SE, Fernandes H, Bateman H, McCarthy BA, Nickell SP and Cunningham MW (2005) Evidence of borrelia autoimmunity-induced component of lyme carditis and arthritis. J Clin Microbiol 43: 850-856.

Reik L, Jr., Burgdorfer W and Donaldson JO (1986) Neurologic abnormalities in lyme disease without erythema chronicum migrans. The American journal of medicine 81: 73-78.

Reye AL, Hubschen JM, Sausy A and Muller CP (2010) Prevalence and seasonality of tickborne pathogens in questing Ixodes ricinus ticks from luxembourg. Applied and environmental microbiology 76: 2923-2931. 


\section{References}

Richter D, Debski A, Hubalek Z and Matuschka FR (2012) Absence of lyme disease spirochetes in larval Ixodes ricinus ticks. Vector Borne Zoonotic Dis 12: 21-27.

Richter D and Matuschka FR (2006) Perpetuation of the lyme disease spirochete Borrelia lusitaniae by lizards. Appl Environ Microbiol 72: 4627-4632.

Richter D, Schlee DB, Allgower R and Matuschka FR (2004) Relationships of a novel lyme disease spirochete, borrelia spielmani sp. Nov., with its hosts in central europe. Appl Environ Microbiol 70: 6414-6419.

Richter D, Schlee DB and Matuschka FR (2003) Relapsing fever-like spirochetes infecting european vector tick of lyme disease agent. Emerg Infect Dis 9: 697-701.

Richter D, Schlee DB and Matuschka FR (2011) Reservoir competence of various rodents for the lyme disease spirochete Borrelia spielmanii. Applied and environmental microbiology 77: 3565-3570.

Rijpkema SG, Tazelaar DJ, Molkenboer MJ, Noordhoek GT, Plantinga G, Schouls LM and Schellekens JF (1997) Detection of Borrelia afzelii, Borrelia burgdorferi sensu stricto, Borrelia garinii and group vs116 by pcr in skin biopsies of patients with erythema migrans and acrodermatitis chronica atrophicans. Clinical microbiology and infection : the official publication of the European Society of Clinical Microbiology and Infectious Diseases 3: 109-116.

Rollend L, Fish D and Childs JE (2013) Transovarial transmission of borrelia spirochetes by ixodes scapularis: A summary of the literature and recent observations. Ticks Tick Borne Dis 4: 46-51.

Rozsa L, Reiczigel J and Majoros G (2000) Quantifying parasites in samples of hosts. J Parasitol 86: 228.

Ruyts SC, Ampoorter E, Coipan EC, Baeten L, Heylen D, Sprong H, Matthysen E and Verheyen K (2016) Diversifying forest communities may change lyme disease risk: Extra dimension to the dilution effect in europe. Parasitology: 1-10.

Rydkina E, Roux V, Rudakov N, Gafarova M, Tarasevich I and Raoult D (1999) New rickettsiae in ticks collected in territories of the former soviet union. Emerg Infect Dis 5: 811-814.

Ryffel K, Peter O, Rutti B, Suard A and Dayer E (1999) Scored antibody reactivity determined by immunoblotting shows an association between clinical manifestations and presence of Borrelia burgdorferi sensu stricto, B. garinii, $B$. afzelii, and B. valaisiana in humans. J Clin Microbiol 37: 4086-4092.

Salzburger W, Ewing GB and Von Haeseler A (2011) The performance of phylogenetic algorithms in estimating haplotype genealogies with migration. Mol Ecol 20: 19521963.

Sanger F, Nicklen S and Coulson AR (1977) DNA sequencing with chain-terminating inhibitors. Proc Natl Acad Sci U S A 74: 5463-5467.

Schaarschmidt D, Oehme R, Kimmig P, Hesch RD and Englisch S (2001) Detection and molecular typing of Borrelia burgdorferi sensu lato in Ixodes ricinus ticks and in 
different patient samples from southwest germany. Eur J Epidemiol 17: 10671074.

Schlitter D, van der Straeten E, Amori G, Hutterer R, Kryštufek B, Yigit N and Mitsain G (2008) Apodemus sylvaticus. Available at: http://dx.doi.org/10.2305/IUCN.UK.2008.RLTS.T1904A8791394.en. Accessed 15 Nov.

Schmidt S, Essbauer SS, Mayer-Scholl A, Poppert S, Schmidt-Chanasit J, Klempa B, Henning K, Schares G, Groschup MH, Spitzenberger F, Richter D, Heckel G and Ulrich RG (2014) Multiple infections of rodents with zoonotic pathogens in austria. Vector Borne Zoonotic Dis 14: 467-475.

Schouls LM, Van De Pol I, Rijpkema SG and Schot CS (1999) Detection and identification of ehrlichia, Borrelia burgdorferi sensu lato, and bartonella species in dutch Ixodes ricinus ticks. J Clin Microbiol 37: 2215-2222.

Schroder NW, Diterich I, Zinke A, Eckert J, Draing C, von Baehr V, Hassler D, Priem S, Hahn K, Michelsen KS, Hartung T, Burmester GR, Gobel UB, Hermann C and Schumann RR (2005) Heterozygous arg753gln polymorphism of human tIr-2 impairs immune activation by Borrelia burgdorferi and protects from late stage lyme disease. J Immunol 175: 2534-2540.

Schutzer SE, Fraser-Liggett CM, Casjens SR, Qiu WG, Dunn JJ, Mongodin EF and Luft BJ (2011) Whole-genome sequences of thirteen isolates of Borrelia burgdorferi. J Bacteriol 193: 1018-1020.

Schutzer SE, Fraser-Liggett CM, Qiu WG, Kraiczy P, Mongodin EF, Dunn JJ, Luft BJ and Casjens SR (2012) Whole-genome sequences of borrelia bissettii, Borrelia valaisiana, and Borrelia spielmanii. J Bacteriol 194: 545-546.

Schwan TG and Piesman J (2000) Temporal changes in outer surface proteins a and c of the lyme disease-associated spirochete, Borrelia burgdorferi, during the chain of infection in ticks and mice. J Clin Microbiol 38: 382-388.

Scott MC, Rosen ME, Hamer SA, Baker E, Edwards H, Crowder C, Tsao JI and Hickling GJ (2010) High-prevalence Borrelia miyamotoi infection among [corrected] wild turkeys (meleagris gallopavo) in tennessee. J Med Entomol 47: 1238-1242.

Severinsson K, Jaenson TG, Pettersson J, Falk K and Nilsson K (2010) Detection and prevalence of Anaplasma phagocytophilum and rickettsia helvetica in Ixodes ricinus ticks in seven study areas in sweden. Parasit Vectors 3: 66.

Silaghi C, Beck R, Oteo JA, Pfeffer M and Sprong H (2016) Neoehrlichiosis: An emerging tick-borne zoonosis caused by candidatus Neoehrlichia mikurensis. Exp Appl Acarol 68: 279-297.

Silaghi C, Woll D, Hamel D, Pfister K, Mahling M and Pfeffer M (2012a) Babesia spp. And Anaplasma phagocytophilum in questing ticks, ticks parasitizing rodents and the parasitized rodents--analyzing the host-pathogen-vector interface in a metropolitan area. Parasit Vectors 5: 191. 


\section{References}

Silaghi C, Woll D, Mahling M, Pfister K and Pfeffer M (2012b) Candidatus Neoehrlichia mikurensis in rodents in an area with sympatric existence of the hard ticks Ixodes ricinus and dermacentor reticulatus, germany. Parasit Vectors 5: 285.

Singh SK and Girschick HJ (2004) Lyme borreliosis: From infection to autoimmunity. Clin Microbiol Infect 10: 598-614.

Sinski E, Bajer A, Welc R, Pawelczyk A, Ogrzewalska M and Behnke JM (2006a) Babesia microti: Prevalence in wild rodents and Ixodes ricinus ticks from the mazury lakes district of north-eastern poland. Int J Med Microbiol 296 Suppl 40: 137-143.

Sinski E, Pawelczyk A, Bajer A and Behnke J (2006b) Abundance of wild rodents, ticks and environmental risk of lyme borreliosis: A longitudinal study in an area of mazury lakes district of poland. Ann Agric Environ Med 13: 295-300.

Slatkin M (1991) Inbreeding coefficients and coalescence times. Genet Res 58: 167-175.

Slatkin M (1994) An exact test for neutrality based on the ewens sampling distribution. Genet Res 64: 71-74.

Smith JM and Haigh J (1974) The hitch-hiking effect of a favourable gene. Genet Res 23: 23-35.

Smith R and Takkinen J (2006) Lyme borreliosis: Europe-wide coordinated surveillance and action needed? Euro Surveill 11: E060622 060621.

Sprong H, Hofhuis A, Gassner F, Takken W, Jacobs F, van Vliet AJ, van Ballegooijen M, van der Giessen J and Takumi K (2012a) Circumstantial evidence for an increase in the total number and activity of borrelia-infected Ixodes ricinus in the netherlands. Parasit Vectors 5: 294.

Sprong H, Tijsse-Klasen E, Langelaar M, De Bruin A, Fonville M, Gassner F, Takken W, Van Wieren S, Nijhof A, Jongejan F, Maassen CB, Scholte EJ, Hovius JW, Emil Hovius K, Spitalska E and Van Duynhoven YT (2012b) Prevalence of coxiella burnetii in ticks after a large outbreak of q fever. Zoonoses Public Health 59: 69-75.

Sprong $H$, Wielinga PR, Fonville M, Reusken C, Brandenburg AH, Borgsteede F, Gaasenbeek C and van der Giessen JW (2009) Ixodes ricinus ticks are reservoir hosts for rickettsia helvetica and potentially carry flea-borne rickettsia species. Parasit Vectors 2: 41.

Stanek G, Wormser GP, Gray J and Strle F (2012) Lyme borreliosis. Lancet 379: 461-473.

States SL, Brinkerhoff RJ, Carpi G, Steeves TK, Folsom-O'Keefe C, DeVeaux M and DiukWasser MA (2014) Lyme disease risk not amplified in a species-poor vertebrate community: Similar Borrelia burgdorferi tick infection prevalence and ospc genotype frequencies. Infect Genet Evol 27: 566-575.

Steere AC, Gross D, Meyer AL and Huber BT (2001) Autoimmune mechanisms in antibiotic treatment-resistant lyme arthritis. J Autoimmun 16: 263-268.

Strle F, Picken RN, Cheng Y, Cimperman J, Maraspin V, Lotric-Furlan S, Ruzic-Sabljic E and Picken MM (1997) Clinical findings for patients with lyme borreliosis caused by Borrelia burgdorferi sensu lato with genotypic and phenotypic similarities to strain 
25015. Clinical infectious diseases : an official publication of the Infectious Diseases Society of America 25: 273-280.

Strle K, Jones KL, Drouin EE, Li X and Steere AC (2011) Borrelia burgdorferi rst1 (ospc type a) genotype is associated with greater inflammation and more severe lyme disease. The American journal of pathology 178: 2726-2739.

Stuen S, Granquist EG and Silaghi C (2013) Anaplasma phagocytophilum--a widespread multi-host pathogen with highly adaptive strategies. Frontiers in cellular and infection microbiology 3: 31.

Svendsen CB (2011) Is sarcoidosis a rickettsiosis? Dan Med Bull 58: B4249.

Swanson SJ, Neitzel D, Reed KD and Belongia EA (2006) Coinfections acquired from ixodes ticks. Clinical microbiology reviews 19: 708-727.

Takken W, Van Vliet AJ, Verhulst NO, Jacobs F, Gassner F, Hartemink N, Mulder S and Sprong $\mathrm{H}$ (in press) Acarological risk of Borrelia burgdorferi sensu lato infections across space and time in the netherlands. Vector Borne Zoonotic Dis.

Talleklint L and Jaenson TG (1994) Transmission of Borrelia burgdorferi s.L. From mammal reservoirs to the primary vector of lyme borreliosis, Ixodes ricinus (acari: Ixodidae), in sweden. J Med Entomol 31: 880-886.

Talleklint L and Jaenson TG (1996) Relationship between Ixodes ricinus density and prevalence of infection with borrelia-like spirochetes and density of infected ticks. J Med Entomol 33: 805-811.

Talleklint $L$ and Jaenson TG (1997) Infestation of mammals by Ixodes ricinus ticks (acari: Ixodidae) in south-central sweden. Exp Appl Acarol 21: 755-771.

Taragel'ova V, Koci J, Hanincova K, Kurtenbach K, Derdakova M, Ogden NH, Literak I, Kocianova E and Labuda M (2008) Blackbirds and song thrushes constitute a key reservoir of Borrelia garinii, the causative agent of borreliosis in central europe. Appl Environ Microbiol 74: 1289-1293.

Taylor KR, Takano A, Konnai S, Shimozuru M, Kawabata H and Tsubota T (2013) Borrelia miyamotoi infections among wild rodents show age and month independence and correlation with ixodes persulcatus larval attachment in hokkaido, japan. Vector Borne Zoonotic Dis 13: 92-97.

Thomas V, Anguita J, Barthold SW and Fikrig E (2001) Coinfection with Borrelia burgdorferi and the agent of human granulocytic ehrlichiosis alters murine immune responses, pathogen burden, and severity of lyme arthritis. Infect Immun 69: 3359-3371.

Tijsse-Klasen E, Braks M, Scholte EJ and Sprong H (2011a) Parasites of vectors-ixodiphagus hookeri and its wolbachia symbionts in ticks in the netherlands. Parasit Vectors 4: 228.

Tijsse-Klasen E, Fonville M, Gassner F, Nijhof AM, Hovius EK, Jongejan F, Takken W, Reimerink JR, Overgaauw PA and Sprong H (2011b) Absence of zoonotic bartonella species in questing ticks: First detection of bartonella clarridgeiae and rickettsia felis in cat fleas in the netherlands. Parasit Vectors 4: 61. 


\section{References}

Tijsse-Klasen E, Fonville M, Reimerink JH, Spitzen-van der Sluijs A and Sprong H (2010) Role of sand lizards in the ecology of lyme and other tick-borne diseases in the netherlands. Parasit Vectors 3: 42.

Tijsse-Klasen E, Jacobs JJ, Swart A, Fonville M, Reimerink JH, Brandenburg AH, van der Giessen JW, Hofhuis A and Sprong H (2011c) Small risk of developing symptomatic tick-borne diseases following a tick bite in the netherlands. Parasit Vectors 4: 17.

Tonetti N, Voordouw MJ, Durand J, Monnier S and Gern L (2015) Genetic variation in transmission success of the lyme borreliosis pathogen Borrelia afzelii. Ticks Tick Borne Dis 6: 334-343.

Toutoungi LN and Gern L (1993) Ability of transovarially and subsequent transstadially infected ixodes hexagonus ticks to maintain and transmit Borrelia burgdorferi in the laboratory. Exp Appl Acarol 17: 581-586.

Urwin R and Maiden MCJ (2003) Multi-locus sequence typing: A tool for global epidemiology. Trends in Microbiology 11: 479-487.

Vaclav R, Ficova M, Prokop P and Betakova T (2011) Associations between coinfection prevalence of Borrelia lusitaniae, anaplasma sp., and rickettsia sp. In hard ticks feeding on reptile hosts. Microb Ecol 61: 245-253.

van Dam AP, Kuiper H, Vos K, Widjojokusumo A, de Jongh BM, Spanjaard L, Ramselaar AC, Kramer MD and Dankert J (1993) Different genospecies of Borrelia burgdorferi are associated with distinct clinical manifestations of lyme borreliosis. Clinical infectious diseases : an official publication of the Infectious Diseases Society of America 17: 708-717.

van Dobbenburgh A, van Dam AP and Fikrig E (1999) Human granulocytic ehrlichiosis in western europe. N Engl J Med 340: 1214-1216.

van Duijvendijk G, Coipan C, Wagemakers A, Fonville M, Ersoz J, Oei A, Foldvari G, Hovius J, Takken W and Sprong H (2016) Larvae of Ixodes ricinus transmit Borrelia afzelii and B. miyamotoi to vertebrate hosts. Parasit Vectors 9: 97.

van Overbeek L, Gassner F, van der Plas CL, Kastelein P, Nunes-da Rocha U and Takken W (2008) Diversity of Ixodes ricinus tick-associated bacterial communities from different forests. FEMS Microbiol Ecol 66: 72-84.

van Strien AJ, Bekker DL, La Haye MJJ and van der Meij T (2015) Trends in small mammals derived from owl pellet data using occupancy modelling. Mammalian Biology - Zeitschrift für Säugetierkunde 80: 340-346.

Vannier E and Krause PJ (2012) Human babesiosis. New England Journal of Medicine 366: 2397-2407.

Vanwambeke SO, Sumilo D, Bormane A, Lambin EF and Randolph SE (2010) Landscape predictors of tick-borne encephalitis in latvia: Land cover, land use, and land ownership. Vector Borne Zoonotic Dis 10: 497-506.

Vazquez L, Panadero R, Dacal V, Pato FJ, Lopez C, Diaz P, Arias MS, Fernandez G, DiezBanos $P$ and Morrondo $P$ (2011) Tick infestation (acari: Ixodidae) in roe deer 
(capreolus capreolus) from northwestern spain: Population dynamics and risk stratification. Exp Appl Acarol 53: 399-409.

Vitorino LR, Margos G, Feil EJ, Collares-Pereira M, Ze-Ze L and Kurtenbach K (2008) Finescale phylogeographic structure of Borrelia lusitaniae revealed by multilocus sequence typing. PloS one 3: e4002.

Vollmer SA, Bormane A, Dinnis RE, Seelig F, Dobson AD, Aanensen DM, James MC, Donaghy M, Randolph SE, Feil EJ, Kurtenbach K and Margos G (2011) Host migration impacts on the phylogeography of lyme borreliosis spirochaete species in europe. Environmental microbiology 13: 184-192.

Vollmer SA, Feil EJ, Chu CY, Raper SL, Cao WC, Kurtenbach K and Margos G (2013) Spatial spread and demographic expansion of lyme borreliosis spirochaetes in eurasia. Infection, genetics and evolution : journal of molecular epidemiology and evolutionary genetics in infectious diseases 14: 147-155.

von Loewenich FD, Geissdorfer W, Disque C, Matten J, Schett G, Sakka SG and Bogdan C (2010) Detection of "candidatus Neoehrlichia mikurensis" in two patients with severe febrile illnesses: Evidence for a european sequence variant. J Clin Microbiol 48: 2630-2635.

Vor T, Kiffner C, Hagedorn P, Niedrig M and Ruhe F (2010) Tick burden on european roe deer (capreolus capreolus). Exp Appl Acarol 51: 405-417.

Vourc'h G, Marmet J, Chassagne M, Bord S and Chapuis JL (2007) Borrelia burgdorferi sensu lato in siberian chipmunks (tamias sibiricus) introduced in suburban forests in france. Vector Borne Zoonotic Dis 7: 637-641.

Wagemakers A, Koetsveld J, Narasimhan S, Wickel M, Deponte K, Bleijlevens B, Jahfari S, Sprong H, Karan LS, Sarksyan DS, van der Poll T, Bockenstedt LK, Bins AD, Platonov AE, Fikrig E and Hovius JW (2016) Variable major proteins as targets for specific antibodies against Borrelia miyamotoi. J Immunol 196: 4185-4195.

Wang G, van Dam AP and Dankert J (1999a) Phenotypic and genetic characterization of a novel Borrelia burgdorferi sensu lato isolate from a patient with lyme borreliosis. J Clin Microbiol 37: 3025-3028.

Wang G, van Dam AP, Schwartz I and Dankert J (1999b) Molecular typing of Borrelia burgdorferi sensu lato: Taxonomic, epidemiological, and clinical implications. Clin Microbiol Rev 12: 633-653.

Watterson GA (1977) Heterosis or neutrality? Genetics 85: 789-814.

Wegner Z, Stanczak J, Racewicz M, Kubica-Biernat B and Kruminis-Lozowska W (1997) The etiological agent of lyme disease, Borrelia burgdorferi, in ticks (acari:Ixodidae) from eastern poland. Zentralbl Bakteriol 286: 93-106.

Welc-Falęciak R, Bajer A, Behnke JM and Siński E (2008) Effects of host diversity and the community composition of hard ticks (ixodidae) on babesia microti infection. International Journal of Medical Microbiology 298, Supplement 1: 235-242. 
Welinder-Olsson C, Kjellin E, Vaht K, Jacobsson S and Wenneras C (2010) First case of human "candidatus Neoehrlichia mikurensis" infection in a febrile patient with chronic lymphocytic leukemia. J Clin Microbiol 48: 1956-1959.

Wielinga PR, Fonville M, Sprong H, Gaasenbeek C, Borgsteede F and van der Giessen JW (2009) Persistent detection of babesia eu1 and babesia microti in Ixodes ricinus in the netherlands during a 5-year surveillance: 2003-2007. Vector Borne Zoonotic Dis 9: 119-122.

Wielinga PR, Gaasenbeek C, Fonville M, de Boer A, de Vries A, Dimmers W, Akkerhuis Op Jagers G, Schouls LM, Borgsteede F and van der Giessen JW (2006) Longitudinal analysis of tick densities and borrelia, anaplasma, and ehrlichia infections of Ixodes ricinus ticks in different habitat areas in the netherlands. Appl Environ Microbiol 72: 7594-7601.

Wilhelmsson P, Fryland L, Borjesson S, Nordgren J, Bergstrom S, Ernerudh J, Forsberg P and Lindgren PE (2010) Prevalence and diversity of borrelia species in ticks that have bitten humans in sweden. J Clin Microbiol 48: 4169-4176.

Wilske B, Preac-Mursic V, Schierz G and Busch KV (1986) Immunochemical and immunological analysis of european Borrelia burgdorferi strains. Zentralbl Bakteriol Mikrobiol Hyg A 263: 92-102.

Wodecka B, Rymaszewska A and Skotarczak B (2014) Host and pathogen DNA identification in blood meals of nymphal Ixodes ricinus ticks from forest parks and rural forests of poland. Exp Appl Acarol 62: 543-555.

Wood CL and Lafferty KD (2013) Biodiversity and disease: A synthesis of ecological perspectives on lyme disease transmission. Trends Ecol Evol 28: 239-247.

Woolhouse ME, Taylor LH and Haydon DT (2001) Population biology of multihost pathogens. Science 292: 1109-1112.

Wormser GP, Brisson D, Liveris D, Hanincova K, Sandigursky S, Nowakowski J, Nadelman RB, Ludin S and Schwartz I (2008) Borrelia burgdorferi genotype predicts the capacity for hematogenous dissemination during early lyme disease. The Journal of infectious diseases 198: 1358-1364.

Wright $S$ (1943) Isolation by distance. Genetics 28: 114-138.

Xu G, Fang QQ, Keirans JE and Durden LA (2003) Molecular phylogenetic analyses indicate that the Ixodes ricinus complex is a paraphyletic group. J Parasitol 89: 452-457. 


\section{Summary}

Lyme borreliosis is the most prevalent vector-borne disease in the temperate regions of the northern hemisphere. The bacteria that cause it are members of the Borrelia burgdorferi sensu lato complex, a group of spirochaetes which are transmitted by hard ticks of the Ixodes ricinus complex. In several European countries, including The Netherlands, the incidence of Lyme borreliosis has been on the rise for the last decades. The acarological risk of human infection with Borrelia burgdorferi s.I. has been defined as the density of infected questing nymphs. This definition assumes that the distribution of the various genospecies of Borrelia in Lyme borreliosis is reflected in their distribution in questing ticks; furthermore, it assumes that all Borrelia genospecies are considered equally hazardous for humans. In order to define effective intervention strategies for controlling the disease, more insight in the transmission dynamics of tick-borne pathogens, both between animals and ticks, but also from ticks to humans is needed. As part of a Dutch research programme - "Shooting the messenger" - this PhD thesis focussed on linking the transmission cycles of Lyme spirochaetes to the different clinical manifestations of Lyme borreliosis. To that end, I explored aspects of the ecology and molecular adaptations of $B$. burgdorferi s.l. at various scales, from complex to genospecies level.

The ecological adaptations of $B$. burgdorferi s.l. are underpinned by a complex genomic structure and gene expression, with large genetic variation among and within the genospecies. In Chapter 3, we prove that the 5S-23S rDNA intergenic spacer (IGS) is a suitable molecular marker for identification of $B$. burgdorferi s.l. to genospecies level, but also to characterize the genetic diversity at intragenospecies level and to detect genetic differentiation among the subpopulations of Borrelia. Consequently, we used this marker in combination with other genetic markers, in the studies addressing the genetic diversity of Borrelia in small mammals and humans.

The main transmission route of these bacteria is the interstadial one, from larvae to nymphs and from nymphs to adult ticks. Larvae of $I$. ricinus can become infected during a blood meal from an infected host and during a blood meal in the vicinity of an infected nymph feeding on a host, a process known as cofeeding. The infected engorged larvae then moult into infected nymphs, which can transmit the spirochaetes to new hosts. The same process is repeated in the next developmental stage - nymph to adult. Thus, the maintenance of the bacteria in enzootic cycles is dependent on various species of vertebrates and 


\section{Summary}

the ticks that feed on them. In order to identify the main vertebrate hosts responsible for the maintenance of $B$. burgdorferi in enzootic cycles, but also for feeding $I$. ricinus ticks, we conducted a meta-analysis on literature data (Chapter 2). Our quantification method suggests that only a few host species, which are amongst the most widespread species in the environment (rodents, thrushes and deer), feed the majority of $I$. ricinus individuals and that rodents infect the majority of $I$. ricinus larvae with $B$. burgdorferi s.I.. The increase in distribution and abundance of these species, could be one of the main causes for the increase in Lyme borreliosis incidence in Europe in recent decades.

While at genospecies level, there is host specificity, with $B$. afzelii associated with rodents and $B$. garinii with birds, we wanted to see if the same holds true at intragenospecies level, for the various genotypes of Borrelia. Chapter 4 focuses on the rodents, which were identified in the literature metaanalysis as being the main hosts for I. ricinus larvae as well as for Borrelia afzelii. We tested the multiple niche polymorphisms hypothesis, using IGS, dbpA and ospC as molecular markers for typing $B$. afzelii genotypes in fed larvae collected from rodents in various areas in The Netherlands. Despite the high genetic diversity within $B$. afzelii, there was no difference between wood mice and bank voles in the number and types of $B$. afzelii haplotypes they transmit. Additionally, we compared the quantitative role of bank voles and wood mice in B. afzelii and Neoehrlichia mikurensis maintenance, another emerging tick-borne pathogen in Europe. Neoehrlichia mikurensis prevalence was positively associated with B. afzelii. Mathematical models including tick burden and infection prevalence indicated that bank voles are better amplifiers of these two bacteria than wood mice. Our study suggests that wood mice and bank voles differ in their contribution to the dynamics of $B$. afzelii, and possibly other TBP, in questing ticks but not in their contribution to the genetic diversity of these microorganisms.

The density of the vertebrate hosts and the feeding preferences of the ticks should determine the prevalence of $B$. burgdorferi s.l. genospecies in questing ticks. We address this topic in Chapter 5 , by testing 5,570 questing $I$. ricinus nymphs from 22 different areas in The Netherlands. We found an overall prevalence of $11.8 \%$ for $B$. burgdorferi s.l., with large and consistent variations among the various locations. As expected based on the results of Chapter 2, Borrelia afzelii was predominant (6.7 \% of the questing ticks) among the $B$. burgdorferi s.l. genospecies. It was followed by $B$. garinii/B. bavariensis (1.5\%), B. valaisiana (1.2\%), and B. burgdorferi sensu stricto (0.2\%). We noticed that, over the usual range of questing ticks' densities, the density of infected ticks is increasing with the overall density of questing ticks, and a downward trend might be observed only for questing tick densities of over 200/100 m2. This 
indicates that the density of questing nymphs is the main driver of the acarological risk of human exposure to B. burgdorferi s.l.

We also screened for the presence of other tick-borne pathogens that have previously been detected in questing ticks in The Netherlands: Rickettsia helvetica, Anaplasma phagocytophilum, Neoehrlichia mikurensis and several Babesia spp. (Chapter 5). To test whether these pathogens might share similar enzootic cycles we looked for patterns of coinfection and seasonal dynamics of infection in questing $I$. ricinus nymphs. One-third of the Borrelia-positive ticks were infected with at least one other pathogen. Coinfection of $B$. afzelii with $N$. mikurensis and with Babesia spp. occurred significantly more often than single infections, indicating the existence of mutual reservoir hosts. The diversity of tick-borne pathogens detected in $I$. ricinus in this study and the frequency of their coinfections with $B$. burgdorferi s.l. underline the need to consider them when evaluating the risks of infection and subsequently the risk of disease following a tick bite.

Chapter 6 addresses the pathogenicity of $B$. burgdorferi s.I. genospecies and genotypes for humans, using the eight multilocus sequence typing scheme housekeeping genes (MLST) and IGS as molecular markers. The frequency of the Borrelia spp. in humans is compared to the frequency in questing ticks to assess the infectivity of the various genospecies and genotypes. The fraction of STs that were isolated from human samples was significantly higher for the genospecies that are known to be maintained in enzootic cycles by mammals ( $B$. afzelii, $B$. bavariensis, and spielmanii) than for genospecies that are maintained by birds (B. garinii and B. valaisiana) or lizards ( $B$. lusitaniae). Just as in questing ticks, $B$. afzelii was the most prevalent Borrelia in in human Lyme borreliosis. Borrelia afzelii was associated with acrodermatitis chronica atrophicans, while $B$. garinii and $B$. bavariensis were associated with neuroborreliosis. Despite its high incidence in ticks and erythema migrans, in terms of disease burden (as measured by disability-adjusted life year), $B$. afzelii is of least concern for public health. Other Borrelia spirochaetes that are rarely found in questing I. ricinus ticks, such as $B$. bavariensis, seem to be responsible for most of the neuroborreliosis cases - a more severe clinical symptom of Lyme borreliosis. This implies that the prevalence of $B$. burgdorferi s.l. in questing ticks does not necessarily reflect the incidence of human Lyme borreliosis. We found six multilocus sequence types that were significantly associated with clinical manifestations in humans and five IGS haplotypes that were associated with the human Lyme borreliosis cases. While IGS could perform just as well as the housekeeping genes in the MLST scheme for predicting the infectivity of $B$. burgdorferi s.I., the advantage of MLST is that it can also capture the differential invasiveness of the various STs. 
In this thesis, I have identified the most important vertebrate hosts for maintenance of $B$. burgdorferi s.l. in enzootic cycles. I have also shown that their density is reflected in the prevalence of $B$. burgdorferi s.l. in questing ticks. The comparative study of questing ticks and Lyme borreliosis indicated that some of the Borrelia genospecies have similar prevalences in the two sources. The findings in my thesis indicate, thus, that there is a link between the density of suitable hosts for ticks and Borrelia spp., the density of infected ticks and the distribution of the B. burgdorferi s.l. genospecies in Lyme borreliosis. There are exceptions, however, that cannot be explained by this simple thread line. Such a situation is the perceived association of $B$. bavariensis with rodents that is not reflected by its extremely low prevalence in questing ticks. Furthermore, this low prevalence cannot explain the overrepresentation of B. bavariensis in Lyme borreliosis. As result of the study of pathogenicity of the various Borrelia genospecies and genotypes, I suggest the separate hazard assignment for the Borrelia genospecies; this, in combination with the exposure (prevalence in questing ticks) would allow for individual genospecies/genotypes risk assessment. The findings in this thesis stress the importance of both ecological and clinical studies for addressing the public health issue of Lyme borreliosis. 


\section{Acknowledgements}

It may be difficult to find the right words for thanking our peers and it might seem impersonal to align all the people who made a difference for your personal or professional life on a sheet of paper hidden somewhere at the end of a book. I hope, however, that most of the people mentioned here already know that I am grateful for our collaboration, for their support, for the fun we had together, or simply for being around. I hope that in these years I found at least once, the time to say it. If not...

First and foremost I thank my co-promotor and daily supervisor Hein Sprong for the trust in me you showed four years ago and for the opportunity you gave me by this research position. But there's more than that to be thankful for - it's also the freedom offered in doing my research and the networking opportunities you created for me, for the always positive feed-back I received on the manuscripts and ideas. I couldn't have had a better supervisor!

Willem Takken and Herbert Prins took the task of being my promotors and I thank them for that. In particular I thank Willem who always tried to keep me on track and on time and supported me with administrative issues to the very last stretch of this PhD. Thank you Willem!

Katsuhisa Takumi played an important part in this endeavour called PhD. Whenever I would present my results in a soft, descriptive manner, Katsuhisa would push me to think analytically, to support the conclusion with the adequate test, to dig some more into statistics and population genetics. Thank you Katsuhisa for all your support!

My fellow PhD students at WUR - Tim and Gilian - I couldn't have done this without your help. Starting from sharing samples and ideas and all the way to generating results and writing articles together you have proven wonderful colleagues, and I thank you for that.

My fellow PhD students at RIVM - Ellen, Katharina, Seta, Frits, Ankie, Eri, Jeroen, thanks to you I started my PhD programme with a week of parties (borrels, house-warming, etc) and that made my integration in the group and Dutch culture smoother. Thank you guys for all the fun we had together, from the RIVM Journal Club meetings, to the Carnaval parties!

For four years I was proud to be part of Tick research group and I enjoyed working with Manoj and Arnout. Arieke and Aleksandra, even if our work contacts were not very extensive I enjoyed it nevertheless. Arnout gets also 
special thanks for the moral support and the nice chats we had as kamergenoten.

The person who helped me most with the lab work is Kristel van Rooijen and even though she was around for little time she made a big difference. For your effort to speak English, for your patience with my not exactly organized manner of working, and for all the good work I owe you big thanks Kristel!

Trebuie să le mulţumesc părinţilor mei pentru sprijinul de care au dat dovadă, fiind întotdeauna înţelegători cu mine, chiar şi atunci când aceasta a însemnat plecarea mea la 2000 de km distanţă. După o mulţime de ani de studiu prin diverse şcoli şi universităţi sunt încă principii şi valori pe care nu le poţi învăţa decât de acasă. Mami şi Tati, pentru tot ceea ce mi-aţi oferit, şi mai ales pentru dragostea voastră, mulţumesc pare un cuvânt prea mic. Poate "MULJUMESC!" este mai bine.

My coming to The Netherlands meant new experiences, new people and changes, and not only in the professional life.

Daniela and Hen, my two expat friends that I made in The Netherlands, you were my reference points from the expat perspective. It was nice to have met you and I thank you for sharing your tips and advices stemming from your own experiences.

Ik heb in Nederland twee families gekregen, naast die van Roemenië: Familie Swart en Familie Pals. Dank jullie allemaal dat jullie mij in jullie midden gekregen hebben, voor de mooie vakanties en feestdagen die wij samen hebben doorgebracht, voor jullie advies, steun en zelfs voor jullie eigenwijsheid om alleen maar Nederlands tegen mij te praten.

Een Swart in het bijzonder wil ik bedanken voor het begrip en geduld voor mijn lange werkuren, frustraties, mopperen, grillen, nostalgieen etc., en voor het altijd opvrolijken, en het zien van de positieve kanten van de dingen en van mij. Dank je wel mijn lieve Arno! 


\section{Curriculum vitae}

Elena Claudia SWART-COIPAN is born on 09-02-1979 in Bucharest, Romania. After participation in the Biology Olympiads, and the graduation of the Chemistry-Biology profile of the "Eugen Lovinescu" high school, there was just one step to following a biologist career. After four years of study at the Faculty of Biology, University of Bucharest, and a Bachelor of Science in Microbiology, Claudia followed a Master of Science programme in Molecular biology and Genetics at the same university.

From 2001 she worked as Junior researcher at the National Institute for Microbiology and Immunology "Cantacuzino", Bucharest, in the Laboratory of Medical Entomology. The participation in an European FP6 project made Claudia consider to experience a change in the work environment. In 2012 she enrolled on a PhD track within a project financed by De Nederlandse Organisatie voor Wetenschappelijk Onderzoek (NWO) - "Shooting the messenger" under the supervision of Prof. Willem Takken at Wageningen University and Research and Dr. Hein Sprong at Het Rijksinstituut voor Volksgezondheid en Milieu (RIVM).

Since October 2016 Claudia works as microbial ecologist at Wageningen Plant Research within Wageningen University and Research. 


\section{Publications}

Coipan E.C., van Duijvendijk G., Krawczyk A.I., Hofmeester T.R., Swart A.N., Takumi K., Sprong H. Contribution of rodent species to maintenance of tick-borne pathogens in enzootic cycles. (submitted)

Hofmeester T.R., Jansen P.A., Wijnen H.J., Coipan E.C., Fonville M., Prins H.H.T., Sprong H., van Wieren S.E. Cascading effects of changes in vertebrate assemblages on rodent-transmitted tick-borne disease risk. (submitted)

Estrada-Peña A., Sprong H., Cabezas-Cruz A., de la Fuente J., Ramo A., Coipan E.C. Nested coevolutionary networks shape the ecological relationships of ticks, hosts and the Lyme disease bacteria Borrelia burgdorferi. Parasites and Vectors, 9:517.

Hofmeester T.R., Coipan E.C., van Wieren S.E., Prins H.H.T., Takken W., Sprong H. 2016. Few vertebrate species dominate the Borrelia burgdorferi s.l. life cycle. Environmental Research Letters, 11: 043001.

Ruyts S.C., Ampoorter E., Coipan E.C., Baeten L., Heylen D., Sprong H., Matthysen E., Verheyen K.. 2016. Diversifying forest communities may change Lyme disease risk: extra dimension to the dilution effect in Europe. Parasitology, 13: 1-10.

Coipan E.C., Jahfari S., Fonville M., Oei G.A., Spanjaard L., Takumi K., Hovius J.W., Sprong H.. 2016. Imbalanced presence of Borrelia burgdorferi s.I. multilocus sequence types in clinical manifestations of Lyme borreliosis. Infection Genetics and Evolution, 42: 66-76.

Kalmár Z., Sprong H., Mihalca A.D., Gherman C.M., Dumitrache M.O., Coipan E.C., Fonville M., Cozma V. 2016. Borrelia miyamotoi and Candidatus Neoehrlichia mikurensis in Ixodes ricinus Ticks, Romania. Emerging Infectious Diseases, 22 (3): 550-551.

Szekeres, S., Coipan, E.C., Rigó, K., Majoros, G., Jahfari, S., Sprong, H., Földvári, G. 2015. Ecoepidemiology of Borrelia miyamotoi and Lyme borreliosis spirochetes in a popular hunting and recreational forest area in Hungary. Parasites and Vectors, Jun 6; 8: 309

Szekeres, S., Coipan, E.C., Rigó, K., Majoros, G., Jahfari, S., Sprong, H., Földvári, G. 2015. Candidatus Neoehrlichia mikurensis and Anaplasma phagocytophilum in natural rodent and tick communities in Southern Hungary. Ticks and Tick Borne Diseases, Mar; 6 (2): 111-116.

Jahfari, S., Coipan E.C., Fonville, M., van Leeuwen, A.D., Hengeveld, P., Heylen, D., Heyman, P., van Maanen, C., Butler, C.M., Földvári, G., Szekeres, S., van Duijvendijk, G., Tack, W., Rijks, J.M., van der Giessen, J., Takken, W., van Wieren, S.E., Takumi, K., Sprong, H. 2014. Circulation of four Anaplasma phagocytophilum ecotypes in Europe. Parasites and Vectors, Aug 15; 7 : 365. doi: 10.1186/1756-3305-7-365.

Földvári G, Jahfari S, Rigó K, Jablonszky M, Szekeres S, Majoros G, Tóth M, Molnár V, Coipan E.C., Sprong H. 2014. Candidatus Neoehrlichia mikurensis and Anaplasma phagocytophilum in urban hedgehogs. Emerging Infectious Diseases, Mar; 20 (3): 496-498.

Coipan E.C., Jahfari, S., Fonville, M., Maassen, C.B., van der Giessen, J., Takken, W., Takumi, K., Sprong, H. 2013. Spatiotemporal dynamics of emerging pathogens in questing Ixodes ricinus. Frontiers in Cellular and Infection Microbiology, Jul 30;3:36

Coipan E.C., M. Fonville, E. Tijsse-Klasen, J.W. van der Giessen, W. Takken, H. Sprong, K. Takumi. 2013. Geodemographic analysis of Borrelia burgdorferi sensu lato using the 5S-23S rDNA spacer region. Infection, Genetics, and Evolution, Jul;17:216-22

Magdalena, L.M., E.C. Coipan, A.F. Vladimirescu, L. Savu, M. Costache, and L. Gavrilă. 2012. Downregulation of Drosophila melanogaster hsp22 gene expression in sites located near chemical plants. Genetics and Molecular Research, 11(1): 739-745.

Coipan E.C. \& Al.F. Vladimirescu. 2011. Ixodes ricinus ticks (Acari: Ixodidae) - vectors for Lyme disease spirochetes in Romania. Experimental and Applied Acarology, 54 (3): 293-300. 


\section{PE\&RC Training and Education Statement}

With the training and education activities listed below the PhD candidate has complied with the requirements set by the C.T. de Wit Graduate School for Production Ecology and Resource Conservation (PE\&RC) which comprises of a minimum total of 32 ECTS (= 22 weeks of activities)

\section{Review of literature (4.5 ECTS)}

- Few vertebrate species dominate the Borrelia burgdorferi s.I. life cycle

Writing of project proposal (4.5 ECTS)

- Transmission dynamics of Borrelia burgdorferi: a population genetics approach

Post-graduate courses (4.8 ECTS)

- Workshop on molecular evolution; Evomics.org, Český Krumlov, Czech Republic (2013)

- Course on next generation sequencing; Eurnegvec.org, Bruxelles, Belgium (2015)

Invited review of (unpublished) journal manuscript (2 ECTS)

- Parasites and vectors: tick-borne pathogens (2013)

- Infection, Genetics and Evolution: genomics (2014)

Deficiency, refresh, brush-up courses (2.1 ECTS)

- Basic statistics; PE\&RC (2012)

- Introduction to R for statistical analysis; PE\&RC (2013)

Competence strengthening / skills courses (6 ECTS)

- Techniques for writing and presenting a scientific paper; In'to Languages (2013)

- Presentation skills; In'to Languages (2013)

- Scientific writing; In'to Languages (2013)

- Writing grant proposals; In'to Languages (2016)

PE\&RC Annual meetings, seminars and the PE\&RC weekend (1.5 ECTS)

- PE\&RC Weekend last year (2015)

- PE\&\&RC Day: one's waste....another's treasure? (2015)

- NERN Symposium: current themes in ecology (2015)

Discussion groups / local seminars / other scientific meetings (4.8 ECTS)

- Wageningen evolution and ecology seminars (2012)

- RIVM Journal club (2012-2014)

International symposia, workshops and conferences (10.4 ECTS)

- E-SOVE; Montpellier, France (2012)

- EurNegVec COST International meeting; Cluj-Napoca, Romania (2014)

- MEEGID International conference; Bangkok, Thailand (2014)

- EurNegVec COST International meeting; Izmir, Turkey (2015)

- MEEGID International conference; Antwerpen, Belgium (2016) 
